\title{
An Analysis of Treatment Patterns, Receipt of Guideline- Concordant Care, and Survival Outcomes among Elderly Women with Non-Metastatic Breast Cancer Using the SEER-Medicare Linked Dataset
}

Traci LeMasters

Follow this and additional works at: https://researchrepository.wvu.edu/etd

\section{Recommended Citation}

LeMasters, Traci, "An Analysis of Treatment Patterns, Receipt of Guideline-Concordant Care, and Survival Outcomes among Elderly Women with Non-Metastatic Breast Cancer Using the SEER-Medicare Linked Dataset" (2015). Graduate Theses, Dissertations, and Problem Reports. 6057.

https://researchrepository.wvu.edu/etd/6057

This Dissertation is protected by copyright and/or related rights. It has been brought to you by the The Research Repository @ WVU with permission from the rights-holder(s). You are free to use this Dissertation in any way that is permitted by the copyright and related rights legislation that applies to your use. For other uses you must obtain permission from the rights-holder(s) directly, unless additional rights are indicated by a Creative Commons license in the record and/ or on the work itself. This Dissertation has been accepted for inclusion in WVU Graduate Theses, Dissertations, and Problem Reports collection by an authorized administrator of The Research Repository @ WVU.

For more information, please contact researchrepository@mail.wvu.edu. 
An Analysis of Treatment Patterns, Receipt of Guideline-Concordant Care, and Survival Outcomes among Elderly Women with Non-Metastatic Breast Cancer Using the SEERMedicare Linked Dataset

$$
\text { Traci LeMasters }{ }^{1}
$$

Dissertation submitted to the School of Pharmacy at West Virginia University

in partial fulfillment of the requirements for the degree of

Doctor of Philosophy

in

Health Outcomes Research

S. Suresh Madhavan, M.B.A, Ph.D. Chair ${ }^{1}$

Usha Sambamoorthi Ph.D. ${ }^{1}$

Kimberly M Kelly Ph.D. ${ }^{1,2}$

Dustin Long Ph.D. ${ }^{3}$

Hannah Hazard M.D. ${ }^{4}$

1 Department of Pharmaceutical Systems and Policy, School of Pharmacy, West Virginia University

2 Mary Babb Randolph Cancer Center, West Virginia University

${ }^{3}$ Department of Biostatistics, School of Public Health, West Virginia University

${ }^{4}$ Department of Medical Education, School of Medicine, West Virginia University

Morgantown, West Virginia

2015

Keywords: Breast Cancer, Treatment Patterns, Guideline-Concordant Care, Survival, Elderly, Loco-Regional Treatment, Radiation Therapy, Chemotherapy, Undertreatment 


\section{ABSTRACT \\ An Analysis of Treatment Patterns, Receipt of Guideline-Concordant Care, and Survival Outcomes among Elderly Women with Non-Metastatic Breast Cancer Using the SEER- Medicare Linked Dataset}

\section{Traci LeMasters}

Breast cancer (BC) is the 2nd most commonly diagnosed type of cancer in the United States (US) and the 1 st among women, with $57 \%$ of incident cases in those age $\geq 60$ years. Relative to other cancers, BC has high survival rates, with a 89\% 5-year overall survival rate. High survival rates are due to improvements in disease understanding, treatment, and earlier stage at diagnosis from increased routine $\mathrm{BC}$ screening. Yet, disparities in treatment and survival outcomes persist. Epidemiologic studies suggest that elderly women experience disparities uniquely associated with increasing age and comorbidity, in addition to those associated with socio-demographic characteristics, access to oncology care resources, and clinical prognostic factors. This sequence of retrospective database studies sought to characterize and examine associations with initial locoregional treatment for stage I and II BC, receipt of guideline-concordant care (GCC) and individual tests and treatments for stage I-III BC, and overall 5-year survival among using the first two study cohorts and a third, more broadly inclusive cohort of elderly women with stage I-III BC. Cohorts of women age $\geq 66$ years diagnosed in 2003-2009 were selected from the Surveillance, Epidemiology, and End Results-Medicare (SEER-Medicare) linked dataset. Regarding the 1st study, 55\% of women had breast-conserving surgery (BCS) plus radiation therapy (RT), 23\% has mastectomy, and 22\% had BCS without RT as their initial loco-regional treatment. Compared to women who received BCS plus RT, those who were older, of greater comorbidity, later stage, or non-white race were more likely to have had mastectomy or BCS without RT. Women who were less likely to have had mastectomy or BCS without RT were those treated by an oncology surgeon or both an oncology and general surgeon vs. a general surgeon only, from areas of less education, lower income, or lived in metro areas. Regarding the 2nd study, only 34\% received GCC, $61 \%$ had RT, and $25 \%$ had chemotherapy but, most women had their hormone receptor (HR) statuses and lymph nodes tested. Women who were older, of greater comorbidity, stage II vs. I, lymph node negative, or non-white race were less likely to receive GCC, while those who were HR negative or treated by an oncology surgeon or both an oncology and general surgeon, vs. a general surgeon only were more likely to receive GCC. Regarding the 3rd study, overall 5-year survival ranged from 82\%-88\% among the three cohorts. The risk of death was greater for women who were older, of greater comorbidity, diagnosed at a later stage, HR negative, treated by mastectomy, BCS without RT, did not received GCC, RT, or chemotherapy, but was lower for women treated by an oncology surgeon or both an oncology and general surgeon vs. a general surgeon only. Despite recommended treatment guidelines, increasing age and comorbidity are strongly associated with less aggressive BC among elderly women. Older women with BC should receive treatment according to guidelines as it would be otherwise given to younger women, health permitting. While the increased risk of death associated with increasing age is inevitable, targeting health behaviors to decrease comorbidity and continued routine BC screening for earlier stage at diagnosis may go a long way to improve survival. 


\section{ACKNOWLEDGEMENTS}

I would like to thank my major advisor, Dr. Suresh Madhavan for his very wise and patient guidance over the years. I would not have reached this milestone in my life without his vision and continued encouragement. His mentorship has been invaluable. I would also like to thank Dr. Usha Sambamoorthi for her guidance and time she has spent with me and put into my research over the years. I would have the level of experience and skill with database analysis or learned how to write concisely for manuscript publication without her mentorship. I would also like to thank the remaining members of my dissertation committee that include Dr. Kimberly Kelly, Dr. Dustin Long, and Dr. Hannah Hazard. They have all graciously committed their time and unique expertise, helping to ensure the quality of my work.

Throughout the years all the faculty, staff, and fellow students in the Department of Pharmaceutical Systems and Policy have supported me personally and professionally. It is truly one of the best departments of be a member of at this university. I am grateful to have known and been a part of the PSP family over the years.

Last, but not least, I would like to thank my husband and family for continued and support and encouragement during this long-term endeavor. The journey to a Ph.D. is not swift or smooth. It is not a destination that would be reached if the road were to be traveled alone. It is for this reason that I owe much gratitude to all of these people. 


\section{TABLE OF CONTENTS}

TITLE PAGE...................................................................................................................................... i

ABSTRACT ............................................................................................................................................... ii

ACKNOWLEDGEMENTS............................................................................................................. iii

TABLE OF CONTENTS................................................................................................. iv

CHAPTER 1: OVERALL INTRODUCTION...................................................................... 1

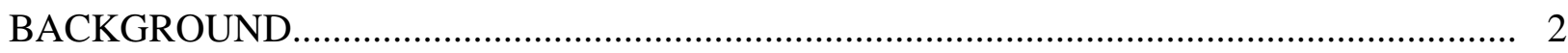

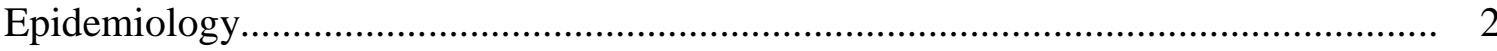

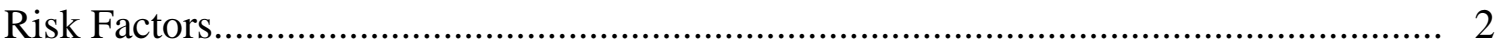

Types of Breast Cancer................................................................................................... 4

Breast Cancer Screening............................................................................................. 4

Diagnosis \& Characterization................................................................................. 5

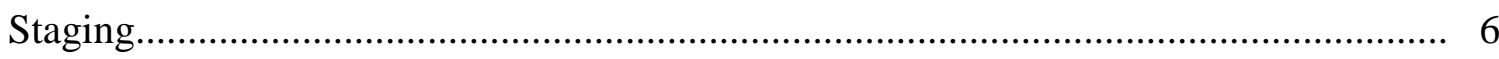

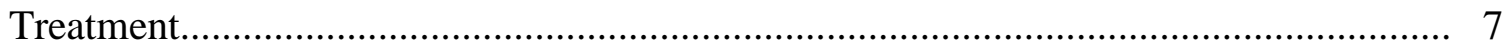

Stage 0 (LCIS) ..................................................................................... 8

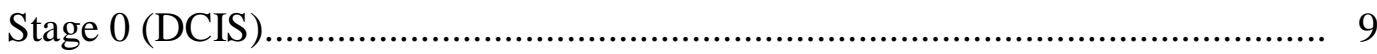

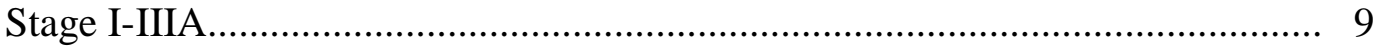

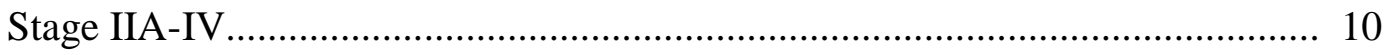

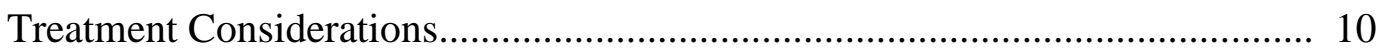

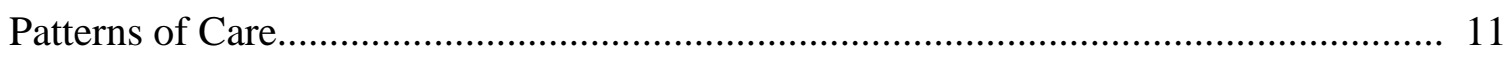

Survival \& Recurrence.......................................................................................... 13

STATEMENT OF THE PROBLEM........................................................................... 14

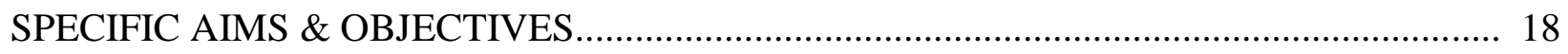

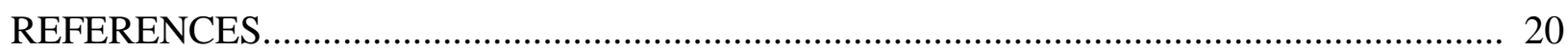

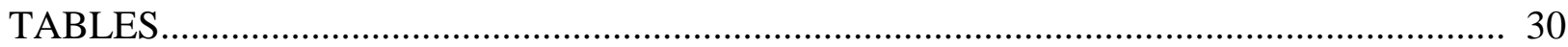


Table 1: Definition of "T", "N", and "M" Categories of the TNM Staging System....... 30

Table 2: TNM Staging System for Breast Cancer.............................................. 30

Table 3: Breast Cancer Survival, 2001-2007........................................................ 31

Table 4: Breast Cancer Mortality by Age, 2005-2009.......................................... 31

Table 5: Breast Cancer Survival by Race/Ethnicity, 2001-2007.............................. 31

CHAPTER 2: MANUSCRIPT \# 1.......................................................................................... 32

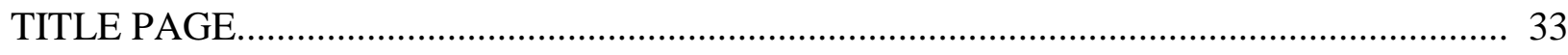

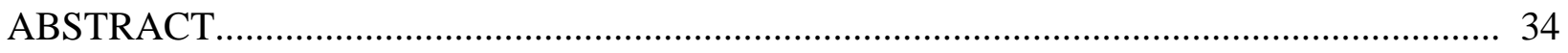

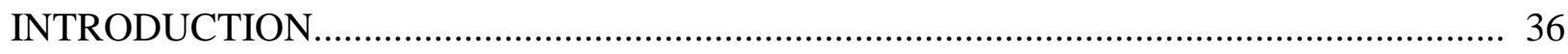

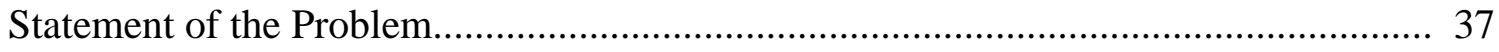

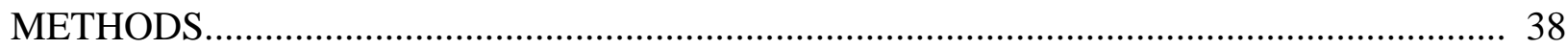

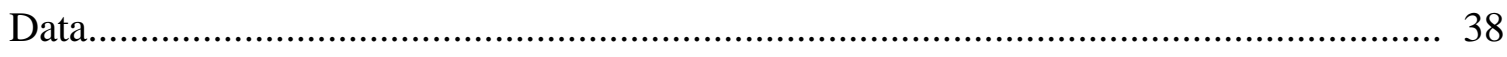

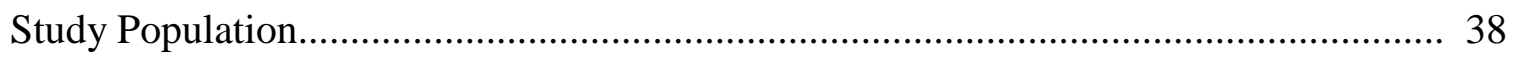

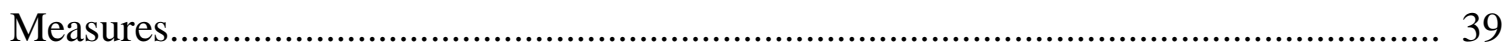

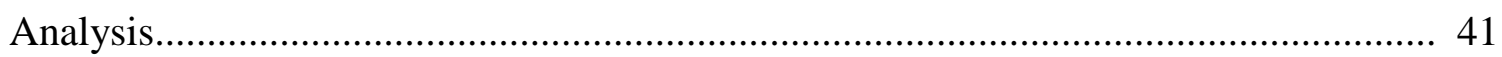

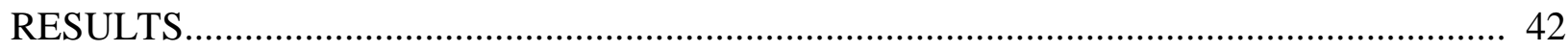

Study Population Characteristics............................................................... 42

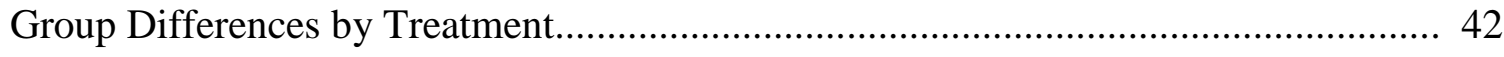

Associations with Type of Treatment.............................................................. 44

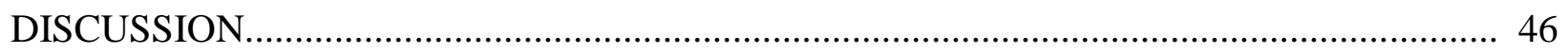

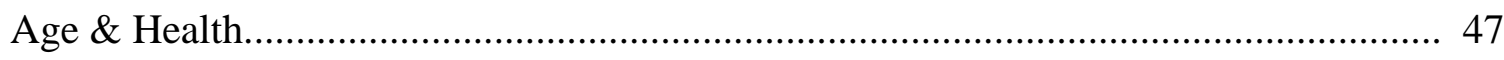

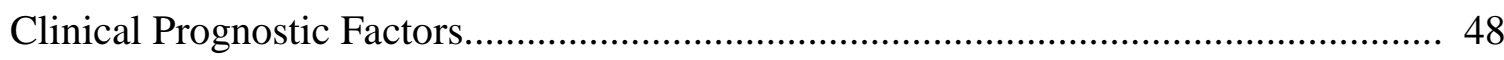

Oncology Care Resources.............................................................................. 49

Socio-Demographic Characteristics............................................................. 50

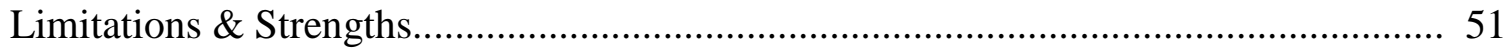




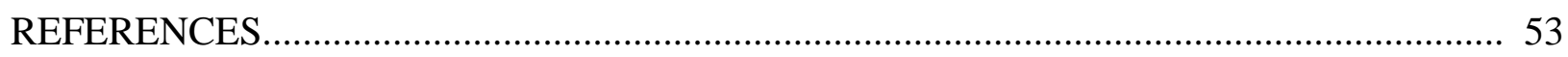

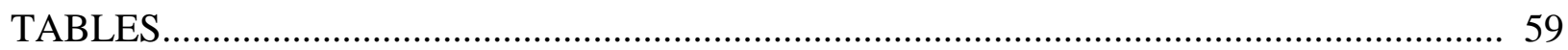

Table 1: Characteristics of Elderly Women with Early-Stage Breast Cancer................ 59

Table 2: Comparison of Initial Loco-Regional Treatment Among Elderly Women with Early-Stage Breast Cancer............................................................ 61

Table 3: Associations with Initial Loco-Regional Treatment Among Elderly Women with Early-Stage Breast Cancer......................................................... 63

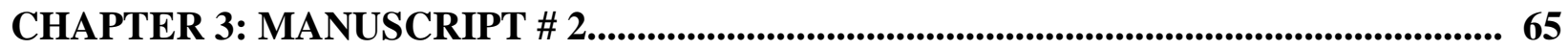

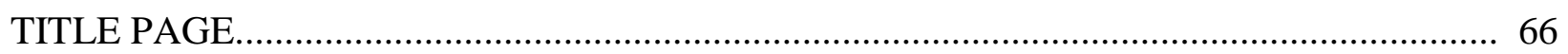

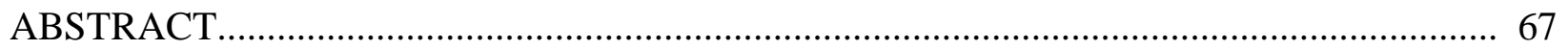

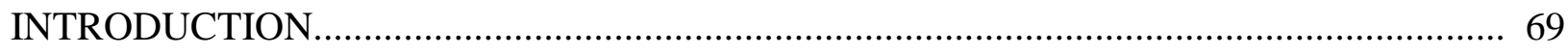

Statement of the Problem.................................................................................. 71

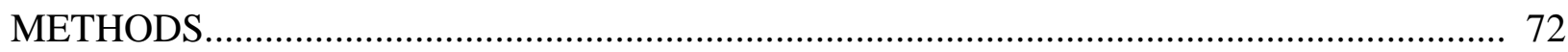

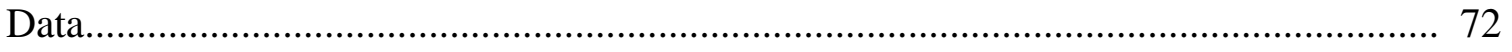

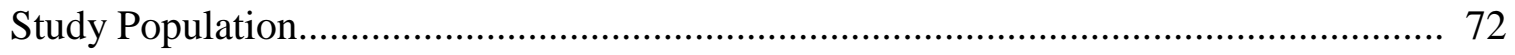

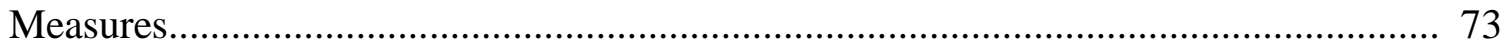

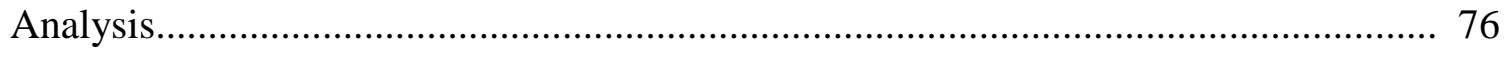

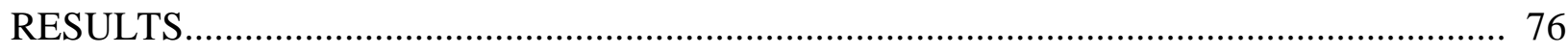

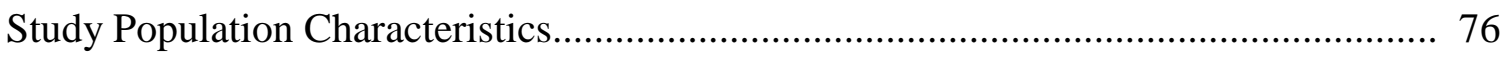

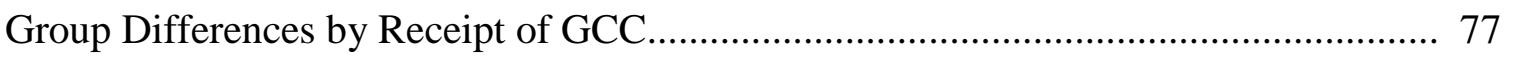

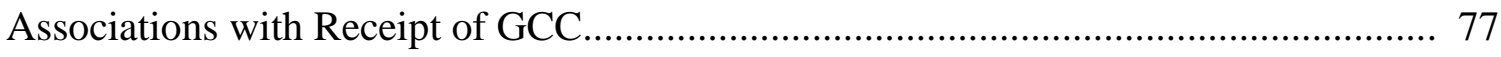

Associations with Tests \& Treatments among Women Age $\geq 70$ vs. Age 66-69 Years. 78

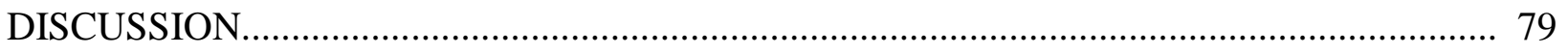

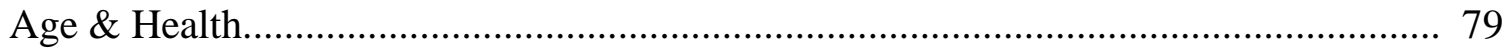




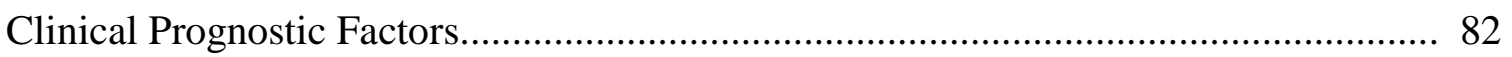

Oncology Care Resources...................................................................................... 82

Socio-Demographic Characteristics........................................................................... 83

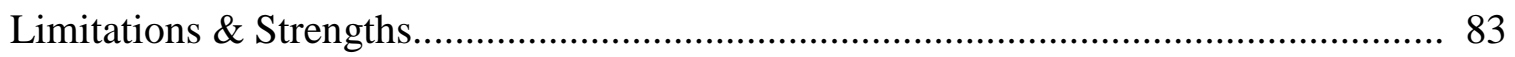

SIGNIFICANCE \& CONCLUSIONS............................................................................ 85

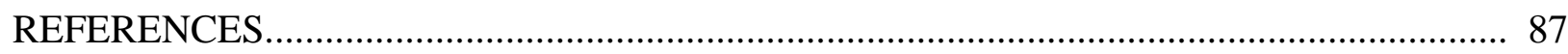

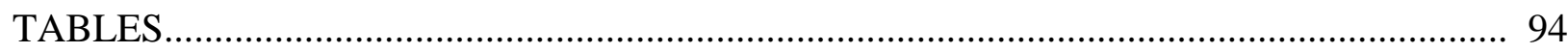

Table 1: Characteristics of Elderly Women with Stages I, II, and III Breast Cancer...... 94

Table 2: Comparison of Guideline-Concordant Care among Elderly Women with

Stages I, II, and III Breast Cancer.................................................................... 97

Table 3: Associations with Guideline-Concordant Care among Elderly Women with

Stages I, II, and III Breast Cancer................................................................. 100

Table 4: Associations with Tests \& Treatments among Elderly Women with Stages

I, II, and III Breast Cancer......................................................................... 102

APPENDICES.

Appendix 1: Claims Codes Used for Identifying Types of Treatment........................... 103

Appendix 2: Guideline-Concordant Care Treatment Options by Tumor Size \& Lymph

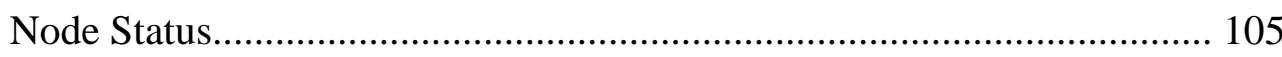

CHAPTER 4: MANUSCRIPT \# 3................................................................................... 106

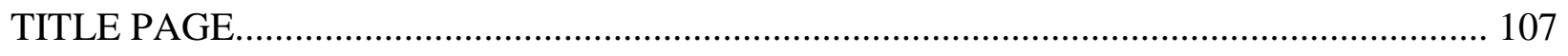

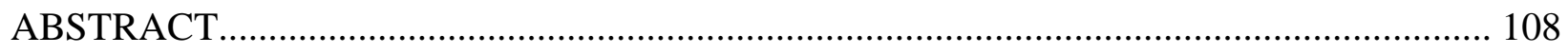

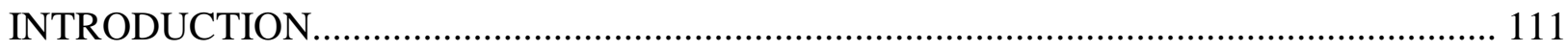

Statement of the Problem............................................................................................. 114

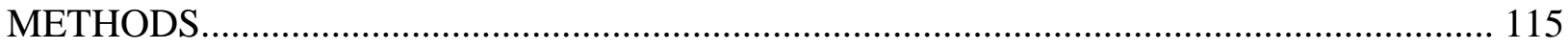

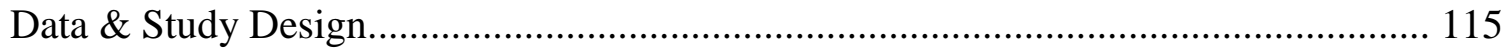




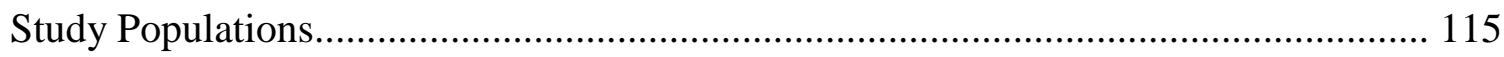

Cohort 1: All Women with Stage I, II, and III Breast Cancer............................... 115

Cohort 2: Local Treatment among Women with Stage I \& II Breast Cancer..... 116 Cohort 3: Guideline-Concordant Care among Women with Stage I, II, \& III

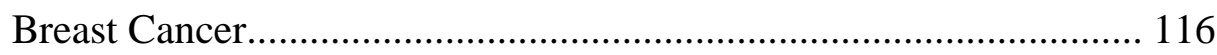

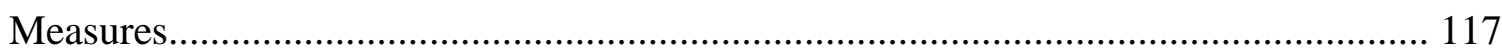

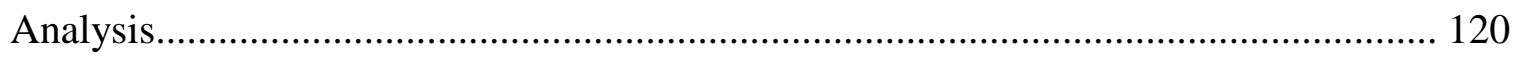

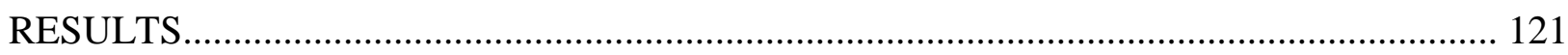

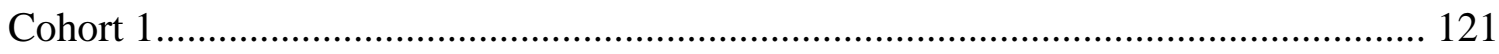

Cohort Characteristics.................................................................................. 121

Group Differences in 5-Year Survival............................................................ 121

Risk of Death within 5 Years of Diagnosis....................................................... 123

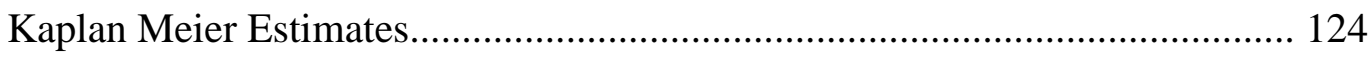

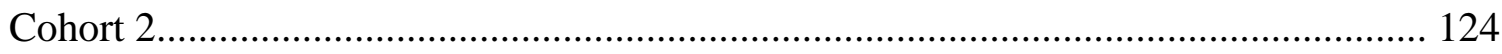

Cohort Characteristics................................................................................ 124

Group Differences in 5-Year Survival............................................................ 125

Risk of Death within 5 Years of Diagnosis..................................................... 126

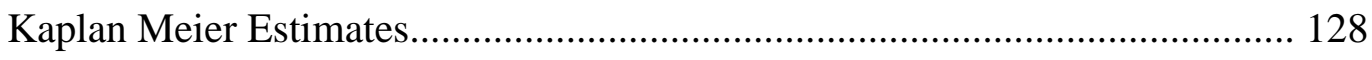

Cohort 3

Cohort Characteristics.................................................................................. 128

Group Differences in 5-Year Survival............................................................ 129

Risk of Death within 5 Years of Diagnosis....................................................... 131

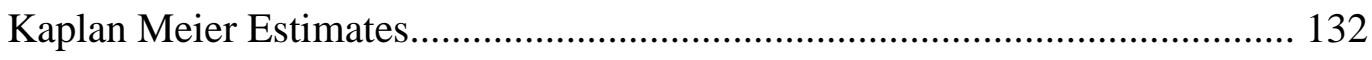

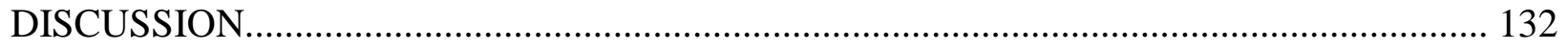

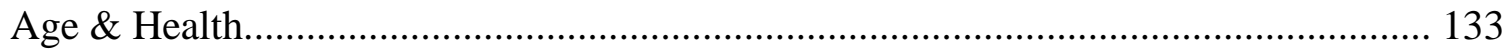


Clinical Prognostic Factors....................................................................................... 134

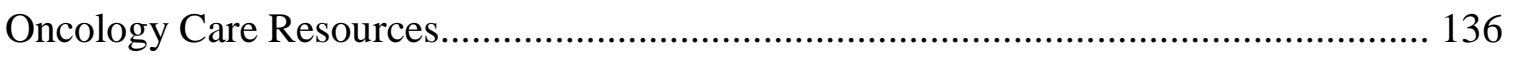

Socio-Demographic Characteristics.............................................................................. 137

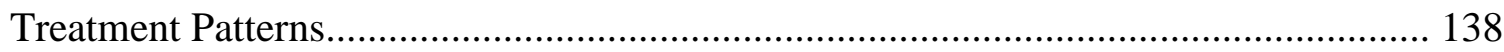

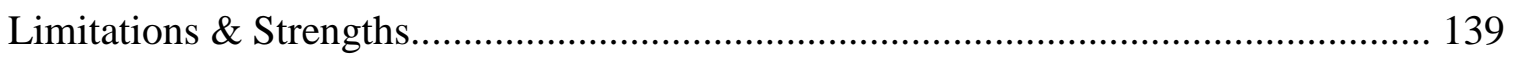

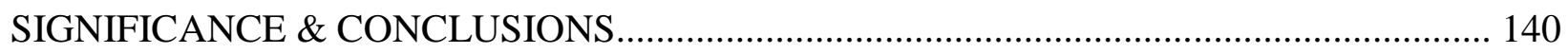

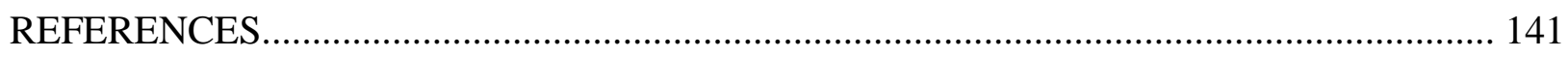

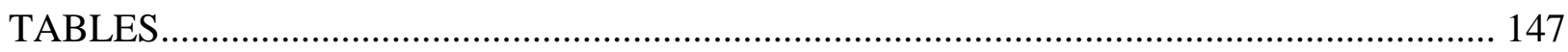

Table 1: Characteristics of Elderly Women with Invasive Non-Metastatic Breast

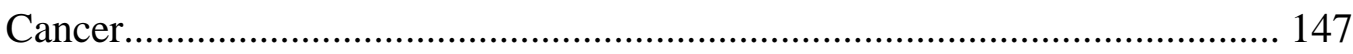

Table 2: Comparison of Characteristics Between Elderly Women Alive and Dead 5

Years After Invasive Non-Metastatic Breast Cancer Diagnosis........................ 150

Table 3: Hazard of Death 5 Years After Diagnosis of Invasive Non-Metastatic Breast

Cancer in Elderly Women ......................................................................... 155

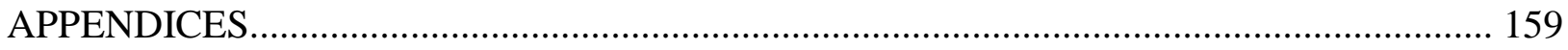

Appendix 1: Claims Codes Used for Identifying Types of Treatment.......................... 159

Appendix 2: Guideline-Concordant Care Treatment Options by Tumor Size \& Lymph

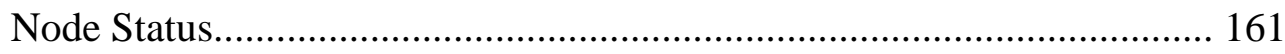

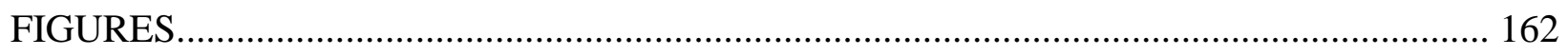

Figure 1: Survival Curve for Cohort 1..................................................................... 162

Figure 2: Survival Curve by Stage for Cohort 1........................................................ 163

Figure 3: Survival Curve for Cohort 2................................................................ 164

Figure 4: Survival Curve by Treatment for Cohort 2.................................................. 165

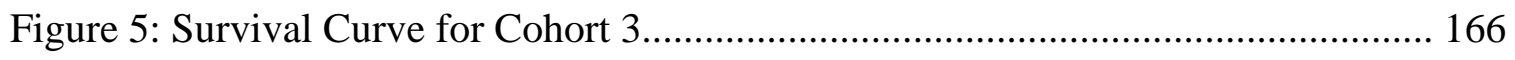

Figure 6: Survival Curve by GCC for Cohort 3........................................................... 167 


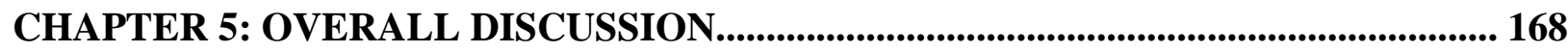

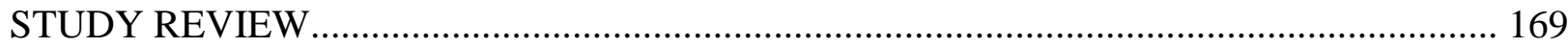

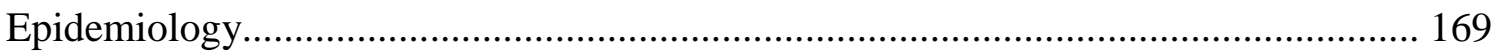

Disparities in Treatment \& Survival...................................................................... 169

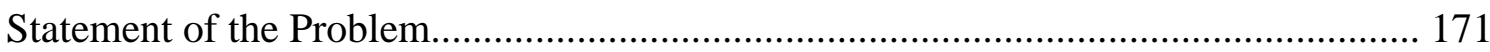

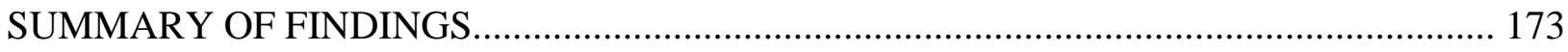

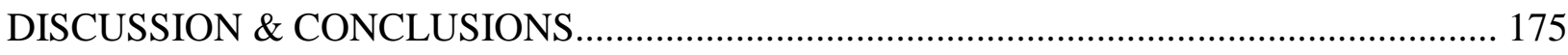

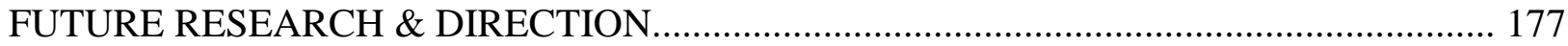

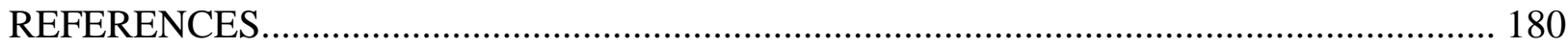


CHAPTER 1 


\section{CHAPTER 1}

\section{Background}

\section{Epidemiology}

Female breast cancer is the second most prevalent type of cancer diagnosed in the United States (US) population and the first among women. ${ }^{1}$ It is estimated that in $2014,232,670$ incident diagnoses and 40,000 deaths will be attributed to breast cancer. It is estimated that about 1/8 women will develop breast cancer in their lifetime and as of January $1^{\text {st }}, 2012$, over 2.9 million women with a history of breast cancer were living in the US. ${ }^{2}$ The average age at diagnosis is 61 years. About $20 \%$ of breast cancer cases occur in women age $\leq 50$ years, $40 \%$ among women age $50-64$ years, and $40 \%$ among women age $\geq 65$ years. ${ }^{3}$ Although breast cancer can occur in men, it only accounts for about $1 \%$ of incident cases. ${ }^{4}$

\section{$\underline{\text { Risk Factors }}$}

Non-modifiable risk factors include gender, age, genetic mutations, race, family history of breast cancer, personal history of breast cancer, history of DCIS or LCIS, age at menarche, age at menopause, and radiation to the chest. Female gender, age $\geq 55$ years, and genetic mutations increase the risk for breast cancer. Non-Hispanic White women have slightly higher rates of breast cancer overall, but in women age $<45$ years, rates are higher among non-Hispanic Blacks. Although $85 \%$ of women who develop breast cancer do not have an immediate family history of the disease (mother, sister, or daughter), women with an immediate family history are almost twice as likely to develop breast cancer. Women who begin their menstrual cycle at age < 12 years and experience menopause at age $>55$ years are at increased risk of developing breast cancer due to prolonged exposure to the female hormones estrogen and progesterone, which can stimulate malignant cell growth in breast tissue. Radiation to chest area, for treatment of an earlier cancer, increases a woman's chance of breast cancer if radiation was received earlier in 
life, especially during the teenage years of breast development, but does not appear to increase breast cancer risk if radiation treatment was received after the age of 40 years. ${ }^{2,5,6}$ About $5 \%$ $10 \%$ of incident cases of breast cancer are attributable to inheritable genetic mutations, with about $15 \%-20 \%$ of those mutations to the $B R C A 1$ and $B R C A 2$ genes. While genetic mutations are present in less than $1 \%$ of the population, they are more common among women of certain ethnic groups, particularly those of Ashkenazi (Eastern European) Jewish descent. Genetic testing for mutations to several genes that increase the risk of breast cancer are available, but interpretation of results and subsequent treatment decisions remains challenging. The United States Preventive Services Task Force (USPSTF) recommends that women with a very strong family history of breast cancer be tested for $B R C A$ mutations, while the American Cancer Society (ACS) and American Society for Clinical Oncology (ASCO) recommend that any women wanting genetic testing first meet with a genetic counselor to assess individual level of risk for mutations, and the benefits and harms of testing. ${ }^{4}$

Modifiable risk factors include age at first childbirth, history of breast feeding, use of oral contraception, use of hormone replacement therapy, alcohol consumption, weight, and exercise. Women who have not had children, or had children after the age of 30 years, are at a slightly higher risk of breast cancer due to prolonged estrogen and progesterone exposure. Similarly, use of oral contraceptives or combination estrogen/progesterone hormone replacement therapy after menopause increases breast cancer risk. Also, since breast feeding lowers a woman's total number of menstrual cycles, women who do not breast feed are at an increased risk for breast cancer. Elevated risk for breast cancer is also associated with a lack of exercise and being overweight or obese. Although the relationship between weight and breast cancer is not well understood, it is thought that women with higher percentages of body fat, particularly in the 
abdomen, have higher levels of estrogen in their bodies. Finally, women who consume $2-5$ alcoholic drinks a day are about 1.5 times more likely to develop breast cancer, compared to women who do not drink alcohol. ${ }^{2,5}$

\section{$\underline{\text { Types of Breast Cancer }}$}

Female breast cancer, from here on referred to as "breast cancer", can be classified into two general categories, non-invasive and invasive. Non-invasive breast cancers, called "in situ" are confined to either the ductal (ductal carcinoma in situ or DCIS) or lobular (lobular carcinoma in situ or LCIS) regions of the breast tissue and are benign masses. Ductal carcinoma in situ accounts for the majority ( $83 \%$ ) of non-invasive breast cancer diagnoses. Together, DCIS and LCIS comprise less than $11 \%$ of all breast cancer cases, but may increase the risk for developing invasive breast cancer. The majority of breast cancer cases are invasive and can spread to areas outside the breast tissue. Inflammatory breast cancer (IBC) is a rare form of invasive breast cancer and accounts for $1 \%$ - $3 \%$ of all breast cancer cases. It is not characterized by any single lump or tumor, making it hard to detect at an early stage. ${ }^{2,4}$

\section{Breast Cancer Screening}

Methods of breast cancer screening include mammography, magnetic resonance imaging (MRI), ultrasound, and clinical breast exam (CBE). However, mammography is used at the primary method to detect breast cancer. Recommended guidelines for the use of mammography screening vary across agencies and remain controversial.

The American Cancer Society (ACS) and the National Comprehensive Cancer Network (NCCN) recommend annual mammograms for women age $\geq 40$ years. ${ }^{2,7}$ The United States Preventive Services Task Force (USPSTF) had previously recommended biennial screening mammography in women age $\geq 40$ years. However, in 2009 the USPSTF released an updated 
statement that recommended against routine screening mammography in women $40-49$ years of age, biennial screening mammography for women 50 - 74 years of age, and rescinded any recommendation for screening mammography among women age $\geq 75$ years. Changes to recommended screening mammography guidelines were due to an internal USPSTF review of evidence concluding that mammography resulted in only a small net benefit among women 40 49 and benefits were inconclusive evidence among women age $\geq 75$ years. ${ }^{8}$

The sensitivity and specificity of mammography varies by study and patient characteristics. The overall sensitivity of mammography ranges from $67.8 \%-84.1 \% .{ }^{9-12}$ Mammography has been found to be less sensitive in younger women with denser breast tissue $(33 \%-50 \%)$, compared to more fatty breast tissue $(75 \%-100 \%){ }^{2,4,9}$ The overall specificity of mammography ranges from $75 \%-92 \%$, but is also lower in younger women with more dense breast tissue. $^{9,11,12}$ Additionally, longer intervals between mammography have been associated with increased sensitivity and decreased specificity. ${ }^{13}$

Reasons for false-positive mammography results include dense breast tissue, reading by a less experienced radiologist, time since last screening, and availability of prior mammography film for comparison. Additionally, benign breast abnormalities such as micro calcifications, fibro adenomas, and cysts can make it difficult to distinguish between benign and cancerous lesions.

\section{Diagnosis \& Characterization}

Women with screening mammography results found to be normal, require no additional testing and should continue routine screening. However, if a suspicious image cannot be determined as benign or malignant, then a diagnostic mammogram should be performed, with or without a breast ultrasound. Although, breast ultrasound is a complementary method to 
mammography, it does not detect the majority of micro calcifications. Mammography results determined to be positive for breast cancer will require additional imaging and tests that may include diagnostic mammography, breast ultrasound, MRI, and breast biopsy. Methods for a breast biopsy include Fine Needle Aspiration Biopsy (FNA), Core Needle Biopsy (CNB), and Excisional Biopsy.

Additional characteristics used to classify the type of cancer are the presence or absence of certain hormone receptors. If receptors for estrogen or progesterone are present in the tumor, they can also stimulate the growth of the tumor. Similarly, when the growth promoting protein HER-2/neu is present, tumors will grow and spread more rapidly. Hormone receptor assay tests identify the presence or absence of these hormone receptors. If a hormone receptor is present the breast cancer is further classified as estrogen-receptor positive (ER-positive), progesteronereceptor positive (PR-positive), and/or HER-2 positive. These hormone classifications, in conjunction with staging, determine the appropriate course of treatment. ${ }^{14}$

\section{Staging}

Once a case of breast cancer has been diagnosed, the stage of cancer development is used to characterize the severity and/or extent that the cancer has spread. Two commonly used systems of staging are the American Joint Committee on Cancer (AJCC) TNM (See Tables 1 \& 2) and the Surveillance Epidemiology End Results (SEER) Summary Stage systems. ${ }^{14,15}$ The SEER Summary Stage system for breast cancer is a more general system of staging breast cancer. Under this system, stages are categorized into three groups: local-stage, regional-stage, and distant-stage. In the local-stage, the cancer is confined to the breast tissue. In the regionalstage, the breast cancer has spread to surrounding tissue and lymph nodes. In the distant-stage, the breast cancer has metastasized to distant organs. ${ }^{4}$ 


\section{$\underline{\text { Treatment }}$}

Local treatment focuses only on the area of the breast where the cancer is confined to, while systematic treatment is given throughout the entire body to treat cancer that has spread beyond the breast. Local treatment includes surgery and radiation therapy. The two primary surgeries used to remove breast cancer are breast conserving surgery (BCS), i.e. lumpectomy, and mastectomy. Many women may also undergo a lymphadenectomy to remove affected lymph nodes. BCS removes the cancerous tumor and a small rim of healthy tissue surrounding the tumor for earlier stage breast cancer. Surgical mastectomy removes the entire breast for later stage diagnoses, or upon patient request. Two forms of lymphadenectomy are used to remove affected lymph nodes. A sentinel lymph node biopsy examines lymph nodes when there is no evidence that the cancer has spread to the nodes. A radioactive dye is injected into the breast, and as the dye leaves the breast and travels through the lymph system, it leaves a path identifying the first node nearest the breast. This node is removed and tested for cancer. If the test is negative, no further node removal is necessary, otherwise more nodes will be removed. The number of affected nodes influences the course of treatments. An axillary lymph node dissection is used to remove lymph nodes under the armpit when there is sufficient evidence of malignancy. Radiation therapy, another form of local treatment, directs a beam of high-energy rays to the chest and breast area. External beam radiation therapy (EBRT) is most often used after BCS 5 days of week for 6 to 7 weeks to destroy any remaining cancerous cells. Radiation therapy is recommended after BCS and after mastectomy if the tumor is $>5 \mathrm{~cm}$. Radiation is also used to treat the area where affected lymph nodes were removed.

Systematic treatment can be chemotherapy, hormone therapy, and targeted therapy. Chemotherapy is a single drug or a mix of drugs used to kill cancerous cells that can be 
administered intravenously or orally with a pill. Chemotherapy is used if cancerous cells have spread throughout the body in early stage breast cancers, or when the cancer has metastasized to other parts of the body in later stages, to control the tumor growths. Chemotherapy can also be used as neoadjuvant treatment to shrink tumors $>5 \mathrm{~cm}$ before surgery to remove the tumor. Treatment is given in cycles of 14,21 , or 28 days, with days of rest in between over a period of 3 to 6 months.

Hormone therapy treats tumors that test positive for estrogen and/or progesterone receptors. Estrogen and progesterone are hormones that promote the growth of normal breast tissue in women, but can also promote the growth of cancerous cells when present in breast tumors. Hormone therapies work by either blocking the receptors from receiving the hormones or by reducing the body's hormone production. Adjuvant hormone therapy is typically prescribed for 5 years to women with early stage breast cancers after primary treatment to prevent recurrence. Hormone therapy may be given as the first line of treatment for advanced staged breast cancer.

Targeted therapy is form of systematic treatment that targets only cancerous cells. When the protein for the Human Epidermal Growth Factor Receptor 2 is found in breast tumors, the breast cancer is classified as HER-2/neu positive. Trastuzumab is a targeted intravenous drug therapy that may be given as a neo-adjuvant to shrink the tumor before surgery, but it is most often given as an adjuvant treatment for 1 year. It is commonly started when chemotherapy is initiated. $^{14}$

Stage 0 (LCIS)

LCIS is usually not treated with any surgery other than a surgical biopsy. Depending on individual preference and level of risk, a woman may choose to take hormone therapy for 5 years 
to reduce the risk of recurrence. Some women may also choose to have a prophylactic mastectomy, but this option should only be chosen for high risk women who first undergo genetic counseling. ${ }^{14}$

Stage 0 (DCIS)

DCIS is most often treated with BCS. If there is only 1 tumor and the margin is noncancerous, radiation therapy may not be necessary, depending on the patient's risk for recurrence. If the rim of tissue removed around the tumor is positive for cancerous cells, the patient may require another excision or a mastectomy if the cancer cannot be completely removed or continues to return. BCS should be followed up with radiation therapy, but if a mastectomy is performed, then radiation therapy is not necessary. Some patients may choose to have a mastectomy when it is not necessary for preventive reasons. However, studies have shown that women who receive BCS with radiation have the same survival rates as women treated with mastectomy. If primary treatment includes BCS, adjuvant hormone therapy should be given to prevent recurrence if the tumor is estrogen or progesterone receptor positive. ${ }^{14}$ Stages I - IIIA

Women diagnosed with breast cancer Stage IA, IB, IIA, IIB, and some cases of Stage IIIA will have the option for BCS. Patients with tumors $>2 \mathrm{~cm}$, and who desire to have BCS vs. mastectomy should consider neo-adjuvant chemotherapy to shrink the tumor. BCS should be followed by radiation therapy. Patients with tumors $>2 \mathrm{~cm}$ that do not shrink with first or second rounds of chemotherapy will require a mastectomy. Depending on the degree of nodal involvement, patients will have any necessary lymph node removal at the time of tumor removal or shortly after. Adjuvant chemotherapy may be given for patients with tumors $>1 \mathrm{~cm}$, have 2 or more cancerous lymph nodes, or at high risk of recurrence. If the patient's tumor was found to be 
HER-2/neu positive, they will start targeted therapy at the time of diagnosis. If the patient's tumor is estrogen or progesterone receptor positive, they will begin adjuvant hormone therapy after radiation. ${ }^{14}$

Stages IIIA - IV

Some patients diagnosed with Stage IIIA breast cancer may still have the option of BCS if the tumor can be shrunk with neo-adjuvant chemotherapy. However, most patients with Stage IIIA and beyond will undergo mastectomy. Patients in these stages will most likely also require radiation therapy after surgery, regardless of which type of surgery they receive, due to the advanced stage of the disease and lymph node involvement. Moreover, patients in theses stages will most likely undergo neo-adjuvant and adjuvant chemotherapy. Targeted therapy and adjuvant hormone therapy will also be given if tumors are receptor positive for either type of hormones. Patients with Stage IV breast cancer that has metastasized to other areas of their body may have inoperable tumors, but may still elect for mastectomy to relieve pain. Combinations of radiation therapy, chemotherapy, targeted therapy, and hormone therapy can be used to shrink and slow cancer growth and spread in order to prolong life. ${ }^{14}$

\section{Treatment Considerations}

While these are recommended guidelines for breast cancer treatment put forth by the NCCN, treatment should and may vary according to individual patient characteristics such as age, health, and menopausal status. Women who may consider BCS without radiation therapy are those whose age $\geq 70$ years, tumor $\leq 2 \mathrm{~cm}$, tumor was completely removed, tumor contained hormone receptors, had no lymph node involvement, and received hormone therapy. Chemotherapy should be given on a case-by-case basis for women age $>70$ years, considering patient health. Lymph node removal may be optional for small tumors and for whom lymph node 
spread is unlikely, elderly women, and those with poor health. Pregnant women should not undergo radiation therapy or chemotherapy. Women who are elderly or in poor health may also be recommended to not receive chemotherapy. Choice of adjuvant hormone therapy, if needed, should consider the woman's menopausal status and future pregnancy plans. ${ }^{4,14}$

\section{$\underline{\text { Patterns of Care }}$}

In 2008, among women with early stage breast cancer (Stages I - II), 10\% had BCS only, $30 \%$ had BCS and radiation therapy, $17 \%$ had BCS, radiation therapy, and chemotherapy, $17 \%$ had mastectomy alone, $14 \%$ had mastectomy and chemotherapy, $1 \%$ had mastectomy and radiation therapy, $4 \%$ had mastectomy, radiation therapy, and chemotherapy, $6 \%$ had nonsurgical treatment, and $1 \%$ received no treatment at all. Overall, 57\% had BCS either alone or in combination with some other form of treatment, and $36 \%$ had mastectomy alone or in combination with some other treatment. Among women with late stage breast cancer (Stages III - IV), 2\% had BCS only, 1\% had BCS and radiation therapy, 10\% had BCS, radiation therapy, and chemotherapy, $7 \%$ had mastectomy only, $20 \%$ had mastectomy and chemotherapy, 2\% had mastectomy and radiation therapy, 31\% had mastectomy, radiation therapy, and chemotherapy, $18 \%$ had nonsurgical treatment, and $7 \%$ received no treatment at all. ${ }^{3}$

Variations in treatment patterns among women with breast cancer occur for a variety of reasons. It may seem intuitive that women with early stage breast cancer who have the choice of BCS vs. mastectomy would choose BCS in order to preserve their physical appearance and upper body functioning. However, over $1 / 3$ of women with early stage breast cancer receive a mastectomy. In fact, several recent studies have shown that the rates of elective mastectomy have been on the rise since about the mid 2000's among women who are candidates for BCS. ${ }^{16-18}$ Many women may choose mastectomy over BCS due to fear of side effects from radiation therapy, 
inconvenience and time to comply with the radiation therapy schedule, advances in surgical techniques for reconstructive surgery, and fear of recurrence. ${ }^{19,20}$ Similarly, higher rates of mastectomy among early stage breast cancer patients have been observed among women residing in rural locations $(60 \%$ vs. $45 \%)$, as compared to metro or urban areas. ${ }^{21,22}$ Women residing in rural areas may elect to have mastectomy over BCS because of the time and distance to travel for radiation therapy after BCS, out-of-pocket expenditures for travel for radiation therapy, opportunity cost of time off work for radiation therapy, the increased demand for coordination of care with BCS and radiation therapy, and lack of radiation oncologist in or near rural areas. ${ }^{22-24}$ Additional reasons for receipt of mastectomy vs. BCS among women with early stage breast cancer include reasons commonly associated with health disparities in general. Higher rates of mastectomy vs. BCS among early stage breast cancer patients have been observed in women who are white, or a race other than white or black, unmarried women, lower levels of education, women insured by Medicaid or uninsured, had a male surgeon, are younger than 40 or older than 70 years of age, since breast cancer is usually more aggressive among younger women, and the risks and side effects of additional and extensive treatments may outweigh the benefits for women of older age ${ }^{17,18,21,24,25}$ Women who are of low income and/or socio-demographically disadvantaged with early stage breast cancer may have higher rates of mastectomy than higher income women due to the additional costs of treatment associated with BCS. ${ }^{23,26-29}$ Similar factors have been associated with lack of lymph node biopsy among early stage breast cancer patients, lack of radiation therapy following BCS, lack of radiation therapy and chemotherapy among patients with local-regionally advanced, or later staged breast cancers, and non-initiation or early discontinuation of adjuvant hormone therapy. ${ }^{30-46}$ 


\section{$\underline{\text { Survival \& Recurrence }}$}

Five-year survival rates have improved substantially since 1975. In 1975 - 1977, the average 5-year survival rate for women of all ages and race/ethnicities in the US was $75.1 \%$ and has increased to $90.0 \%$ for $2001-2007 .{ }^{3}$ Increased survival rates can be attributed to treatment advancements and more cases diagnosed at an earlier stage due to widespread adoption of mammography screening. Additionally, in 2008, $82 \%$ of breast cancer survivors were alive after 10 years, and $77 \%$ after $15 \%$ years. ${ }^{4}$ However, survival rates vary greatly according to stage at diagnosis, age, and race/ethnicity. Five-year survival rates decrease with advancing stage at diagnosis. ${ }^{2}$ See Table 3. Similarly, breast cancer mortality tends to increase with age. ${ }^{47}$ See Table 4. Although 5-year survival rates have improved for all race/ethnicity groups, disparities persist, especially between whites and blacks. See Table 5. These differences are largely attributable to later stage at diagnosis and poorer stage-specific survival, and aggressive tumor characteristics among black women. ${ }^{4}$ Other factors associated with poor breast cancer survival are low levels of education and income, being uninsured or insured through Medicaid, receipt of guideline appropriate care, and number of comorbidities. ${ }^{48-53}$

Among women diagnosed with Stage I, II, and III breast cancer, about $11 \%$ will experience recurrence within 5 years and $20 \%$ within 10 years. Recurrence rates are higher among survivors diagnosed at advanced stages with recurrence rates of 7\% among Stage I, 11\% among Stage II, and 13\% among those diagnosed at Stage III. ${ }^{54}$ The likelihood of recurrence has been found to increase among survivors that had large tumors, poorly differentiated tumors, greater nodal involvement, estrogen receptor positive tumors, did not receive radiation therapy

after BCS, and did not receive adjuvant hormone therapy. ${ }^{54-57}$ Additionally, unhealthy lifestyle factors such as obesity, lack of physical activity, poor diet, heavy alcohol consumption, and 
smoking have been associated with poorer breast cancer survival and greater risk of recurrence. Although, it should be noted that studies of the association between diet, physical activity, and breast cancer survival and recurrence have not yielded consistent findings. ${ }^{58-66}$

\section{Statement of the Problem}

Despite advances in breast cancer treatment and survival, disparities in treatment patterns and survival outcomes persist. The most common disparities in treatment are the omission of recommended treatments and services or receiving non-preferential treatments. The most commonly omitted treatments are radiation therapy (RT) following breast-conserving surgery (BCS) or mastectomy when indicated, chemotherapy, adjuvant hormone therapy, and lymph node sampling. The appropriate course of treatment for each women is determined by tumor size, lymph node invasion, and hormone receptor status. However, when diagnosed at an early-stage, women often have an option for the type of local treatment received. Women with tumors $\leq 5 \mathrm{~cm}$ have the option of mastectomy or BCS followed by RT. Multiple randomized clinical studies have shown BCS followed by RT to be as equally effective as mastectomy for long-term survival of invasive early-stage breast cancer. ${ }^{67-70}$ Since the National Institutes of Health's (NIH) 1991 endorsement of BCS plus RT as an appropriate alternative to mastectomy for treatment of invasive early-stage breast cancer, mastectomy rates have decreased while rates of BCS plus RT have steadily increased. ${ }^{71}$ In fact, BCS, with or without RT, has become the preferred choice of primary surgical treatment among women with early-stage breast cancer, with $\sim 60 \%$ or more receiving BCS. ${ }^{18,72,73}$ Yet, in recent years, rates of mastectomy have started to slowly

increase. ${ }^{18,73,74}$ Associations with receipt of mastectomy when BCS is an option, include a desire to avoid radiation therapy due to side effects, fear of recurrence, larger tumor size, positive lymph nodes, tumors with negative hormone receptors, moderately or poorly differentiated 
tumors, lower income, being insured by Medicaid or uninsured, residing in a rural location, increased travel distance for RT, the increased cost of BCS plus RT vs. mastectomy, and surgeon characteristics that include being male, not trained in the U.S., and less recent training. ${ }^{18,73,75-79}$

In fact, many of the same factors are associated with treatment disparities at all stages of diagnosis and include age, health, socio-demographic characteristics, and access to specialty care. Age and comorbidity are two inter-related factors that are strongly associated with treatment. While increasing age has its own associated frailty, it is also associated with increasing comorbidity, that together, may make some treatments with adverse and toxic effects intolerable for some women, to the point that the harms of such treatments outweigh the benefits. ${ }^{80-85}$ In fact, clinical evidence-based treatment guidelines put forth by the National Comprehensive Cancer Network (NCCN) do not make recommendations for or against chemotherapy in women age $>70$ years, but suggest chemotherapy be given on a case-by-case basis, depending on the patient's health ${ }^{86}$ Additionally, guidelines specify that RT following BCS may be omitted among women meeting the following requirements: age $\geq 70$ years, tumors $\leq 2 \mathrm{~cm}$, node negative, estrogen-receptor positive, and receiving adjuvant hormone therapy. ${ }^{87}$ This change to recommended treatment guidelines that had previously recommended RT following BCS for all age groups, was made after the 2004 publication of the Cancer and Leukemia Group B (CALGB) C9343 trial study results were demonstrating similar 5-year survival between women with these clinical characteristics who received BCS plus RT or BCS alone. However, since this change in treatment recommendations, evidence suggests that a greater number of elderly women do not receive RT or chemotherapy, than those that meet exemption requirements from RT or who are in too poor of health to undergo chemotherapy. ${ }^{88-90}$ The course of breast cancer care is often affected by the woman's access to oncology services 
due to their location of residence and socio-demographic characteristics. Women residing in rural vs. urban areas, in areas with a lower density of oncology service providers, and that have greater distances to travel for care are more likely to not receive adjuvant treatments such as RT and chemotherapy, or delay the initiation of treatment. ${ }^{80,90-92}$ Lastly, as frequently observed with many health disparities, women who are of black or non-white Hispanic race, lower income, lower education, publically insured or uninsured are more likely to experience breast cancer treatment disparities. ${ }^{81,93,94}$

In similar fashion, these factors, as well as clinical prognostic characteristics are also associated with overall breast cancer survival. Clinical characteristics associated with greater survival are earlier stage at diagnosis, smaller tumor size, less or no lymph node invasion, well differentiated tumor grade, and estrogen and progesterone positive tumors. ${ }^{1,96-98}$ Receiving guideline-concordant care (GCC) is associated with greater breast cancer survival, while treatment disparities, i.e. guideline-discordant care (GDC), are associated with poorer survival. 99-102 While age and comorbidity are inherently associated with poorer overall survival, survival differences have also been observed between different races. Black women have markedly worse survival outcomes, as compared to white women, such that 5-year survival for white and black women is $90 \%$ and $79 \%$, respectively. ${ }^{103-105}$ Aside from being less likely to receive GCC due to various socio-demographic factors, black women diagnosed with breast cancer are more likely to have tumors with less favorable characteristics and aggressive cancers. ${ }^{106,107}$

In order to address gaps in the literature and build upon existing knowledge this series of studies will focus upon the treatment patterns and survival of elderly women with invasive breast cancer, a population with the highest incidence of the disease, but for whom many are undertreated due to age. These studies will also determine how age, health, clinical prognostic 
factors, oncology care resources, and socio-demographic characteristics are associated with these outcomes. The first study will investigate the initial type of loco-regional treatment received for early-stage breast cancer. Most studies reporting on treatment patterns for early-stage breast cancer present rates that reflects the end result of multiple surgeries, such that a woman who first had BCS with or without RT, but eventually had a mastectomy, would be classified as having a mastectomy and not BCS. This study will capture, the initial treatment, may reflect treatment preferences of elderly women with early-stage breast cancer, as many women with this diagnosis have a choice of treatment. The second study will determine to what degree women with stage I, II, and III breast cancer receive GCC according to NCCN and ASCO treatment guidelines, by comprehensively comparing clinical characteristics and the care received by each women, including individual diagnostic tests and treatment. This will address gaps in previous studies that have only studied early stages or loco-regional treatment. The third study will examine overall 5-year survival among the two previously described study populations and how each of those study outcomes (initial loco-regional treatment and receipt of GCC) and independent variables are associated with survival. Additionally, overall 5-year survival will be examined among a third, more broadly inclusive sample of women with stage I, II, III breast cancer, to gain a baseline understanding of overall survival of elderly women with invasive non-metastatic breast cancer.

Using samples of female fee-for-service Medicare beneficiaries diagnosed with breast cancer in the SEER-Medicare dataset, the goals of this study are to determine 1) rates and factors associated with receipt of BCS plus RT, mastectomy, and BCS only among elderly women with stage I and II breast cancer; 2) rates and factors associated with receipt of guideline-concordant 
care among elderly women with stage I, II, and III breast cancer; and 3) 5-year survival rates and

associated factors among elderly women with stage I, II, and III breast cancer.

\section{Specific Aims \& Objectives}

Specific Aim 1: To determine patterns of initial loco-regional treatment following an incident diagnosis of stage I and II breast cancer among female fee-for-service Medicare beneficiaries identified in the SEER-Medicare linked dataset.

Objective 1.1: To determine the proportion of women who received breast-conserving surgery plus radiation therapy, mastectomy, or breast-conserving surgery only following an incident diagnosis of stage I and II breast cancer among fee-for-service Medicare beneficiaries in the SEER-Medicare linked datasets.

Objective 1.2: To determine group differences between women who received breast-conserving surgery plus radiation therapy, mastectomy, or breast-conserving surgery only following an incident diagnosis of stage I and II breast cancer among fee-for-service Medicare beneficiaries in the SEER-Medicare linked datasets.

Objective 1.3: To determine factors associated with type of initial loco-regional treatment received following an incident diagnosis of stage I and II breast cancer among fee-for-service Medicare beneficiaries in the SEER-Medicare linked datasets.

Specific Aim 2: To determine receipt of guideline-concordant care following an incident diagnosis of pathologically staged I, II, and III breast cancer among female fee-for-service Medicare beneficiaries identified in the SEER-Medicare linked dataset.

Objective 2.1: To determine the proportion of women receiving guideline-concordant care following an incident diagnosis of pathologically staged I, II, and III breast cancer among feefor-service Medicare beneficiaries in the SEER-Medicare linked datasets.

Objective 2.2: To determine group differences between women receiving guideline-concordant vs. guideline-discordant care following an incident diagnosis of pathologically staged I, II, and III breast cancer among fee-for-service Medicare beneficiaries in the SEER-Medicare linked datasets.

Objective 2.3: To determine the factors associated with receipt of guideline-concordant care following an incident diagnosis of pathologically staged I, II, and III breast cancer among feefor-service Medicare beneficiaries in the SEER-Medicare linked datasets.

Specific Aim 3: To determine overall 5-year survival rates and associated factors following an incident diagnosis of invasive breast cancer among three different samples of female feefor-service Medicare beneficiaries identified in the SEER-Medicare linked dataset. 
Objective 3.1: To determine overall 5-year survival rates following an incident diagnosis of breast cancer and by stage among a broad sample of fee-for-service Medicare beneficiaries diagnosed at stage I, II, and III from 2003 to 2009 in the SEER-Medicare linked datasets.

Objective 3.2: To determine group differences between women who lived and died within 5 years of diagnosis of breast cancer among a broad sample of fee-for-service Medicare beneficiaries diagnosed at stage I, II, and III from 2003 to 2009 in the SEER-Medicare linked datasets.

Objective 3.3: To determine factors associated with overall 5-year survival following an incident diagnosis of breast cancer among a broad sample of fee-for-service Medicare beneficiaries diagnosed at stage I, II, and III from 2003 to 2009 in the SEER-Medicare linked datasets.

Objective 3.4: To determine overall 5-year survival rates following an incident diagnosis of breast cancer and by receipt of BCS plus RT, mastectomy, and BCS only among fee-for-service Medicare beneficiaries diagnosed at stage I and II breast cancer from 2003 to 2009 in the SEERMedicare linked datasets.

Objective 3.5: To determine group differences between women who lived and died within 5 years of diagnosis of breast cancer among fee-for-service Medicare beneficiaries diagnosed at stage I and II breast cancer from 2003 to 2009 in the SEER-Medicare linked datasets.

Objective 3.6: To determine factors associated with overall 5-year survival following an incident diagnosis of breast cancer among fee-for-service Medicare beneficiaries diagnosed at stage I and II breast cancer from 2003 to 2009 in the SEER-Medicare linked datasets.

Objective 3.7: To determine overall 5-year survival rates following an incident diagnosis of pathologically staged I, II, and III breast cancer and by receipt of GCC among fee-for-service Medicare beneficiaries diagnosed from 2004 to 2009 in the SEER-Medicare linked datasets.

Objective 3.8: To determine group differences between women who lived and died within 5 years of diagnosis of pathologically staged I, II, and III breast cancer among fee-for-service Medicare beneficiaries diagnosed from 2004 to 2009 in the SEER-Medicare linked datasets.

Objective 3.9: To determine factors associated with overall 5-year survival following an incident diagnosis of breast cancer among fee-for-service Medicare beneficiaries diagnosed from 2004 to 2009 in the SEER-Medicare linked datasets. 


\section{$\underline{\text { References }}$}

1. Howlader N, Noone A, Krapcho M, Garshell J, Miller D, Altekruse S, et al. (eds). SEER Cancer Statistics Review, 1975-2011, National Cancer Institute. Bethesda, MD, http://seer.cancer.gov/csr/1975_2011/, based on November 2013 SEER data submission, posted to the SEER web site, April 2014. Last accessed Mar 24, 2015.

2. American Cancer Society. Breast Cancer Overview. American Cancer Society, Atlanta, GA, 2012. Available at http://www.cancer.org/acs/groups/cid/documents/webcontent/003037-pdf.pdf. Last accessed Nov 28, 2012.

3. Siegel R, DeSantis C, Virgo K, et al. Cancer treatment and survivorship statistics, 2012. CA Cancer J Clin. 2012; 62: 220-241.

4. American Cancer Society. Breast Cancer Facts \& Figures 2013-2014. Atlanta: American Cancer Society, Inc. 2013. Available at: http://www.cancer.org/acs/groups/content/@ research/documents/document/acspc042725.pdf. Last accessed on Jan 19, 2015.

5. Centers for Disease Control and Prevention. Breast Cancer Basic Information: Risk Factors. Centers for Disease Control and Prevention, Atlanta, GA, 2012. Available at http://www.cdc.gov/cancer/breast/basic_info/risk_factors.htm. Last accessed Nov 28, 2012.

6. National Cancer Institute. BRCA1 and BRCA2: Cancer Risk and Genetic Testing. National Cancer Institute, Bethesda, MD, 2000. Available at http://www.cancer.gov/cancertopics/factsheet/Risk/BRCA. Last accessed Nov 28, 2012.

7. Bevers T, Anderson B, Bonaccio E, Buys S, Daly M, Dempsey P, et al. Breast Cancer Screening and Diagnosis: Clinical Practice Guidelines in Oncology. JNCCN, 2009; 7(10): 1060-1096.

8. United States Preventive Services Task Force (USPSTF). Screening for Breast Cancer: U.S. Preventive Services Task Force Recommendation Statement. Ann Intern Med, 2009; 151(10): 716-726.

9. Berg W, Gutlerrez L, NessAiver M, Carter W, Bhargavan M, Lewis R, et al. Diagnostic accuracy of mammography, clinical examination, US, and MR imaging in preoperative assessment of breast cancer. Radiology. 2004; 233(3): 830-849.

10. Bevers T, Anderson B, Bonaccio E, Buys S, Daly M, Dempsey P, et al. Breast cancer screening and diagnosis. JNCCN. 2009; 7(10): 1060-1096. 
11. National Cancer Institute. Breast Cancer Screening. National Institutes of Health, U.S. Department of Health and Human Services, Bethesda, MD, 2012. Available at http://www.cancer.gov/cancertopics/pdq/screening/breast/healthprofessional/page4. Last accessed Jan 19, 2013.

12. Breast Cancer Surveillance Consortium. Sensitivity and specificity by indication for examination for 401,572 diagnostic mammography examinations from 2002 - 2006 --based on BCSC data as of 2009. National Cancer Institute, National Institutes of Health, U.S. Department of Health and Human Services, Bethesda, MD, 2009. Available at http://breastscreening.cancer.gov/data/benchmarks/diagnostic/2009/tableSensSpec.html . Last accessed Jan 19, 2013.

13. Yankaskas B, Taplin S, Ichikawa L, Geller B, Rosenberg R, Carney P, et al. Association between mammography timing and measures of screening performances in the United States. Radiology. 2005; 234(2): 363-373.

14. National Comprehensive Cancer Network. NCCN Guidelines for Patients: Breast Cancer. Version 2.2011. National Comprehensive Cancer Network, Fort Washington PA, 2011. Available at http://www.nccn.org/patients/default.asp. Last accessed Nov 28, 2012.

15. American Cancer Society. Breast Cancer: How is Breast Cancer Staged? American Cancer Society, Atlanta, GA, 2012. Available at http://www.cancer.org/cancer/breastcancer/detailedguide/breast-cancer-staging. Last accessed Nov 28, 2012.

16. McGuire K, Santillan A, Kaur, et al. Are mastectomies on the rise? A 13-year trend analysis of the selection of mastectomy versus breast conservation therapy in 5865 patients. Ann Surg Oncol. 2009; 16(10): 2682-2690.

17. Dragun A, Pan J, Riley E, et al. Increasing use of elective mastectomy and contralateral prophylactic surgery among breast conservation candidates. Am J Clin Oncol. 2013; 36(4): 375-80.

18. Dragun A, Huang B, Tucker T, Spanos W. Increasing mastectomy rates among all age groups for early-stage breast cancer: a 10-year study of surgical choice. Breast J. 2012; 18(4): $318-325$.

19. Tate P, McGee E, Hopkins S, Rogers E, Page G. Breast conservation versus mastectomy: patient preferences in a community practice in Kentucky. J Surg Oncol. 1993; 52(4): $213-6$.

20. Balch C, Jacobs L. Mastectomies on the rise for breast cancer: "The tide is changing". Ann Surg Oncol. 2009; 16(10): 2669-2272. 
21. Markossian T, Hines R. Disparities in late stage diagnosis, treatment, and breast cancerrelated death by race, age, and rural residence among women in Georgia. Women Health. 2012; 52(4): 317-335.

22. Jacobs L, Kelley K, Rosson G, Detrani M, Chang D. Disparities in urban and rural mastectomy populations. Ann Surg Oncol. 2008; 15(10): 2644-2652.

23. Campbell J, Ramsey S. The costs of treating breast cancer in the US: A synthesis of published evidence. Pharmacoeconomics. 2009; 27(3): 199-209.

24. Schroen A, Brenin D, Kelly M, Knaus W, Slingluff C. Impact of patient distance to radiation therapy on mastectomy use in early-stage breast cancer patients. J Clin Oncol. 2005; 23(28): 7074-7080.

25. Hershman D, Buono D, McBride R, et al. Surgeon characteristics and adjuvant radiotherapy receipt after lumpectomy and after mastectomy for breast cancer. J Natl Cancer Inst. 2008; 100(3): 199-206.

26. Barlow W, Taplin S, Yoshida C, Buist D, Seger D, Brown M. Cost comparison of mastectomy versus breast-conserving therapy for early-stage breast cancer. J Natl Cancer Inst. 2001; 93(6): 447-455.

27. Warren J, Brown M, Fay M, Schussler N, Potosky AL, Riley GF. Costs of treatment for elderly women with early-stage breast cancer in fee-for-service settings. J Clin Oncol. 2002; 20(1): 307-316.

28. Palit T, Miltenburg D, Brunicardi F. Cost analysis of breast conservation surgery compared with modified radical mastectomy with and without reconstruction. Am J Surg. 2000; 179(6): 441-445.

29. Bradley C, Given C, Baser O, Gardiner J. Influence of surgical and treatment choices on the cost of breast cancer care. Eur J Health Econom. 2003; 4(2): 96-101.

30. Martinez S, Shah D, Tseng W, Canter R, Bold R. Rural-urban disparities in use of postlumpectomy radiation. Med Oncol. 2012; 29(5): 3250-3257.

31. Jagsi R, Abrahamse P, Morrow M, et al. Patterns and correlates of adjuvant radiotherapy receipt after lumpectomy and after mastectomy for breast cancer. J Clin Oncol. 2010; 28(14): 2396-2403.

32. Foley K, Kimmick G, Camacho F, Levine E, Balkrishnan R, Anderson R. Survival disadvantage among Medicaid-insured breast cancer patients treated with breast conserving surgery without radiation therapy. Breast Cancer Res Treat. 2007; 101(2): 207-214. 
33. Ramsey S, Zeliadt S, Richardson L, et al. Discontinuation of radiation treatment among Medicaid-enrolled women with local and regional stage breast cancer. Breast J. 2009; 16(1): 20-27.

34. Martinez S, Tseng W, Shah D, Canter R, Bold R. Urban and non-urban disparities in the use of post-mastectomy radiation for breast cancer. Med Oncol. 2012; 29(3): 1523-1528.

35. Dragun A, Huang B, Gupta S, Crew J, Tucker T. One decade later: trends and disparities in the application of post-mastectomy radiotherapy since the release of the American Society of Clinical Oncology Clinical Practice Guidelines. Int J Radiation Oncol Biol Phys. 2012; 83(5): e591-e596.

36. Shirvani S, Pan I, Buchholz T, et al. Impact of evidence-based clinical guidelines on the adoption of postmastectomy radiation in older women. Cancer. 2011; 117(20): 45954605.

37. Bhargava A, Du X. Racial and socioeconomic disparities in adjuvant chemotherapy for older women with lymph node-positive, operable breast cancer. Cancer. 2009; 115(13): 29993008 .

38. Freedman R, Virgo K, He Y, et al. The association of race/ethnicity, insurance status, and socioeconomic factors with breast cancer care. Cancer. 2011; 117(1): 180-189.

39. Griggs J, Hawley S, Graff J, et al. Factors associated with receipt of breast cancer adjuvant chemotherapy in a diverse population-based sample. J Clin Oncol. 2012; 30(25): 30583064 .

40. Aiello Bowles E, Boudreau D, Chubak J, et al. Patient-reported discontinuation of endocrine therapy and related adverse effects among women with early-stage breast cancer. JOP. 2012; 8(6): e149-e157.

41. Murphy C, Bartholomew L, Carpentier M, Bluethmann S, Vernon S. Adherence to adjuvant hormonal therapy among breast cancer survivors in clinical practice: a systematic review. Breast Cancer Res Treat. 2012; 134(2): 459-478.

42. Neugut A, Hillyer G, Kushi L, et al. Non-initiation of adjuvant hormonal therapy in women with hormone receptor-positive breast cancer: the breast cancer quality of care study (BQUAL). Breast Cancer Res Treat. 2012; 134(1): 419-428.

43. Friese C, Pini T, Li Y, et al. Adjuvant endocrine therapy initiation and persistence in a diverse sample of patients with breast cancer. Breast Cancer Res Treat. 2013; 138(3): 931-939.

44. Kimmick G, Anderson R, Camacho F, Bhosle M, Hwang W, Balkrishnan R. Adjuvant hormonal therapy use among insured, low-income women with breast cancer. J Clin Oncol. 2009; 27(21): 3445-3451. 
45. Halpern M, Chen A, Marlow N, Ward E. Disparities in receipt of lymph node biopsy among early-stage female breast cancer patients. Ann Surg Oncol. 2009; 16(3): 562-570.

46. Reeder-Hayes K, Baingridge J, Meyer A, et al. Race and age disparities in receipt of sentinel lymph node biopsy for early-stage breast cancer. Breast Cancer Res Treat. 2011; 128(3): 863-871.

47. Howlader N, Noone A, Krapcho M, et al. SEER Cancer Statistics Review, 1975 - 2009 (Vintage Populations). National Cancer Institute, Bethesda, MD, 2011. Available at http://seer.cancer.gov/csr/1975_2009/. Last accessed May 08, 2013.

48. Sprague B, Trentham-Dietz A, Gangnon R, et al. Socioeconomic status and survival after an invasive breast cancer diagnosis. Cancer. 2011; 117(7): 1542-1552.

49. Yu X. Socioeconomic disparities in breast cancer survival: relation to stage at diagnosis, treatment and race. BMC Cancer. 2009; 9(364).

50. McDavid K, Tucker T, Sloggett A, Coleman M. Cancer survival in Kentucky and health insurance coverage. Arch Intern Med. 2003; 163(18): 2135-2144.

51. Foley K, Kimmerick G, Camacho F, Levine E, Balkrishnan R, Anderson R. Survival disadvantage among Mediciaid-insured breast cancer patients treated with breast conserving surgery without radiation therapy. Breast Cancer Res Treat. 2007; 101(2): 207 $-214$.

52. Patnaik J, Byers T, DiGuiseppi C, Denberg T, Dabelea D. The influence of comorbidities on overall survival among older women diagnosed with breast cancer. J Natl Cancer Inst. 2011; 103(14): 1101-1111.

53. Du X, Freeman J, Nattinger A, Goodwin J. Survival of women after breast conserving surgery for early stage breast cancer. Breast Cancer Res Treat. 2002; 72(1): 23-31.

54. Mansell J, Monypenny IJ, Skene AI, et al. Patterns and predictors of early recurrence in postmenopausal women with estrogen receptor-positive early breast cancer. Breast Cancer Res Treat. 2009; 117(1): 91-98.

55. Brewster A, Hortobagyi G, Broglio K, et al. Residual risk of breast cancer recurrence 5 years after adjuvant therapy. J Natl Cancer Inst. 2008; 100(16): 1179-1183.

56. Early Breast Cancer Trialists' Collaborative Group (EBCTCG). Effect of radiotherapy after breast- conserving surgery on 10-year recurrence and 15-year breast cancer death: metaanalysis of individual patient data for 10801 women in 17 randomised trials. Lancet. 2011; 378(9804): 1707-1716.

57. Bosco J, Last T, Prout M, et al. Breast cacner recurrence in older women five to ten years after diagnosis. Cancer Epidemiol Biomarkers Prev. 2009; 18(11): 2979-2983. 
58. Braithwaite D, Izano M, Moore D, et al. Smoking and survival after breast cancer diagnosis: a prospective observational study and systematic review. Breast Cancer Res Treat. 2012; 136(2): 521-533.

59. Peirce J. Diet and breast cancer prognosis: making sense of the WHEL and WINS trials. Curr Opin Obstet Gynecol. 2009; 21(1): 86-91.

60. Irwin M, McTiernan A, Manson J, et al. Physical activity and survival in postmenopausal women with breast cancer: results from the Women's Health Initiative. Cancer Prev Res. 2011; 4(4): 522-529.

61. Ibrahim E, Al-Homaidh A. Physical activity and survival after breast cancer diagnosis: meta-analysis of published studies. Med Oncol. 2011; 28(3): 753-765.

62. Kwan M, Kushi L, Weltzien E, et al. Alcohol consumption and breast cancer recurrence and survival among women with early-stage breast cancer: the Life After Cancer Epidemiology Study. J Clin Oncol. 2010; 28(29): 4410-4416.

63. Protani M, Coory M, Martin J. Effect of obesity on survival of women with breast cancer: systematic review and meta-analysis. Breast Cancer Res Treat. 2010(3); 123: 627-635.

64. Thomson C, Rock C, Thompson $\mathrm{P}$, et al. Vegetable intake is associated with reduced breast cancer recurrence in tamoxifen users: a secondary analysis from the Women's Healthy Eating and Living Study. Breast Cancer Res Treat. 2011; 125(2): 519-527.

65. Kroenke C, Chen W, Rosner B, Holmes M. Weight, weight gain, and survival after breast cancer diagnosis. J Clin Oncol. 2005; 23(7): 1370-1378.

66. Patterson R, Cadmus L, Emond J, Pierce J. Physical activity, diet, adiposity and female breast cancer prognosis: a review of the epidemiologic literature. Maturitas. 2010; 66(1): $5-15$.

67. Fisher B, Anderson S, Bryant J, Margolese R, Deutsch M, Fisher E, et al. Twenty-year follow-up trial comparing total mastectomy, lumpectomy, and lumpectomy plus irradiation for the treatment of invasive breast cancer. N Engl J Med. 2002; 347(16): $1233-241$.

68. Veronesi U, Cascinelli N, Mariani L, Greco M, Saccozzi R, Luini A, et al. Twenty-year follow-up of a randomize study comparing breast-conserving surgery with radical mastectomy for early breast cancer. N Engl J Med. 2002; 347(16): 1227-1232.

69. Litière S, Werutsky G, Fentiman I, Rutgers E, Christiaens M, Limbergen E, et al. Breast conserving therapy versus mastectomy for stage I-II breast cancer: 20 year follow-up of the EORTC 10801 phase 3 randomised trial. Lancet Oncol. 2012; 13(4): 412-19. 
70. Simone N, Dan T, Shih J, Smith S, Sciuto L, Lita E, et al. Twenty-five year results of the national cancer institute randomized breast conservation trial. Breast Cancer Res Treat. 2012; 132(1): 197-203.

71. NIH Consensus Conference. Treatment of early-stage breast cancer. JAMA. 1991; 265(3): 391-395.

72. American Cancer Society. Cancer Treatment and Survivorship Facts \& Figures 2014-2015. Atlanta: American Cancer Society; 2014.

73. Mahmood U, Hanlon A, Koshy M, Buras R, Chumsri S, Tkaczuk K, et al. Increasing national mastectomy rates for the treatment of early-stage breast cancer. Ann Surg Oncol. 2013; 20(5): 1436-1443.

74. McGuire K, Santillan A, Kaur P, Meade T, Parbhoo J, Mathias M, et al. Are mastectomies on the rise? A 13-year trend analysis of the selection of mastectomy versus breast conservation therapy in 5865 patients. Ann Surg Oncol. 2009; 16(10): 2682-2690.

75. Boscoe F, Johnson C, Henry K, Goldberg D, Shahabi K, Elkin E, et al. Geographic proximity to treatment for early-stage breast cancer and likelihood of mastectomy. Breast. 2011; 20(4): 324-328.

76. Goyal S, Chandwani S, Haffty B, Demissie K. Effect of travel distance and time to radiotherapy on likelihood of receiving mastectomy. Ann Surg Oncol. 2014; Sept 23: Epub ahead of print.

77. Bradley C, Given C, Baser O, Gardiner J. Influence of surgical and treatment choices on the cost of breast cancer care. Eur J Health Econ. 2003; 4(2): 96-101.

78. Jacobs L, Kelley K, Rosson G, Detrani M, Chang D. Disparities in urban and rural mastectomy populations: the effects of patient- and county-level factors on likelihood of receipt of mastectomy. Ann Surg Oncol. 2008; 15(10): 2644-2652.

79. Schroen A, Brenin D, Kelly M, Knaus W, Slingluff C. Impact of patient distance to radiation therapy on mastectomy use in early-stage breast cancer patients. J Clin Oncol. 2005; 23(28): 7074-7080.

80. Dragun A, Huang B, Tucker T, Spanos W. Disparities in the application of adjuvant radiotherapy after breast-conserving surgery for early-stage breast cancer: impact on overall survival. Cancer. 2011; 117(12): 2590-2598.

81. Bhargava A \& Du X. Racial and socioeconomic disparities in adjuvant chemotherapy for older women with lymph node-positive, operable breast cancer. Cancer. 2009; 115(13): 2999-3008. 
82. Nuegut A, Hillyer G, Kushi L, Lamerato L, Nathanson S, Ambrosone C, et al. The Breast Cancer Quality of Care Study (BQUAL): A Multi-Center Study to Determine Causes for noncompliance with Breast Cancer Adjuvant Therapy. Breast J. 2012; 18(3): 203-213.

83. Yarnold J, Ashton A, Bliss J, Homewood J, Harper C, Hanson J, et al. Fractionation sensitivity and dose response of late adverse effects in the breast after radiotherapy for early breast cancer: long-term results of a randomised trial. Radiother Oncol. 2005; 75(1):9-17.

84. Landoni V, Giordano C, Marsella A, Saracino B, Petrongari M, Ferraro A, et al. Evidence from a breast cancer hypofractionated schedule: late skin toxicity assessed by ultrasound. J Exp Clin Cancer Res. 2013; 32(80).

85. Reinisch M, Minckwitz G, Harbeck N, Janni W, Kümmel S, Kaufmann M, et al. Side Effects of Standard Adjuvant and Neoadjuvant Chemotherapy Regimens According to Age Groups in Primary Breast Cancer. Breast Care. 2013; 8(1): 60-66.

86. Carlson R, Allred D, Anderson B, Burstein H, Carter W, Edge S, et al. Breast cancer. Clinical practice guidelines in oncology. J Natl Compr Canc Netw. 2009; 7(2):122-192.

87. Hughes K, Schnaper L, Berry D, Cirrincione C, McCormick B, Shank B, et al. Lumpectomy plus tamoxifen with or without irradiation in women 70 years of age or older with early breast cancer. N Engl J Med. 2004; 351(10): 971-977.

88. McCormick B, Ottesen R, Hughes M, Javid S, Khan S, Mortimer J, et al. Impact of Guideline Changes on Use or Omission of Radiation in the Elderly with Early Breast Cancer: Practice Patterns at National Comprehensive Cancer Network Institutions. J Am Coll Surg. 2014; 219(4):796-802.

89. Mackey R, Chandru Kowdley G. Treatment practices and outcomes of elderly women with breast cancer in a community hospital. Am Surg. 2014; 80(7): 714-719.

90. Wheeler S, Carpenter W, Peppercorn J, Schenck A, Weinberger M, Biddle A. Predictors of timing of adjuvant chemotherapy in older women with hormone receptor-negative, stages II-III breast cancer. Breast Cancer Res Treat. 2012; 131(1): 207-216.

91. Martinez S, Shah D, Tseng W, Canter R, Bold R. Rural-urban disparities in use of postlumpectomy radiation. Med Oncol. 2012; 29(5): 3250-3257.

92. Hershman D, Wang X, McBride R, Jacobson J, Grann V, Neugut A. Delay of adjuvant chemotherapy initiation following breast cancer surgery among elderly women. Breast Cancer Res Treat. 2006; 99(3): 313-321.

93. Freedman R, Virgo K, He Y, Pavluck A, Winer E, Ward E, et al. The Association of Race/Ethnicity, Insurance Status, and Socioeconomic Factors With Breast Cancer Care. 2010; 117(1): 180-189. 
94. Wu X, Lund M, Kimmick G, Richardson L, Sabatino S, Chen V, et al. Influence of race, insurance, socioeconomic status, and hospital type on receipt of guideline-concordant adjuvant systemic therapy for locoregional breast cancers. J Clin Oncol. 2012; 30(2): $142-150$.

95. Berry D, Cronin K, Plevritis S, Fryback D, Clarke L, Zelen M, et al. Effect of screening and adjuvant therapy on mortality from breast cancer. N Engl J Med. 2005; 353(17): 17841792.

96. Rosenberg J, Chia Y, Plevritis S. The effect of age, race, tumor size, tumor grade, and disease stage on invasive ductal breast cancer survival in the U.S. SEER database. Breast Cancer Res Treat. 2005; 89(1): 47-54.

97. Fisher B, Redmond C, Fisher E, Caplan R. Relative worth of estrogen or progesterone receptor and pathologic characteristics of differentiation as indicators of prognosis in node negative breast cancer patients: findings from National Surgical Adjuvant Breast and Bowel Project Protocol B-06. J Clin Oncol. 1988; 6(7):1076-1087.

98. Hwang E, Lichtensztajn D, Gomez S, Fowble B, Clarke C. Survival after lumpectomy and mastectomy for early-stage invasive breast cancer: the effect of age and hormone receptor status. Cancer. 2013; 119(7): 1402-1411.

99. Tuttle T, Jarosek S, Habermann E, Yee D, Yuan J, Virnig B. Omission of radiation therapy after breast-conserving surgery in the United States. A Population-Based Analysis of Clinicopathologic Factors. Cancer. 2012; 118(8): 2004-2013.

100. Sun S, Hollenbeak C, Leung A. Deviation from the Standard of Care for Early Breast Cancer in the Elderly: What are the Consequences? Ann Surg Oncol. 2014; Dec 17: DOI 10.1245/s10434-014-4290-5 [Epub ahead of print].

101. Giordano S, Duan Z, Kuo Y, Hortobagyi G, Goodwin J. Use and Outcomes of Adjuvant Chemotherapy in Older Women With Breast Cancer. J Clin Oncol. 2006; 24(18): 2750 2756.

102. Javid S, Varghese T, Morris A, Porter M, He H, Buchwald D, et al. Guideline-concordant cancer care and survival among American Indian/Alaskan Native patients. Cancer. 2014; 120(14): 2183-2190.

103. American Cancer Society. Cancer Facts \& Figures 2015. Atlanta: American Cancer Society; 2015. Available at: http://www.cancer.org/acs/groups/content/@editorial/documents/document/acspc044552.pdf. Last accessed on Jan 19, 2015.

104. Iqbal J, Ginsburg O, Rochon P, Sun $\mathrm{P}^{1}$, Narod S. Differences in breast cancer stage at diagnosis and cancer-specific survival by race and ethnicity in the United States. JAMA. 2015; 313(2): 165-173. 
105. Harper S, Lynch J, Meersman S, Breen N, Davis W, Reichman M. Trends in areasocioeconomic and race-ethnic disparities in breast cancer incidence, stage at diagnosis, screening, mortality, and survival among women ages 50 years and over (1987-2005). Cancer Epidemiol Biomarkers Prev. 2009; 18(1): 121-131.

106. Maskarinec G, Sen C, Koga K, Conroy S. Ethnic differences in breast cancer survival: status and determinants. Womens Health (Lond Engl). 2011; 7(6): 677-687.

107. Cunningham J, Butler W. Racial disparities in female breast cancer in South Carolina: clinical evidence for a biological basis. Breast Cancer Res Treat. 2004; 88(2): 161-176. 
Table 1. Definition of "T", "N", and "M" Categories of the TNM Staging System

\begin{tabular}{|l|l|l|}
\hline \multicolumn{3}{|c|}{ Breast Cancer Staging Categories } \\
\hline $\begin{array}{l}\text { Tis: non-invasive breast } \\
\text { cancer (DCIS of LCIS) }\end{array}$ & $\begin{array}{l}\text { N0: cancer has not spread to } \\
\text { lymph nodes }\end{array}$ & M0: no distant cancer spread \\
\hline $\begin{array}{l}\text { T0: no evidence of primary } \\
\text { tumor }\end{array}$ & $\begin{array}{l}\text { N1: cancer has spread to 1-3 } \\
\text { lymph nodes under the arm on } \\
\text { the same side but are not } \\
\text { attached }\end{array}$ & $\begin{array}{l}\text { M1: cancer has spread to } \\
\text { distant organs }\end{array}$ \\
\hline $\begin{array}{l}\text { T1: tumor } \leq \mathbf{2} \mathbf{~ c m} \text { in } \\
\text { diameter }\end{array}$ & $\begin{array}{l}\text { N2: cancer has spread to 4-9 } \\
\text { lymph nodes under the arm on } \\
\text { the same side and are attached }\end{array}$ & \\
\hline $\begin{array}{l}\text { T2: } \mathbf{2} \text { cm }<\text { tumor } \leq \mathbf{5} \mathbf{c m} \text { in } \\
\text { diameter }\end{array}$ & $\begin{array}{l}\text { N3: cancer has spread to 10 or } \\
\text { more lymph nodes including } \\
\text { those under the arm and } \\
\text { surrounding areas on the same } \\
\text { side }\end{array}$ & \\
\hline $\begin{array}{l}\text { T3: tumor }>\mathbf{5} \text { cm in } \\
\text { diameter }\end{array}$ & & \\
\hline $\begin{array}{l}\text { T4: tumor is any size, but } \\
\text { has spread to the chest wall } \\
\text { or skin }\end{array}$ & & \\
\hline
\end{tabular}

Table 2. TNM Staging System for Breast Cancer

\begin{tabular}{|c|c|c|c|}
\hline \multicolumn{5}{|c|}{ Breast Cancer Stages } \\
\hline Overall Stage & T Category & N Category & M Category \\
\hline Stage 0 & Tis & N0 & M0 \\
\hline Stage I & T1 & N0 & M0 \\
\hline Stage IIA & T0 & N1 & M0 \\
& T1 & N1 & M0 \\
\hline Stage III & T2 & N0 & M0 \\
& T2 & N1 & M0 \\
\hline Stage IIIA & T3 & N0 & M0 \\
& T1 & N2 & M0 \\
& T2 & N2 & M0 \\
& T3 & N1 & M0 \\
\hline Stage IIIB & T3 & N2 & M0 \\
\hline Stage IIIC & Any T & Any N & M0 \\
\hline Stage IV & Any T & Any N & M0 \\
\hline & & & M1 \\
\hline
\end{tabular}


Table 3. Breast Cancer Survival, 2001 - 2007

\begin{tabular}{|c|c|}
\hline \multicolumn{2}{|c|}{$\begin{array}{l}\text { 5-Year Breast Cancer Survival } \\
\text { Rates by Stage at Diagnosis }\end{array}$} \\
\hline Stage & Rate \\
\hline 0 & $93 \%$ \\
\hline I & $88 \%$ \\
\hline IIA & $81 \%$ \\
\hline IIB & $74 \%$ \\
\hline IIIA & $67 \%$ \\
\hline IIIB & $41 \%$ \\
\hline IIIC & $49 \%$ \\
\hline IV & $15 \%$ \\
\hline
\end{tabular}

Table 4. Breast Cancer Mortality by Age, $2005-2009$

\begin{tabular}{|l|l|}
\hline \multicolumn{2}{|c|}{$\begin{array}{c}\text { Average Annual } \\
\text { Mortality } \\
\text { Rate by Age }\end{array}$} \\
\hline \multicolumn{1}{|c|}{ Age } & \multicolumn{2}{c|}{ Rate } \\
\hline$<20$ years & $0.0 \%$ \\
\hline $20-34$ years & $0.9 \%$ \\
\hline $35-44$ years & $5.6 \%$ \\
\hline $45-54$ years & $14.8 \%$ \\
\hline $55-64$ years & $21.4 \%$ \\
\hline $65-74$ years & $19.9 \%$ \\
\hline $75-84$ years & $22.0 \%$ \\
\hline$\geq 85$ years & $15.5 \%$ \\
\hline
\end{tabular}

Table 5. Breast Cancer Survival by Race/Ethnicity, 2001 - 2007

\begin{tabular}{|l|l|}
\hline \multicolumn{2}{|c|}{$\begin{array}{r}\text { 5-Year Breast Cancer Survival Rates } \\
\text { by Race/Ethnicity }\end{array}$} \\
\hline \multicolumn{1}{|c|}{ Race/Ethnicity } & \multicolumn{2}{c|}{ Rate } \\
\hline Non-Hispanic White & $88.8 \%$ \\
\hline Black & $77.5 \%$ \\
\hline AI/AN & $85.6 \%$ \\
\hline Asian & $90.7 \%$ \\
\hline Pacific Islander & $85.4 \%$ \\
\hline Hispanic & $83.8 \%$ \\
\hline
\end{tabular}


CHAPTER 2 


\section{CHAPTER 2}

"Variations in Initial Loco-Regional Treatment Received for Early-Stage Breast Cancer among Elderly Women in a SEER-Medicare Population: Associations with Old Age and Vulnerable Socio-Demographics"

Traci LeMasters ${ }^{1}$

S. Suresh Madhavan, M.B.A, Ph.D. Chair ${ }^{1}$

Usha Sambamoorthi Ph.D. ${ }^{1}$

Kimberly M Kelly Ph.D. ${ }^{1,2}$

Hannah Hazard M.D. ${ }^{3}$

Dustin Long Ph.D. ${ }^{4}$

1 Department of Pharmaceutical Systems and Policy, School of Pharmacy, West Virginia University

2 Mary Babb Randolph Cancer Center, West Virginia University

${ }^{3}$ Department of Medical Education, School of Medicine, West Virginia University

${ }^{4}$ Department of Biostatistics, School of Public Health, West Virginia University 


\begin{abstract}
Background: Breast-conserving surgery (BCS) plus radiation therapy $(\mathrm{RT})$ is an equally effective loco-regional treatment for long-term early-stage breast cancer survival as mastectomy, and is often the preferred choice of treatment by the majority of women. While some women may eventually require mastectomy after treatment failure with BCS plus RT, a considerable number of elderly women receive mastectomy as their initial loco-regional treatment or do not receive $\mathrm{RT}$ following BCS. Therefore, the purpose of this study was to examine patterns of initial loco-regional treatment (BCS plus RT, mastectomy, or BCS without RT) among elderly women with early-stage breast cancer and differences between women who received mastectomy or BCS without RT, compared to BCS plus RT as initial loco-regional treatment.
\end{abstract}

Methods: A sample of 45,981 women age $\geq 66$ diagnosed with stage I and II breast cancer from 2003 to 2009 were selected from the SEER-Medicare linked dataset. The chi-square statistic was used to test for group differences between type of treatment and independent variables that included year of diagnosis, age, health, clinical prognostic factors, oncology care resources, and socio-demographic characteristics. Multinomial logistic regression was used to identify predictors of mastectomy or BCS without RT, compared to BCS plus RT for initial loco-regional treatment.

Results: In all, 55\% of elderly women with early-stage breast cancer received BCS plus RT, $23 \%$ received mastectomy, and $22 \%$ received BCS without RT as their initial type of locoregional treatment. Compared to women who received BCS plus RT, women of older age, greater comorbidity, with a higher frequency of PCP visits, later stage, and non-white race were more likely to receive mastectomy or BCS without RT. Whereas women who lived in areas of higher education, higher income, metro areas, and were treatment by an oncology surgeon or 
both and oncology and general surgeon were less likely to receive mastectomy or BCS without RT, than BCS plus RT. Additionally, women who resided in areas with a high density of oncology centers were less likely to receive mastectomy, while women with negative estrogen and progesterone tumor receptors and less differentiated tumors were more likely to receive mastectomy, compared to BCS plus RT.

Conclusions: Over half of elderly women have BCS plus RT, while the other half undergo mastectomy or have BCS without RT, in nearly equal proportions. Yet, many may eventually undergo mastectomy after initial treatment with BCS. Elderly women who were more likely to have mastectomy or BCS without RT share similar associations with many of the same sociodemographic characteristics and measures of access to oncology care, indicating they face additional barriers to receiving preferential and/or optimal treatment. Moreover, positive associations with increasing age and comorbidity among women who received mastectomy or BCS without RT may reflect a notion that RT is not well tolerated or will not significantly increase life-expectancy among some elderly women. Loco-regional treatment of elderly women with early-stage breast cancer could be improved by ensuring all patients are informed of and have access to all treatment options, as well as, emphasizing receipt of all recommended treatments among patients who do not meet specific criteria for exemption. 


\section{Introduction}

Breast conserving surgery (BCS) followed by radiation therapy (RT), has been found to be an equally effective loco-regional treatment as mastectomy for long-term survival of invasive early-stage breast cancer. ${ }^{1-5}$ Since the National Institutes of Health's (NIH) 1991 endorsement of BCS plus RT as an appropriate alternative to mastectomy for treatment of invasive early-stage breast cancer, mastectomy rates have decreased while rates of BCS plus RT have steadily increased. ${ }^{6}$ In fact, BCS, with or without RT, has become the preferred choice of primary surgical treatment among women with early-stage breast cancer, with $\sim 60 \%$ or more receiving BCS. ${ }^{7-9}$ Yet, one in four women who have BCS will undergo additional surgery, and about one in ten will eventually undergo mastectomy after treatment failure with BCS. ${ }^{10,11}$ Whether it is the initial choice of surgery or not, epidemiological studies have reported an increase in the use of mastectomy in recent years. ${ }^{8,9,12}$ Women who elect for mastectomy when BCS plus RT is an option tend to be younger, have a desire to avoid RT, fear recurrence, have larger and/or more aggressive tumors, positive lymph nodes, tumors with negative hormone receptors, and moderately or poorly differentiated tumors. Additional associations with receipt of mastectomy are being of lower income, insured by Medicaid or uninsured, residing in a rural location, increased travel distance for RT, the increased cost of BCS plus RT compared to mastectomy, and surgeon characteristics that include being male, not trained in the U.S., and less recent

training. ${ }^{8,9,12-17}$ In fact, many of these same factors are similarly associated with omission of RT following BCS. Additional associations with not receiving RT following BCS include being African American, increased comorbidity, and older age. ${ }^{18-21}$

Among several issues to consider when choosing local treatment for early-stage breast cancer, is a willingness to undergo RT when opting to have BCS. Omitting RT following BCS 
may result in short-term treatment failure, and consequently, mastectomy. Moreover, women who do not received RT following BCS when recommended to do so are at increased risk for recurrence and poorer survival. ${ }^{18,22-26}$ However, guidelines allow for a select group of women to omit RT following BCS. Following the 2004 publication of the Cancer and Leukemia Group B (CALGB) C9343 trial study results demonstrating similar 5-year survival between women age $\geq$ 70 years with specific clinical characteristics who received BCS plus RT or BCS alone, the National Comprehensive Cancer Network's (NCCN) updated treatment guidelines to allow for the omission of RT following BCS among women meeting the following requirements: age $\geq 70$ years, tumors $\leq 2 \mathrm{~cm}$, node negative, estrogen-receptor positive, and receiving adjuvant hormone therapy. ${ }^{27,28}$ Following this controversial guideline change, rates RT following BCS appeared to decrease, particularly among women age $\geq 80$ years regardless of clinical factors or evidence of increased breast cancer mortality and recurrence among women receiving BCS only. ${ }^{18,22-25,29,30}$

\section{Statement of the Problem}

Until now, epidemiological studies of treatment for early-stage breast cancer have included women of all ages or have limited studies of older women with stage I breast cancer or who would already meet NCCN guidelines for RT omission. ${ }^{8,9,12,13,22-24,31,33}$ Samples have also included women with Stage 0, for which surgical guidelines are not clear, or women with Stage III, a stage where mastectomy is the predominant surgical choice. ${ }^{12,22,31,33}$ Moreover, when reporting surgical patterns, studies have included mastectomy when it may not have been the initial surgery. ${ }^{34}$ Therefore, to gain a better understanding of the intended initial choice of treatment among elderly women with invasive early-stage breast cancer, this study examine patterns of initial loco-regional treatment (BCS plus RT, mastectomy, or BCS only) among women age $\geq 66$ years, with stage I and I breast cancer using SEER using a U.S. population- 
based linked SEER-Medicare dataset. Furthermore, this study aims to determine how age, health, clinical prognostic factors, oncology care resources, and socio-demographic characteristics are associated with initial choice of loco-regional treatment.

\section{Methods}

$\underline{\text { Data }}$

The SEER-Medicare database was created in a collaborative effort by the National Cancer Institute (NCI) and Centers for Medicare and Medicaid Services (CMS) to combine clinical information gathered by the Surveillance Epidemiology and End Results cancer registry program with Medicare insurance claims. The SEER program collects basic socio-demographic, clinical, and vital status information on incident cancer cases from multiple reporting sources. This information is found the Patient Entitlement and Diagnosis Summary File (PEDSF). SEER data used for this study consisted of 17 population-based registries, representing $26 \%$ of the U.S. population. An algorithm based on name, Social Security number, sex, and date of birth successfully matched $94 \%$ SEER cases to their Medicare claims beginning from the time of eligibility till death. ${ }^{35}$ This study used Medicare claims providing information regarding beneficiary enrollment (Denominator File), hospital inpatient claims (Med-PAR file), hospital outpatient claim (Outpatient file), physician office visits (NCH file), and durable medical equipment (DME file). ${ }^{36}$ Additionally, county and state of diagnosis were used to identify the area-level density of mammography screening and oncology centers from the U.S. Department of Health and Human Resource's 2009 Area Resource File (ARF). ${ }^{37}$

\section{$\underline{\text { Study Population }}$}

This retrospective cohort study selected American Joint Commission on Cancer (AJCC) staged I and II incident cases of breast cancer recorded in SEER during years 2003 to 2009, 
among female age-eligible Medicare beneficiaries who were continuously enrolled in Medicare Part A and B fee-for-service programs 1 year before and after diagnosis, and were therefore age $\geq 66$ years at the time of diagnosis. Women were excluded if they were enrolled in a managed care program at any time during year before or the year after diagnosis, diagnosed at the time of death or upon autopsy, died within the first year of diagnosis, had end-stage renal disease, did not have primary surgical treatment, or surgeon specialty was missing. The final sample size was $\mathrm{n}=45,981$ elderly women.

\section{$\underline{\text { Measures }}$}

Dependent Variable

The dependent variable of interest was the initial loco-regional treatment received for early-stage breast cancer (BCS plus RT, mastectomy, or BCS only) identified using surgical and radiation claims where the claim date was no later than 366 days from the date of diagnosis. Surgery claims were taken from the MEDPAR, NCH, and Outpatient files using ICD-9 procedure codes 85.20 - 85.29 and CPT/HCPCS 19120, 19125, 19126, 19160, 19162, 19301, 19302 to identify BCS (i.e. lumpectomy, partial mastectomy, segmental mastectomy) and ICD-9 procedure codes 85.33 - 85.48 and CPT/HCPCS 19140, 19180, 19182, 19300, 19303, 19304, 19200, 19220, 19240, 19305, 19306, 19307, 19260, 19271, 19272 to identify mastectomy. When multiple claims for lumpectomy were present, or when a claim for lumpectomy was followed by a claim for mastectomy, only the first surgical claim was used, to identify initial choice for definitive surgical treatment. Receipt of RT was identified from MEDPAR, NCH, Outpatient, and DME files using ICD-9 diagnosis codes V580,V661,V671, ICD-9 procedure codes 9220 9239, and HCPCS codes 77261 - 77799, G0256, G0261, G0173, G0174, G0243, G0251, G0338G03340. Only the first claim was used to identify the initiation of RT. 
Independent Variables

This study examined the relationship between primary treatment and year of diagnosis, age, health, clinical prognostic factors, oncology care resources, and socio-demographic characteristics. Year of diagnosis (2003 thru 2009) was included in analytic models to assess differences in treatment over time, considering the NCCN's change in recommended treatment for early-stage breast cancer among elderly women during this time period.

Age \& Health

Age at diagnosis $(66-69,70-74,75-79, \geq 80)$ and health were considered together since declining health associated with increasing age is the reason given by NCCN guidelines for caseby-case use of chemotherapy in women older than 70 years, and are often cited as associations with undertreatment among elderly women in retrospective studies. Health was measured by calculating patient comorbidity by applying an algorithm to Medicare claims that identifies a comorbid condition as the presence of at least one in-patient or two out-patient claims using ICD-9 diagnoses codes for each co-morbid condition. Overall comorbidity was then classified

using the Klabunde adaption of the Charlson comorbidity index score $(0,1, \geq 2) \cdot{ }^{38,39}$ Frequency of primary care provider (PCP) visits was also used as a proxy for health status, with a higher number of visits considered an indication of poorer health. An index of PCP visit intensity was identified by counting of number of unique PCP claim dates the year before diagnosis in the $\mathrm{NCH}$ file. PCP visits were categorized by lower and upper 50th percent median cutoff (low, high).

\section{Clinical Prognostic Factors}

Clinical prognostic factors examined were stage at diagnosis, estrogen receptor (ER) tumor status (positive, negative, borderline/unknown), progesterone receptor (PR) tumor status 
(positive, negative, borderline/unknown), and tumor grade (well differentiated, moderately differentiated, poorly differentiated, undifferentiated/unknown). Stage diagnosis was categorized using the AJCC Stage Group 6th edition (I, II).

\section{Oncology Care Resources}

Patient's access to oncology care resources was measured as the area-level density of mammography screening and oncology treatment centers where they resided and the specialty of the surgeon that provided their surgical treatment. The density of area-level mammography screening centers and oncology treatment centers, relative to each women, was measured using data from the ARF and categorized in similar fashion (low, high). Surgeon specialty was assessed using provider specialty claims codes 02, 49 (general) and 83, 90, 91, 98 (oncology) from the NCH file to determine the type of surgeon seen (general only, oncology only, both). Socio-Demographic Characteristics

Demographic characteristics examined were race (white, other), education (measured by the 2000 Census tract survey of percent of persons age $\geq 25$ with at least 4 years college education) $(<15 \%, \geq 15 \%)$, and annual income (measured by the 2000 Census tract survey of median income by census $)(\leq \$ 35,000,>\$ 35,000)$. Location of residence was measured as metro status (non-metro, metro).

\section{$\underline{\text { Analysis }}$}

Descriptive analysis was performed for all study variables. Mantel-Haenszel chi-square tests of location shift using modified ridit scores or table scores and Mantel-Haenszel chi-square tests of general association (depending on whether the independent measure was dichotomous, nominal, or ordinal) were used to compare significant group differences between type of primary treatment and independent measures, with significance set at $P \leq .05$. The probability of 
receiving a type of primary treatment was estimated using multinomial logistic regression models for each of the independent variables described above. Parameter estimates calculated in the regression models are presented as adjusted odds ratios (AOR) with their corresponding 95\% confidence intervals (CI). All analysis were conducted using SAS version 9.4 software (SAS Institute Inc., Cary, NC).

\section{Results}

$\underline{\text { Population Characteristics }}$

In the years of 2003 thru 2009, a total of 45,981 fee-for-service female Medicare beneficiaries age $\geq 66$ years were diagnosed with Stage I (62.9\%) and Stage II (37.1\%) breast cancer. The majority were treated with BCS plus RT (54.5\%), while the rest received mastectomy (23.4\%) and BCS only (22.1\%) in similar proportions, and the majority saw both general and oncology surgeons (75.6\%). In this sample $89.4 \%$ of the women were white, $70.5 \%$

lived in an area where greater than $15 \%$ of the population was college educated, $76.4 \%$ in an area with an average annual income $>\$ 35,000$, and $83.3 \%$ lived in a metro area (Table 1 ).

Group Differences by Treatment

All group differences by treatment were significant at the $P<.001$ level (Table 2). Rates of BCS plus RT for among elderly women with invasive early-stage breast cancer increased steadily from $52.8 \%$ in 2003 to $56.4 \%$ in 2009 , while mastectomy rates decreased from $24.8 \%$ to $21.9 \%$ in 2006 and then increased to $24.3 \%$ in 2009. Similar to mastectomy, rates of BCS without RT increased from $22.3 \%$ in 2003 to $24.4 \%$ in 2005 , and then decreased to $19.3 \%$ in 2009. 
Age \& Health

Rates of BCS plus RT were the highest among women age 66 - 69 years at $65.0 \%$ and lowest for those age $\geq 80$ years $(38.3 \%)$. In contrast, rates of mastectomy and BCS without RT were the highest among those age $\geq 80$ years $(27.7 \% \& 34.0 \%)$ and lowest among women age 66 - 69 years $(20.0 \% \& 15.0 \%)$. Women with a comorbidity score $=0$ had the highest rates of BCS plus RT (57.5\%), whereas women with a comorbidity score $=2$ had the lowest rates of BCS plus RT 9 47.8\%). Conversely, mastectomy and BCS with RT rates were the highest among women with a comorbidity score $=2(25.6 \% \& 26.6 \%)$, and the lowest for women with no comorbidity (22.6\% \& 19.9\%). Possibly reflective of comorbidity, women with a low frequency of PCP visits had higher rates of BCS plus RT (57.0\%), while women with a high frequency of PCP visits had higher rates of mastectomy and BCS without RT (24.0\% \& $24.4 \%)$.

\section{Clinical Prognostic Factors}

Women with favorable prognostic clinical characteristics also had the highest rates of BCS plus RT. Women who were stage I, had ER and PR positive tumors, and well-differentiated tumors had the highest rates of BCS plus RT $(61.4 \%, 56.7 \%, 57.1 \%, 58.8 \%)$, than women who were stage II, had borderline/unknown ER and PR tumors, and undifferentiated tumors (42.9\%, $39.5 \%, 40.8 \%$, and $46.8 \%$, respectively). In comparison, women with poor prognostic factors had the highest rates of mastectomy and BCS without RT, and the lowest rates of BCS plus RT. Oncology Care Resources

Those who resided in area with a high density of mammography screening and oncology treatment centers had higher rates of BCS plus RT, while those who resided in low density areas had higher rates of mastectomy and BCS without RT. Women who were treated by a general surgeon had the lowest rates o BCS plus RT (42.9\%), while those treated by a oncology surgeon 
or both types of surgeons had higher rates of BCS plus RT (59.0\% \& 56.2\%). In contrast, women treated by a general surgeon had higher rates of BCS without RT (33.8\%), and those who saw an oncology surgeon or both had similarly lower rates $(19.0 \% \& 19.4 \%)$.

Socio-demographic Characteristics

Treatment differences by socio-demographic characteristics were in the expected direction with women who were of white race, lived in areas of greater education, higher income, and of metro status having higher rates of BCS plus RT $(55.2 \%, 57.8 \%, 57.5 \%, 56.7 \%)$, than did women of other race, lived in less educated, lower income, and non-metro areas $(48.5 \%, 46.6 \%$, $45.5 \%, 43.8 \%$, respectively). Comparatively, women of other race, lived in less educated, lower income, and non-metro areas had the higher rates of mastectomy $(28.2 \%, 28.6 \%, 28.9 \%, 29.8 \%)$ and BCS without RT $(23.3 \%, 24.8 \%, 26.1 \%, 26.4 \%)$.

$\underline{\text { Associations with Type of Treatment }}$

Age \& Health

Compared to women who were age 66-69 years at diagnosis, all other age groups were more likely to be treated with mastectomy and BCS only, compared to BCS plus RT, with women age $\geq 80$ years being the most likely to have mastectomy vs. BCS plus RT (AOR, 2.32; 95\% CI, 2.17, 2.49) and BCS without RT vs. BCS plus RT (AOR, 3.48; 95\% CI, 3.25, 3.74). Women with greater comorbidity were more likely to have mastectomy or BCS without RT vs. BCS plus RT, compared to women with a comorbidity score of "0". In fact, women with the highest comorbidity were $38 \%$ more likely to receive BCS without RT vs. BCS plus RT (AOR, $1.38,95 \% \mathrm{CI}, 1.28,1.47)$. Correspondingly, women with a high number of PCP visits were more likely to receive mastectomy (AOR, 1.05; 95\% CI, 1.00, 1.08) and BCS only (AOR, 1.13; 95\% CI, 1.06, 1.21) vs. BCS plus RT, compared to women with a low PCP visits (Table 3). 


\section{Clinical Prognostics Factors}

Women diagnosed with stage II breast cancer were more than three times as likely to receive mastectomy (AOR, 3.18; 95\% CI, 3.03, 3.34) and 1.29 times more likely (95\% CI, 1.22, 1.36) to receive BCS without RT vs. BCS plus RT, compared to women diagnosed with stage I breast cancer. Those with negative and borderline/unknown ER tumors were more likely to be treated with mastectomy or BCS without RT vs. BCS plus RT. Women with a negative and borderline/unknown PR status were also more likely to receive mastectomy vs. BCS plus RT.

Finally, women with moderately, poorly, and undifferentiated/unknown tumors were more likely to receive mastectomy vs. BCS plus RT, as compared to women with well differentiated tumors. Women with undifferentiated/unknown tumors were also more likely to receive BCS without RT vs. BCS plus RT (AOR, 1.47; 95\% CI, 1.29, 1.66), compared to women with well differentiated tumors.

\section{Oncology Care Resources}

Women, residing in areas with a high density mammography screening centers were less likely to be treated with BS without vs. BCS plus RT (AOR, 0.88; 95\% CI, 0.79, 0.97), than women residing in low density screening areas. Similarly, women residing in areas with a high density of oncology treatment centers (AOR, 0.87; 95\% CI, 0.79, 0.96) were less likely to have mastectomy vs. BCS plus RT, than women residing in low density areas. Compared to women treated by a general surgeon only, women treated by an oncology surgeon only or treated by both types of surgeons were 23\% (AOR, 0.77; 95\% CI, 0.69, 0.86) and 24\% less likely (AOR, 0.76; 95\% CI, 0.72, 0.82) to receive mastectomy vs. BCS plus RT. Likewise, women treated by an oncology surgeon only or treated by both types of surgeons were $47 \%$ less likely (AOR, 0.53; 
95\% CI, 0.47, 0.60) (AOR, 0.53; 95\% CI, 0.50, 0.56) to receive BCS without RT vs. BCS plus RT, than those treated by a general surgeon only. Socio-Demographic Characteristics

Women of other races were $30 \%$ and $27 \%$ more likely to have mastectomy (AOR, 1.30; 95\% CI, 1.20, 1.40) or BCS without RT vs. BCS plus RT (AOR, 1.24; 95\% CI, 1.14, 1.34), compared to women of white race. In contrast, women living in an area where $\geq 15 \%$ of the population was college educated were less likely to receive mastectomy or BCS without RT vs. BCS plus RT, than women living in an area that was $<15 \%$ college educated. Women residing in areas with an average annual income $>\$ 35,000$ were also less likely to receive mastectomy or BCS without RT vs. BCS plus RT, than women residing in areas with an average annual income $\leq \$ 35,000$. Lastly, women residing in metro areas were $30 \%$ and $27 \%$ less likely to receive mastectomy (AOR, 0.70; 95\% CI, 0.65, 0.75) or BCS without RT vs. BCS plus RT (AOR, 0.73; $95 \% \mathrm{CI}, 0.68,0.79)$, compared to women residing in non-metro areas.

\section{Discussion}

Among elderly women diagnosed with invasive early-stage breast cancer in 2003 thru 2009, 55\% received BCS plus RT as their initial loco-regional treatment, while $23 \%$ had mastectomy, and 22\% had BCS without RT. Somewhat different rates were found by DeSantis and colleagues (2014) who examined patterns of treatment for early-stage breast cancer among women of all ages from the National Cancer Data Base. They reported that $50 \%$ of women received BCS plus RT, 36\% had mastectomy, and 9\% had BCS without RT. ${ }^{40}$ An international comparison of treatment for early-stage breast cancer limited to women age $\geq 65$ years, reported that among US women sampled from 1995 to 2005 SEER-Medicare data, 30\% had BCS plus RT, $41 \%$ had mastectomy, and 19\% had BCS without RT. ${ }^{41}$ While these rates of BCS without 
RT are similar to those found by this study, the rates of BCS with RT are lower and mastectomy are higher. This discrepancy in findings is likely due to differences in study design. Whereas, this study looked at initial definitive surgery, Kiderlan and colleagues (2011) classified surgery as mastectomy if a mastectomy was recorded at any time following BCS. The higher rates of BCS plus RT and lower rates of mastectomy found by this study suggest that a greater number of older women with early-stage breast cancer prefer BCS plus RT vs. mastectomy as their first choice of surgery, but a substantial number eventually require mastectomy. In concurrence with studies that noted a increase in rates of BCS without RT following the publication of the CALGB C9343 trial study results and subsequent NCCN guideline update, this study also found that from 2004 to 2006 elderly women with early-stage breast cancer were more likely to have BCS only than BCS with RT, but after 2006 women were just as likely to receive BCS without RT as they were to have BCS with RT. ${ }^{22,24,30}$ This increase in BCS without RT from 2004 to 2006 mostly likely reflects a change in oncology practice immediately following the guideline change, but this increasing trend may have reversed after 2006 amidst the ensuing controversy over omission of RT for some elderly women.

$\underline{\text { Age \& Health }}$

In accordance with findings of previous studies, this study found that elderly women with breast cancer had an increased chance of being receiving BCS without RT or mastectomy, compared to BCS plus RT. Lack of RT following BCS among elderly women has been strongly linked to increasing age, irrespective of risk factors. ${ }^{22,42}$ In fact, this study found women who were age 80 years and older to be more than 3 times likely to not receive RT, as women age 6669 years. Increasing age is commonly cited as the main reason for omission of RT among elderly women due to greater comorbidity, intolerance to RT toxicity, adverse effects, and shorter life- 
expectancy. ${ }^{42-46}$ Likewise, elderly women may be more likely to receive mastectomy for the same reasons RT is omitted following BCS, to avoid any adverse effects of RT or possible intolerance to toxicity from RT due to greater comorbidity and a general frailty associated with old age. However, not all experts agree that RT is more toxic or less tolerable among elderly women, than younger women. In fact, several studies have found RT to be well-tolerated among elderly women, and determined that it did not increase the risk of myocardial infarction or cardiovascular disease (CVD). ${ }^{47-50}$

Declining health is a burden commonly experienced by the elderly population, and presents additional challenges for tolerating cancer treatments, particularly RT. Accordingly, this study found that elderly women with 2 or more comorbid conditions were $\sim 40 \%$ more likely to not receive RT following BCS and $14 \%$ more likely to be treated with mastectomy. The association between women with greater comorbidity being more likely to have mastectomy or BCS without RT, may explain why women with a high frequency of PCP visits were more likely to be treated with mastectomy or BCS without RT. However, over $20 \%$ of women with a comorbidity score $=0$ did not receive RT following BCS and $23 \%$ were treated with mastectomy, suggesting that increased comorbidity does not always predict type of treatment. This may reflect a preference for mastectomy, due to a desire to avoid necessary RT.

\section{$\underline{\text { Clinical Prognostic Factors }}$}

Women diagnosed with stage II breast cancer were $30 \%$ more likely to not receive RT, compared to women with stage I breast cancer, a finding that may also be explained by older age. Declines in routine mammography screening are commonly observed among elderly women, particularly the oldest of the old. A decline in mammography screening among elderly women occurs due to decreased perceived risk of breast cancer, lack of physician 
recommendation, competing health demands, life-expectancy and potential for more harm than benefit from screening, as indicated by the latest United States Preventive Services Task Force (USPSTF) recommendation on mammography screening for women age $>74$ years. ${ }^{51-56}$ This decrease in screening can, in turn, result in a later stage diagnosis, at which point they are already less likely to receive RT following BCS due to older age. Similarly, women diagnosed at stage II vs. I, were over three times more likely to undergo mastectomy. While the likelihood of mastectomy intuitively increases with increasing stage, it may be that older women are even more likely to have mastectomy at a later stage when BCS plus RT is an option, in order to avoid the toxic and adverse effects of RT and the burden of multiple treatments with RT.

Other clinical prognostic factors were associated with receipt of mastectomy in the expected direction, such that women with negative and borderline/unknown ER and PR tumors statuses, and less differentiated tumor grades had an increased likelihood for mastectomy. ${ }^{8,31}$ These clinical characteristics are associated with poorer prognosis among women with breast cancer and are often more challenging to treat with BCS plus RT. ${ }^{18}$

\section{Oncology Care Resources}

Women who were treated by an oncology surgeon or an oncology surgeon and a general surgeon, opposed to a general surgeon only, were $26 \%$ to $47 \%$ less likely to be treated with mastectomy or BCS without RT, compared to BCS with RT. Surgeon characteristics, such as gender, country of training, year or training, and case-load have be linked to type surgery received for early-stage breast cancer. ${ }^{19,33}$ Evidence suggest that surgeon specialty is also associated with more advanced methods of breast cancer treatment. ${ }^{57}$ Being treated by both an oncology and general surgeon may be an indication of case-load sharing and/or division of services within high volume treatment centers, which are commonly associated with better 
treatment practices. ${ }^{58}$ Moreover, those who have an oncology surgeon as a part of the oncology treatment care may have shorter travel distances to access specialty care. In fact, women residing in areas with a high density of mammography screening centers were less likely to have BCS without RT, while women residing in areas with a high density of oncology treatment centers were less likely to have mastectomy, compared to BCS plus RT. These finding reflect the benefit of residing closer to available oncology care resources. Women who have shorter driving distances to treatment centers may be more likely to choose a treatment such as BCS plus RT, that required multiple trips for repeated treatments. ${ }^{59}$

\section{$\underline{\text { Socio-Demographic Characteristics }}$}

Vulnerable socio-demographic and unfavorable clinical characteristics have long been established as factors associated with health disparities in general and in breast cancer treatment. 19,20,22,23 Accordingly, findings from this study confirmed these associations. Women of nonwhite race, residing in areas that were non-metro, less educated, and low income, were less more likely to be treated with mastectomy or not have RT following BCS. Women with these characteristics may not receive RT following BCS or undergo mastectomy for many of the same reasons. This reasons include a lack of access to specialty care, high quality hospitals, greater travel distances to oncology care, and fewer financial resources, even after accounting for less favorable tumor characteristics observed in African American and Hispanic women. ${ }^{59-62}$ What is more, increased mastectomy rates among various races and ethnicities of non-white women may be associated with country of birth, mastery of the English language, navigation of the healthcare system, and awareness of available treatment options. ${ }^{63}$ 


\section{Limitations \& Strengths}

Several limitations should be kept in mind when interpreting the results of this study. First, many women have multiple surgical claims for lumpectomies, mastectomies, or lumpectomies and mastectomies in various combinations. This study examined only the first surgical claim for purposes of continuity and capturing the initial intended primary treatment. This method does not capture the total number of mastectomies and may overestimate the number of lumpectomies without radiation, due to initial treatment failure. While this study examined the relationships between type of treatment and a range of covariates, it was unable to measure how changing attitudes toward mastectomy were associated with type of primary treatment. Moreover, completion of radiation therapy was not assessed, only the initiation of therapy. Additionally, several of socio-demographic characteristics examined were aggregate census level measures, rather than individual level measures. Finally, the SEER individuals within the SEER database have been found to be of higher income, and higher concentrations of racial and ethnic minorities, as compared to the U.S. population. ${ }^{64}$

Despite these limitations, this study holds several strengths that impart credibility to its findings. To the authors' best knowledge, this is the first study to examine the initial choice of loco-regional treatment for early-stage breast cancer among elderly women. This study examined treatment patterns during times before and after a change in treatment guideline recommendations, pertaining to necessity of RT among a subset of women in this population. Concern has been raised whether this guideline change influenced receipt of RT among women whom RT would have been recommended. A wide range of covariates were examined in relation to type of treatment that included socio-demographic, access, health, clinical, and physician characteristics. Despite containing slightly greater proportions of affluent and minority 
individuals, SEER is widely accepted as representative of the U.S. population, making these results generalizable to the greater population of elderly women with invasive early-stage breast cancer insured by Medicare.

\section{Significance \& Conclusions}

In conclusion, more than half of older women with early-stage breast cancer have BCS plus RT as their initial loco-regional treatment, while fewer than one-fourth have BCS without RT. However, a substantial number may eventually require mastectomy due to treatment failure. Women of increasing age, comorbidity, and stage were the most likely to have mastectomy or BCS without RT, possibly reflecting a reluctance to aggressively treat older women with breast cancer, thereby increasing their risk of recurrence and mortality. Current study findings also highlight the importance of access to oncology care resources on receipt of initial type of treatment. These findings suggest that many elderly women are being undertreated for invasive early-stage breast cancer, and therefore at increased risk for breast cancer mortality and recurrence. Aggressive treatment, including adjuvant therapy should be recommended to all elderly women with early-stage breast who are healthy enough and choose to do so. Some women may require additional patient support resources such as patient education and navigation to ensure equity of care. 


\section{References}

1. Fisher B, Redmond C, Poisson R, Margolese R, Wolmark N, Wickerham L., et al. Eight-year results of a randomized clinical trial comparing total mastectomy and lumpectomy with or without irradiation in the treatment of breast cancer. N Engl J Med. 1989; 320(13): $822-828$.

2. Fisher B, Anderson S, Bryant J, Margolese R, Deutsch M, Fisher E, et al. Twenty-year follow-up trial comparing total mastectomy, lumpectomy, and lumpectomy plus irradiation for the treatment of invasive breast cancer. N Engl J Med. 2002; 347(16): $1233-241$.

3. Veronesi U, Cascinelli N, Mariani L, Greco M, Saccozzi R, Luini A, et al. Twenty-year follow-up of a randomize study comparing breast-conserving surgery with radical mastectomy for early breast cancer. N Engl J Med. 2002; 347(16): 1227-1232.

4. Litière S, Werutsky G, Fentiman I, Rutgers E, Christiaens M, Limbergen E, et al. Breast conserving therapy versus mastectomy for stage I-II breast cancer: 20 year follow-up of the EORTC 10801 phase 3 randomised trial. Lancet Oncol. 2012; 13(4): 412-19.

5. Simone N, Dan T, Shih J, Smith S, Sciuto L, Lita E, et al. Twenty-five year results of the national cancer institute randomized breast conservation trial. Breast Cancer Res Treat. 2012; 132(1): 197-203.

6. NIH Consensus Conference. Treatment of early-stage breast cancer. JAMA. 1991; 265(3): 391-395.

7. American Cancer Society. Cancer Treatment and Survivorship Facts \& Figures 2014-2015. Atlanta: American Cancer Society; 2014.

8. Dragun A, Huang B, Tucker T, Spanos W. Increasing mastectomy rates among all age groups for early stage breast cancer: a 10-year study of surgical choice. Breast J. 2012; 18(4): 318-325.

9. Mahmood U, Hanlon A, Koshy M, Buras R, Chumsri S, Tkaczuk K, et al. Increasing national mastectomy rates for the treatment of early stage breast cancer. Ann Surg Oncol. 2013; 20(5): 1436-1443.

10. Wilke L, Czechura T, Wang C, et al. Repeat surgery after breast conservation for the treatment of stage 0 to II breast carcinoma: a report from the National Cancer Data Base, 2004-2010. JAMA Surg. 2014; 149(12): 1296-1305.

11. McCahill L, Single R, Aiello Bowles E, et al. Variability in reexcision following breast conservation surgery. JAMA. 2012; 307(5): 467-475. 
12. McGuire K, Santillan A, Kaur P, Meade T, Parbhoo J, Mathias M, et al. Are mastectomies on the rise? A 13-year trend analysis of the selection of mastectomy versus breast conservation therapy in 5865 patients. Ann Surg Oncol. 2009; 16(10): 2682-2690.

13. Boscoe F, Johnson C, Henry K, Goldberg D, Shahabi K, Elkin E, et al. Geographic proximity to treatment for early stage breast cancer and likelihood of mastectomy. Breast. 2011; 20(4): 324-328.

14. Goyal S, Chandwani S, Haffty B, Demissie K. Effect of travel distance and time to radiotherapy on likelihood of receiving mastectomy. Ann Surg Oncol. 2014; Sept 23: Epub ahead of print.

15. Bradley C, Given C, Baser O, Gardiner J. Influence of surgical and treatment choices on the cost of breast cancer care. Eur J Health Econ. 2003; 4(2): 96-101.

16. Jacobs L, Kelley K, Rosson G, Detrani M, Chang D. Disparities in urban and rural mastectomy populations : the effects of patient- and county-level factors on likelihood of receipt of mastectomy. Ann Surg Oncol. 2008; 15(10): 2644-2652.

17. Schroen A, Brenin D, Kelly M, Knaus W, Slingluff C. Impact of patient distance to radiation therapy on mastectomy use in early-stage breast cancer patients. J Clin Oncol. 2005; 23(28): 7074-7080.

18. Dragun A, Huang B, Tucker T, Spanos W. Disparities in the application of adjuvant radiotherapy after breast-conserving surgery for early stage breast cancer: impact on overall survival. Cancer. 2011; 117(12): 2590-2598.

19. Hershman D, Buono D, McBride R, Tsai W, Joseph K, Grann V, et al. Surgeon characteristics and receipt of adjuvant radiotherapy in women with breast cancer. $\mathrm{J}$ Natl Cancer Inst. 2008; 100(3): 199-206.

20. Martinez S, Shah D, Tseng W, Canter R, Bold R. Rural-urban disparities in use of postlumpectomy radiation. Med Oncol. 2012; 29(5): 3250-3257.

21. Jagsi R, Abrahamse P, Morrow M, Hawley S, Griggs J, Graff J, et al. Patterns and correlates of adjuvant radiotherapy receipt after lumpectomy and after mastectomy for breast cancer. J Clin Oncol. 2010; 28(14): 2396-2403.

22. Tuttle T, Jarosek S, Habermann E, Yee D, Yuan J, Virnig B. Omission of radiation therapy after breast-conserving surgery in the United States. Cancer. 2012; 118(8): 2004-2013.

23. Showalter S, Grover S, Sharma S, Lin L, Czerniecki B. Factors influencing surgical and adjuvant therapy in stage I breast cancer: a SEER 18 database analysis. Ann Surg Oncol. 2013; 20(4): 1287-1294. 
24. Luu C, Goldstein L, Goldner B, Schoellhammer HF, Chen SL. Trends in radiotherapy after breast-conserving surgery in elderly patients with early-stage breast cancer. Ann Surg Oncol. 2013; 20(10): 3266-3273.

25. Nagar H, Yan W, Christos P, Chao KS, Nori D, Ravi A. Older patients with early-stage breast cancer: adjuvant radiation therapy and predictive factors for cancer-related death. Am J Clin Oncol. 2014; Oct. 20 Epub ahead of print.

26. Hughes K, Schnaper L, Bellon J, et al. Lumpectomy plus tamoxifen with or without irradiation in women age 70 years or older with early breast cancer: long-term follow-up of CALGB 9343. J Clin Oncol. 2013; 31(19): 2382-2387.

27. Hughes K, Schnaper L, Berry D, Cirrincione C, McCormick B, Shank B, et al. Lumpectomy plus tamoxifen with or without irradiation in women 70 years of age or older with early breast cancer. N Engl J Med. 2004; 351(10): 971-977.

28. Carlson R, Allred D, Anderson B, Burstein H, Carter W, Edge S, et al. Breast cancer. Clinical practice guidelines in oncology. J Natl Compr Canc Netw. 2009; 7(2):122-192.

29. Palta M, Palta P, Bhavsar N, Horton J, Blitzblau R. The use of adjuvant radiotherapy in elderly patients with early-stage breast cancer: changes in practice patterns after publication of Cancer and Leukemia Group B 9343. Cancer. 2015; 121(2): 188-193.

30. Rutter C, Lester-Coll N, Mancini B, et al. The evolving role of adjuvant radiotherapy for elderly women with early-stage breast cancer. Cancer. 2015; Mar 24. doi: 10.1002/cncr.29377. [Epub ahead of print].

31. Habermann E, Abbott A, Parsons H, Virnig B, Al-Refaie W, Tuttle T. Are mastectomy rates really increasing in the United States? J Clin Oncol. 2010; 28(21): 3437-3441.

32. McCormick B, Ottesen R, Hughes M, et al. Impact of guideline changes on use or omission of radiation in the elderly with early breast cancer: practice patterns at National Comprehensive Cancer Network institutions. J Am Coll Surg. 2014; 219(4): 796-802.

33. Feigelson H, James T, Single R, Onitilo A, Aiello Bowles E, Barney T, et al. Factors associated with the frequency of initial total mastectomy: results of a multi-institutional study. J Am Coll Surg. 2013; 216(5): 966-975.

34. Smith G, Xu Y, Shih Y, et al. Breast-Conserving Surgery in Older Patients with Invasive Breast Cancer: Current Patterns of Treatment Across the United States. J Am Coll Surg. 2009; 209(4):425-433.e2.

35. Engels E, Pfeiffer R, Ricker W, Wheeler W, Parsons R, Warren J. Use of Surveillance, Epidemiology, and End Results-Medicare data to conduct case-control studies of cancer among the US elderly. Am J Epidemiol. 2011; 174(7): 860-870. 
36. Surveillance epidemiology and end results. SEER-Medicare: About the Data Files. Available at: http://appliedresearch.cancer.gov/seermedicare/aboutdata/. Last accessed on Oct 29, 2014.

37. U.S. Health Resources and Services Administration. Bureau of Health Professions. Area Resource File, 2009-2010 Release Rockville, MD : U.S. Department of Health and Human Services. Fairfax, VA : Quality Resource Systems, Inc. 2010; Codebook: HE001(2010).

38. Klabunde C, Potosky A, Legler J, Warren J. Development of a comorbidity index using physician claims data. J Clin Epidemiol. 2000; 53(12): 1258-1267.

39. Charlson M, Pompei P, Ales K, MacKenzie C. A new method of classifying prognostic comorbidity in longitudinal studies: development and validation. J Chronic Dis. 1987; 40(5): 373-383.

40. DeSantis C, Lin C, Mariotto A, et al. Cancer treatment and survivorship statistics, 2014. CA Cancer J Clin. 2014; 64(4): 252-271.

41. Kiderlen M, Bastiaannet E, Walsh P, et al. Surgical treatment of early stage breast cancer in elderly: an international comparison. Breast Cancer Res Treat. 2012; 132(2): 675-682.

42. Soulos P, Yu J, Roberts K, Raldow A, Herrin J, Long J, et al. Assessing the impact of a cooperative group trial on breast cancer care in the Medicare population. J Clin Oncol. 2012; 30(14):1601-1607.

43. Schonberg M, Marcantonio E, Li D, Silliman R, Ngo L, McCarthy E. Breast cancer among the oldest old: tumor characteristics, treatment choices, and survival. J Clin Oncol. 2010; 28(12): 2038-2045.

44. Yarnold J, Ashton A, Bliss J, Homewood J, Harper C, Hanson J, et al. Fractionation sensitivity and dose response of late adverse effects in the breast after radiotherapy for early breast cancer: long-term results of a randomised trial. Radiother Oncol. 2005; 75(1):9-17.

45. Harris E, Correa C, Hwang W, Liao J, Litt H, Ferrari V, et al. Late cardiac mortality and morbidity in early-stage breast cancer patients after breast-conservation treatment. J Clin Oncol. 2006; 24(25): 4100-4106.

46. Landoni V, Giordano C, Marsella A, Saracino B, Petrongari M, Ferraro A, et al. Evidence from a breast cancer hypofractionated schedule: late skin toxicity assessed by ultrasound. J Exp Clin Cancer Res. 2013; 32(80).

47. Haque R, Prout M, Geiger A, Kamineni A, Thwin S, Avila C, et al. Comorbidities and cardiovascular disease risk in older breast cancer survivors. Am J Manag Care. 2014; 20(1): 86-92. 
48. Doyle J, Neugut A, Jacobson J, Wang J, McBride R, Grann A, et al. Radiation therapy, cardiac risk factors, and cardiac toxicity in early-stage breast cancer patients. Int $\mathrm{J}$ Radiat Oncol Biol Phys. 2007; 68(1): 82-93.

49. Plataniotis G, Theofanopoulou M, Sotiriadou K, Kyrgias G. Hypofractionated radiotherapy for breast cancer patients treated by breast-conserving surgery: short-term morbidity and preliminary results. Breast Cancer. 2010; 17(1): 42-47.

50. Meattini I, Cecchini S, Muntoni C, Scotti V, De Luca Cardillo C, Mangoni M, et al. Cutaneous and cardiac toxicity of concurrent trastuzumab and adjuvant breast radiotherapy: a single institution series. Med Oncol. 2014; 891(4).

51. Schonberg M, McCarthy E, York M, Davis R, Marcantonio E. Factors influencing elderly women's mammography screening decisions: implications for counseling. BMC Geriatr. 2007; 7:26.

52. Walter L, Schonberg M. Screening mammography in older women: a review. JAMA. 2014; 311(13):1336-1347.

53. Haas J, Kaplan C, Des Jarlais G, Gildengoin V, Pérez-Stable E, Kerlikowske K. Perceived risk of breast cancer among women at average and increased risk. J Womens Health (Larchmt). 2005; 14(9):845-851.

54. Feinstein A, Soulos P, Long J, Herrin J, Roberts K, Yu J, et al. Variation in Receipt of Radiation Therapy after Breast-Conserving Surgery: Assessing the Impact of Physicians and Geographic Regions. Med Care. 2013; 51(4): 330-338.

55. Schonberg M, Breslau E, McCarthy E. Targeting of mammography screening according to life expectancy in women aged 75 and older. J Am Geriatr Soc. 2013; 61(3):388-395.

56. Preventive Services Task Force. Screening for breast cancer: U.S. Preventive Services Task Force recommendation statement. Ann Intern Med. 2009; 151(10): 716-726.

57. Yen T, Laud P, Sparapani R, Nattinger A. Surgeon specialization and use of sentinel lymph node biopsy for breast cancer. JAMA Surg. 2014; 149(2):185-192.

58. Kimmick G, Camacho F, Mackley H, Kern T, Yao N, Mathews S, et al. Individual, Area, and Provider Characteristics Associated With Care Received for Stages I to III Breast Cancer in a Multistate Region of Appalachia. J Oncol Pract. 2014; Sep 16. pii:

JOP.2014.001397. [Epub ahead of print].

59. Wheeler S, Kuo T, Durham D, Frizzelle B, Reeder-Hayes K, Meyer A. Effects of distance to care and rural or urban residence on receipt of radiation therapy among North Carolina Medicare enrollees with breast cancer. N C Med J. 2014; 75(4): 239-246. 
60. Keating N, Kouri E, He Y, Weeks J, Winer E. Racial differences in definitive breast cancer therapy in older women are they explained by the hospitals where patients undergo surgery? Med Care. 2009; 47(7): 765-773.

61. Freedman R, He Y, Winer E, Keating N. Trends in racial and age disparities in definitive local therapy of early-stage breast cancer. J Clin Oncol. 2009; 27(5): 713-719.

62. Dookeran K, Silva A, Warnecke R, Rauscher G. Race/ethnicity and disparities in mastectomy practice in the breast cancer care in Chicago study. Ann Surg Oncol. Epub ahead of print Aug 19, 2014. DOI 10.1245/s10434-014-3945-6.

63. Gomez S, Press D, Lichtensztajn D, Keegan T, Shema S, Le G, et al. Patient, hospital, and neighborhood factors associated with treatment of early-stage breast cancer among Asian American women in California. Cancer Epidemiol Biomarkers Prev. 2012; 21(5): 821-834.

64. Warren J, Klabunde C, Schrag D, Bach P, Riley G. Overview of the SEER-Medicare data: content, research applications, and generalizability to the United States elderly population. Med Care. 2002; 40(8 Suppl):IV,3-18. 
Table 1

Characteristics of Elderly Women with Early-Stage Breast Cancer

SEER -Medicare, 2003-2009

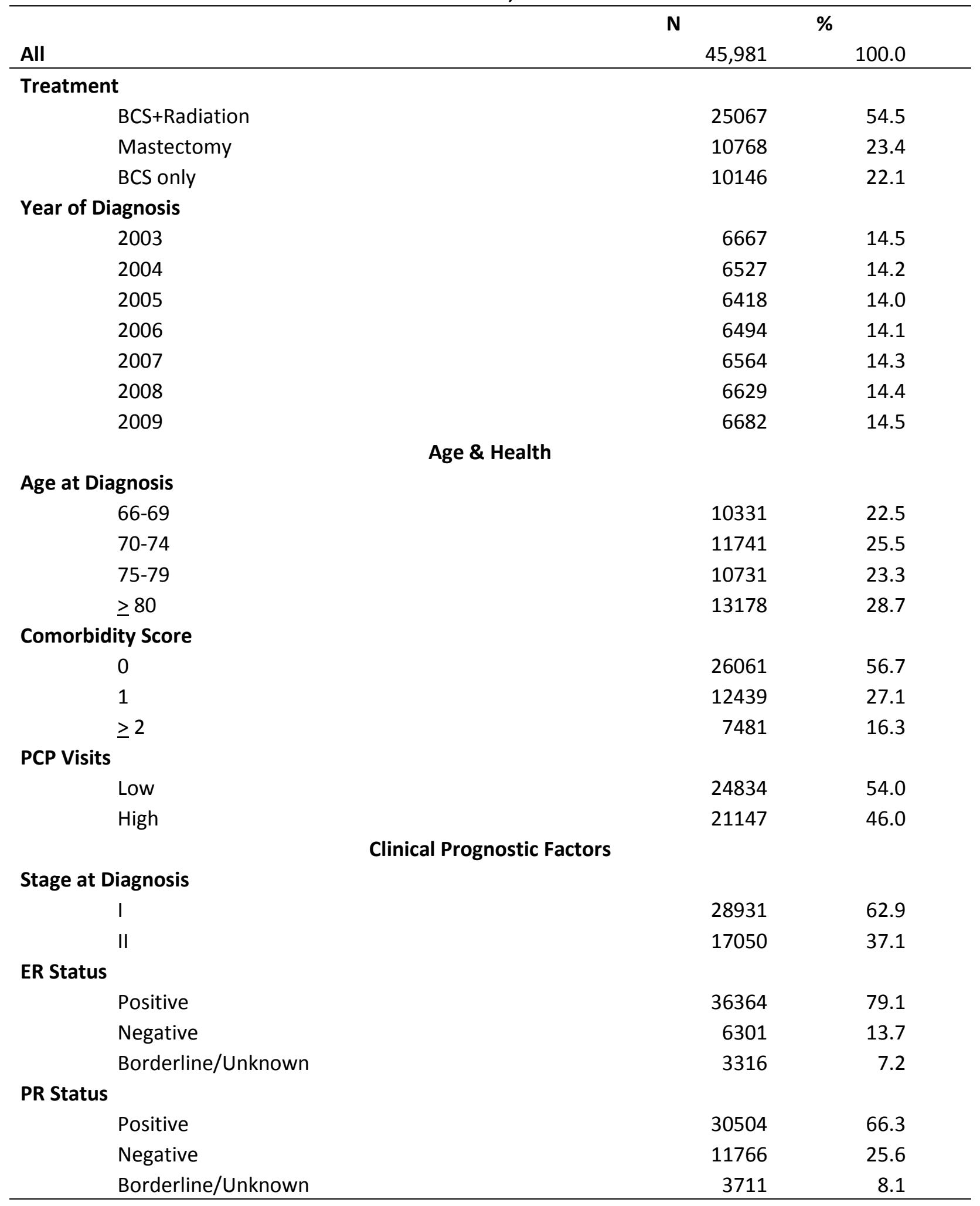

$\mathrm{BCS}=$ breast-conserving surgery 
Table 1

Characteristics of Elderly Women with Early-Stage Breast Cancer

SEER -Medicare, 2003-2009

\begin{tabular}{|c|c|c|}
\hline & $\mathbf{N}$ & $\%$ \\
\hline All & 45,981 & 100.0 \\
\hline \multicolumn{3}{|l|}{ Tumor Grade } \\
\hline Well Differentiated & 12118 & 26.4 \\
\hline Moderately Differentiated & 20046 & 43.6 \\
\hline Poorly Differentiated & 10831 & 23.6 \\
\hline Undifferentiated/Unknown & 2986 & 6.5 \\
\hline \multicolumn{3}{|c|}{ Oncology Care Resources } \\
\hline \multicolumn{3}{|l|}{ Mammography Screening Centers } \\
\hline Low & 23789 & 51.7 \\
\hline High & 22192 & 48.3 \\
\hline \multicolumn{3}{|l|}{ Oncology Treatment Centers } \\
\hline Low & 25761 & 56.0 \\
\hline High & 20220 & 44.0 \\
\hline \multicolumn{3}{|l|}{ Type of Surgeon Seen } \\
\hline General Only & 8555 & 18.6 \\
\hline Oncology Only & 2644 & 5.8 \\
\hline Both & 34782 & 75.6 \\
\hline \multicolumn{3}{|c|}{ Socio-Demographic Characteristics } \\
\hline \multicolumn{3}{|l|}{ Race } \\
\hline White & 23441 & 89.4 \\
\hline Other & 4891 & 10.6 \\
\hline \multicolumn{3}{|l|}{ Education } \\
\hline$<15 \%$ college degree & 13557 & 29.5 \\
\hline$\geq 15 \%$ college degree & 32424 & 70.5 \\
\hline \multicolumn{3}{|l|}{ Annual Income } \\
\hline$\leq \$ 35,000$ & 10853 & 23.6 \\
\hline$>\$ 35,000$ & 35128 & 76.4 \\
\hline \multicolumn{3}{|l|}{ Metro Status } \\
\hline Non-metro & 7686 & 16.7 \\
\hline Metro & 38295 & 83.3 \\
\hline
\end{tabular}

$\mathrm{BCS}=$ breast-conserving surgery 
Table 2

Comparison of Initial Loco-Regional Treatment Among Elderly Women with Early-Stage Breast Cancer SEER-Medicare, 2003-2009

\begin{tabular}{|c|c|c|c|c|c|c|c|}
\hline \multirow[b]{3}{*}{ All } & \multicolumn{2}{|c|}{ BCS+Radiation } & \multicolumn{2}{|c|}{ Mastectomy } & \multicolumn{2}{|c|}{ BCS Only } & \multirow{3}{*}{$p$-value } \\
\hline & $\mathbf{N}$ & $\%$ & $\mathbf{N}$ & $\%$ & $\mathbf{N}$ & $\%$ & \\
\hline & 25067 & 54.5 & 10768 & 23.4 & 10146 & 22.1 & \\
\hline Year of Diagnosis ${ }^{b}$ & & & & & & & $<0.001 * * *$ \\
\hline 2003 & 3523 & 52.8 & 1656 & 24.8 & 1488 & 22.3 & \\
\hline 2004 & 3503 & 53.7 & 1437 & 22.0 & 1587 & 24.3 & \\
\hline 2005 & 3389 & 52.8 & 1462 & 22.8 & 1567 & 24.4 & \\
\hline 2006 & 3572 & 55.0 & 1419 & 21.9 & 1503 & 23.1 & \\
\hline 2007 & 3637 & 55.4 & 1560 & 23.8 & 1367 & 20.8 & \\
\hline 2008 & 3676 & 55.5 & 1610 & 24.3 & 1343 & 20.3 & \\
\hline 2009 & 3767 & 56.4 & 1624 & 24.3 & 1291 & 19.3 & \\
\hline \multicolumn{8}{|c|}{ Age \& Health } \\
\hline Age at Diagnosis $^{c}$ & & & & & & & $<0.001 * * *$ \\
\hline $66-69$ & 6714 & 65.0 & 2067 & 20.0 & 1550 & 15.0 & \\
\hline $70-74$ & 7266 & 61.9 & 2524 & 21.5 & 1951 & 16.6 & \\
\hline $75-79$ & 6035 & 56.2 & 2525 & 23.5 & 2171 & 20.2 & \\
\hline$\geq 80$ & 5052 & 38.3 & 3652 & 27.7 & 4474 & 34.0 & \\
\hline Comorbidity Score $^{c}$ & & & & & & & $<0.001 * * *$ \\
\hline 0 & 14986 & 57.5 & 5892 & 22.6 & 5183 & 19.9 & \\
\hline 1 & 6504 & 52.3 & 2959 & 23.8 & 2976 & 23.9 & \\
\hline$\geq 2$ & 3577 & 47.8 & 1917 & 25.6 & 1987 & 26.6 & \\
\hline PCP Visits ${ }^{a}$ & & & & & & & $<0.001 * * *$ \\
\hline Low & 14157 & 57.0 & 5685 & 22.9 & 4992 & 20.1 & \\
\hline High & 10910 & 51.6 & 5083 & 24.0 & 5154 & 24.4 & \\
\hline \multicolumn{8}{|c|}{ Clinical Prognostic Factors } \\
\hline Stage at Diagnosis ${ }^{a}$ & & & & & & & $<0.001 * * *$ \\
\hline 1 & 17759 & 61.4 & 4517 & 15.6 & 6655 & 23.0 & \\
\hline ॥ & 7308 & 42.9 & 6251 & 36.7 & 3491 & 20.5 & \\
\hline ER Status ${ }^{a}$ & & & & & & & $<0.001 * * *$ \\
\hline Positive & 20599 & 56.7 & 7866 & 21.6 & 7899 & 21.7 & \\
\hline Negative & 3158 & 50.1 & 1912 & 30.3 & 1231 & 19.5 & \\
\hline Borderline/Unknown & 1310 & 39.5 & 990 & 29.9 & 1016 & 30.6 & \\
\hline PR Status $^{a}$ & & & & & & & $<0.001 * * *$ \\
\hline Positive & 17405 & 57.1 & 6461 & 21.2 & 6638 & 21.8 & \\
\hline Negative & 6148 & 52.3 & 3221 & 27.4 & 2397 & 20.4 & \\
\hline Borderline/Unknown & 1514 & 40.8 & 1086 & 29.3 & 1111 & 29.9 & \\
\hline
\end{tabular}

$\mathrm{a}=\mathrm{cmh}$ chi square test of general association

$\mathrm{b}=\mathrm{cmh}$ chi square test of location shift using table scores

$\mathrm{c}=\mathrm{cmh}$ chi square test of location shift using modified ridit scores

$\mathrm{BCS}=$ breast conserving surgery

* $p \leq 0.05 ; * * p \leq 0.01 ; * * * p \leq 0.001$ 
Table 2

Comparison of Initial Loco-Regional Treatment Among Elderly Women with Early-Stage Breast Cancer SEER-Medicare, 2003-2009

\begin{tabular}{|c|c|c|c|c|c|c|c|}
\hline \multirow[b]{3}{*}{ All } & \multicolumn{2}{|c|}{ BCS+Radiation } & \multicolumn{2}{|c|}{ Mastectomy } & \multicolumn{2}{|c|}{ BCS Only } & \multirow{3}{*}{$p$-value } \\
\hline & $\mathbf{N}$ & $\%$ & $\mathbf{N}$ & $\%$ & $\mathbf{N}$ & $\%$ & \\
\hline & 25067 & 54.5 & 10768 & 23.4 & 10146 & 22.1 & \\
\hline Well Differentiated & 7128 & 58.8 & 2197 & 18.1 & 2793 & 23.1 & \\
\hline Moderately Differentiated & 11129 & 55.5 & 4606 & 23.0 & 4311 & 21.5 & \\
\hline Poorly Differentiated & 5414 & 50.0 & 3267 & 30.2 & 2150 & 19.9 & \\
\hline Undifferentiated/Unknown & 1396 & 46.8 & 698 & 23.4 & 892 & 29.9 & \\
\hline \multicolumn{8}{|c|}{ Oncology Care Resources } \\
\hline Mammography Screening Cent & & & & & & & $<0.001 * * *$ \\
\hline Low & 12276 & 51.6 & 6018 & 25.3 & 5495 & 23.1 & \\
\hline High & 12791 & 57.6 & 4750 & 21.4 & 4651 & 21.0 & \\
\hline Oncology Treatment Centers ${ }^{a}$ & & & & & & & $<0.001 * * *$ \\
\hline Low & 13392 & 52.0 & 6492 & 25.2 & 5877 & 22.8 & \\
\hline High & 11675 & 57.7 & 4276 & 21.2 & 4269 & 21.1 & \\
\hline Type of Surgeon Seen ${ }^{a}$ & & & & & & & $<0.001 * * *$ \\
\hline General Only & 3636 & 42.9 & 2031 & 23.7 & 2888 & 33.8 & \\
\hline Oncology Only & 1536 & 59.0 & 607 & 23.0 & 501 & 19.0 & \\
\hline Both & 19895 & 56.2 & 8130 & 23.4 & 6757 & 19.4 & \\
\hline \multicolumn{8}{|c|}{ Socio-Demographic Characteristics } \\
\hline Race $^{a}$ & & & & & & & $<0.001 * * *$ \\
\hline White & 22696 & 55.2 & 9389 & 22.9 & 9005 & 21.9 & \\
\hline Other & 2371 & 48.5 & 1379 & 28.2 & 1141 & 23.3 & \\
\hline Education $^{a}$ & & & & & & & $<0.001 * * *$ \\
\hline$<15 \%$ college degree & 6318 & 46.6 & 3874 & 28.6 & 3365 & 24.8 & \\
\hline$\geq 15 \%$ college degree & 18749 & 57.8 & 6894 & 21.3 & 6781 & 20.9 & \\
\hline Annual Income ${ }^{a}$ & & & & & & & $<0.001 * * *$ \\
\hline$\leq \$ 35,000$ & 4878 & 45.0 & 3141 & 28.9 & 2834 & 26.1 & \\
\hline$>\$ 35,000$ & 20189 & 57.5 & 7627 & 21.7 & 7312 & 20.8 & \\
\hline Metro Status $^{a}$ & & & & & & & $<0.001 * * *$ \\
\hline Non-metro & 3367 & 43.8 & 2289 & 29.8 & 2030 & 26.4 & \\
\hline Metro & 21700 & 56.7 & 8479 & 22.1 & 8116 & 21.2 & \\
\hline
\end{tabular}

$\mathrm{a}=\mathrm{cmh}$ chi square test of general association

$\mathrm{b}=\mathrm{cmh}$ chi square test of location shift using table scores

$\mathrm{c}=\mathrm{cmh}$ chi square test of location shift using modified ridit scores

$\mathrm{BCS}=$ breast conserving surgery

* $\mathrm{p} \leq 0.05 ;{ }^{* *} \mathrm{p} \leq 0.01 ; * * * \mathrm{p} \leq 0.001$ 
Table 3

Associations with Initial Loco-Regional Treatment Among Elderly Women with Early-Stage Breast Cancer SEER-Medicare, 2003-2009

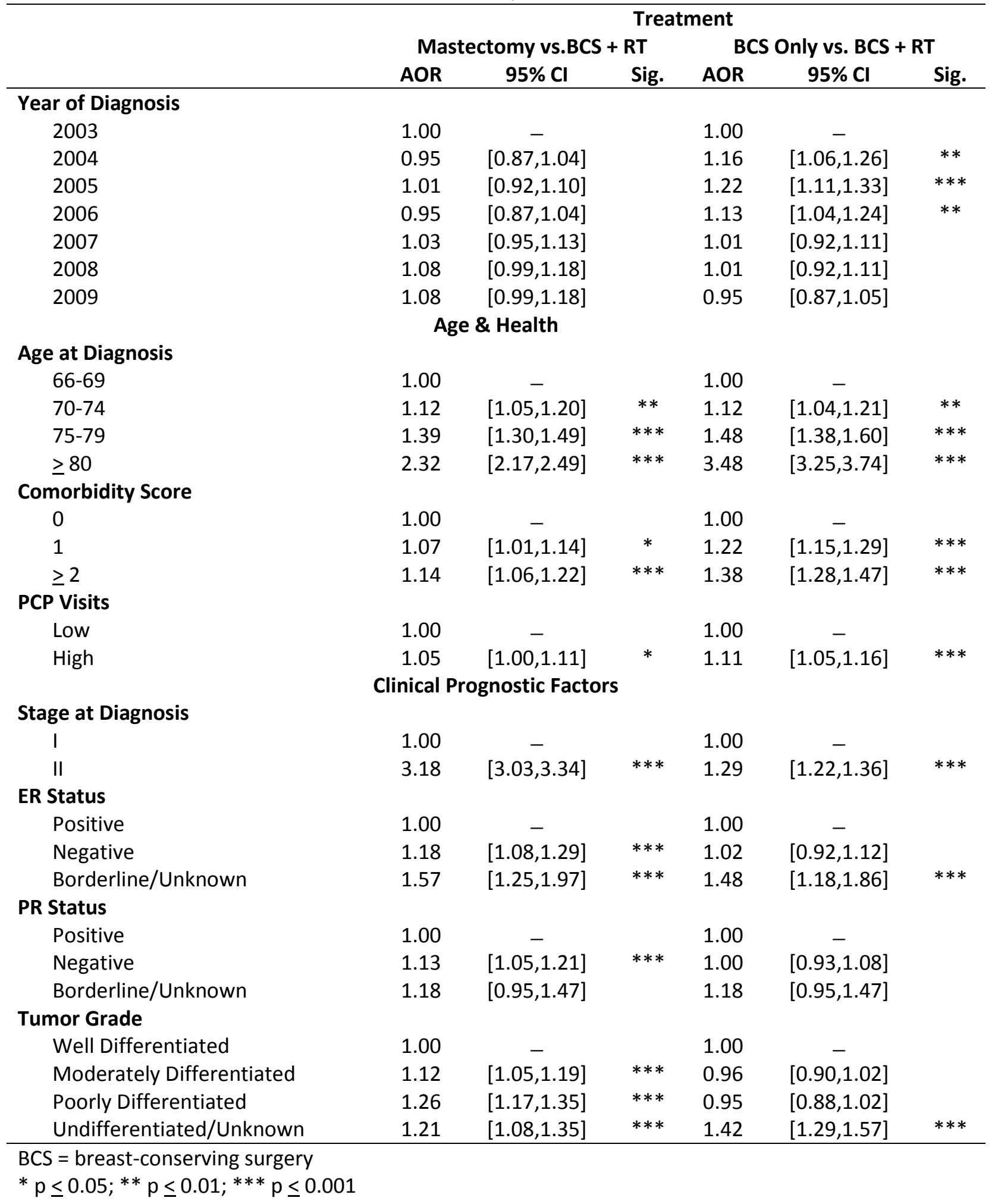


Table 3

Associations with Initial Loco-Regional Treatment Among Elderly Women with Early-Stage Breast Cancer SEER-Medicare, 2003-2009

\section{Treatment}

Mastectomy vs. BCS + RT BCS Only vs. BCS + RT

$\begin{array}{llllll}\text { AOR } & 95 \% \mathrm{Cl} & \text { Sig. } & \text { AOR } & 95 \% \mathrm{Cl} & \text { Sig. }\end{array}$

\section{Oncology Care Resources}

\section{Mammography Screening Centers}

Low

High

Oncology Treatment Centers

Low

High

Type of Surgeon Seen

General Only

Oncology Only

Both

Race

White

Other

Education

$<15 \%$ college degree

$\geq 15 \%$ college degree

Annual Income

$\leq \$ 35,000$

$>\$ 35,000$

Metro Status

Non-metro

Metro

$\mathrm{BCS}=$ breast-conserving surgery

$* p \leq 0.05 ; * * p \leq 0.01 ; * * * p \leq 0.001$
1.00

0.98

$[0.89,1.08]$

1.00

0.87

$[0.79,0.96]$

$* * \quad 1.00$
1.05

$[0.95,1.16]$

$\begin{array}{lccccc}1.00 & - & & 1.00 & - & \\ 0.77 & {[0.69,0.86]} & * * * & 0.53 & {[0.47,0.60]} & * * * \\ 0.76 & {[0.72,0.82]} & * * * & 0.53 & {[0.50,0.56]} & * * *\end{array}$

$\begin{array}{lc}1.00 & - \\ 0.88 & {[0.79,0.97]}\end{array} * *$

\section{Socio-Demographic Characteristics}

1.00

1.30

$[1.20,1.40]$

$\begin{array}{rr} & 1.00 \\ * * * \quad 1.24\end{array}$

$[1.14,1.34]$

1.00

0.75

$[0.70,0.79]$

*** $\quad 0.82$

$[0.77,0.87]$

1.00

0.87

$[0.82,0.93]$

$\begin{array}{rr} & 1.00 \\ * * * \quad 0.84\end{array}$

$[0.78,0.89]$

1.00

0.70

1.00

0.73

$[0.68,0.79]$

$* * * \quad 0.73$

[0.68,0.79] $\quad * * *$


CHAPTER 3 


\title{
CHAPTER 3
}

"Determinants for Receipt of Guideline-Concordant Care among Elderly Women with Stage I-III Breast Cancer in a SEER-Medicare Population"

\author{
Traci LeMasters ${ }^{1}$ \\ S. Suresh Madhavan, M.B.A, Ph.D. Chair ${ }^{1}$ \\ Usha Sambamoorthi Ph.D. ${ }^{1}$ \\ Hannah Hazard M.D. ${ }^{2}$ \\ Kimberly M Kelly Ph.D. 1,3 \\ Dustin Long Ph.D. ${ }^{4}$
}
1 Department of Pharmaceutical Systems and Policy, School of Pharmacy, West Virginia University
2 Department of Medical Education, School of Medicine, West Virginia University 3 Mary Babb Randolph Cancer Center, West Virginia University
${ }^{4}$ Department of Biostatistics, School of Public Health, West Virginia University




\section{Abstract}

Objectives: Evidenced-based guidelines for the treatment of breast cancer have been put forth by agencies such as the National Comprehensive Cancer Network (NCCN). Yet, even with specific guidelines, gaps in the receipt of appropriate care exist, particularly among the aged population. This study examined factors associated with receipt of guideline-concordant care (GCC) vs. guideline-discordant care (GDC) and receipt of distinct treatments and testing among elderly U.S. women with breast cancer.

Methods: A sample of 27,883 women age $\geq 66$ with pathologically staged I, II, and III breast cancer diagnosed from 2004 to 2009 were selected from the SEER-Medicare linked dataset. Algorithms that considered clinical cancer characteristics and the appropriate course of care as per guidelines vs. the actual care received, were used to determine receipt of GCC (yes vs. no). Logistic regression models were used to examine the relationship between GCC and independent variables that included of age, health, clinical prognostic factors, oncology care resources, and socio-demographic characteristics. Age was dichotomized to estimate the likelihood for receipt of specific tests and treatments between younger (age 66-69) and older (age $\geq 70$ ) women.

Results: Less than $34 \%$ of elderly women received guideline appropriate care. Women of nonwhite race (AOR, $0.81 ; 95 \% \mathrm{CI}, 0.73,0.89$ ), increasing age ( $\geq 80$ vs. 66-69) (AOR, $0.19 ; 95 \%$ CI, 0.17, 0.21), increasing comorbidity ( $\geq 2$ vs. 0) (AOR, 0.71; 95\% CI, 0.65, 0.78), and increasing stage (II vs. I) (AOR, 0.46; 95\% CI, 0.40, 0.54) were less likely to receive GCC. Women with node negative cancer, larger tumors, poorly differentiated tumors, negative and borderline/unknown tumor receptors were also less likely to receive appropriate care. Yet, the likelihood of appropriate care was increased for women who were treated by an oncology surgeon (AOR, 1.84; 95\% CI, 1.67, 2.03) or both a general and oncology surgeon vs. general 
surgeon only (AOR, 1.47; 95\% CI, 1.26, 1.72). Compared to women age 66-69 years, those age $\geq 70$ years were more likely to have mastectomy (AOR, 1.31; 95\% CI, 1.23, 1.39) or have any definitive surgery (AOR, 2.51; 95\% CI, 1.76, 3.58), but less likely to have GCC (AOR, 0.51; 95\% CI, 0.48, 0.54), estrogen-receptor status testing (AOR, 0.80; 95\% CI, 0.70, 0.92), progesterone-receptor status testing (AOR, 0.78; 95\% CI, 0.68, 0.89), breast-conserving surgery (AOR, 0.76; 95\% CI, 0.71, 0.81), radiation therapy (AOR, 0.63; 95\% CI, 0.60, 0.67), chemotherapy (AOR, 0.30; 95\%CI, 0.28, 0.32), and appropriate time to chemotherapy (AOR, $0.78 ; 95 \%$ CI, 0.68, 0.89).

Conclusions: Irrespective of guidelines, younger, less complex patients with greater resources are more likely to receive GCC. Better efforts should be made for all elderly women with breast cancer who are healthy enough to receive the aggressive standard of care. 


\section{Introduction}

Breast cancer is the most commonly diagnosed and prevalent cancer among women. As of 2012, over 2.9 million women living with breast cancer in the U.S., and in 2015, an estimated 231,840 incident cases and 40,730 deaths with be attributable to invasive breast cancer (IBC). ${ }^{1,2}$ However, with early detection from routine screening and appropriate treatment according to recommended guidelines, prognosis is relatively good with an $89 \%$ overall 5-year survival rate. ${ }^{2}$ Based on the results of clinical trials, evidenced-based recommended guidelines for the treatment of non-metastatic breast cancer have been put forth by the National Comprehensive Cancer Network (NCCN) to direct the course of breast cancer care and treatment according to cancer and patient characteristics. ${ }^{3}$ Tumor size, spread of cancer to lymph nodes and the number of nodes affected and tumor hormone receptor status determine the type of primary surgery (breast conserving surgery or mastectomy) that a patient is eligible for, and if radiation therapy (RT), chemotherapy, and/or adjuvant hormone therapy (AHT) is recommended. Surgery choice will also determine recommendation for receipt of adjuvant treatments. Moreover, all women with IBC should have their lymph nodes tested for spread of disease and biopsies of breast tissue should be tested to establish whether the tumor is estrogen receptor (ER) positive, progesterone receptor (PR) positive, and/or HER2/neu positive or negative. Additional quality measures for breast cancer treatment jointly published by the American Society of Clinical Oncology (ASCO) and the NCCN suggest that AHT should begin within the first year of diagnosis, RT should begin within the first year of diagnosis, and chemotherapy should begin within 120 days of diagnosis. 4

Despite the existence of evidence-based treatment guidelines, many women with breast cancer still do not receive appropriate, or guideline-concordant care (GCC). As with other health 
disparities, access to specialty care and vulnerable socio-demographic characteristics such as being of non-white race, publicly insured or uninsured, residing in areas of low income, low education, and/or locations, having greater driving distances to treatment centers, and even surgeon characteristics have been associated with guideline-discordant care (GDC) for breast cancer. ${ }^{5-11}$ In addition to these factors, poor clinical characteristics including unknown receptor status and tumor grade have also been linked with GDC. ${ }^{6,9,11}$ The most commonly reported causes for GDC is the omission of lymph node testing, RT following breast-conserving surgery (BCS), RT following mastectomy, chemotherapy, and adjuvant hormone therapy. ${ }^{5,6,8-12}$ An unknown tumor receptor status may also be an indication that the test for receptor status was not conducted. ${ }^{13}$

In addition to these associations, older age and its relationship with increased comorbidity and frailty, and thereby a possible intolerance and/or toxicity to RT or chemotherapy have been strongly associated with GDC among elderly women with breast cancer. ${ }^{5,6,8-11,14-17}$ However, the belief that RT and/or chemotherapy is less tolerable or more toxic in older women compared to younger women, is not universal. In fact, many studies have shown RT and chemotherapy to be well-tolerated and safe among the elderly. ${ }^{18-21}$ Guidelines do not make recommendations for or against chemotherapy in women age $>70$ years, but suggest chemotherapy be given on a case-by-case basis, considering comorbidity. ${ }^{22}$ Moreover, NCCN treatment guidelines make an exception for the omission of RT following BCS among a specific group of women meeting the following requirements: age $\geq 70$ years, tumors $\leq 2 \mathrm{~cm}$, node negative, estrogen-receptor positive, and receiving adjuvant hormone therapy. ${ }^{23}$ This change to recommended treatment guidelines was made after the 2004 publication of the Cancer and Leukemia Group B (CALGB) C9343 trial study results that demonstrated similar 5-year survival 
between women with these clinical characteristics who received BCS plus RT or BCS alone. Since then, rates of RT following BCS have decreased, regardless of clinical factors or the increased risk for breast cancer mortality and recurrence among elderly women not receiving RT after BCS. ${ }^{24-26}$ This trend raises the questions as to what other recommended care are they not receiving and to how often do elderly women with breast cancer receive GCC?

\section{Statement of the Problem}

Few studies have comprehensively studied receipt of GCC among women with breast cancer. One study by Kimmick and colleagues (2014) comprehensively examined GCC in relation to comorbidity among women of all ages diagnosed with stage 0 thru stage III, but their sample was limited to women diagnosed in 2004 from seven cancer registries. ${ }^{27}$ Other studies investigating GCC have done so in piecemeal fashion, either looking at GCC among women with early-stage breast cancer only, specific racial/ethnic populations, limited loco-regional treatment, systematic treatment, using small study samples, older data, and/or allowed for the omission of RT or chemotherapy among women age $\geq 70$ years from GCC criteria. ${ }^{5,13,27-31}$ To address gaps in the literature and build upon existing knowledge of GCC among elderly women, this study will comprehensively examine receipt of hormone receptor and lymph node status testing, definitive surgery, RT, chemotherapy, and timeliness of chemotherapy among women age $\geq 66$ years diagnosed with stage I, II, III breast cancer from 2004 to 2009 using a large, nationally representative database. In order to determine to what degree older populations of women with breast cancer receive care according to recommended treatment guidelines as they would otherwise be applied to younger age groups, this study included treatment with RT and chemotherapy for women age $\geq 70$ years with clinical indications in the definition of GCC. 
Bearing these thoughts in mind, the purpose of this study was to determine to what degree elderly women with breast cancer receive GCC, factors associated with receipt of GCC, and how likely women age $\geq 70$ vs. age $66-69$ years are to receive specific tests and treatments.

\section{Methods}

$\underline{\text { Data }}$

The SEER-Medicare database was created in a collaborative effort by the National Cancer Institute (NCI) and Centers for Medicare and Medicaid Services (CMS) to combine clinical information gathered by the Surveillance Epidemiology and End Results cancer registry program with Medicare insurance claims. The SEER program collects basic socio-demographic, clinical, and vital status information on incident cancer cases from multiple reporting sources. This information is found the Patient Entitlement and Diagnosis Summary File (PEDSF). SEER data used for this study consisted of 17 population-based registries, representing $26 \%$ of the U.S. population. An algorithm based on name, Social Security number, sex, and date of birth successfully matched $94 \%$ SEER cases to their Medicare claims beginning from the time of eligibility till death. ${ }^{32}$ This study used Medicare claims providing information regarding beneficiary enrollment (Denominator File), hospital inpatient claims (Med-PAR file), hospital outpatient claim (Outpatient file), physician office visits ( $\mathrm{NCH}$ file), and durable medical equipment (DME file). ${ }^{33}$ Additionally, county and state of diagnosis were used to identify the area-level density of mammography screening and oncology centers from the U.S. Department of Health and Human Resource's 2009 Area Resource File (ARF). ${ }^{34}$ $\underline{\text { Study Population }}$

This retrospective cohort study selected American Joint Commission on Cancer (AJCC) staged I, II, and III incident cases of breast cancer recorded in SEER during years 2004 to 2009, 
among female age-eligible Medicare beneficiaries who were continuously enrolled in Medicare Part A and B fee-for-service programs 1 year before and after diagnosis, and were therefore age $\geq 66$ years at the time of diagnosis. Women were excluded if they were enrolled in a managed care program at any time during year before to the year after diagnosis, diagnosed at the time of death or upon autopsy, died within the first year of diagnosis, had end-stage renal disease, did not have primary surgical treatment, or if surgeon specialty or tumor size was missing. The final sample size was $n=27,883$ elderly women.

\section{$\underline{\text { Measures }}$}

Dependent Variables

The primary outcome of interest was receipt of GCC vs. GDC. This was determined by comparing the correct course of care according to tumor size and nodal status of each women as per NCCN evidence-based clinical practice guidelines, to the actual care received (Table 1.) Care was also determined to be guideline-concordant if chemotherapy was initiated within 120 days of diagnosis, when chemotherapy was indicated, as per ASCO/NCCN quality measures, and if lymph node invasion, and hormone receptor statuses were tested. Although ASCO/NCCN quality measures indicate RT should be initiated within 1 year of diagnosis, this was not considered in the determination of GCC, as all claims used for this study were made within 1 year after diagnosis. For this study purpose, being of age $\geq 70$ years was not considered a determining factor for GCC vs. GDC when RT or chemotherapy was indicated, in order to determine the extent that younger and older women receive differential care.

The secondary outcome of interest was the receipt of individual treatments and tests. These included lymph node testing (yes or no), ER status testing (yes or no), PR status testing (yes or no), had any primary surgery (BCS or mastectomy) (yes or no), had BCS (yes or no), had 
mastectomy (yes or no), had RT (yes or no), had chemotherapy (yes or no), and chemotherapy was initiated within 120 day of diagnosis (yes, no, or not applicable). For women who had BCS and mastectomy, both types of surgery were included in analysis, with totals from both category adding to greater than $100 \%$. Hormone receptor status testing was assessed using the status given by the ER status and PR status variable in the Patient Entitlement and Diagnosis Summary File (PEDSF) file. If the status was listed as "positive", "negative", or "borderline", then the receptor status was determined to have been tested. If the status was listed as "unknown", then it was considered as not having been tested. Lymph node testing was defined by the claims codes for axillary lymph node dissection (ALND) or sentinel lymph node (SNB) biopsy. Surgical, RT, and chemotherapy treatments were determined using International Classification of Diseases, 9th Revision (ICD-9) diagnostic and procedure, Healthcare Common Procedure Coding System (HCPCS) and Current Procedural Terminology (CPT) codes found in Medicare Provider and Analysis Review (MEDPAR), Outpatient, and Physician/Supplier Data File data files. See Appendix 1.

Independent Variables

Independent variables examined in this study encompassed year of diagnosis, age, health, clinical prognostic factors, specific tests and treatments, oncology care resources, and sociodemographic characteristics. Age at diagnosis $(66-69,70-74,75-79, \geq 80)$ and health were considered together since declining health associated with increasing age is the reason given by NCCN guidelines for case-by-case use of chemotherapy in women older than 70 years, and are often cited as associations with undertreatment among elderly women in retrospective studies. Age was also dichotomized (66-69 or $\geq 70)$ to test for differences between younger and older elderly women. Health was measured by calculating patient comorbidity by applying an 
algorithm to Medicare claims that identifies a comorbid condition as the presence of at least one in-patient or two out-patient claims using ICD-9 diagnoses codes for each co-morbid condition. Overall comorbidity was then classified using the Klabunde adaption of the Charlson comorbidity index score $(0,1, \geq 2) .{ }^{35,36}$ Frequency of primary care provider (PCP) visits was also used as a proxy for health status, with a higher number of visits considered an indication of poorer health. An index of PCP visit intensity was identified by counting of number of unique PCP claim dates the year before diagnosis in the $\mathrm{NCH}$ file. $\mathrm{PCP}$ visits were categorized by lower and upper 50th percent median cutoff (low, high). Clinical prognostic factors examined were stage at diagnosis, tumor size $(<1 \mathrm{~cm},<2 \mathrm{~cm}, 2-5 \mathrm{~cm},>5 \mathrm{~cm})$, lymph node status (positive or negative), ER tumor status (positive, negative, borderline/unknown), PR tumor status (positive, negative, borderline/unknown), and tumor grade (well differentiated, moderately differentiated, poorly differentiated, undifferentiated/unknown). Stage diagnosis was categorized using the pathologically staged AJCC Stage Group 6th edition (I, II, III). Individual tests and treatments examined were ER tumor status testing (yes or no), PR tumor status testing (yes or no), lymph node status testing (yes or no), receipt of any definitive surgery (yes or no), mastectomy (yes or no), BCS (yes or no), RT (yes or no), chemotherapy (yes or no), and initiation of chemotherapy within 120 days of diagnosis (yes or no). Measures of oncology care resources were the density of area-level mammography screening centers and oncology treatment centers relative to each woman's location of residence. This area-level density was measured using data from the ARF and was categorized by lower and upper 50th percent median cutoff (low, high). Surgeon specialty was assessed using provider specialty claims codes 02,49 (general) and 83, 90, 91, 98 (oncology) from the $\mathrm{NCH}$ file to determine the type of surgeon seen (general only, oncology only, both). Socio-demographic characteristics examined were race (white, other), education 
(measured by the 2000 Census tract survey of percent of persons age $\geq 25$ with at least 4 years college education) $(<15 \%, \geq 15 \%)$, annual income (measured by the 2000 Census tract survey of median income by census) $(\leq \$ 35,000,>\$ 35,000)$, and metro status (non-metro, metro). $\underline{\text { Analysis }}$

Descriptive analysis was conducted for all study variables. Mantel-Haenszel chi-square tests of location shift using modified ridit scores or table scores and Mantel-Haenszel chi-square tests of general association (depending on whether the independent measure was dichotomous, nominal, or ordinal) were used to test for significant group differences between GCC vs. GDC and independent measures, with significance set at $P \leq .05$. Binomial logistic regression models were used to estimate the adjusted odds of receiving GCC vs. GDC for each of the independent variables described above and for receipt of specific tests and treatments for women age $\geq 70$ years vs. age 66-69 years old, controlling for the independent measures. Parameter estimates calculated in the regression models are presented as adjusted odds ratios (AOR) with their corresponding $95 \%$ confidence intervals (CI). All analysis were conducted using SAS version 9.4 software (SAS Institute Inc., Cary, NC).

\section{Results}

\section{$\underline{\text { Population Characteristics }}$}

Among this sample of elderly female fee-for-service Medicare beneficiaries diagnosed with pathologically staged breast cancer in 2004 thru 2009, the majority were age 70-74 years at diagnosis $(27.1 \%)$, of white race $(88.2 \%)$, lived in metro areas $(83.3 \%)$, areas where the population was $\geq 15 \%$ college educated $(69.5 \%)$ and had an annual income $>\$ 35,000(75.6 \%)$. The majority had a comorbidity score $=0$ (55.7\%), were diagnosed at Stage I (56.4\%), had their were lymph node negative (73.1\%), had BCS (74.8\%), had RT (61.1\%), did not have 
chemotherapy (74.9\%), saw both a general and oncology surgeon (79.6\%), and did not receive $\operatorname{GCC}(66.5 \%)$.

\section{Group Differences by Receipt of GCC}

All group differences were significant at the $P \leq .001$ level, with the exception of density of mammography screening centers $(P=.219)$ and density of oncology centers $(P=.083)$.

Receipt of GCC increased from $31.9 \%$ in 2004 to $35.2 \%$ in 2009. Yet, receipt of GCC decreased with increasing age ( $45.8 \%$ for those age $66-69$ years vs. $17.2 \%$ for those age $\geq 80$ years) and increasing comorbidity $(35.3 \%$ for those with comorbidity score $=0$ vs. $27.9 \%$ with comorbidity score $\geq 2)$. Women diagnosed as Stage I had the highest rates of GCC (39.7\%), whereas those diagnosed at II had the lowest rates of GCC (22.6\%). Additional clinical prognostic factors with lower rates of GCC were positive lymph nodes, larger tumor size, positive and borderline/unknown ER status, positive and borderline/unknown PR status, and undifferentiated/unknown tumor grades. Women who were treated by an oncology surgeon or both an oncology and general surgeon had higher rates of GCC (32.8\% \& 35.1\%, respectively) than women treated by a general surgeon only (25.2\%). In regards to socio-demographic characteristics women of white race vs. non-white, lived in metro areas vs. non-metro, areas where $\geq 15 \%$ of the population was college educated vs. $<15 \%$, or average annual income was $>$ $\$ 35,000$ vs. $\leq \$ 35,000$ had higher rates of GCC.

Associations with Receipt of GCC

Receipt of GCC became less likely with increasing age, such that women age $\geq 80$ years (AOR, 0.19; 95\% CI, 0.17, 0.21) were the least likely to receive $\mathrm{GCC}$, compared to women age 66-69 years. Women with a comorbidity score $\geq 2$ were about $30 \%$ less likely (AOR, 0.71; $95 \%$ $\mathrm{CI}, 0.65,0.78)$ to receive GCC, than women with a score = zero. Compared to women diagnosed 
at Stage I, those diagnosed at Stage II were less than half as likely to receive GCC (AOR, 0.46; 95\% CI, 0.40, 0.54). Likewise, women with larger tumors were less likely to have had GCC, than women with tumors $<1 \mathrm{~cm}$. Elderly women with lymph node negative breast cancer were less than half as likely to have had GCC (AOR, $0.49 ; 95 \% \mathrm{CI}, 0.44,0.56)$, than women who were lymph node positive. However, women who had tumors that were ER and PR negative vs. positive were more likely to receive GCC (AOR, 1.89; 95\% CI, 1.69, 2.11) and (AOR, 1.20; 95\% CI, 1.10, 1.32), respectively. Whereas, those whose tumors were ER and PR borderline/unknown were $96 \%$ and $68 \%$ less likely to receive GCC (AOR, $0.04 ; 95 \%$ CI, 0.02 , 0.07 ) and PR statuses (AOR, 0.32; 95\% CI, 0.22, 0.47), respectively. In contrast, women with moderately and poorly differentiated tumors vs. well-differentiated, were more likely to have had GCC. Women treated by an oncology surgeon or both a general and oncology surgeon were $47 \%$ (AOR, 1.47; 95\% CI, 1.26, 1.72) and 84\% (AOR, 1.84; 95\% CI, 1.67, 2.03), more likely to receive GCC, respectively, than women treated by a general surgeon only. Women of non-white race vs. white were less likely to receive GCC (AOR, $0.81 ; 95 \% \mathrm{CI}, 0.73,0.89$ ), whereas women who resided in areas where $\geq 15 \%$ was college educated vs. $<15 \%$ were more likely to receive GCC.

Associations with Tests \& Treatments among Women Age $>70$ vs. $66-69$ years

Women age $\geq 70$ years were half as likely ( $\mathrm{AOR}, 0.51 ; 95 \% \mathrm{CI}, 0.48,0.54)$ to receive GCC, than women age 66-69 years. Compared to younger women, older women were less likely to have ER status testing (AOR, 0.80; 95\% CI, 0.70, 0.92) and PR status testing (AOR, 0.78; $95 \% \mathrm{CI}, 0.68,0.89)$. However, older women were 2.5 times more likely than younger women to have had any definitive surgery (AOR, $2.51 ; 95 \% \mathrm{CI}, 1.76,3.58$ ). In regards to type of surgery, older women were less likely to have BCS (AOR, 0.76; 95\% CI, 0.71, 0.81), but more likely to 
have mastectomy (AOR, 1.31; 95\% CI, 1.23, 1.39), than younger women. Finally, older women were older women were less likely to have RT (AOR, 0.63; 95\% CI, 0.60, 0.67), chemotherapy (AOR, 0.30; 95\% CI, 0.28, 0.32), or appropriate time to chemotherapy (AOR, 0.78; 95\% CI, $0.68,0.89)$, compared to younger women.

\section{Discussion}

To these authors knowledge, this is the first study to comprehensively examine GCC as treatment guidelines are applied to all women with breast cancer among a large nationally representative sample of elderly women diagnosed at stage I, II, and III, stages for which guidelines are clearly defined. Applying to a strict definition of GCC, this study found that fewer than $34 \%$ of elderly women diagnosed with breast cancer in 2004 thru 2009 received care concordant with NCCN and ASCO recommended treatment guidelines.

\section{$\underline{\text { Age \& Health }}$}

In regards to age, the highest rates of GCC were observed among women age 66-69 years, at $46 \%$ and lowest rates among women age $\geq 80$ years, at $17 \%$. In fact, women age $\geq 70$ years were less than half as likely to receive GCC, than women age 66-69 years. Previous studies

have reported receipt of GCC to range from $35 \%-88 \%$ among women with breast cancer. ${ }^{5,27-}$ 29,37 The variation in these rates of GCC are likely attributable to differences in study design and sample selection, that include varying definitions of GCC (local and systematic treatment, local treatment only, or systematic therapy only), exempting women age $\geq 70$ years from recommended RT or chemotherapy, the inclusion of women age $<65$ years in their study sample, data collected from different years, samples that were limited to earlier stages or included Stage 0, sampled from smaller registries, and/or fewer number of registries. Despite their differences, all studies reported a strong inverse relationship between receipt of GCC and 
age. Increasing age, along with increasing comorbidity and declining functional performance, is commonly cited by oncologists as a primary reason for less aggressive breast cancer treatment. ${ }^{38,39}$ Among elderly women, those age $\geq 70$ are particularly less likely to receive RT after BCS or chemotherapy when indicated, than younger women. In fact, a greater number of women than those meeting guideline exemption from RT, and many women who could benefit from chemotherapy, do not receive these treatments. ${ }^{40-42}$ This study found women age $\geq 70$ years to be as much as $45 \%, 73 \%$, and $34 \%$ less likely to have RT, chemotherapy, or receive chemotherapy within an appropriate time period, respectively, than those 66-69 years of age. Undertreatment of the elderly is a phenomenon observed across different cancers and countries. ${ }^{43-46}$ Although the disparity in aggressive treatment between younger and older elderly breast cancer patients is widely acknowledged, the application of aggressive treatment among older patients continues to be debated. Conflicting findings have been reported regarding the benefits of adjuvant treatments. Ten-year results of the CALGB 9343 trial comparing BCS only vs. BCS plus RT among women age $\geq 70$ years with Stage I, ER positive breast cancer taking tamoxifen showed no differences in breast cancer specific and overall survival, and minimal difference in recurrence (2\% vs. $10 \%) .{ }^{47}$ Similar findings were reported by the more recent PRIME II randomized controlled trial, but with no difference in 5-year overall survival, and little difference in recurrence (1\% vs. 4\%). ${ }^{48}$ However, the National Surgical Adjuvant Breast and Bowel Project (NSABP) B-17 randomized trial demonstrated that women treated with BCS only had significantly higher rates of recurrence after 15 years (19\% vs. 9\%), than women treated with BCS plus RT. ${ }^{49}$ Multiple retrospective database studies support these findings. A recently published study using SEER-Medicare data to compare treatment outcomes among women age 70-79 years with Stage I, ER positive breast cancer treated with BCS only vs. BCS plus RT, 
found that after 10 years, women treated with BCS only ended up having a mastectomy twice as often, as women treated with BCS plus RT. ${ }^{50}$ Further studies comparing these treatment groups using SEER-Medicare and cancer registry data from the British Columbia Cancer Agency also reported an increased risk for breast cancer recurrence and mortality among women treated with BCS only, compared to BCS plus RT. ${ }^{51-54}$ In contrast to study findings of RT outcomes, the survival and disease-free benefits of chemotherapy for women with breast cancer of all ages is generally agreed upon. ${ }^{55-57}$ Despite the demonstrated benefits of chemotherapy and RT, whether these adjuvant therapies should be administered to elderly women as often as younger women with breast cancer when indicated, remains a topic of debate due to their possible harm. Toxic effects and treatment burden from RT and chemotherapy have the potential for adverse effects on health and quality of life. ${ }^{58-61}$ Often, these effects may be amplified in the elderly due to increased comorbidity, impaired organ function, declining functional status, lack of reliable transportation for repeated treatments, or are unjustifiable depending on projected life expectancy. ${ }^{62,63}$ Yet, among elderly women with low comorbidity and good functional status, adjuvant therapy may be well tolerated just and no more toxic, than among younger women. ${ }^{64,65}$ Moreover, many maintain that the harm of potential toxic and adverse side effects of adjuvant therapy, outweigh the potential benefits. ${ }^{60,61}$ Nevertheless, chemotherapy should be initiated within a timely manner as to not compromise survival. ${ }^{66,67}$

In conjunction with increasing age, increasing comorbidity has been strongly associated with less aggressive treatment and GDC among elderly breast cancer patients. ${ }^{37,39,40}$ Patients with increased comorbidity may be less likely to receive GCC for reasons similarly associated with increasing age, that include competing health demands, decreased functional status, and decreased overall survival because of their comorbidity. ${ }^{68,69}$ Despite these concerns, comorbidity 
itself has not been linked with increased toxicity to adjuvant treatment or time to recurrence, and thus, should not prevent GCC when patients are strong and functional enough to endure the demands of adjuvant treatments. ${ }^{70}$

\section{Clinical Prognostic Factors}

Aside from age and health, clinical characteristics were strongly associated with GCC. Women who were diagnosed at later stages, had larger tumors, moderately and poorly tumor grades, borderline/unknown tumors hormone receptors, and were lymph node negative were less likely to receive GCC. This most likely reflects the reluctance of providers to use aggressive treatment in elderly women that would otherwise be recommended for more advanced cancers. It is possible that while women who were lymph node negative did not require chemotherapy, they may have been indicated for RT following BCS, but were less likely to receive it due to older age. In contrast to these associations, women with ER and PR negative tumors were $90 \%$ to $20 \%$ more likely to receive GCC, respectively. A plausible explanation for these findings may lie with the poorer survival outcomes observed among women negative hormone receptors. ${ }^{71,72}$ Therefore, they may be more likely to be aggressively treated with RT and chemotherapy, women with positive hormone receptors, thus making them likely to receive GCC

\section{$\underline{\text { Oncology Care Resources }}$}

Women who were treated by an oncology surgeon only or both a general and oncology surgeon, compared to a general surgeon only, were more likely to receive GCC. This sharing of care may be a sign of high patient volume, a factor that has been associated with increased GCC. Also, the involvement of an oncology surgeon in coordination of care may ensure that lymph node surgery is performed. ${ }^{29}$ 


\section{$\underline{\text { Socio-Demographic Characteristics }}$}

Additional factors associated with receipt of GCC were race and education. Women of non-white race, and those residing in areas of lower education were less likely to receive GCC. The association between vulnerable socio-demographic characteristics and disparities in breast cancer care have been well documented. ${ }^{13,27}$ While the relationship between health disparities and residents of low education areas may be explained by limited resources and access to specialty services, the relationship between race and receipt of GCC is not well understood, especially in the context of uniform Medicare coverage. A recently published study by Freedman and colleagues (2015) may shed light on this subject. ${ }^{73}$ Compared to white women, black and Hispanic women were less likely to be knowledgeable about their breast cancer characteristics, a factor that may prevent them from taking part in the treatment decision process. Similarly, increasing age was also associated with decreased breast cancer knowledge. While it is not realistic to recommend aggressive breast cancer treatment to all elderly patients due to variation in health and functional status, treatment patterns among this population could be improved if emphasis was placed upon dissemination of GCC, unless patients are otherwise unable to do so. In the meantime, patient navigation programs have shown to help direct patients to receipt of GCC and adhere to the course of treatment. ${ }^{74,75}$ Vulnerable populations, including the elderly, may benefit from such patient navigation and education programs to aid in their understanding, decision making process, and ultimately receipt of GCC.

\section{Limitations \& Strengths}

Several limitations should be kept in mind when interpreting the results of this study. This study did not exempt women age $\geq 70$ with tumors $\leq 2 \mathrm{~cm}$, node negative, estrogenreceptor positive, and receiving adjuvant hormone therapy from radiation or women age $>70$ 
years from chemotherapy in determining GCC, and instead examined the application of GCC to all ages to compare treatment differences. This method may have underestimated the number of women receiving GCC due to poor health or functional status. This study did not assess the completion of radiation or chemotherapy, only the initiation of therapy, nor did this study distinguish between the receipt of neoadjuvant or adjuvant chemotherapy. Neoadjuvant chemotherapy is received by some women to shrink larger tumors and provide an opportunity for BCS, after clinical staging, but before pathological staging has been conducted. Thus, this study did not capture how the use of neoadjuvant chemotherapy, and resulting differences in clinical and pathological staging were associated with receipt of GCC or individual tests and treatments. Furthermore, this study did not examine how genetic testing for specific genetic mutations related to breast cancer and subsequent findings were associated with treatment. Also, tumor receptor status was used as a proxy measure of for tumor receptor testing and does not provide any information regarding the initiation of adjuvant hormone therapy when indicated. Moreover, this study was unable to examine receipt of adjuvant hormone therapy. Additionally, several of socio-demographic characteristics examined were aggregate census level measures, rather than individual level measures. Finally, the SEER individuals within the SEER database have been found to be of higher income, and higher concentrations of racial and ethnic minorities, as compared to the U.S. population. ${ }^{76}$

Despite these limitations, this study holds several strengths that impart confidence in its findings. This is one of few studies to investigate GCC among women with breast cancer and possibly the first to do so comprehensively among elderly women using a large, nationally representative database. Pathological staging was used to determine the cancer characteristics of the study sample and algorithms were used to identify receipt of GCC based on the 
recommended course of treatment according to cancer characteristics put forth by the NCCN and ACSO. A variety of tests and treatments were included in this study and examined in relation to younger and older elderly women. Additionally, a wide range of covariates were controlled for that included age, health, clinical prognostic factors, access to oncology care resources, and socio-demographic characteristics. Despite containing slightly greater proportions of affluent and minority individuals, SEER is widely accepted as representative of the U.S. population, making these results generalizable to the greater population of elderly women with invasive early-stage breast cancer insured by Medicare.

\section{Significance \& Conclusions}

In conclusion, rates of GCC among elderly women as determined the current study are much lower than those observed by other studies that were heavily populated by younger women. These findings indicate that older women with breast cancer receive considerably less aggressive treatment than younger women, although they have the highest incidence of breast cancer. Among elderly women, receipt of GCC decreases with increasing age and comorbidity. Yet, about $2 / 3$ s of women with no comorbidity still do not receive GCC, suggesting that age alone or other factors not related to strength and vitality are affecting receipt of GCC. Receipt of GCC also decreases with later stage at diagnosis and worse prognostic clinical factors, circumstances that necessitate more aggressive treatment, but perhaps reflect a reluctance to do so. Women older than 70 years are especially less likely to receive RT and/or chemotherapy. A greater number of elderly women may benefit from receiving GCC than those that actually receive it. In order to maximize the probability of disease-free survival and life-expectancy, patients, family members, and oncology care providers should be encouraged to accept the same 
standard of aggressive breast cancer treatment to older women, as would be applied to younger women. 


\section{References}

1. American Cancer Society. Breast Cancer Facts \& Figures 2013-2014. Atlanta: American Cancer Society, Inc. 2013. Available at:

http://www.cancer.org/acs/groups/content/@ research/documents/document/acspc042725.pdf. Last accessed on Jan 19, 2015.

2. American Cancer Society. Cancer Facts \& Figures 2015. Atlanta: American Cancer Society; 2015. Available at:

http://www.cancer.org/acs/groups/content/@editorial/documents/document/acspc044552.pdf. Last accessed on Jan 19, 2015.

3. Gradishar W, Anderson B, Blair S, Burstein H, Cyr A, Elias A, et al. Breast cancer version 3.2014. J Natl Compr Canc Netw. 2014; 12(4): 542-90.

4. Desch C, McNiff K, Schneider E, Schrag D, McClure J, Lepisto E, et al. American Society of Clinical Oncology/National Comprehensive Cancer Network Quality Measures. J Clin Oncol. 2008; 26(21): 3631-3637.

5. Anderson R, Morris C, Kimmick G, Trentham-Dietz A, Camacho F, Wu X, et al. Patterns of Locoregional Treatment for Nonmetastatic Breast Cancer by Patient and Health System Factors. Cancer. 2014; Nov 4. doi: 10.1002/cncr.29092. [Epub ahead of print].

6. Bhargava A \& Du X. Racial and socioeconomic disparities in adjuvant chemotherapy for older women with lymph node-positive, operable breast cancer. Cancer. 2009; 115(13): 2999-3008.

7. Smith G, Shih Y, Xu Y, Giordano S, Smith B, Perkins G, et al. Racial Disparities in the Use of Radiotherapy After Breast-Conserving Surgery. Cancer. 2010; 116(3): 734-741.

8. Jagsi R, Abrahamse P, Morrow M, Hawley S, Griggs J, Graff J, et al. Patterns and correlates of adjuvant radiotherapy receipt after lumpectomy and after mastectomy for breast cancer. J Clin Oncol. 2010; 28(14): 2396-2403.

9. Hershman D, Buono D, McBride R, Tsai W, Joseph K, Grann V, et al. Surgeon characteristics and receipt of adjuvant radiotherapy in women with breast cancer. J Natl Cancer Inst. 2008; 100(3): 199-206.

10. Halpern M, Chen A, Marlow N, Ward E. Disparities in Receipt of Lymph Node Biopsy Among Early-Stage Female Breast Cancer Patients. Ann Surg Oncol. 2009; 16(3):562570 .

11. Dragun A, Huang B, Tucker T, Spanos W. Disparities in the application of adjuvant radiotherapy after breast-conserving surgery for early stage breast cancer: impact on overall survival. Cancer. 2011; 117(12): 2590-2598. 
12. Nuegut A, Hillyer G, Kushi L, Lamerato L, Nathanson S, Ambrosone C, et al. The Breast Cancer Quality of Care Study (BQUAL): A Multi-Center Study to Determine Causes for Noncompliance with Breast Cancer Adjuvant Therapy. Breast J. 2012; 18(3): 203-213.

13. Freedman R, Virgo K, He Y, Pavluck A, Winer E, Ward E, et al. The Association of Race/Ethnicity, Insurance Status, and Socioeconomic Factors With Breast Cancer Care. 2010; 117(1): 180-189.

14. Sheppard V, Faul L, Luta G, Clapp J, Yung R, Wang J, et al. Frailty and Adherence to Adjuvant Hormonal Therapy in Older Women With Breast Cancer: CALGB Protocol 369901. J Clin Oncol. 2014; 32(22): 2318-2327.

15. Yarnold J, Ashton A, Bliss J, Homewood J, Harper C, Hanson J, et al. Fractionation sensitivity and dose response of late adverse effects in the breast after radiotherapy for early breast cancer: long-term results of a randomised trial. Radiother Oncol. 2005; 75(1):9-17.

16. Landoni V, Giordano C, Marsella A, Saracino B, Petrongari M, Ferraro A, et al. Evidence from a breast cancer hypofractionated schedule: late skin toxicity assessed by ultrasound. J Exp Clin Cancer Res. 2013; 32(80).

17. Reinisch M, Minckwitz G, Harbeck N, Janni W, Kümmel S, Kaufmann M, et al. Side Effects of Standard Adjuvant and Neoadjuvant Chemotherapy Regimens According to Age Groups in Primary Breast Cancer. Breast Care. 2013; 8(1): 60-66.

18. Haque R, Prout M, Geiger A, Kamineni A, Thwin S, Avila C, et al. Comorbidities and cardiovascular disease risk in older breast cancer survivors. Am J Manag Care. 2014; 20(1): 86-92.

19. Doyle J, Neugut A, Jacobson J, Wang J, McBride R, Grann A, et al. Radiation therapy, cardiac risk factors, and cardiac toxicity in early-stage breast cancer patients. Int $\mathrm{J}$ Radiat Oncol Biol Phys. 2007; 68(1): 82-93.

20. Plataniotis G, Theofanopoulou M, Sotiriadou K, Kyrgias G. Hypofractionated radiotherapy for breast cancer patients treated by breast-conserving surgery: short-term morbidity and preliminary results. Breast Cancer. 2010; 17(1): 42-47.

21. Meattini I, Cecchini S, Muntoni C, Scotti V, De Luca Cardillo C, Mangoni M, et al. Cutaneous and cardiac toxicity of concurrent trastuzumab and adjuvant breast radiotherapy: a single institution series. Med Oncol. 2014; 891(4).

22. Carlson R, Allred D, Anderson B, Burstein H, Carter W, Edge S, et al. Breast cancer. Clinical practice guidelines in oncology. J Natl Compr Canc Netw. 2009; 7(2):122-192.

23. Hughes K, Schnaper L, Berry D, Cirrincione C, McCormick B, Shank B, et al. Lumpectomy plus tamoxifen with or without irradiation in women 70 years of age or older with early breast cancer. N Engl J Med. 2004; 351(10): 971-977. 
24. Tuttle T, Jarosek S, Habermann E, Yee D, Yuan J, Virnig B. Omission of radiation therapy after breast-conserving surgery in the United States. A Population-Based Analysis of Clinicopathologic Factors. Cancer. 2012; 118(8): 2004-2013.

25. Luu C, Goldstein L, Goldner B, Schoellhammer HF, Chen SL. Trends in radiotherapy after breast-conserving surgery in elderly patients with early-stage breast cancer. Ann Surg Oncol. 2013; 20(10): 3266-3273.

26. Nagar H, Yan W, Christos P, Chao KS, Nori D, Ravi A. Older patients with early-stage breast cancer: adjuvant radiation therapy and predictive factors for cancer-related death. Am J Clin Oncol. 2014; Oct. 20 Epub ahead of print.

27. Wu X, Lund M, Kimmick G, Richardson L, Sabatino S, Chen V, et al. Influence of race, insurance, socioeconomic status, and hospital type on receipt of guideline-concordant adjuvant systemic therapy for locoregional breast cancers. J Clin Oncol. 2012; 30(2): $142-150$.

28. Guy G, Lipscomb J, Gillespie T, Goodman M, Richardson L, Ward K. Variations in Guideline-Concordant Breast Cancer Adjuvant Therapy in Rural Georgia. Health Serv Res. 2014; Dec 10. doi: 10.1111/1475-6773.12269. [Epub ahead of print].

29. Kimmick G, Camacho F, Mackley H, Kern T, Yao N, Mathews S, et al. Individual, Area, and Provider Characteristics Associated With Care Received for Stages I to III Breast Cancer in a Multistate Region of Appalachia. J Oncol Pract. 2014; Sep 16. pii: JOP.2014.001397. [Epub ahead of print].

30. Buist D, Ichikawa L, Prout M, et al. Receipt of appropriate primary breast cancer therapy and adjuvant therapy are not associated with obesity in older women with access to health care. J Clin Oncol. 2007; 25(23): 3428-3436.

31. Javid S, Varghese T, Morris A, et al. Guideline-concordant cancer care and survival among American Indian/Alaskan Native patients. Cancer. 2014; 120(14): 2183-2190.

32. Engels E, Pfeiffer R, Ricker W, Wheeler W, Parsons R, Warren J. Use of Surveillance, Epidemiology, and End Results-Medicare data to conduct case-control studies of cancer among the US elderly. Am J Epidemiol. 2011; 174(7): 860-870.

32. Surveillance epidemiology and end results. SEER-Medicare: About the Data Files. Available at: http://appliedresearch.cancer.gov/seermedicare/aboutdata/. Last accessed on Oct 29, 2014.

34. U.S. Health Resources and Services Administration. Bureau of Health Professions. Area Resource File, 2009-2010 Release Rockville, MD : U.S. Department of Health and Human Services. Fairfax, VA : Quality Resource Systems, Inc. 2010; Codebook: HE001(2010). 
35. Klabunde C, Potosky A, Legler J, Warren J. Development of a comorbidity index using physician claims data. J Clin Epidemiol. 2000; 53(12): 1258-1267.

36. Charlson M, Pompei P, Ales K, MacKenzie C. A new method of classifying prognostic comorbidity in longitudinal studies: development and validation. J Chronic Dis. 1987; 40(5): 373-383.

37. Kimmick G, Fleming S, Sabatino S, Wu X, Hwang W, Wilson J, et al. Comorbidity Burden and Guideline-Concordant Care for Breast Cancer. J Am Geriatr Soc. 2014; 62(3):482488.

38. Foster J, Salinas G, Mansell S, Willaimson J, Casebeer L. How Does Older Age Influence Oncologists' Cancer Management? The Oncologist. 2010;15(6):584-592.

39. Hurria A, Wong L, Villaluna D, Bhatia S, Chung C, Mortimer J, et al. Role of Age and Health in Treatment Recommendations for Older Adults With Breast Cancer: The Perspective of Oncologists and Primary Care Providers. J Clin Oncol. 2008; 26(33): 5386-5392.

40. McCormick B, Ottesen R, Hughes M, Javid S, Khan S, Mortimer J, et al. Impact of Guideline Changes on Use or Omission of Radiation in the Elderly with Early Breast Cancer: Practice Patterns at National Comprehensive Cancer Network Institutions. J Am Coll Surg. 2014; 219(4):796-802.

41. Mackey R, Chandru Kowdley G. Treatment practices and outcomes of elderly women with breast cancer in a community hospital. Am Surg. 2014; 80(7): 714-719.

42. Wheeler S, Carpenter W, Peppercorn J, Schenck A, Weinberger M, Biddle A. Predictors of timing of adjuvant chemotherapy in older women with hormone receptor-negative, stages II-III breast cancer. Breast Cancer Res Treat. 2012; 131(1): 207-216.

43. Chagpar R, Xing Y, Chiang Y, Feig B, Chang G, You Y, et al. Adherence to Stage-Specific Treatment Guidelines for Patients With Colon Cancer. J Clin Oncol. 2012; 30(9): 972979.

44. Chen R, Carpenter W, Hendrix L, Bainbridge J, Wang A, Nielsen M, et al. Receipt of Guideline-Concordant Treatment in Elderly Prostate Cancer Patients. Int J Radiat Oncol Biol Phys. 2014; 88(2): 332-338.

45. Hancke1 K, Denkinger M, Konig J, Kurzeder C, Wockel A, Herr D, et al. Standard treatment of female patients with breast cancer decreases substantially for women aged 70 years and older: a German clinical cohort study. Ann Oncol. 2010; 21(4): 748-753. 
46. Weggelaar I, Aben K, Warle M, Strobbe L, van Spronsen D. Declined Guideline Adherence in Older Breast Cancer Patients: A Population-Based Study in The Netherlands. Breast J. 2011; 17(3): 239-245.

47. Hughes K, Schnaper L, Bellon J, Cirrincione C, Berry D, McCormick B, et al. Lumpectomy Plus Tamoxifen With or Without Irradiation in Women Age 70 Years or Older With Early Breast Cancer: Long-Term Follow-Up of CALGB 9343. J Clin Oncol. 2013; 31(19): 2382-2387.

48. Kunkler I, Williams L, Jack W, Cameron D, Dixon J. Breast-conserving surgery with or without irradiation in women aged 65 years or older with early breast cancer (PRIME II): a randomised controlled trial. Lancet Oncol. 2015; Jan 27. doi: 10.1016/S14702045(14)71221-5. [Epub ahead of print].

49. Wapnir I, Dignam J, Fisher B, Mamounas E, Anderson S, Julian T, et al. Long-Term Outcomes of Invasive Ipsilateral Breast Tumor Recurrences After Lumpectomy in NSABP B-17 and B-24 Randomized Clinical Trials for DCIS. J Natl Cancer Inst. 2011;103(6): 478-488.

50. Albert J, Pan I, Shih Y, Jiang J, Buchholz T, Giordano S, et al. Effectiveness of Radiation for Prevention of Mastectomy in Older Breast Cancer Patients Treated With Conservative Surgery. Cancer. 2012; 118(19): 4642-4651.

51. Shen X, Anne P, Keith S, Wojcieszynski A, Mishra M, Bar-Ad V, et al. Radiation therapy use and outcomes among older women with ER-positive and ER-negative stage I breast cancer. Am J Clin Oncol. 2014; 37(3): 241-247.

52. Smith B, Haffty B, Buchholz T, Smith G, Galusha D, Bekelman J, et al. Effectiveness of Radiation Therapy in Older Women With Ductal Carcinoma In Situ. J Natl Cancer Inst. 2006; 98(18): 1302-1310.

53. Truong P, Bernstein V, Lesperance M, Speers C, Olivotto I. Radiotherapy omission after breast-conserving surgery is associated with reduced breast cancer-specific survival in elderly women with breast cancer. Am J Surg. 2006; 191(6): 749-755.

54. Sun S, Hollenbeak C, Leung A. Deviation from the Standard of Care for Early Breast Cancer in the Elderly: What are the Consequences? Ann Surg Oncol. 2014; Dec 17: DOI 10.1245/s10434-014-4290-5 [Epub ahead of print].

55. Muss H, Berry D, Cirrincione C, Theodoulou M, Mauer A, Kornblith A, et al. Adjuvant Chemotherapy in Older Women with Early-Stage Breast Cancer. N Engl J Med 2009; 360(20): 2055-2065.

56. Giordano S, Duan Z, Kuo Y, Hortobagyi G, Goodwin J. Use and Outcomes of Adjuvant Chemotherapy in Older Women With Breast Cancer. J Clin Oncol. 2006; 24(18): 27502756. 
57. Elkin E, Hurria A, Mitra N, Schrag D, Panageas K. Adjuvant Chemotherapy and Survival in Older Women With Hormone Receptor-Negative Breast Cancer: Assessing Outcome in a Population-Based, Observational Cohort. J Clin Oncol. 2006; 24(18): 2757-2764.

58. Harris E, Correa C, Hwang W, Liao J, Litt H, Ferrari V, et al. Late cardiac mortality and morbidity in early-stage breast cancer patients after breast-conservation treatment. J Clin Oncol. 2006; 24(25): 4100-4106.

59. Landoni V, Giordano C, Marsella A, Saracino B, Petrongari M, Ferraro A, et al. Evidence from a breast cancer hypofractionated schedule: late skin toxicity assessed by ultrasound. J Exp Clin Cancer Res. 2013; 32(80).

60. Kornblith A, Lan L, Archer L, Partridge A, Kimmick G, Hudis C, et al. Quality of Life of Older Patients With Early-Stage Breast Cancer Receiving Adjuvant Chemotherapy: A Companion Study to Cancer and Leukemia Group B 49907. J Clin Oncol. 2011; 29(8): $1022-1028$.

61. Freedman R, Vaz-Luis I, Barry W, Lii H, Lin N, Winer E, et al. Patterns of chemotherapy, toxicity, and short-term outcomes for older women receiving adjuvant trastuzumab-based therapy. Breast Cancer Res Treat. 2014; 145(2): 491-501.

62. Muss H, Berry D, Cirrincione C, Budman D, Henderson I, Citron M, et al. Toxicity of older and younger patients treated with adjuvant chemotherapy for node-positive breast cancer: the Cancer and Leukemia Group B Experience. J Clin Oncol. 2007; 25(24): 3699-3704.

63. Hurria A, Hurria A, Brogan K, Panageas K, Pearce C, Norton L, et al. Effect of Creatinine Clearance on Patterns of Toxicity in Older Patients Receiving Adjuvant Chemotherapy for Breast Cancer. Drugs Aging. 2005; 22(9): 785-791.

64. Shayne M, Culakova E, Wolff D, Poniewierski M, Dale D, Crawford J, et al. Dose intensity and hematologic toxicity in older breast cancer patients receiving systemic chemotherapy. Cancer. 2009; 115(22): 5319-5328.

65. Garg P, Rana F, Gupta R, Buzaianu E, Guthrie T. Predictors of Toxicity and Toxicity Profile of Adjuvant Chemotherapy in Elderly Breast Cancer Patients. The Breast Journal. 2009; 15(4): 404-408.

66. Downing A, Twelves C, Forman D, Lawrence G, Gilthorpe M. Time to Begin Adjuvant Chemotherapy and Survival in Breast Cancer Patients: A Retrospective Observational Study Using Latent Class Analysis. The Breast Journal. 2014; 20(1): 29-36.

67. Gagliato D, Gonzalez-Angulo A, Lei X, Theriault R, Giordano S, Valero V, et al. Clinical Impact of Delaying Initiation of Adjuvant Chemotherapy in Patients With Breast Cancer. J Clin Oncol. 2014; 32 (8):735-744. 
68. Ahern T, Lash T, Thwin S, Silliman R. Impact of Acquired Comorbidities on All-Cause Mortality Rates Among Older Breast Cancer Survivors. Med Care. 2009; 47(1): 73-79.

69. Sonmez O, Arslan U, Esbah O, Helvaci K, Turker I, Uyeturk U, et al. Effects of comorbidities and functional living activities on survival in geriatric breast cancer patients. Contemp Oncol (Pozn). 2014; 18(3): 204-210.

70. Klepin H, Pitcher B, Ballman K, Kornblith A, Hurria A, Winer E, et al. Comorbidity, Chemotherapy Toxicity, and Outcomes Among Older Women Receiving Adjuvant Chemotherapy for Breast Cancer on a Clinical Trial: CALGB 49907 and CALGB 361004 (Alliance). J Oncol Pract. 2014; 10(5): e285-292.

71. Colzani E, Liljegren A, Johansson A, Adolfsson J, Hellborg H, Hall P, et al. Prognosis of patients with breast cancer: causes of death and effects of time since diagnosis, age, and tumor characteristics. J Clin Oncol. 2011; 29(30): 4014-4021.

72. Yood M, Owusu C, Buist D, Geiger A, Field T, Thwin S, et al. Mortality impact of lessthan-standard therapy in older breast cancer patients. J Am Coll Surg. 2008; 206(1):6675 .

73. Freedman R, Kouri E, West D, Keating N. Racial/Ethnic Disparities in Knowledge About One's Breast Cancer Characteristics. Cancer. 2015; Jan 26. doi: 10.1002/cncr.28977. [Epub ahead of print].

74. Ko N, Darnell J, Calhoun E, Freund K, Wells K, Shapiro C, et al. Can Patient Navigation Improve Receipt of Recommended Breast Cancer Care? Evidence From the National Patient Navigation Research Program. Clin Oncol. 2014; 32(25): 2758-2764.

75. Freund K, Battaglia T, Calhoun E, Darnell J, Dudley D, Fiscella K, et al. Impact of Patient Navigation on Timely Cancer Care: The Patient Navigation Research Program. J Natl Cancer Inst. 2014; 106(6): dju115. doi: 10.1093/jnci/dju115.

76. Warren J, Klabunde C, Schrag D, Bach P, Riley G. Overview of the SEER-Medicare data: content, research applications, and generalizability to the United States elderly population. Med Care. 2002; 40(8 Suppl):IV,3-18. 
Table 1

Characteristics of Elderly Women with Stages I, II, and III Breast Cancer SEER-Medicare, 2004-2009

\begin{tabular}{|c|c|c|}
\hline \multirow[b]{2}{*}{ All } & \multicolumn{2}{|c|}{$\%$} \\
\hline & 27,883 & 100.0 \\
\hline \multicolumn{3}{|l|}{ Year of Diagnosis } \\
\hline 2004 & 3885 & 13.9 \\
\hline 2005 & 3726 & 13.4 \\
\hline 2006 & 3873 & 13.9 \\
\hline 2007 & 4025 & 14.4 \\
\hline 2008 & 6151 & 22.1 \\
\hline 2009 & 6223 & 22.3 \\
\hline \multicolumn{3}{|c|}{ Age \& Health } \\
\hline \multicolumn{3}{|l|}{ Age at Diagnosis } \\
\hline $66-69$ & 6694 & 24.0 \\
\hline $70-74$ & 7551 & 27.1 \\
\hline $75-79$ & 6704 & 24.0 \\
\hline$\geq 80$ & 6934 & 24.9 \\
\hline \multicolumn{3}{|l|}{ Comorbidity Score } \\
\hline 0 & 15520 & 55.7 \\
\hline 1 & 7559 & 27.1 \\
\hline$\geq 2$ & 4804 & 17.2 \\
\hline \multicolumn{3}{|l|}{ PCP Visits } \\
\hline Low & 15354 & 55.1 \\
\hline High & 12529 & 44.9 \\
\hline \multicolumn{3}{|c|}{ Clinical Prognostic Factors } \\
\hline \multicolumn{3}{|l|}{ Stage at Diagnosis } \\
\hline I & 15731 & 56.4 \\
\hline ॥ & 9514 & 34.1 \\
\hline III & 2638 & 9.5 \\
\hline \multicolumn{3}{|l|}{ Tumor Size } \\
\hline$<1 \mathrm{~cm}$ & 8201 & 29.4 \\
\hline$<2 \mathrm{~cm}$ & 10701 & 38.4 \\
\hline $2-5 \mathrm{~cm}$ & 7834 & 28.1 \\
\hline$>5 \mathrm{~cm}$ & 1147 & 4.1 \\
\hline \multicolumn{3}{|l|}{ Lymph Nodes } \\
\hline Positive & 7505 & 26.9 \\
\hline Negative & 20378 & 73.1 \\
\hline \multicolumn{3}{|l|}{ ER Status } \\
\hline Positive & 22298 & 80.0 \\
\hline Negative & 4136 & 14.8 \\
\hline Borderline/Unknown & 1449 & 5.2 \\
\hline \multicolumn{3}{|l|}{ PR Status } \\
\hline Positive & 18762 & 67.3 \\
\hline Negative & 7463 & 26.8 \\
\hline Borderline/Unknown & 1658 & 6.0 \\
\hline
\end{tabular}

$\mathrm{BCS}=$ breast-conserving surgery

$\mathrm{GCC}=$ Guideline-Concordant Care 
Table 1

Characteristics of Elderly Women with Stages I, II, and III Breast Cancer SEER-Medicare, 2004-2009

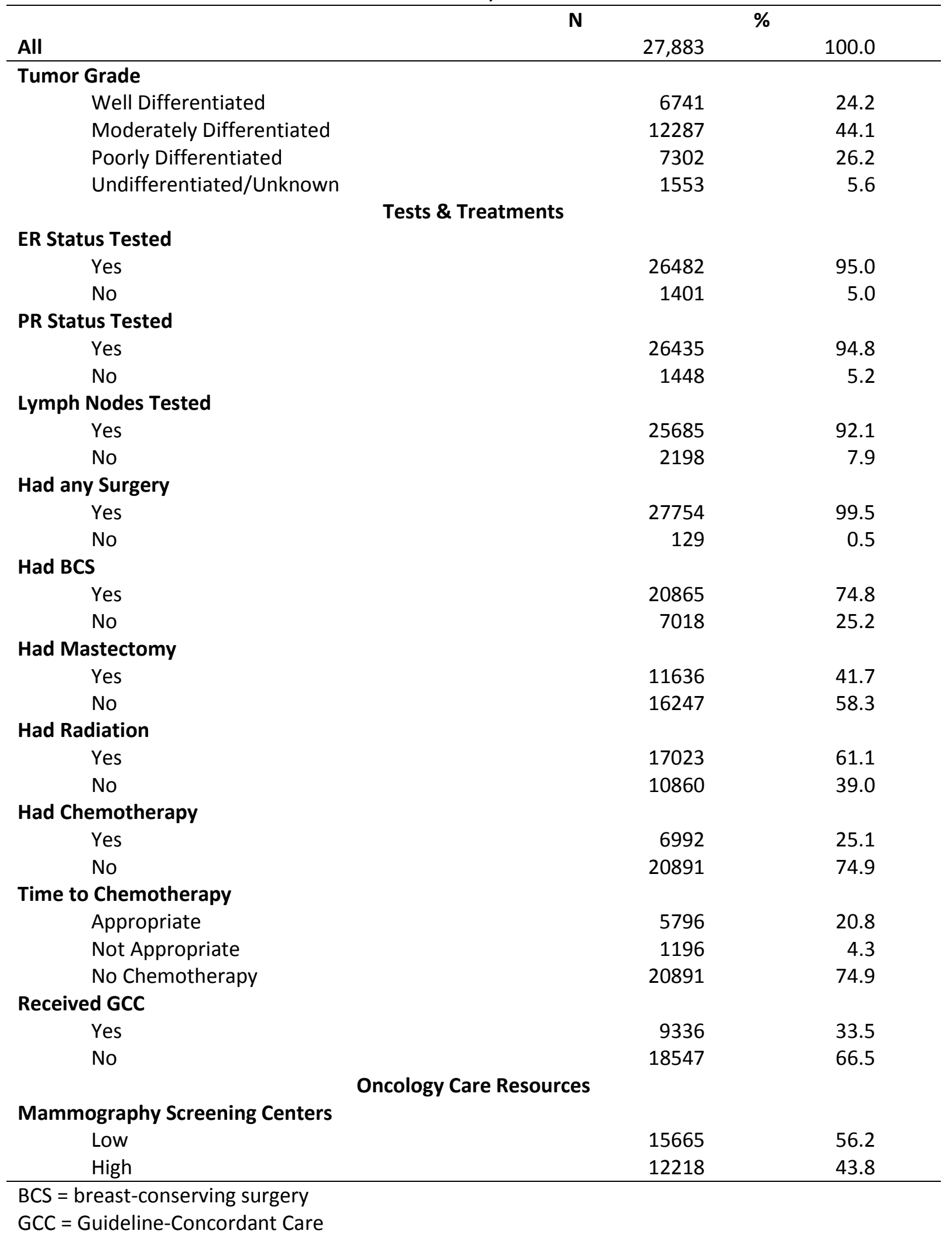


Table 1

Characteristics of Elderly Women with Stages I, II, and III Breast Cancer SEER-Medicare, 2004-2009

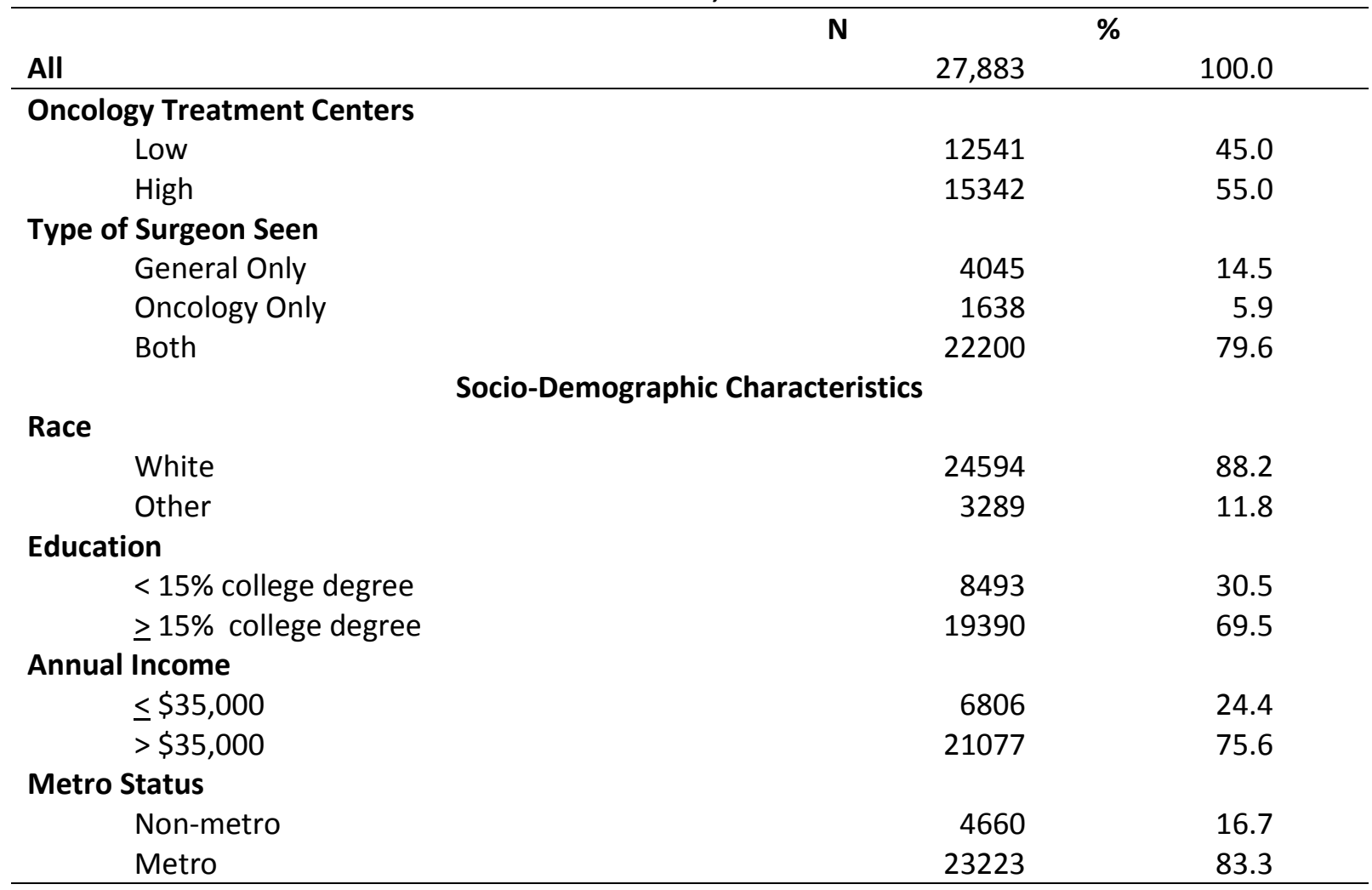

$\mathrm{BCS}=$ breast-conserving surgery

GCC = Guideline-Concordant Care 
Table 2

Comparison of Guideline-Concordant Care among Elderly Women with Stages I, II, and III Breast Cancer

SEER-Medicare, 2004-2009

\begin{tabular}{|c|c|c|c|c|c|}
\hline & \multicolumn{2}{|c|}{ Concordant } & \multicolumn{2}{|c|}{ Discordant } & \multirow{3}{*}{$p$-value } \\
\hline & $\mathbf{N}$ & $\%$ & $\mathbf{N}$ & $\%$ & \\
\hline All & 9,336 & 33.5 & 18,547 & 66.5 & \\
\hline \multicolumn{5}{|l|}{ Year of Diagnosis ${ }^{b}$} & $<0.001 * * *$ \\
\hline 2004 & 1240 & 31.9 & 2645 & 68.1 & \\
\hline 2005 & 1166 & 31.3 & 2560 & 68.7 & \\
\hline 2006 & 1234 & 31.9 & 2639 & 68.1 & \\
\hline 2007 & 1334 & 33.1 & 2691 & 66.9 & \\
\hline 2008 & 2170 & 35.3 & 3981 & 64.7 & \\
\hline 2009 & 2192 & 35.2 & 4031 & 64.8 & \\
\hline \multicolumn{6}{|c|}{ Age \& Health } \\
\hline Age at Diagnosis ${ }^{c}$ & & & & & $<0.001 * * *$ \\
\hline $66-69$ & 3066 & 45.8 & 3628 & 54.2 & \\
\hline $70-74$ & 2966 & 39.3 & 4585 & 60.7 & \\
\hline $75-79$ & 2114 & 31.5 & 4590 & 68.5 & \\
\hline$\geq 80$ & 1190 & 17.2 & 5744 & 82.8 & \\
\hline Comorbidity Score ${ }^{c}$ & & & & & $<0.001 * * *$ \\
\hline 0 & 5484 & 35.3 & 10036 & 64.7 & \\
\hline 1 & 2513 & 33.3 & 5046 & 66.8 & \\
\hline$\geq 2$ & 1339 & 27.9 & 3465 & 72.1 & \\
\hline PCP Visits ${ }^{\mathrm{e}}$ & & & & & $<0.001 * * *$ \\
\hline Low & 5300 & 34.5 & 10054 & 65.5 & \\
\hline High & 4036 & 32.2 & 8493 & 67.8 & \\
\hline \multicolumn{6}{|c|}{ Clinical Prognostic Factors } \\
\hline Stage at Diagnosis ${ }^{c}$ & & & & & $<0.001 * * *$ \\
\hline 1 & 6250 & 39.7 & 9481 & 60.3 & \\
\hline ॥ & 2152 & 22.6 & 7362 & 77.4 & \\
\hline III & 934 & 35.4 & 1704 & 64.6 & \\
\hline Tumor Size ${ }^{c}$ & & & & & $<0.001 * * *$ \\
\hline$<1 \mathrm{~cm}$ & 5613 & 68.4 & 2588 & 31.6 & \\
\hline$<2 \mathrm{~cm}$ & 1564 & 14.6 & 9137 & 85.4 & \\
\hline $2-5 \mathrm{~cm}$ & 1932 & 24.7 & 5902 & 75.3 & \\
\hline$>5 \mathrm{~cm}$ & 227 & 19.8 & 920 & 80.2 & \\
\hline Lymph Nodes ${ }^{e}$ & & & & & $<0.001 * * *$ \\
\hline Positive & 2307 & 30.7 & 5198 & 69.3 & \\
\hline Negative & 7029 & 34.5 & 13349 & 65.5 & \\
\hline \multicolumn{6}{|c|}{ 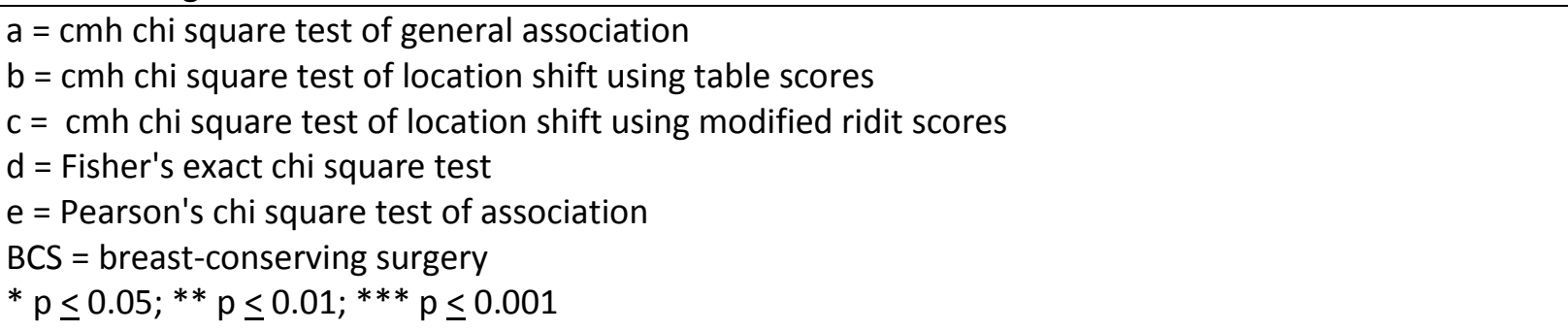 } \\
\hline
\end{tabular}


Table 2

Comparison of Guideline-Concordant Care among Elderly Women with Stages I, II, and III Breast Cancer

SEER-Medicare, 2004-2009

\begin{tabular}{|c|c|c|c|c|c|}
\hline & \multicolumn{2}{|c|}{ Concordant } & \multicolumn{2}{|c|}{ Discordant } & \multirow{3}{*}{$p$-value } \\
\hline & $\mathbf{N}$ & $\%$ & $\mathbf{N}$ & $\%$ & \\
\hline All & 9,336 & 33.5 & 18,547 & 66.5 & \\
\hline \multicolumn{5}{|l|}{ ER Status $^{\mathrm{a}}$} & $<0.001^{* * *}$ \\
\hline Positive & 7437 & 33.4 & 14861 & 66.7 & \\
\hline Negative & 1880 & 45.5 & 2256 & 54.6 & \\
\hline Borderline/Unknown & 19 & 1.3 & 1430 & 98.7 & \\
\hline \multicolumn{5}{|l|}{ PR Status ${ }^{a}$} & $<0.001 * * *$ \\
\hline Positive & 6248 & 33.3 & 12514 & 66.7 & \\
\hline Negative & 3025 & 40.5 & 4438 & 59.5 & \\
\hline Borderline/Unknown & 63 & 3.8 & 1595 & 96.2 & \\
\hline \multicolumn{5}{|l|}{ Tumor Grade } & $<0.001 * * *$ \\
\hline Well Differentiated & 2456 & 36.4 & 4285 & 63.6 & \\
\hline Moderately Differentiated & 3810 & 31.0 & 8477 & 69.0 & \\
\hline Poorly Differentiated & 2595 & 35.5 & 4707 & 64.5 & \\
\hline Undifferentiated/Unknown & 475 & 30.6 & 1078 & 69.4 & \\
\hline \multicolumn{6}{|c|}{ Tests \& Treatments } \\
\hline ER Status Tested ${ }^{d}$ & & & & & $<0.001 * * *$ \\
\hline Yes & 9336 & 35.3 & 17146 & 64.8 & \\
\hline No & 0 & 0.0 & 1401 & 100.0 & \\
\hline PR Status Tested $^{d}$ & & & & & $<0.001 * * *$ \\
\hline Yes & 9336 & 35.3 & 17099 & 64.7 & \\
\hline No & 0 & 0.0 & 1448 & 100.0 & \\
\hline Lymph Nodes Tested $^{d}$ & & & & & $<0.001 * * *$ \\
\hline Yes & 9336 & 36.4 & 16349 & 63.7 & \\
\hline No & 0 & 0.0 & 2198 & 100.0 & \\
\hline Had any Surgery ${ }^{d}$ & & & & & $<0.001 * * *$ \\
\hline Yes & 9336 & 33.6 & 18418 & 66.4 & \\
\hline No & 0 & 0.0 & 129 & 100.0 & \\
\hline Had BCS ${ }^{e}$ & & & & & $<0.001 * * *$ \\
\hline Yes & 7152 & 34.3 & 13713 & 65.7 & \\
\hline No & 2184 & 31.1 & 4834 & 68.9 & \\
\hline Had Mastectomy ${ }^{\mathrm{e}}$ & & & & & $<0.001 * * *$ \\
\hline Yes & 2651 & 22.8 & 8985 & 77.2 & \\
\hline No & 6685 & 41.2 & 9562 & 58.9 & \\
\hline
\end{tabular}

$\mathrm{a}=\mathrm{cmh}$ chi square test of general association

$\mathrm{b}=\mathrm{cmh}$ chi square test of location shift using table scores

$\mathrm{c}=\mathrm{cmh}$ chi square test of location shift using modified ridit scores

$\mathrm{d}=$ Fisher's exact chi square test

$\mathrm{e}=$ Pearson's chi square test of association

$\mathrm{BCS}=$ breast-conserving surgery

$* \mathrm{p} \leq 0.05$; $^{* *} \mathrm{p} \leq 0.01 ;{ }^{* * *} \mathrm{p} \leq 0.001$ 
Table 2

Comparison of Guideline-Concordant Care among Elderly Women with Stages I, II, and III Breast Cancer

SEER-Medicare, 2004-2009

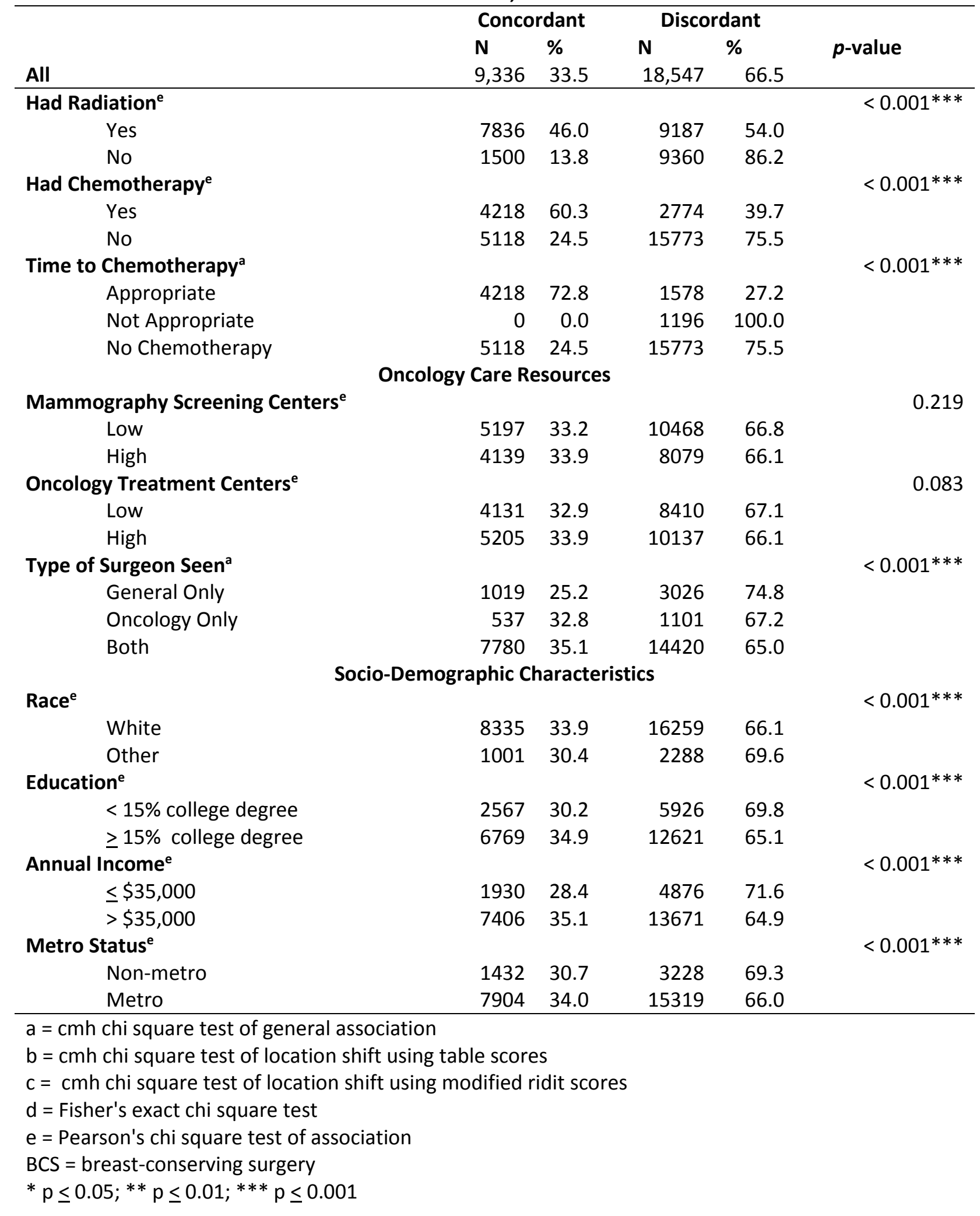


Table 3

Associations with Guideline-Concordant Care among Elderly Women with Stages I, II, and III Breast Cancer

\begin{tabular}{|c|c|c|c|}
\hline \multirow{2}{*}{ All } & & \multicolumn{2}{|c|}{ Concordant vs. Discordant } \\
\hline & AOR & $95 \% \mathrm{Cl}$ & Sig. \\
\hline \multicolumn{4}{|l|}{ Year of Diagnosis } \\
\hline 2004 & 1.00 & - & \\
\hline 2005 & 0.93 & {$[0.82,1.05]$} & \\
\hline 2006 & 0.95 & {$[0.84,1.07]$} & \\
\hline 2007 & 1.01 & {$[0.90,1.14]$} & \\
\hline 2008 & 1.09 & {$[0.98,1.21]$} & \\
\hline 2009 & 1.07 & {$[0.97,1.20]$} & \\
\hline \multicolumn{4}{|c|}{ Age \& Health } \\
\hline \multicolumn{4}{|l|}{ Age at Diagnosis } \\
\hline $66-69$ & 1.00 & - & \\
\hline $70-74$ & 0.71 & {$[0.65,0.77]$} & $* * *$ \\
\hline $75-79$ & 0.44 & {$[0.40,0.48]$} & $* * *$ \\
\hline$\geq 80$ & 0.19 & {$[0.17,0.21]$} & $* * *$ \\
\hline \multicolumn{4}{|l|}{ Comorbidity Score } \\
\hline 0 & 1.00 & - & \\
\hline 1 & 0.91 & {$[0.84,0.97]$} & $* *$ \\
\hline$\geq 2$ & 0.71 & {$[0.65,0.78]$} & $* * *$ \\
\hline \multicolumn{4}{|l|}{ PCP Visits } \\
\hline Low & 1.00 & - & \\
\hline High & 1.04 & {$[0.97,1.11]$} & \\
\hline \multicolumn{4}{|c|}{ Clinical Prognostic Factors } \\
\hline \multicolumn{4}{|l|}{ Stage at Diagnosis } \\
\hline I & 1.00 & - & \\
\hline II & 0.46 & {$[0.40,0.54]$} & $* * *$ \\
\hline III & 0.93 & {$[0.75,1.16]$} & \\
\hline \multicolumn{4}{|l|}{ Tumor Size } \\
\hline$<1 \mathrm{~cm}$ & 1.00 & - & \\
\hline$<2 \mathrm{~cm}$ & 0.05 & {$[0.04,0.05]$} & $* * *$ \\
\hline $2-5 \mathrm{~cm}$ & 0.13 & {$[0.11,0.15]$} & $* * *$ \\
\hline$>5 \mathrm{~cm}$ & 0.06 & {$[0.05,0.07]$} & $* * *$ \\
\hline \multicolumn{4}{|l|}{ Lymph Nodes } \\
\hline Positive & 1.00 & - & \\
\hline Negative & 0.49 & {$[0.44,0.56]$} & $* * *$ \\
\hline \multicolumn{4}{|l|}{ ER Status } \\
\hline Positive & 1.00 & - & \\
\hline Negative & 1.89 & {$[1.69,2.11]$} & $* * *$ \\
\hline Borderline/Unknown & 0.04 & {$[0.02,0.07]$} & $* * *$ \\
\hline
\end{tabular}

${ }^{*} p \leq 0.05 ;{ }^{* *} p \leq 0.01 ;{ }^{* * *} p \leq 0.001$ 
Table 3

Associations with Guideline-Concordant Care among Elderly Women with Stages I, II, and III Breast Cancer

\begin{tabular}{|c|c|c|c|c|}
\hline \multirow{2}{*}{ All } & & \multicolumn{3}{|c|}{ Concordant vs. Discordant } \\
\hline & & AOR & $95 \% \mathrm{Cl}$ & Sig. \\
\hline \multicolumn{5}{|c|}{ PR Status } \\
\hline & Positive & 1.00 & - & \\
\hline & Negative & 1.20 & {$[1.10,1.32]$} & $* * *$ \\
\hline & Borderline/Unknown & 0.32 & {$[0.22,0.47]$} & $* * *$ \\
\hline \multicolumn{5}{|c|}{ Tumor Grade } \\
\hline & Well Differentiated & 1.00 & - & \\
\hline & Moderately Differentiated & 1.15 & {$[1.06,1.25]$} & $* * *$ \\
\hline & Poorly Differentiated & 1.63 & {$[1.48,1.80]$} & $* * *$ \\
\hline & Undifferentiated/Unknown & 1.02 & {$[0.87,1.19]$} & \\
\hline \multicolumn{5}{|c|}{ Oncology Care Resources } \\
\hline \multicolumn{5}{|c|}{ Mammography Screening Centers } \\
\hline & Low & 1.00 & - & \\
\hline & High & 0.99 & {$[0.90,1.09]$} & \\
\hline \multicolumn{5}{|c|}{ Oncology Treatment Centers } \\
\hline & Low & 1.00 & - & \\
\hline & High & 1.01 & {$[0.91,1.12]$} & \\
\hline \multicolumn{5}{|c|}{ Type of Surgeon Seen } \\
\hline & General Only & 1.00 & - & \\
\hline & Oncology Only & 1.47 & {$[1.26,1.72]$} & $* * *$ \\
\hline & Both & 1.84 & {$[1.67,2.03]$} & $* * *$ \\
\hline \multicolumn{5}{|c|}{ Socio-Demographic Characteristics } \\
\hline \multicolumn{5}{|c|}{$\int_{0}$} \\
\hline & White & 1.00 & - & \\
\hline & Other & 0.81 & {$[0.73,0.89]$} & $* * *$ \\
\hline \multicolumn{5}{|c|}{ Education } \\
\hline & $<15 \%$ college degree & 1.00 & - & \\
\hline & $\geq 15 \%$ college degree & 1.09 & {$[1.00,1.18]$} & $*$ \\
\hline \multicolumn{5}{|c|}{ Annual Income } \\
\hline & $\leq \$ 35,000$ & 1.00 & - & \\
\hline & $>\$ 35,000$ & 1.07 & {$[0.97,1.20]$} & \\
\hline \multicolumn{5}{|c|}{ Metro Status } \\
\hline & Non-metro & 1.00 & - & \\
\hline & Metro & 1.00 & {$[0.90,1.10]$} & \\
\hline
\end{tabular}

${ }^{*} p \leq 0.05 ;{ }^{* *} p \leq 0.01 ;{ }^{* *} p \leq 0.001$ 
Table 4

Associations with Tests \& Treatment among Elderly Women with Stages I, II, and III Breast Cancer SEER-Medicare, 2004-2009

\begin{tabular}{|c|c|c|c|}
\hline \multirow[b]{2}{*}{ All } & \multicolumn{3}{|c|}{ Age $>70$ vs. Age 66-69 years } \\
\hline & AOR & $95 \% \mathrm{Cl}$ & Sig. \\
\hline \multicolumn{4}{|l|}{ Guideline-Concordant Care } \\
\hline Yes & 0.51 & {$[0.48,0.54]$} & $* * *$ \\
\hline No & 1.00 & - & \\
\hline \multicolumn{4}{|l|}{ ER Status Tested } \\
\hline Yes & 0.80 & {$[0.70,0.92]$} & $* *$ \\
\hline No & 1.00 & - & \\
\hline \multicolumn{4}{|l|}{ PR Status Tested } \\
\hline Yes & 0.78 & {$[0.68,0.89]$} & $* * *$ \\
\hline No & 1.00 & - & \\
\hline \multicolumn{4}{|l|}{ Lymph Nodes Tested } \\
\hline Yes & 0.91 & {$[0.82,1.01]$} & \\
\hline No & 1.00 & - & \\
\hline \multicolumn{4}{|l|}{ Had any Surgery } \\
\hline Yes & 2.51 & {$[1.76,3.58]$} & $* * *$ \\
\hline No & 1.00 & - & \\
\hline \multicolumn{4}{|l|}{ Had BCS } \\
\hline Yes & 0.76 & {$[0.71,0.81]$} & $* * *$ \\
\hline No & 1.00 & - & \\
\hline \multicolumn{4}{|l|}{ Had Mastectomy } \\
\hline Yes & 1.31 & {$[1.23,1.39]$} & $* * *$ \\
\hline No & 1.00 & - & \\
\hline \multicolumn{4}{|l|}{ Had Radiation } \\
\hline Yes & 0.63 & {$[0.60,0.67]$} & $* * *$ \\
\hline No & 1.00 & - & \\
\hline \multicolumn{4}{|l|}{ Had Chemotherapy } \\
\hline Yes & 0.30 & {$[0.28,0.32]$} & $* * *$ \\
\hline No & 1.00 & - & \\
\hline \multicolumn{4}{|l|}{ Time to Chemotherapy } \\
\hline Appropriate & 0.78 & {$[0.68,0.89]$} & $* * *$ \\
\hline Not Appropriate & 1.00 & - & \\
\hline No Chemotherapy & 2.71 & {$[2.38,3.09]$} & $* * *$ \\
\hline
\end{tabular}


Appendix 1. Claims Codes Used for Identifying Types of Treatment

\begin{tabular}{|c|c|c|c|c|}
\hline Type of Treatment & ICD-9 Diagnostic & ICD-9 Procedure & HCPCS/CPT & Revenue Center \\
\hline Lymph Node Surgery & & $\begin{array}{l}40.3,40.41-40.42,40.50- \\
40.54,40.59,85.43 \\
85.48\end{array}$ & $\begin{array}{l}38500,38510,38520, \\
38525,38530,39542, \\
38720,38740,38745, \\
38792,78195,78800- \\
78801\end{array}$ & \\
\hline Lumpectomy & & $85.20-85.29$ & $\begin{array}{l}19120,19125-19126, \\
19160,19162,19301- \\
19302\end{array}$ & \\
\hline Mastectomy & & $85.33-85.36,85.40-85.48$ & $\begin{array}{l}19140,19180,19182, \\
19300,19303-19307, \\
19200,19220,19240, \\
19260,19271-19272\end{array}$ & \\
\hline Radiation Therapy & V58.0, V66.1, V67.1 & $92.20-92.39$ & $\begin{array}{l}\text { 77261-77799, G0256, } \\
\text { G0261, G0173-G0174, } \\
\text { G0243, G0251, G0338- } \\
\text { G03340 }\end{array}$ & 0330,0333 \\
\hline Chemotherapy & V58.1, V66.2, V67.2, & $99.25,99.28$ & $\begin{array}{l}\text { 96400-96599, C8953- } \\
\text { C8955, G0355-G0363, } \\
\text { G902-G9032, J0640, } \\
\text { J8510, J8520-J8521, } \\
\text { J8530-J8999, J9000- } \\
\text { J9999, Q0083-Q0085, } \\
\text { S9329-S9331, }\end{array}$ & $0331,0332,0335$ \\
\hline
\end{tabular}


Appendix 1. Guideline-Concordant Treatment Options by Tumor Size and Lymph Node Status

\begin{tabular}{|c|c|c|c|c|}
\hline Stage & \multicolumn{2}{|c|}{ Treatment Option A } & \multicolumn{2}{|c|}{ Treatment Option B } \\
\hline $\begin{array}{l}\text { Stage I: } \\
\qquad \mathrm{T} \leq 1 \mathrm{~cm}, \mathrm{~N}-\end{array}$ & $\begin{array}{ll}\text { - } & \text { BCS } \\
\text { - } & \text { RT } \\
\text { - } & \text { LNT } \\
\end{array}$ & - $\quad$ ERST \& PRST & $\begin{array}{ll}\text { - } & \text { Mastectomy } \\
\text { - } & \text { LNT } \\
\text { - } & \text { ERST \& PF }\end{array}$ & \\
\hline $\begin{array}{l}\text { Stage I: } \\
\qquad 1 \mathrm{~cm}<\mathrm{T} \leq 2 \mathrm{~cm}, \mathrm{~N}-\end{array}$ & $\begin{array}{ll}\text { - } & \text { BCS } \\
\text { - } & \text { RT } \\
\text { - } & \text { LNT }\end{array}$ & $\begin{array}{ll}- & \mathrm{CT} \\
- & \mathrm{CT}<120 \text { days } \\
\text { - } & \text { ERST \& PRST }\end{array}$ & $\begin{array}{ll}\text { - } & \text { Mastectomy } \\
\text { - } & \text { LNT } \\
\text { - } & \text { CT }\end{array}$ & $\begin{array}{ll}- & \mathrm{CT}<120 \text { days } \\
- & \text { ERST \& PRST }\end{array}$ \\
\hline $\begin{array}{l}\text { Stage IIA: } \\
\qquad 0 \mathrm{~cm} \leq \mathrm{T} \leq 2 \mathrm{~cm}, \mathrm{~N}+\end{array}$ & $\begin{array}{ll}- & \text { BCS } \\
\text { - } & \text { RT } \\
\text { - } & \text { LNT } \\
\end{array}$ & $\begin{array}{ll}- & \mathrm{CT} \\
- & \mathrm{CT}<120 \text { days } \\
- & \text { ERST \& PRST } \\
\end{array}$ & $\begin{array}{ll}- & \text { Mastectomy } \\
\text { - } & \text { LNT } \\
\text { - } & \text { CT } \\
\end{array}$ & $\begin{array}{ll}\text { - } & \text { CT }<120 \text { days } \\
\text { - } & \text { ERST \& PRST }\end{array}$ \\
\hline $\begin{array}{l}\text { Stage IIA: } \\
\qquad 2 \mathrm{~cm}<\mathrm{T} \leq 5 \mathrm{~cm}, \mathrm{~N}-\end{array}$ & $\begin{array}{ll}\text { - } & \text { BCS } \\
\text { - } & \text { RT } \\
\text { - } & \text { LNT }\end{array}$ & $\begin{array}{ll}\text { - } & \mathrm{CT} \\
- & \mathrm{CT}<120 \text { days } \\
\text { - } & \text { ERST \& PRST }\end{array}$ & $\begin{array}{ll}\text { - } & \text { Mastectomy } \\
\text { - } & \text { LNT } \\
\text { - } & \text { CT }\end{array}$ & $\begin{array}{ll}\text { - } & \mathrm{CT}<120 \text { days } \\
\text { - } & \text { ERST \& PRST }\end{array}$ \\
\hline $\begin{array}{l}\text { Stage IIB: } \\
\qquad 2 \mathrm{~cm}<\mathrm{T} \leq 5 \mathrm{~cm}, \mathrm{~N}+\end{array}$ & $\begin{array}{ll}- & \text { BCS } \\
\text { - } & \text { RT } \\
\text { - } & \text { LNT } \\
\end{array}$ & $\begin{array}{ll}- & \mathrm{CT} \\
- & \mathrm{CT}<120 \text { days } \\
- & \text { ERST } \\
\end{array}$ & $\begin{array}{ll}\text { - } & \text { Mastectomy } \\
\text { - } & \text { LNT } \\
\text { - } & \text { CT } \\
\end{array}$ & $\begin{array}{ll}\text { - } & \mathrm{CT}<120 \text { days } \\
\text { - } & \text { ERST \& PRST }\end{array}$ \\
\hline $\begin{array}{l}\text { Stage IIB: } \\
\qquad \mathrm{T}>5 \mathrm{~cm}, \mathrm{~N}-\end{array}$ & $\begin{array}{ll}\text { - } & \text { Mastectomy } \\
\text { - } & \text { LNT } \\
\text { - } & \text { CT } \\
\end{array}$ & $\begin{array}{ll}\text { - } & \mathrm{CT}<120 \text { days } \\
\text { - } & \text { ERST \& PRST }\end{array}$ & & \\
\hline $\begin{array}{l}\text { Stage IIIA: } \\
\qquad 0 \mathrm{~cm} \leq \mathrm{T} \leq 5 \mathrm{~cm}, \mathrm{~N}+\end{array}$ & $\begin{array}{ll}\text { - } & \text { BCS } \\
\text { - } & \text { RT } \\
\text { - } & \text { LNT } \\
\end{array}$ & $\begin{array}{ll}\text { - } & \mathrm{CT} \\
\text { - } & \mathrm{CT}<120 \text { days } \\
\text { - } & \text { ERST } \\
\end{array}$ & $\begin{array}{ll}\text { - } & \text { Mastectomy } \\
\text { - } & \text { LNT } \\
\text { - } & \text { CT } \\
\end{array}$ & $\begin{array}{ll}\text { - } & \text { CT }<\text { 120days } \\
\text { - } & \text { ERST \& PRST } \\
\end{array}$ \\
\hline $\begin{array}{l}\text { Stage IIIA: } \\
\quad \mathrm{T}>5 \mathrm{~cm}, \mathrm{~N}+\end{array}$ & $\begin{array}{ll}\text { - } & \text { Mastectomy } \\
\text { - } & \text { LNT } \\
\text { - } & \mathrm{CT} \\
\end{array}$ & $\begin{array}{ll}- & \mathrm{CT}<120 \text { days } \\
- & \text { ERST \& PRST }\end{array}$ & & \\
\hline Stage IIIB \& IIIC & $\begin{array}{ll}\text { - } & \text { Mastectomy } \\
\text { - } & \text { LNT } \\
\text { - } & \text { CT } \\
\end{array}$ & $\begin{array}{ll} & \mathrm{CT}<120 \text { days } \\
- & \text { ERST \& PRST }\end{array}$ & & \\
\hline
\end{tabular}

$\mathrm{T}=$ tumor size; $\mathrm{N}=$ nodal status; $\mathrm{BCS}=$ breast-conserving surgery; $\mathrm{RT}=$ radiation therapy; $\mathrm{LNT}=$ lymph node testing; ERST = estrogen receptor status testing; PRST = progesterone receptor status testing; $\mathrm{CT}=$ chemotherapy; 
CHAPTER 4 


\title{
CHAPTER 4
}

"Overall 5-Year Survival among Elderly Women with Invasive Non-Metastatic Breast Cancer in a SEER-Medicare Population According to Clinical, Treatment, and Individual Characteristics"

\author{
Traci LeMasters ${ }^{1}$ \\ S. Suresh Madhavan, M.B.A, Ph.D. Chair ${ }^{1}$ \\ Usha Sambamoorthi Ph.D. ${ }^{1}$ \\ Dustin Long Ph.D. ${ }^{2}$ \\ Hannah Hazard M.D. ${ }^{3}$ \\ Kimberly M Kelly Ph.D. ${ }^{1,4}$
}

1 Department of Pharmaceutical Systems and Policy, School of Pharmacy, West Virginia University

${ }^{2}$ Department of Biostatistics, School of Public Health, West Virginia University

${ }^{3}$ Mary Babb Randolph Cancer Center, West Virginia University

${ }^{4}$ Department of Medical Education, School of Medicine, West Virginia University 


\section{Abstract}

Objectives: Overall breast cancer survival has improvement dramatically over the past 20 years and survival rates are relatively high, especially when detected at an early stage. Still, several factors remain formidable influences on breast cancer survival. This study examined factors associated with overall 5-year breast cancer survival among three different large groups of elderly US women with non-metastatic invasive breast cancer.

Methods: The linked SEER-Medicare data set was used to identify 1) a broadly inclusive group of women age $\geq 66$ years diagnosed with stage I, II, and II breast cancer in 2003 to 2009 ( $\mathrm{N}=$ $53,830), 2$ ) women age $\geq 66$ years diagnosed with early-stage invasive breast cancer in 2003 to $2009(\mathrm{~N}=45,981)$ by initial loco-regional treatment patterns (breast-conserving surgery (BCS) plus radiation therapy (RT), mastectomy or BCS without RT), and 3) women age $\geq 66$ years with pathologically staged I, II, and III breast cancer in 2004 to $2009(\mathrm{~N}=27,883)$ by individual tests and treatments receipt of guideline-concordant care (GCC), among fee-for-service beneficiaries continuously enrolled in Part A and B services. Five-year survival rates, adjusted hazard ratios, and Kaplan Meier survival curves were computed for each sample according to study covariates. Results: Among elderly women in cohort 1 , the average time till death was $1,247.2$ days $(S D= \pm$ 722.3) with an $82 \%$ 5-year survival rate. Groups with the highest 5-year survival rates were age 66-69 years $(91 \%)(P<0.001)$, with a comorbidity score $=0(86 \%)(P<0.001)$, and diagnosed at stage I $(88 \%)(P<0.001)$. Groups with the greatest mortality risk within 5 years of diagnosis were those age $\geq 80$ years $(\mathrm{AHR}, 4.01 ; 95 \% \mathrm{CI}, 3.74,4.29$ ), with a comorbidity score $\geq 2$ (AHR, 2.17; 95\% CI, 2.06, 2.28), and diagnosed at stage III (AHR, 3.95; 95\% CI, 3.74, 4.18). In cohort 2, the average time till death was $1,410.6$ days $(S D= \pm 696.9)$ with $86 \%$ 5-year survival rate. Groups with highest 5-year survival rates were those who were age 66-69 years $(94 \%)(P<$ 
0.001), with a comorbidity score $=0(90 \%)(P<0.001)$, diagnosed at stage I $(90 \%)(P<0.001)$, treated by both an oncology surgeon $(87 \%)(P<0.001)$ or both an oncology and general surgeon $(88 \%)(P<0.001)$, and received BCS plus RT $(92 \%)(P<0.001)$. Groups of women with the greatest mortality risk within 5 years of diagnosis were those age $\geq 80$ years (AHR, 3.76; $95 \%$ CI, 3.45, 4.10), a comorbidity score $\geq 2$ (AHR, 2.39; 95\% CI, 2.25, 2.55), and had BCS without RT (AHR, 1.94; 95\% CI, 1.82, 2.06). In cohort 3 the average time till death was 1,246.7 days $(S D= \pm 600.8)$ with a $88 \%$ 5-year survival rate. Groups with the highest 5-year survival rates were those age 66-69 years $(94 \%)(P<0.001)$, with a comorbidity score $=0(91 \%)(P<0.001)$, a low frequency of primary care visits $(90 \%)(P<0.001)$, diagnosed at stage I $(93 \%)(P<$ 0.001), lymph node negative $(91 \%)(P<0.001)$, ER positive $(89 \%)(P<0.001)$, PR positive $(90 \%)(P<0.001)$, treated by an oncology surgeon $(90 \%)(P<0.001)$ or both an oncology and general surgeon $(88 \%)(P<0.001)$, received GCC $(93 \%)(P<0.001)$, and had RT $(91 \%)(P<$ 0.001). Groups of women with the greatest risk within 5 years of diagnosis were those age $\geq 80$ years (AHR, 2.85; 95\% CI, 2.54, 3.20), a comorbidity score $\geq 2(\mathrm{AHR}, 2.09 ; 95 \% \mathrm{CI}, 1.92$, 2.27), diagnosed at stage III (AHR, 1.81; 95\% CI, 1.48, 2.21), received GCC (AHR, 1.18; 95\% CI, 1.04, 1.35), and did not have RT (AHR, 1.53; 95\% CI, 1.39, 1.68).

Conclusions: Across all three study samples, age, health, stage at diagnosis, and type of treatment, were strong and consistent factors associated with overall 5-year breast cancer survival. Undertreatment among elderly women with breast cancer was associated with a greater risk of death within 5 years of diagnosis, particularly for women who did not receive RT following BCS for early-stage breast cancer or receive GCC for all invasive non-metastatic. In order to increase survival, older women with breast cancer should receive aggressive treatment in accordance with recommended guidelines as long as they are healthy enough to do so. 
Modifiable factors such as stage at diagnosis should continue to be targeted by encouraging persistent mammography screening among elderly women. Reducing comorbidity burden among US women would be an effective means for improving overall breast cancer survival among all groups of women, and especially among older women whose vitality and functional status is already at risk for decline. 


\section{Introduction}

While breast cancer is the most frequently diagnosed type of cancer among women in the United States (US), it also has among the highest survival rates, relative to other types of cancer. Relative to women who have never been diagnosed with breast cancer, breast cancer survivors have $89 \%$ 5-year and $83 \%$ 10-year survival rates. ${ }^{1,2}$ Moreover, earlier stage diagnosis is associated with greater survival, such that women diagnosed at Stage I, II, and III have 100\%, $93 \%$, and $72 \%$ 5-year survival rates, respectively. ${ }^{3}$ Decreasing mortality and increasing survival rates throughout the years are attributed to improvements in understanding of the disease and its treatment, alongside earlier detection due to increased routine breast cancer screening. ${ }^{4}$ Yet, despite these advancements, several factors still affect survival outcomes. These factors encompass clinical characteristics of the cancer, receiving the standard of oncology care, such that treatment is in concordance with evidence based recommended treatment guidelines without the omission of necessary tests and treatments, otherwise referred to as guideline-concordant care (GCC), age, health, access to specialty care, and socio-demographic characteristics. Clinical characteristics associated with greater survival are earlier stage at diagnosis, smaller tumor size, less or no lymph node invasion, well differentiated tumor grade, and estrogen and progesterone positive tumors. ${ }^{3,5-7}$ Receiving GCC is associated with greater breast cancer survival, while guideline-discordant care (GDC), particularly the omission of recommended treatments and testing, is associated with poorer survival. ${ }^{8-11}$ The most commonly omitted treatments are radiation therapy (RT) following breast-conserving surgery (BCS) or mastectomy when indicated, chemotherapy, adjuvant hormone therapy, and lymph node sampling. The appropriate course of treatment for each women is determined by tumor size, lymph node invasion, and hormone receptor status. When diagnosed at an early-stage, women often have an option for the 
type of local treatment received. Women with tumors $\leq 5 \mathrm{~cm}$ have the option of mastectomy or BCS followed by RT. Multiple randomized clinical studies have shown BCS followed by RT to be as equally effective as mastectomy for long-term survival of invasive early-stage breast cancer. ${ }^{12-15}$ Since the National Institutes of Health's (NIH) 1991 endorsement of BCS plus RT as an appropriate alternative to mastectomy for treatment of invasive early-stage breast cancer, mastectomy rates have decreased while rates of BCS plus RT have steadily increased. ${ }^{16}$ In fact, BCS, with or without RT, has become the preferred choice of primary surgical treatment among women with early-stage breast cancer, with $\sim 60 \%$ or more receiving BCS. ${ }^{17-19}$ Yet, in recent years, rates of mastectomy have started to slowly increase. ${ }^{18-20}$ Associations with receipt of mastectomy when BCS is an option, include a desire to avoid radiation therapy due to side effects, fear of recurrence, larger tumor size, positive lymph nodes, tumors with negative hormone receptors, moderately or poorly differentiated tumors, lower income, being insured by Medicaid or uninsured, residing in a rural location, increased travel distance for RT, the increased cost of BCS plus RT, compared to mastectomy, and surgeon characteristics that include being male, not trained in the U.S., and less recent training. ${ }^{18,19,21-25}$

Regardless of stage or treatment options, there are several factors that are indirectly associated with survival via their association with receipt of GCC. Age and comorbidity are two related and strongly associated predictors of GCC. While increasing age has its own associated frailty, it is also associated with increasing comorbidity, that together, may make some treatments with adverse and toxic effects intolerable for some women, to the point that the harms of such treatments outweigh the benefits. ${ }^{26-31}$ In fact, clinical evidence-based treatment guidelines put forth by the National Comprehensive Cancer Network (NCCN) do not make recommendations for or against chemotherapy in women age $>70$ years, but suggest 
chemotherapy be given on a case-by-case basis, depending on the patient's health. ${ }^{32}$ Additionally, guidelines specify that RT following BCS may be omitted among women meeting the following requirements: age $\geq 70$ years, tumors $\leq 2 \mathrm{~cm}$, node negative, estrogen-receptor positive, and receiving adjuvant hormone therapy. ${ }^{33}$ This change to recommended treatment guidelines that had previously recommended RT following BCS for all age groups, was made after the 2004 publication of the Cancer and Leukemia Group B (CALGB) C9343 trial study results demonstrated similar 5-year survival between women with these clinical characteristics who received BCS plus RT or BCS alone. However, since this change in treatment recommendations, evidence suggests that a greater number of elderly women do not receive RT or chemotherapy, than those that meet exemption requirements from RT or who are in too poor of health to undergo chemotherapy. ${ }^{34-36}$ Women's receipt of GCC is often affected by their access to oncology services due to their location of residence and socio-demographic characteristics. Women residing in rural vs. urban areas, in areas with a lower density of oncology service providers, and that have greater distances to travel for care are more likely to not receive adjuvant treatments such as RT and chemotherapy, or delay the initiation of treatment. ${ }^{26,36-38}$ Women who are of black or non-white Hispanic race, lower income, lower education, publically insured or uninsured are less likely to receive GCC. ${ }^{27,39,40}$ Race is also directly associated with survival outcomes. Black women have markedly worse survival outcomes, as compared to white women, such that 5-year survival for white and black women is $90 \%$ and $79 \%$, respectively. 1,41,42 In addition to vulnerable socio-demographic factors that decrease the likelihood of receiving GCC, black women diagnosed with breast cancer are more to have tumors with less favorable characteristics and aggressive cancers. ${ }^{43,44}$ 


\section{$\underline{\text { Statement of the Problem }}$}

While one can see from this review of the literature that numerous studies on breast cancer survival have been conducted throughout the years, the data sources, subsets of populations, stage at diagnosis, year of diagnosis, treatment patterns, and covariates, have also been numerous and of wide ranging. In contrast, this study seeks to provide a comprehensive understanding of overall breast cancer survival by examining all of the previously discussed factors associated with survival using large, nationally representative data set with recent survival data. Moreover, this study will focus on the survival of elderly women with breast cancer, who are uniquely vulnerable to worse survival outcomes due to increasing age and comorbidity.

Keeping these thoughts in mind, this study aims to examine overall 5-year survival and age, health, clinical prognostic factors, access to oncology care resources, socio-demographic characteristics, and treatment patterns among three different cohorts of elderly women with breast cancer sampled from the SEER-Medicare linked dataset. Survival analysis of the first cohort, a broadly inclusive group of women with stage I, II, and III breast cancer, will examine how age, health, clinical prognostic factors, and socio-demographic characteristics are associated with overall 5-year survival and provide a baseline understanding of survival as a cohort and by stage. The second cohort analyses will examine survival among women with early-stage (stage I and II) breast cancer according to the factors listed in cohort 1, and by type of loco-regional treatment (BCS plus RT, mastectomy, and BCS without RT). Building upon the first two analysis, the third cohort analysis will additionally determine how receipt of individual tests, treatments, and GCC vs. GDC are differentially associated with overall 5-year survival among elderly women with pathologically staged I, II, and III breast cancer. 


\section{Methods}

\section{Data \& Study Design}

This retrospective cohort study was conducted using the linked SEER-Medicare dataset. The SEER-Medicare database was created in a collaborative effort by the National Cancer Institute (NCI) and Centers for Medicare and Medicaid Services (CMS) to combine clinical information gathered by the Surveillance Epidemiology and End Results cancer registry program with Medicare insurance claims. The SEER program collects basic socio-demographic, clinical, and vital status information on incident cancer cases from multiple reporting sources. This information is found the Patient Entitlement and Diagnosis Summary File (PEDSF). SEER data used for this study consisted of 17 population-based registries, representing $26 \%$ of the U.S. population. An algorithm based on name, Social Security number, sex, and date of birth successfully matched $94 \%$ SEER cases to their Medicare claims beginning from the time of eligibility till death. ${ }^{45}$ This study used Medicare claims providing information regarding beneficiary enrollment (Denominator File), hospital inpatient claims (MedPAR file), hospital outpatient claim (Outpatient file), physician office visits ( $\mathrm{NCH}$ file), and durable medical equipment (DME file). ${ }^{46}$ Additionally, county and state of diagnosis were used to identify the area-level density of mammography screening and oncology centers from the U.S. Department of Health and Human Resource's 2009 Area Resource File (ARF). ${ }^{47}$

\section{$\underline{\text { Study Populations }}$}

\section{Cohort 1: All Women with Stage I, II, \& III Breast Cancer}

This study sample consisted of American Joint Commission on Cancer (AJCC) clinically and/or pathologically staged I, II, and III incident cases of breast cancer recorded in SEER during years 2003 to 2009, among female age-eligible Medicare beneficiaries who were 
continuously enrolled in Medicare Part A and B fee-for-service programs 1 year before and after diagnosis, and were therefore age $\geq 66$ years at the time of diagnosis. Women were excluded if they were enrolled in a managed care program at any time during year before to the year after diagnosis, diagnosed at the time of death or upon autopsy, had end-stage renal disease, did not have primary surgical treatment, or surgeon specialty was missing. The final sample size was $\mathrm{n}=$ 53,830 elderly women.

Cohort 2: Local Treatment among Women with Stage I \& II Breast Cancer

This study sample consisted of American Joint Commission on Cancer (AJCC) clinically and/or pathologically staged I and II incident cases of breast cancer recorded in SEER during years 2003 to 2009, among female age-eligible Medicare beneficiaries who were continuously enrolled in Medicare Part A and B fee-for-service programs 1 year before and after diagnosis, and were therefore age $\geq 66$ years at the time of diagnosis. Women were excluded if they were enrolled in a managed care program at any time during year before to the year after diagnosis, diagnosed at the time of death or upon autopsy, had end-stage renal disease, did not have primary surgical treatment, or surgeon specialty was missing. The final sample size was $n=45,981$ elderly women.

Cohort 3: Guideline-Concordant Care among Women with Stage I, II, \& III Breast Cancer This study sample consisted of American Joint Commission on Cancer (AJCC) pathologically staged I, II, and III incident cases of breast cancer recorded in SEER during years 2004 to 2009, among female age-eligible Medicare beneficiaries who were continuously enrolled in Medicare Part A and B fee-for-service programs 1 year before and after diagnosis, and were therefore age $\geq 66$ years at the time of diagnosis. Women were excluded if they were enrolled in a managed care program at any time during year before to the year after diagnosis, diagnosed at 
the time of death or upon autopsy, died within the first year of diagnosis, had end-stage renal disease, did not have primary surgical treatment, or surgeon specialty was missing. The final sample size was $n=27,883$ elderly women.

\section{$\underline{\text { Measures }}$}

Dependent Variable

Overall survival is measured by examining all-cause mortality (breast cancer specific and all other causes of death). Survival outcomes measured were average time-to-death in days and 5-year survival as calculated by subtracting the date of diagnosis from the Medicare date of death (DOD) or end of follow-up. Medicare DOD is captured from CMS's Master Enrollment (EDB) and MedPAR data files. The EDB obtains information regarding DOD from the Social Security Administration (SSA). The DOD is flagged as a "valid" date if it has been validated by a death certificate. If the DOD has not been validated then the DOD is set the last day of the month in which the beneficiary died. MedPAR records the DOD for those who died in a hospital. SEER registries also collect information regarding DOD from state death certificates and other sources. SEER and Medicare DOD information are in $99.5 \%$ level of agreement with one another. Observations were censored for women who were alive as of February 29, 2012. Independent Variables

Specific to cohort 2, initial loco-regional treatment received for early-stage breast cancer (BCS plus RT, mastectomy, or BCS only) was identified using surgical and radiation claims where the claim date was no later than 366 days from the date of diagnosis. Surgery claims were identified using the Medicare Provider and Analysis Review (MEDPAR), Physician/Supplier (NCH), and Outpatient files using International Classification of Diseases, 9th Revision (ICD-9) procedure codes, Healthcare Common Procedure Coding System (HCPCS), and Current 
Procedural Terminology (CPT) to identify BCS (i.e. lumpectomy, partial mastectomy, segmental mastectomy) and mastectomy. When multiple claims for lumpectomy were present, or when a claim for lumpectomy was followed by a claim for mastectomy, only the first surgical claim was used, to identify initial choice for primary surgical treatment. Receipt of RT was identified from MEDPAR, NCH, Outpatient, and DME files using ICD-9 diagnosis codes, ICD-9 procedure codes, and HCPCS/CPT codes. Only the first claim was used to identify the initiation of RT.

Specific to cohort 3, GCC vs. guideline-discordant care (GDC) was determined by comparing the correct course of care according to tumor size and nodal status of each women as per NCCN evidence-based clinical practice guidelines, to the actual care received (Table 1). Care was also determined to be guideline-concordant if chemotherapy was initiated within 120 days of diagnosis, when chemotherapy was indicated, as per ASCO/NCCN quality measures, and if lymph node invasion, and hormone receptor statuses were tested. Although ASCO/NCCN quality measures indicate RT should be initiated within 1 year of diagnosis, this was not considered in the determination of GCC, as all claims used for this study were made within 1 year after diagnosis. For this study purpose, being of age $\geq 70$ years was not considered a determining factor for GCC vs. GDC when RT or chemotherapy was indicated, in order to determine the extent that younger and older women receive differential care.

Within cohort 3, receipt of individual treatments and tests were also examined and included lymph node testing (yes or no), ER status testing (yes or no), PR status testing (yes or no), had any primary surgery (BCS or mastectomy) (yes or no), had BCS (yes or no), had mastectomy (yes or no), had RT (yes or no), had chemotherapy (yes or no), and chemotherapy was initiated within 120 day of diagnosis (yes, no, or not applicable). Hormone receptor status testing was assessed using the status given by the ER status and PR status variable in the Patient 
Entitlement and Diagnosis Summary File (PEDSF) file. If the status was listed as "positive", "negative", or "borderline", then the receptor status was determined to have been tested. If the status was listed as "unknown", then it was considered as not having been tested. Lymph node testing was defined by the claims codes for axillary lymph node dissection (ALND) or sentinel lymph node (SNB) biopsy. Surgical, RT, and chemotherapy treatments were determined using ICD-9 diagnostic and procedure, HCPCS/CPT codes found in the MEDPAR, Outpatient, and NCH data files. All claims codes are shown in Appendix 1.

Others measures examined in relation to survival were year of diagnosis, age at diagnosis $(66-69,70-74,75-79, \geq 80)$, health, clinical prognostic factors, oncology care resources, and socio-demographic characteristics. Age was also dichotomized (66-69 or $\geq 70)$ to estimate the probability of receipt of individual tests and treatments between younger and older elderly women in the third cohort. Health was measured examining patient comorbidity and frequency of primary care provider (PCP) visits. Patient comorbidity was calculated by applying an algorithm to Medicare claims that identifies a comorbid condition as the presence of at least one in-patient or two out-patient claims using ICD-9 diagnoses codes for each co-morbid condition. Overall comorbidity was then classified using the Klabunde adaption of the Charlson comorbidity index score $(0,1, \geq 2) \cdot{ }^{48,49}$ An index of PCP visit intensity was identified by counting of number of unique PCP claim dates the year before diagnosis in the $\mathrm{NCH}$ file. $\mathrm{PCP}$ visits were categorized by lower and upper 50th percent median cutoff (low, high). Clinical prognostic factors examined were stage diagnosis (I, II, III) using the AJCC Stage Group 6th edition. Women in the cohort 3, were limited to those who were pathologically staged. Other clinical measures included tumor size $(<1 \mathrm{~cm},<2 \mathrm{~cm}, 2-5 \mathrm{~cm},>5 \mathrm{~cm})$, lymph node status (positive or negative), estrogen-receptor (ER) status, progesterone-receptor (PR) status (positive, 
negative, borderline/unknown) and tumor grade (well differentiated, moderately differentiated, poorly differentiated, undifferentiated/unknown). Access to oncology care resources was examined using the density of area-level mammography screening and oncology treatment centers, and surgeon specialty. The density of area-level mammography screening centers and oncology treatment centers, relative to each women, was measured using data from the ARF and categorized lower and upper 50th percent median cutoff (low, high). Surgeon specialty was assessed using provider specialty claims codes 02,49 (general) and 83, 90, 91, 98 (oncology) from the $\mathrm{NCH}$ file to determine the type of surgeon seen (general only, oncology only, both). Socio-demographic characteristics examined were race (white or other), education (measured by the 2000 Census tract survey of percent of persons age $\geq 25$ with at least 4 years college education) $(<15 \%, \geq 15 \%)$, annual income (measured by the 2000 Census tract survey of median income by census) $(\leq \$ 35,000,>\$ 35,000)$, and metro status (non-metro, metro). Analysis

Descriptive analysis was performed for all study variables. Mantel-Haenszel chi-square tests of location shift using modified ridit scores or table scores and Mantel-Haenszel chi-square tests of general association (depending on whether the independent measure was dichotomous, nominal, or ordinal) were used to test for significant group differences between independent measures and women who were dead or alive 5 years after diagnosis, with significance set at $P \leq$ .05. Cox proportional hazards regression models were used to estimate the hazard, or risk, of death at any time within 5 years of diagnosis, adjusting for independent variables. Parameter estimates calculated in the regression models are presented as adjusted hazard ratios (AHR) with their corresponding 95\% confidence intervals (CI). The probability of death from time of diagnosis till end of follow-up was also estimated via survival functions for each study cohort 
and stratified by stage at diagnosis, type of loco-regional treatment, and receipt of GCC using the Kaplan Meier method. All analysis were conducted using SAS version 9.4 software (SAS Institute Inc., Cary, NC).

\section{Results}

Cohort 1: All Women with Stage I, II, \& III Breast Cancer

Cohort Characteristics

Among elderly women diagnosed with stage I, II, and III breast cancer in 2003 to 2009, the average time-to-death was 1,247.2 $(S D= \pm 722.3)$ days. The majority were $\geq 80$ years old (30.0\%), had a comorbidity score $=0(56.3 \%)$, had a low frequency of PCP visits $(54.6 \%)$, diagnosed at stage I (56.0\%), had ER positive (77.7\%) and PR positive (64.8\%) tumors, moderately differentiated tumor grades $(42.7 \%)$, lived in areas with a low density of mammography screening centers $(51.6 \%)$ and oncology treatment centers $(56.0 \%)$, were treated by both a general and oncology surgeon $(75.7 \%)$, white $(88.6 \%)$, lived in metro areas $(83.4 \%)$, areas where $15 \%$ or more of the population was college educated $(69.9 \%)$ or where the average annual income was greater than $\$ 35,000(75.7 \%)$.

Group Differences in 5-Year Survival

All group comparisons (shown in Table 3) were significant at the $P \leq 0.001$ level. The average time-to-death between women who were alive and dead 5 years after diagnosis was 2,327.2 days $(S D= \pm 357.6)$ vs. 938.9 days $(S D= \pm 454.1)(P<0.001)$. Age \& Health

As age at diagnosis increased, 5-year survival rates decreased, with $91.3 \%$ of women diagnosed at $66-69$ years vs. $66.5 \%$ of women diagnosed at age $\geq 80$ years still living. Five-year survival rates decreased with increasing comorbidity, such that $86.2 \%$ of women with a 
comorbidity score $=0$ vs. $69.6 \%$ of women with a comorbidity score $\geq 2$ were still alive 5 years after diagnosis. Women with a lower frequency of PCP visits $(84.5 \%)$ had higher 5-year survival rates than women with a higher frequency of visits $(78.1 \%)$.

\section{Clinical Prognostic Factors}

Women diagnosed at stage I $(88.3 \%)$ had higher 5-year survival rates than those diagnosed at stage II (77.4\%) and III (58.1\%). Women whose tumors were ER and PR positive $(84.1 \%$ \& $84.9 \%)$ had higher 5-year survival rates, than those that were ER and PR negative $(72.0 \% \& 75.8 \%)$. Women with well differentiated tumors also had higher 5-year survival rates $(88.1 \%)$, than those who were moderately $(83.8 \%)$, poorly $(73.3 \%)$ differentiated, or undifferentiated/unknown (75.9\%).

\section{Oncology Care Resources}

Women who resided in areas with higher densities of mammography screening centers $(82.1 \%)$ and oncology treatment centers $(82.4 \%)$ had slightly higher 5-year survival rates, than those living in lower density areas $(81.0 \%$ \& 80.9\%, respectively). Higher 5-year survival rates were observed among women treated by and oncology surgeon $(82.4 \%)$ or both a general and oncology surgeon (83.6\%), as compared to women treated by a general surgeon only $(73.5 \%)$. Socio-Demographic Characteristics

In regards to race, $81.8 \%$ of white women vs. $79.4 \%$ of women of other race were still alive 5 years after diagnosis. Women residing in areas where the population was $\geq 15 \%$ college educated $(82.8 \%)$ and had average income $>\$ 35,000(82.9 \%)$ had higher 5-year survival rates than those living in areas where $<15 \%$ was college educated $(78.6 \%)$ and the average income was $\leq \$ 35,000(77.2 \%)$. Similarly, women residing in metro areas $(82.0 \%)$ had higher survival rates, than those living in non-metro areas $(79.6 \%)$. 
Risk of Death within 5 Years of Diagnosis

Age \& Health

Adjusted hazard ratios estimating the risk of death 5 years after diagnosis according to study covariates are presented in Table 4 . The risk of death at any time within 5 years of diagnosis increased substantially as age at diagnosis increased, with women diagnosed at 70-74 years, $75-79$ years, and $\geq 80$ years being $24 \%$ (AHR, 1.24; 95\% CI, 1.15, 1.35), 86\% (AHR, 1.86; 95\% CI, 1.73, 2.01), and over 400\% (AHR, 4.01; 95\% CI, 3.74, 4.29) more likely to die than women diagnosed at age 66-69 years. Compared to women with a comorbidity score $=0$, those with a score $=1$ or score $\geq 2$ were $1.44(95 \% \mathrm{CI}, 1.37,1.51)$ and $2.17(95 \% \mathrm{CI}, 2.06,2.28)$ times more likely to die within 5 years of diagnosis. Similarly, women with a high frequency of PCP visits were 1.14 times more likely to die (95\% CI, 1.10, 1.19), than women with a low frequency of visits, adjusting for all other variables.

\section{Clinical Prognostic Factors}

Later stage at diagnosis was strongly associated with 5-year survival, such that women diagnosed at stage II and stage III had an $82 \%$ (AOR, 1.82; 95\% CI, 1.74, 1.91) and 395\% $(\mathrm{AOR}, 3.95 ; 95 \% \mathrm{CI}, 3.74,4.18)$ greater risk of death at any time within 5 years of diagnosis, compared to women diagnosed at stage I. Women with tumors that were ER and PR negative were $1.36(95 \% \mathrm{CI}, 1.27,1.45)$ and $1.19(95 \% \mathrm{CI}, 1.12,1.26)$ times more likely to die within 5 years of diagnosis, than women with ER and PR positive tumors. Compared to women with well differentiated tumors, those with moderately, poorly, and undifferentiated/unknown tumors were $1.14(95 \% \mathrm{CI}, 1.07,1.21), 1.54(95 \% \mathrm{CI}, 1.44,1.65)$, and 1.38 (95\% CI, 1.27, 1.50) times more likely to die within 5 years of diagnosis. 


\section{Oncology Care Resources}

Compared to women treated by a general surgeon, those who were treated by an oncology surgeon or both an oncology and general surgeon had a 22\% (AOR, 0.78; 95\%, 0.72, $0.86)$ and $32 \%(\mathrm{AOR}, 0.68 ; 95 \%, 0.65,0.71)$ decreased risk of death within 5 years of diagnosis, adjusting for all other independent variables.

\section{Socio-Demographic Characteristics}

Women residing in areas where $\geq 15 \%$ of the population was college educated were 0.92 times less likely $(95 \% \mathrm{CI}, 0.88,0.99)$ to die within 5 years of diagnosis, than women living in less college educated areas. Similarly, women residing in areas with an average income > $\$ 35,000$ were also less likely to die within 5 years of diagnosis.

Kaplan Meier Estimates

Five-year overall survival rates for all women in cohort 1and stratified by stage at diagnosis are shown in Figures 1 and 2, with the number at risk for death given in 366 day intervals. Figure 2 shows that women with earlier stage at diagnosis have significantly greater probability of overall 5-year survival at all time points, than those with later stage at diagnosis $(P$ $<0.001)$.

\section{Cohort 2: Local Treatment among Women with Stage I \& II Breast Cancer}

\section{Cohort Characteristics}

The average time to death was $1,410.6$ days $(S D= \pm 696.9)$ among women diagnosed with early-stage breast cancer in 2003 to 2009 . The majority of elderly women were age $\geq 80$ years at diagnosis $(28.7 \%)$, had a comorbidity score $=0(56.7 \%)$, and a low frequency of PCP visits (54.0\%). About $63 \%$ of women were diagnosed at stage I, most had ER (79.1\%) and PR $(66.3 \%)$ positive tumors, and moderately differentiated (43.6\%) tumors. A little over half of 
women lived in areas with a low density of mammography screening centers $(51.7 \%)$, and low density of oncology treatment centers $(56.0 \%)$. The majority of women were treated by both a general and oncology surgeon $(75.6 \%)$. In regards to socio-demographics, most women were white $(89.4 \%)$, lived in metro areas $(83.3 \%)$, areas where the population was $\geq 15 \%$ college educated (70.5\%), and had an annual income $>\$ 35,000(76.4 \%)$. More than half of the women received BCS plus RT (54.5\%) as their initial loco-regional treatment, while $23.4 \%$ had mastectomy and $22.1 \%$ had lumpectomy without RT.

Group Differences in 5-Year Survival

All group comparisons between women who were alive or dead 5 years after diagnosis were significant at the $P \leq 0.001$, except race $(P=0.092)$. Women still living 5 years after diagnosis had a significantly greater time-to-death $(M=2,338.7$ days, $S D= \pm 361.6)$, than those who died $(M=1055.1$ days, $S D= \pm 406.5)(P<0.001)$.

Age \& Health

Survival rates decreased with increasing age. About $94 \%$ of women age 66-69 years vs. $73.3 \%$ of women age $\geq 80$ years were still living 5 years after diagnosis. Those with a comorbidity score $=0$ had higher 5 -year survival rates $(89.9 \%)$, than women with a score $=1$ (79.8\%) and score $\geq 2(75.2 \%)$. Compared to women with a high frequency of PCP visits $(82.8 \%)$, women with a low frequency of PCP visits had higher 5-year survival rates $(88.6 \%)$.

\section{Clinical Prognostic Factors}

Elderly women diagnosed at stage I had an $89.5 \%$ 5-year survival rate, whereas women diagnosed at stage II had a 79.9\% 5-year survival rate. Similar differences were observed between positive and negative tumor receptors. Those with ER and PR positive tumors had $87.6 \%$ and $88.1 \%$ 5-year survival rates, in contrast to $79.1 \%$ and $81.9 \%$ survival rates among 
those with ER and PR negative tumors. Women with well-differentiated tumor grades had the highest 5-year survival rates $(89.9 \%)$, compared to women with moderately $(86.9 \%)$, poorly $(80.2 \%)$, and undifferentiated/unknown tumor grades $(85.0 \%)$.

\section{Oncology Care Resources}

Women residing in areas with a high density of mammography screening centers and oncology treatment centers had slightly higher 5-year survival rates $(86.5 \% \& 86.7 \%)$, than those residing in lower density areas $(85.4 \% \& 85.4 \%$, respectively). Whereas women who were treated by an oncology surgeon (87.3\%) or both an oncology and general surgeon had higher 5year survival rates $(87.8 \%)$, than those treated by a general surgeon $(78.0 \%)$.

\section{Socio-Demographic Characteristics}

Women who resided in metro vs. non-metro areas (86.3\% vs. $84.3 \%)$, areas where $\geq 15 \%$ of the population was college educated vs. $<15 \%$ college educated $(87.0 \%$ vs. $83.5 \%)$ or annual income $>\$ 35,000$ vs. $\leq \$ 35,000(87.0 \%$ vs. $82.6 \%)$ had higher 5-year survival rates.

\section{Treatment}

Women who were received BCS plus RT as their initial loco-regional treatment had higher 5-year survival rates at almost 92\%, compared to women who were treated with mastectomy $(79.3 \%)$ or BCS only $(78.2 \%)$.

\section{$\underline{\text { Risk of Death within } 5 \text { Years of Diagnosis }}$}

\section{Age \& Health}

Women who age 70-74, 75-79, and $\geq 80$ years had a $21 \%$ (AHR, 1.21; 95\% CI 1.10, 1.34), 90\% (AHR, 1.90; 95\% CI, 1.73, 2.09), and 376\% (AHR, 3.76; 95\% CI, 3.45, 4.10) increased risk of death within 5 years of diagnosis, adjusting for all other independent variables, than women age 66-69 years at diagnosis. Compared to women with a comorbidity score $=0$, 
those with a score $=1$ or score $\geq 2$ were $1.49(95 \%$ CI, 1.41, 1.58) and $2.39(95 \%$ CI, 2.25, 2.55) times more likely to die within 5 years of diagnosis.

\section{Clinical Prognostic Factors}

Women diagnosed at stage II had a $65 \%$ increased risk of death within 5 years of diagnosis (AHR, 1.66; 95\% CI, 1.57, 1.74), compared to women diagnosed at stage I. Women with ER and PR negative tumors were 33\% (AHR, 1.33; 95\% CI, 1.23, 1.45) and 17\% (AHR, 1.17; $95 \%$ CI, 1.09, 1.26) more likely to die within 5 years of diagnosis. Having poorly differentiated tumors increased the hazard of death by $49 \%$ (AHR, 1.49; 95\% CI, 1.38, 1.61), while having moderately tumors increased the hazard of death by $15 \%$ (AHR, $1.15 ; 95 \% \mathrm{CI}$, $1.08,1.24)$, compared well differentiated tumors.

\section{Oncology Care Resources}

Women treated by an oncology surgeon or both an oncology and general surgeon had a lower risk of death within 5 years of diagnosis, (AHR, 0.81, 95\% CI, 0.72, 0.91) and (AHR, $0.72 ; 95 \% \mathrm{CI}, 0.68,0.76)$, than women treated by a general surgeon only.

\section{Socio-Demographic Characteristics}

Those who resided in areas where $\geq 15 \%$ of the population was college educated were 0.91 times less likely $(95 \% \mathrm{CI}, 0.86,0.97)$ to die within 5 years of diagnosis, than those residing in areas where $<15 \%$ of the population is college educated.

\section{Treatment}

Compared to women received BCS plus RT as their initial loco-regional treatment for early-stage breast cancer, those who had BCS without RT had a 94\% (AOR, 1.94; 95\% CI, 1.82, $2.06)$ and $81 \%(\mathrm{AOR}, 1.81 ; 95 \% \mathrm{CI}, 1.70,1.93)$ increased risk of death within 5 years of diagnosis. 


\section{Kaplan Meier Estimates}

Overall 5-year survival curves for all women with early-stage breast cancer and according to type of treatment received are shown in Figures $3 \& 4$ with the number at risk in 366 day intervals. Women who received BCS plus RT had significantly lower probability of death at all time points within 5 years of diagnosis, than those who had mastectomy or BCS only. Cohort 3: Guideline-Concordant Care among Women with Stage I, II, \& III Breast Cancer Cohort Characteristics

Among women diagnosed with pathologically staged I, II, and III breast cancer in 2004 to 2009 , the average time to death was $1,246.1$ days $(S D= \pm 600.8)$. The majority of women were diagnosed at age $70-74$ years $(27.1 \%)$, had a comorbidity score $=0(55.7 \%)$, and had a low frequency of PCP visits (55.1\%). More than half of the women were diagnosed at stage I (56.4\%), while about a third were diagnosed at stage II (34.1\%), and the rest at stage III (9.5\%). Most women had ER and PR positive tumors (80.0\% \& 67.3\%) moderately differentiated tumors (44.1\%), tumors $<2 \mathrm{~cm}(38.4 \%)$, and were lymph node negative $(73.1 \%)$. The majority of women lived in areas with a low density of mammography screening (56.2\%) and a high density of oncology treatment centers $(55.0 \%)$. About $80 \%$ were treated by both an oncology and general surgeon. Most, women were white $(88.2 \%)$, lived in metro areas $(83.3 \%)$, areas where $>15 \%$ of the population was college educated (69.5\%), and had an annual income $>\$ 35,000(75.6 \%)$.

\section{Treatment}

Less than $34 \%$ of elderly women with stage I, II, and III breast cancer received GCC. The vast majority of women had the ER (95.0\%) and PR (94.8\%) status of their tumors tested and lymph nodes tested $(92.1 \%)$. Almost all women had some form of primary surgical treatment (99.5\%), while $74.8 \%$ had BCS and $41.7 \%$ had mastectomy. Type of surgery adding up to 
greater than $100 \%$, reflects women who had both BCS and mastectomy. About $61 \%$ of women had RT, while only a quarter (25.1\%) had chemotherapy. Breaking it down further, $20.8 \%$ of the total sample had timely chemotherapy, while $4.3 \%$ didn't have timely chemotherapy.

Group Differences in 5-Year Survival

Most group differences between women who alive or dead 5 years after diagnosis were significant at the $P \leq 0.001$ level, with the exception of density of oncology treatment centers ( $P$ $=0.002)$, had any surgery $(P=0.172)$, and had chemotherapy $(P=0.036)$. Time-to-death was significantly greater among women who were still living 5 years after diagnosis $(M=2,192.3$ days, $S D= \pm 261.0)$, than those who were not $(M=1016.8$ days, $S D= \pm 402.4)(P<0.001)$. Age \& Health

Five-year survival rates decreased with increasing age at diagnosis, such that $93.6 \%$ of women age 66-69 years were alive within 5 years of diagnosis, whereas $76.7 \%$ of women age $\geq$ 80 years were still living. Women with a comorbidity score $=0$, had higher 5 -year survival rates were higher $(90.8 \%)$, than those with a comorbidity score $=1(86.2 \%)$, or score $\geq 2(78.9 \%)$. Women with a lower frequency of PCP visits also had higher 5-year survival rates (89.6\%), than women with a higher frequency of visits (84.9\%).

\section{Clinical Prognostic Factors}

Five-year survival rates decreased with increasing stage at diagnosis. Women diagnosed at stage I had a survival rate of $92.6 \%$, where as those diagnosed at stage II and III had survival rates of $84.5 \%$ and $67.7 \%$, respectively. Similarly, 5-year survival rates decreased with increasing tumor size, such that women with tumors $<1 \mathrm{~cm}$ had a $94.1 \%$ survival rate, while those with tumors $>5 \mathrm{~cm}$ had a 5-year survival rate of $68.1 \%$. Women with ER and PR positive tumors had higher 5-year survival rates $(89.2 \% \& 89.7 \%)$, than those with ER and PR negative 
tumors $(79.4 \% \& 82.7 \%)$. Those with well differentiated tumors had the highest survival rates (92.4\%), while those with poorly differentiated tumors had the lowest (80.6\%). Elderly women who were lymph node negative had a 5-year survival rate of $90.8 \%$, compared to $78.6 \%$ among women who were lymph node positive.

\section{Oncology Care Resources}

Greater 5-year survival rates were observed among women residing in areas with a higher density of mammography screening centers $(88.5 \%)$ and oncology treatment centers $(88.1 \%)$ vs. low density areas $(86.7 \% \& 86.8 \%$, respectively). Women receiving treatment from an oncology surgeon had the highest 5-year survival rates $(89.7 \%)$, followed by those treated by both oncology and general surgeons (88.2\%), and then those treated by a general surgeon only $(82.7 \%)$.

\section{Socio-Demographic Characteristics}

Higher survival rates were observed among white women vs. women of other race (87.9\% vs. $84.8 \%)$, women residing in metro areas vs. non-metro areas (87.9\% vs. $85.3 \%)$, in areas where $\geq 15 \%$ of the population was college education vs. $<15 \%(88.6 \%$ vs. $84.9 \%)$, and areas with an annual income $>\$ 35,000$ vs. $\leq \$ 35,000(88.6 \%$ vs. $84.3 \%)$.

\section{Treatment}

Among women who received GCC, 5-year survival rates were 92.8\%, while they were 84.9\% among women who received GDC. Women who had their ER \& PR status tested (87.7\% \& $87.7 \%)$, than those who did not $(83.9 \%$ \& 84.0\%). Likewise, women who had their lymph nodes tested had higher 5-year survival rates $(88.1 \%)$, than women who did not have them tested (80.5\%). Five-year survival rates were higher among those who had BCS $(90.3 \%)$, compared to those who did not have BCS (79.3\%), while survival rates were higher among those who did not 
have mastectomy (92.0\%), compared to those who had mastectomy (81.2\%). Individuals who had RT had higher 5-year survival rates $(91.1 \%)$, than those who did not have RT $(81.8 \%)$. Women who had timely chemotherapy had higher 5-year survival rates (87.5\%), than women who did not have chemotherapy in the recommended time period $(83.4 \%)$.

Risk of Death within 5 Years of Diagnosis

Age \& Health

Compared to women diagnosed at age 66-69 years, those diagnosed at age 70-74, age 7579, and age > 80 years were $1.17(95 \% \mathrm{CI}, 1.04,1.33), 1.73(95 \% \mathrm{CI}, 1.54,1.95)$, and $2.85(95 \%$ CI, $2.54,3.20$ ) times more likely to die within 5 years of diagnosis. In contrast to women with a comorbidity score $=0$, those with a comorbidity score $=1$ or score $\geq 2$, had an increased risk of death within 5 years of diagnosis by $38 \%$ (AHR, 1.38; 95\% CI, 1.27, 1.50) and 209\% (AHR, 2.09; 95\% CI, 1.92, 2.27). Those with a high frequency of PCP visits had an $18 \%$ increased hazard of death (AHR, 1.18; 95\% CI, 1.10, 1.26), compared to women with a low frequency of visits.

\section{Clinical Prognostic Factors}

Compared to women diagnosed at stage I, those diagnosed at stage III had an $81 \%$ (AHR, $1.81 ; 95 \%$ CI, 1.48, 2.21) increased hazard for death within 5-years of diagnosis. The hazard of death increased with increasing tumor size, with women with tumors $>5 \mathrm{~cm}$ having the greatest risk (AHR, 2.30; 95\% CI, 1.89, 2.80), compared to women with tumors $<1 \mathrm{~cm}$. Women with ER \& PR negative tumors were $1.48(95 \% \mathrm{CI}, 1.32,1.65)$ and $1.13(95 \% \mathrm{CI}, 1.03,1.25)$ times more likely to die within 5 years of diagnosis, than women with ER \& PR positive tumors. Those with poorly differentiated tumor grades were $1.49(95 \% \mathrm{CI}, 1.33,1.67)$ times more likely to die within 5 years of diagnosis, compared to women with well differentiated tumors. Being lymph node 
negative decreased to hazard for death within 5 years of diagnosis by 32\% (AHR, 0.68; 95\% CI, 0.61, 0.76), compared to being lymph node positive.

\section{Oncology Care Resources}

Women who were treated by an oncology surgeon or both an oncology and general surgeon had 26\% (AHR, 0.74; 95\% CI, 0.62, 0.87) and 22\% (AHR, 0.78; 95\% CI, 0.71, 0.85) decreased risk of death, compared to those treated by a general surgeon only. Kaplan Meier Estimates

Figures 5 \& 6 show 5-year survival rates for all women with pathologically staged I, II, and III breast cancer and by receipt of GCC, with the number remaining at risk in 366 day intervals. Women who received GCC had a lower probability of death within 5-years of diagnosis at all time points, than women who received GDC $(P<0.001)$.

\section{Discussion}

Breast cancer mortality has decreased by $34 \%$ from 1990 to 2010 among all populations of women. ${ }^{3}$ Simultaneously, long-term overall survival has increased to $89 \%$ for 5 -year survival among all women diagnosed with breast cancer, and as high as $100 \%$ for women diagnosed at stage I and 99\% for women diagnosed with localized breast cancer (lymph node negative/nonmetastasized). ${ }^{2,3}$ Despite these promising statistics, multiple factors remain associated with differential survival outcomes among elderly with breast cancer. This study began by examining overall 5-year survival among a broadly inclusive cohort of elderly women with stage I, II, and III breast cancer and how the risk of death is associated with age, health, clinical prognostic factors, oncology care resources, and socio-demographic characteristics. A strong association for increased risk of death within 5 years of diagnosis was observed with increasing age, comorbidity, and stage at diagnosis. The next survival analysis addressed the controversy with 
differential type of loco-regional treatment among elderly women with early-stage breast cancer and demonstrated that women who have BCS without RT or mastectomy have a substantially increased risk of death within 5 years of diagnosis, compared to women who have BCS plus RT. Study findings from the first cohort analysis were also confirmed. Examination of the link between treatment patterns and survival was extended in the third cohort analysis to determine the association between receipt of individual tests and treatments and receipt of GCC, among women with pathologically staged I, II, and III breast cancer. This analysis observed that women who did not have RT, chemotherapy, or GCC were at increased risk of death, in addition to the associations observed by the first two analysis. Overall 5-year survival rates observed among cohorts ranged from $82 \%-88 \%$. A more in-depth discussion of these findings follows.

$\underline{\text { Age \& Health }}$

Across all three study cohorts, age, comorbidity, and frequency of PCP visits were of the strongest and most consistent predictors of overall breast cancer survival. In all analyses the hazard of 5-year mortality increased substantially with increasing age. Compared to women age 66-69 years at diagnosis, those who were a $70-74$ had a $17 \%$ to $24 \%$ increased hazard of death, while those who were age $\geq 80$ years had a 3 to 4 fold increased hazard of death. With increasing age, comes increased comorbidity. Some degree of comorbidity was observed in up to $44 \%$ of women. Compared to women no comorbidity, a comorbidity score $=1$ had a $38 \%$ to $49 \%$ increased risk of death while women with comorbidity score $\geq 2$ had as much as $239 \%$ increased risk of death. The increased hazard of death associated with comorbidity was most likely reflected in the increased hazard of death associated with a high frequency of PCP visits. Age and comorbidity are intrinsic risk factors for mortality, regardless of a history of breast cancer. However, the mortality risk associated with age and comorbidity alone may be amplified given a 
history of breast cancer. Moreover, increasing age and comorbidity are associated with receipt of less aggressive treatment that may also increase the risk of mortality. Patnaik and colleagues (2011) observed similar increased 5-year mortality hazard ratios associated with age and comorbidity among elderly women with breast cancer. ${ }^{50}$ Schonberg et al. (2010) also found that age and comorbidity increased the risk breast cancer specific and other-cause mortality, though the effect was less pronounced for breast cancer-specific mortality. ${ }^{51}$

\section{Clinical Prognostic Factors}

Not surprisingly, one of the strongest predictors of overall 5-year survival across all three study samples was stage at diagnosis. Five-year survival rates ranged from $88 \%$ to $93 \%$ for women diagnosed with stage I breast cancer, in contrast to 58\% to $68 \%$ for those diagnosed with stage III breast cancer. What is more, the hazard of death as much as 4 times higher among women diagnosed at stage III, compared to those diagnosed at stage I. Breast cancer prognosis is very good at earlier stages when the cancer remains localized to the breast tissue and has not spread to the lymphatic system or metastasized to the rest of the bodily organs. In fact, Schonberg and colleagues (2011) found that when elderly women are diagnosed at stage 0 or stage I, they are no more likely to die within 10 years of diagnosis than elderly without a history of breast cancer. ${ }^{52}$ In similar fashion, other clinical cancer characteristics directly associated with stage at diagnosis, such as tumor size and lymph node status were associated with overall survival. Increasing tumor size was negatively associated with survival, such that women with tumors $2-5 \mathrm{~cm}$ and $>5 \mathrm{~cm}$ had roughly a two-fold risk of death within 5-years of diagnosis. For women whose cancer had spread into the lymph nodes, the hazard of death increased more than $30 \%$, compared to those who were lymph node negative. A study by Colzani and colleagues (2011) reported a two-fold increase for hazard of death within 5 years of diagnosis among 
women with 1-3 positive lymph nodes, and almost four-fold increase for those with $\geq 4$ positive lymph nodes. ${ }^{53}$ Aside from chemo prevention or prophylactic use of hormone therapy among women at increased risk for breast cancer, the next best approach to maximizing survival is early detection by means of routine mammography screening. Unfortunately, the well-documented decline in routine mammography screening among elderly women decreases the likelihood for early detection. ${ }^{54,55}$ Despite the positive association between age and increased risk, guidelines for mammography screening make no recommendation for routine screening among women age $\geq 75$ years. ${ }^{56}$ Although routine mammography screening among elderly women is associated with earlier stage at diagnosis and better survival, the debate regarding continued screening among women age $\geq 75$ years persists. ${ }^{57}$ The debate revolves around lack of clinical trial based evidence for continued screening among older women, life-expectancy, competing health demands, and harms of false-positive and over diagnosis. ${ }^{58}$

However, some clinical prognostic factors are unaffected by stage at diagnosis. Factors with a biological basis, such as tumor grade and hormone receptor status, are also associated with survival outcomes. Women whose tumors are well-differentiated and are ER/PR positive have greater survival, than women with poorly differentiated and ER/PR negative. ${ }^{53,50}$ Only about a quarter of women in each cohort had well-differentiated tumors. Poorly differentiated tumors increased the hazard of 5-year mortality by about 50\%. Over 3/4 of elderly had ER positive tumors, while $65 \%$ to $67 \%$ had PR positive tumors. Negative ER tumors increased the hazard of 5-year mortality by $33 \%$ to $48 \%$, while negative PR tumors only increased the hazard by $13 \%$ to $19 \%$ among elderly women. Breast cancers that are ER and PR negative are often times more aggressive and fast growing cancers. Since they do not respond to adjuvant hormone therapy, they tend to be more challenging to treat. 


\section{Oncology Care Resources}

Although the risk of death within 5 years of diagnosis was not different based on density of area-level mammography screening and oncology treatment centers, surgeon specialty was associated with risk of death. Women who were treated by an oncology surgeon had a $19 \%$ $26 \%$ decreased risk of 5-year mortality, than women treated by a general surgeon. While women treated by both an oncology and general surgeon had a $22 \%$ - 32\% decreased risk of mortality. Being treated by both specialties might be associated with a lower mortality risk because division of care may be an indication of high patient volume, a factor associated with increased GCC. ${ }^{59}$ Also, having an oncologist participate in the woman's cancer treatment may ensure coordination of guideline recommended care.

\section{$\underline{\text { Socio-Demographic Characteristics }}$}

Women who resided in areas with greater education and income were at somewhat of a decreased risk of death within 5 years of diagnosis, compared to women residing less educated or lower income areas. Specialty health care services are generally more common to in areas with greater education and income. Also, individuals with greater education and income may be more knowledgeable about breast cancer, be more confident navigating the health care system, and have a greater propensity to seek health care services. ${ }^{60-62}$ In contrast with previous studies, an association with black race and poorer survival was not observed in this study. This difference in findings may be due to the way race was categorized by this study (white vs. non-white). Additionally, some of the poorer survival historically observed among women of black race, may have already been adjusted for with other socio-demographic characteristics and access to oncology care. 


\section{$\underline{\text { Treatment Patterns }}$}

The course of breast cancer treatment is most often determined by cancer characteristics and guided by evidence-based clinical treatment guidelines. In some instances, the course of treatment may be modified in consideration of patient preferences or health status. Women with early-stage breast cancer usually have a choice of primary treatment. Breast-conserving surgery followed by RT is equally as effective for long-term survival as mastectomy for women with tumors $<5 \mathrm{~cm} .{ }^{12-15}$ For many women, BCS plus RT is the preferred choice of treatment because of breast preservation. In spite of that, adjuvant treatments such as RT and chemotherapy often have adverse short and long-term side effects that may be intolerable for older women considered to be frail or in poor health, and may be perceived as an unnecessary burden for those with a short life expectancy. ${ }^{28-31}$ In 2004, the NCCN modified guidelines for RT after BCS applicable to a select group of women age $\geq 70$, following results of the CALGB C9343 trial that found overall survival to be equal between women age $>70$ years with early-stage ER positive breast cancer receiving BCS and tamoxifen vs. BCS plus RT and tamoxifen. ${ }^{32,33,63}$ Additionally, NCCN treatment guidelines make no recommendation for or against chemotherapy in women age $>70$ years, when otherwise indicated, but suggest the decision be made on a case-by-case basis considering patient health. However, concern has been raised whether the omission of RT for early-stage ER positive breast cancers when tamoxifen is taken, is actually as effective for overall survival, since there is a small increase in locoregional recurrence among women who omitted RT. Moreover, retrospective studies have shown that a large proportion of elderly women do not receive RT or chemotherapy, raising the question whether more elderly are omitted from these treatments than should be, and consequently jeopardizing survival. 
Among the sample of elderly women with early-stage breast cancer in this study, $55 \%$ had BCS plus RT, 23\% had mastectomy, and 22\% had BCS without RT. Notably, elderly women who were treated with BCS without RT were at almost twice the risk of 5-year mortality, compared to women who were treated with BCS plus RT. Those who were had mastectomy were also 1.80 times more likely to die within 5 years of diagnosis, compared to women treated with BCS plus RT. Findings from the analysis of elderly women with pathologically staged I thru III breast cancer, also indicate a 53\% increased hazard for 5-year mortality among women who did not receive RT, a $16 \%$ increased mortality risk for women who did not have chemotherapy, and a $22 \%$ increased risk of death for who did not receive chemotherapy within a timely manner. Furthermore, women who did not receive GCC had about a $20 \%$ increased risk of death within 5 years of diagnosis, compared to women who received GCC. The observed increased risk of breast cancer specific and overall mortality among women receiving BCS without vs. BCS plus RT, is corroborated by multiple published studies. ${ }^{51,64,65}$ Yood and colleagues (2008) observed that elderly women treated with BCS without vs. BCS plus RT were 1.86 times the risk of 10year overall mortality, a finding strikingly similar to this study. ${ }^{65}$ Additional findings regarding the receipt of RT, chemotherapy, and GCC may be partially explained by the propensity to use less aggressive or lengthy treatments among elderly women or women who are already in poor health or have a short life expectancy. ${ }^{51,66}$ $\underline{\text { Limitations \& Strengths }}$

Several limitations should be kept in mind when interpreting the results of this study. First of all, this study assessed overall survival and not breast-cancer specific survival. While many of the factors examined in this study have been directly and indirectly associated with breast cancer specific mortality, the results of this study should not be generalized to breast 
cancer specific mortality, but overall mortality, which includes both breast cancer specific mortality and all other causes of death. Another limitation is that this study did not look at the association between more race/ethnicity classifications. Survival disparities among black women have been well-document, but was not captured by this study. Additionally, when examining the association between GCC, receipt of individual treatments, and survival, this study did not make allowances for women age $\geq 70$ years to be omitted from treatment, but rather included older women in the definition of GCC to make comparisons in receipt of aggressive treatment between younger and older women. Completion of adjuvant therapies was not assessed, only the initiation of therapy. Also, tumor receptor status was used as a proxy measure of for tumor receptor testing and does not provide any information regarding the initiation of adjuvant hormone therapy when indicated. Additionally, several of socio-demographic characteristics examined were aggregate census level measures, rather than individual level measures. Finally, the SEER individuals within the SEER database have been found to be of higher income, and higher concentrations of racial and ethnic minorities, as compared to the U.S. population. ${ }^{67}$

Despite these limitations, this study holds several strengths that impart credibility to its findings. First and foremost, this study provides a comprehensive description of how numerous factors are associated with overall survival, beginning by examining the relationship between age, health, clinical prognostic factors, oncology care resources, and socio-demographic characteristics and survival among a large inclusive cohort of women with stage I, II, and III breast cancer. Additional analysis examined the associations between various aspects of treatment and survival among more specific cohorts of elderly women with breast cancer. Moreover, this study examined survival outcomes using a large cohorts spanning 8 years of relatively recent data. Algorithms were used to identify receipt of GCC based on the 
recommended course of treatment according to cancer characteristics put forth by the NCCN and ACSO. Despite containing slightly greater proportions of affluent and minority individuals, SEER is widely accepted as representative of the U.S. population, making these results generalizable to the greater population of elderly women with invasive early-stage breast cancer insured by Medicare.

\section{Significance \& Conclusions}

In conclusion, despite the twenty-plus year improvement in breast cancer survival and relatively high survival rates, several factors remain formidable influences on survival. While certain factors with direct effects on survival, such as stage at diagnosis, are modifiable via earlier detection, others are non-modifiable. Non-modifiable factors, such as age at diagnosis, cannot be changed, and may require novel approaches to improving breast cancer survival in relation to increasing age. This is particularly challenging given the tendency for elderly women to be undertreated, a finding in of itself associated with an increased risk of death. However, a factor that is strongly associated with both survival and age, and may be a promising area to target increased survival among all populations, but especially the elderly, is comorbidity. Multiple chronic disease and its burden of illness is a growing epidemic in the US and throughout developing countries, due to increasing obesity rates and lifestyle. The effects of obesity, lifestyle behaviors, and associated chronic disease are far-reaching. While it would not eliminate all incidence and mortality from breast cancer, decreasing the prevalence of chronic disease, by decreasing obesity and improving lifestyle behaviors, would have a meaningful and positive impact on the incidence and survival from breast cancer. 


\section{References}

1. American Cancer Society. Cancer Facts \& Figures 2015. Atlanta: American Cancer Society; 2015. Available at:

http://www.cancer.org/acs/groups/content/@editorial/documents/document/acspc044552.pdf. Last accessed on Jan 19, 2015.

2. American Cancer Society. Breast Cancer Facts \& Figures 2013-2014. Atlanta: American Cancer Society, Inc. 2013. Available at:

http://www.cancer.org/acs/groups/content/@ research/documents/document/acspc042725.pdf. Last accessed on Jan 19, 2015.

3. Howlader N, Noone AM, Krapcho M, Garshell J, Neyman N, Altekruse SF, et al., (eds). SEER Cancer Statistics Review, 1975-2010, National Cancer Institute. Bethesda, MD, http://seer.cancer.gov/csr/1975_2010/, based on November 2012 SEER data submission, posted to the SEER web site, April 2013.

4. Berry D, Cronin K, Plevritis S, Fryback D, Clarke L, Zelen M, et al. Effect of screening and adjuvant therapy on mortality from breast cancer. N Engl J Med. 2005; 353(17): 17841792.

5. Rosenberg J, Chia Y, Plevritis S. The effect of age, race, tumor size, tumor grade, and disease stage on invasive ductal breast cancer survival in the U.S. SEER database. Breast Cancer Res Treat. 2005; 89(1): 47-54.

6. Fisher B, Redmond C, Fisher E, Caplan R. Relative worth of estrogen or progesterone receptor and pathologic characteristics of differentiation as indicators of prognosis in node negative breast cancer patients: findings from National Surgical Adjuvant Breast and Bowel Project Protocol B-06. J Clin Oncol. 1988; 6(7):1076-1087.

7. Hwang E, Lichtensztajn D, Gomez S, Fowble B, Clarke C. Survival after lumpectomy and mastectomy for early-stage invasive breast cancer: the effect of age and hormone receptor status. Cancer. 2013; 119(7): 1402-1411.

8. Tuttle T, Jarosek S, Habermann E, Yee D, Yuan J, Virnig B. Omission of radiation therapy after breast-conserving surgery in the United States. A Population-Based Analysis of Clinicopathologic Factors. Cancer. 2012; 118(8): 2004-2013.

9. Sun S, Hollenbeak C, Leung A. Deviation from the Standard of Care for Early Breast Cancer in the Elderly: What are the Consequences? Ann Surg Oncol. 2014; Dec 17: DOI 10.1245/s10434-014-4290-5 [Epub ahead of print].

10. Giordano S, Duan Z, Kuo Y, Hortobagyi G, Goodwin J. Use and Outcomes of Adjuvant Chemotherapy in Older Women With Breast Cancer. J Clin Oncol. 2006; 24(18): 27502756. 
11. Javid S, Varghese T, Morris A, Porter M, He H, Buchwald D, et al. Guideline-concordant cancer care and survival among American Indian/Alaskan Native patients. Cancer. 2014; 120(14): 2183-2190.

12. Fisher B, Anderson S, Bryant J, Margolese R, Deutsch M, Fisher E, et al. Twenty-year follow-up trial comparing total mastectomy, lumpectomy, and lumpectomy plus irradiation for the treatment of invasive breast cancer. N Engl J Med. 2002; 347(16): 1233-241.

13. Veronesi U, Cascinelli N, Mariani L, Greco M, Saccozzi R, Luini A, et al. Twenty-year follow-up of a randomize study comparing breast-conserving surgery with radical mastectomy for early breast cancer. N Engl J Med. 2002; 347(16): 1227-1232.

14. Litière S, Werutsky G, Fentiman I, Rutgers E, Christiaens M, Limbergen E, et al. Breast conserving therapy versus mastectomy for stage I-II breast cancer: 20 year follow-up of the EORTC 10801 phase 3 randomised trial. Lancet Oncol. 2012; 13(4): 412-19.

15. Simone N, Dan T, Shih J, Smith S, Sciuto L, Lita E, et al. Twenty-five year results of the national cancer institute randomized breast conservation trial. Breast Cancer Res Treat. 2012; 132(1): 197-203.

16. NIH Consensus Conference. Treatment of early-stage breast cancer. JAMA. 1991; 265(3): 391-395.

17. American Cancer Society. Cancer Treatment and Survivorship Facts \& Figures 2014-2015. Atlanta: American Cancer Society; 2014.

18. Dragun A, Huang B, Tucker T, Spanos W. Increasing mastectomy rates among all age groups for early-stage breast cancer: a 10-year study of surgical choice. Breast J. 2012; 18(4): $318-325$.

19. Mahmood U, Hanlon A, Koshy M, Buras R, Chumsri S, Tkaczuk K, et al. Increasing national mastectomy rates for the treatment of early-stage breast cancer. Ann Surg Oncol. 2013; 20(5): 1436-1443.

20. McGuire K, Santillan A, Kaur P, Meade T, Parbhoo J, Mathias M, et al. Are mastectomies on the rise? A 13-year trend analysis of the selection of mastectomy versus breast conservation therapy in 5865 patients. Ann Surg Oncol. 2009; 16(10): 2682-2690.

21. Boscoe F, Johnson C, Henry K, Goldberg D, Shahabi K, Elkin E, et al. Geographic proximity to treatment for early-stage breast cancer and likelihood of mastectomy. Breast. 2011; 20(4): 324-328.

22. Goyal S, Chandwani S, Haffty B, Demissie K. Effect of travel distance and time to radiotherapy on likelihood of receiving mastectomy. Ann Surg Oncol. 2014; Sept 23: Epub ahead of print. 
23. Bradley C, Given C, Baser O, Gardiner J. Influence of surgical and treatment choices on the cost of breast cancer care. Eur J Health Econ. 2003; 4(2): 96-101.

24. Jacobs L, Kelley K, Rosson G, Detrani M, Chang D. Disparities in urban and rural mastectomy populations : the effects of patient- and county-level factors on likelihood of receipt of mastectomy. Ann Surg Oncol. 2008; 15(10): 2644-2652.

25. Schroen A, Brenin D, Kelly M, Knaus W, Slingluff C. Impact of patient distance to radiation therapy on mastectomy use in early-stage breast cancer patients. J Clin Oncol. 2005; 23(28): 7074-7080.

26. Dragun A, Huang B, Tucker T, Spanos W. Disparities in the application of adjuvant radiotherapy after breast-conserving surgery for early-stage breast cancer: impact on overall survival. Cancer. 2011; 117(12): 2590-2598.

27. Bhargava A \& Du X. Racial and socioeconomic disparities in adjuvant chemotherapy for older women with lymph node-positive, operable breast cancer. Cancer. 2009; 115(13): 2999-3008.

28. Nuegut A, Hillyer G, Kushi L, Lamerato L, Nathanson S, Ambrosone C, et al. The Breast Cancer Quality of Care Study (BQUAL): A Multi-Center Study to Determine Causes for Noncompliance with Breast Cancer Adjuvant Therapy. Breast J. 2012; 18(3): 203-213.

29. Yarnold J, Ashton A, Bliss J, Homewood J, Harper C, Hanson J, et al. Fractionation sensitivity and dose response of late adverse effects in the breast after radiotherapy for early breast cancer: long-term results of a randomised trial. Radiother Oncol. 2005; 75(1):9-17.

30. Landoni V, Giordano C, Marsella A, Saracino B, Petrongari M, Ferraro A, et al. Evidence from a breast cancer hypofractionated schedule: late skin toxicity assessed by ultrasound. J Exp Clin Cancer Res. 2013; 32(80).

31. Reinisch M, Minckwitz G, Harbeck N, Janni W, Kümmel S, Kaufmann M, et al. Side Effects of Standard Adjuvant and Neoadjuvant Chemotherapy Regimens According to Age Groups in Primary Breast Cancer. Breast Care. 2013; 8(1): 60-66.

32. Carlson R, Allred D, Anderson B, Burstein H, Carter W, Edge S, et al. Breast cancer. Clinical practice guidelines in oncology. J Natl Compr Canc Netw. 2009; 7(2):122-192.

33. Hughes K, Schnaper L, Berry D, Cirrincione C, McCormick B, Shank B, et al. Lumpectomy plus tamoxifen with or without irradiation in women 70 years of age or older with early breast cancer. N Engl J Med. 2004; 351(10): 971-977.

34. McCormick B, Ottesen R, Hughes M, Javid S, Khan S, Mortimer J, et al. Impact of Guideline Changes on Use or Omission of Radiation in the Elderly with Early Breast Cancer: Practice Patterns at National Comprehensive Cancer Network Institutions. J Am Coll Surg. 2014; 219(4):796-802. 
35. Mackey R, Chandru Kowdley G. Treatment practices and outcomes of elderly women with breast cancer in a community hospital. Am Surg. 2014; 80(7): 714-719.

36. Wheeler S, Carpenter W, Peppercorn J, Schenck A, Weinberger M, Biddle A. Predictors of timing of adjuvant chemotherapy in older women with hormone receptor-negative, stages II-III breast cancer. Breast Cancer Res Treat. 2012; 131(1): 207-216.

37. Martinez S, Shah D, Tseng W, Canter R, Bold R. Rural-urban disparities in use of postlumpectomy radiation. Med Oncol. 2012; 29(5): 3250-3257.

38. Hershman D, Wang X, McBride R, Jacobson J, Grann V, Neugut A. Delay of adjuvant chemotherapy initiation following breast cancer surgery among elderly women. Breast Cancer Res Treat. 2006; 99(3): 313-321.

39. Freedman R, Virgo K, He Y, Pavluck A, Winer E, Ward E, et al. The Association of Race/Ethnicity, Insurance Status, and Socioeconomic Factors With Breast Cancer Care. 2010; 117(1): 180-189.

40. Wu X, Lund M, Kimmick G, Richardson L, Sabatino S, Chen V, et al. Influence of race, insurance, socioeconomic status, and hospital type on receipt of guideline-concordant adjuvant systemic therapy for locoregional breast cancers. J Clin Oncol. 2012; 30(2): $142-150$.

41. Iqbal J, Ginsburg O, Rochon P, Sun $\mathrm{P}^{1}$, Narod S. Differences in breast cancer stage at diagnosis and cancer-specific survival by race and ethnicity in the United States. JAMA. 2015; 313(2): 165-173.

42. Harper S, Lynch J, Meersman S, Breen N, Davis W, Reichman M. Trends in areasocioeconomic and race-ethnic disparities in breast cancer incidence, stage at diagnosis, screening, mortality, and survival among women ages 50 years and over (1987-2005). Cancer Epidemiol Biomarkers Prev. 2009; 18(1): 121-131.

43. Maskarinec G, Sen C, Koga K, Conroy S. Ethnic differences in breast cancer survival: status and determinants. Womens Health (Lond Engl). 2011; 7(6): 677-687.

44. Cunningham J, Butler W. Racial disparities in female breast cancer in South Carolina: clinical evidence for a biological basis. Breast Cancer Res Treat. 2004; 88(2): 161-176.

45. Engels E, Pfeiffer R, Ricker W, Wheeler W, Parsons R, Warren J. Use of Surveillance, Epidemiology, and End Results-Medicare data to conduct case-control studies of cancer among the US elderly. Am J Epidemiol. 2011; 174(7): 860-870.

46. Surveillance epidemiology and end results. SEER-Medicare: About the Data Files.

Available at: http://appliedresearch.cancer.gov/seermedicare/aboutdata/. Last accessed on Oct 29, 2014. 
47. U.S. Health Resources and Services Administration. Bureau of Health Professions. Area Resource File, 2009-2010 Release Rockville, MD : U.S. Department of Health and Human Services. Fairfax, VA : Quality Resource Systems, Inc. 2010; Codebook: HE001(2010).

48. Klabunde C, Potosky A, Legler J, Warren J. Development of a comorbidity index using physician claims data. J Clin Epidemiol. 2000; 53(12): 1258-1267.

49. Charlson M, Pompei P, Ales K, MacKenzie C. A new method of classifying prognostic comorbidity in longitudinal studies: development and validation. J Chronic Dis. 1987; 40(5): 373-383.

50. Patnaik J, Byers T, Diguiseppi C, Denberg T, Dabelea D. The influence of comorbidities on overall survival among older women diagnosed with breast cancer. J Natl Cancer Inst. 2011; 103(14): 1101-1111.

51. Schonberg M, Marcantonio E, Li D, Silliman R, Ngo L, McCarthy E. Breast cancer among the oldest old: tumor characteristics, treatment choices, and survival. J Clin Oncol. 2010; 28(12): 2038-2045.

52. Schonberg M, Marcantonio E, Ngo L, Li D, Silliman R, McCarthy E. Causes of death and relative survival of older women after a breast cancer diagnosis. J Clin Oncol. 2011; 29(12):1570-1577.

53. Colzani E, Liljegren A, Johansson A, Adolfsson J, Hellborg H, Hall P, et al. Prognosis of patients with breast cancer: causes of death and effects of time since diagnosis, age, and tumor characteristics. J Clin Oncol. 2011; 29(30): 4014-4021.

54. Randolph W, Goodwin J, Mahnken J, Freeman J. Regular mammography use is associated with elimination of age-related disparities in size and stage of breast cancer at diagnosis. Ann Intern Med. 2002; 137(10): 783-790.

55. Vacek P, Skelly J. A prospective study of the use and effects of screening mammography in women aged 70 and older. J Am Geriatr Soc. 2015; 63(1): 1-7.

56. US Preventive Services Task Force. Screening for breast cancer: U.S. Preventive Services Task Force recommendation statement. Ann Intern Med. 2009; 151(10): 716-726, W236.

57. Vyas A, Madhavan S, Sambamoorthi U. Association between persistence with mammography screening and stage at diagnosis among elderly women diagnosed with breast cancer. Breast Cancer Res Treat. 2014; 148(3): 645-654.

58. Braithwaite D, Mandelblatt J, Kerlikowske K. To screen or not to screen older women for breast cancer: a conundrum. Future Oncol. 2013; 9(6): 763-766. 
59. Kimmick G, Camacho F, Mackley H, Kern T, Yao N, Mathews S, et al. Individual, Area, and Provider Characteristics Associated With Care Received for Stages I to III Breast Cancer in a Multistate Region of Appalachia. J Oncol Pract. 2014; Sep 16. pii: JOP.2014.001397. [Epub ahead of print].

60. Freedman R, Kouri E, West D, Keating N. Racial/Ethnic Disparities in Knowledge About One's Breast Cancer Characteristics. Cancer. 2015; Jan 26. doi: 10.1002/cncr.28977. [Epub ahead of print].

61. Ko N, Darnell J, Calhoun E, Freund K, Wells K, Shapiro C, et al. Can Patient Navigation Improve Receipt of Recommended Breast Cancer Care? Evidence From the National Patient Navigation Research Program. Clin Oncol. 2014; 32(25): 2758-2764.

62. Freund K, Battaglia T, Calhoun E, Darnell J, Dudley D, Fiscella K, et al. Impact of Patient Navigation on Timely Cancer Care: The Patient Navigation Research Program. J Natl Cancer Inst. 2014; 106(6): dju115. doi: 10.1093/jnci/dju115.

63. Hughes K, Schnaper L, Bellon J, Cirrincione C, Berry D, McCormick B, et al. Lumpectomy plus tamoxifen with or without irradiation in women age 70 years or older with early breast cancer: long-term follow-up of CALGB 9343. J Clin Oncol. 2013; 31(19): 23822387.

64. Sun S, Hollenbeak C, Leung A. Deviation from the Standard of Care for Early Breast Cancer in the Elderly: What are the Consequences? Ann Surg Oncol. 2014; Dec 17: DOI 10.1245/s10434-014-4290-5 [Epub ahead of print].

65. Yood M, Owusu C, Buist D, Geiger A, Field T, Thwin S, et al. Mortality impact of lessthan-standard therapy in older breast cancer patients. J Am Coll Surg. 2008; 206(1):6675 .

66. Schonberg M, Marcantonio E, Ngo L, Silliman R, McCarthy E. Does Life Expectancy Affect Treatment of Women Aged 80 and Older with Early Stage Breast Cancers? J Geriatr Oncol. 2012; 3(1): 8-16.

67. Warren J, Klabunde C, Schrag D, Bach P, Riley G. Overview of the SEER-Medicare data: content, research applications, and generalizability to the United States elderly population. Med Care. 2002; 40(8 Suppl):IV,3-18. 
Table 1

Characteristics of Elderly Women with Invasive Non-Metastatic Breast Cancer

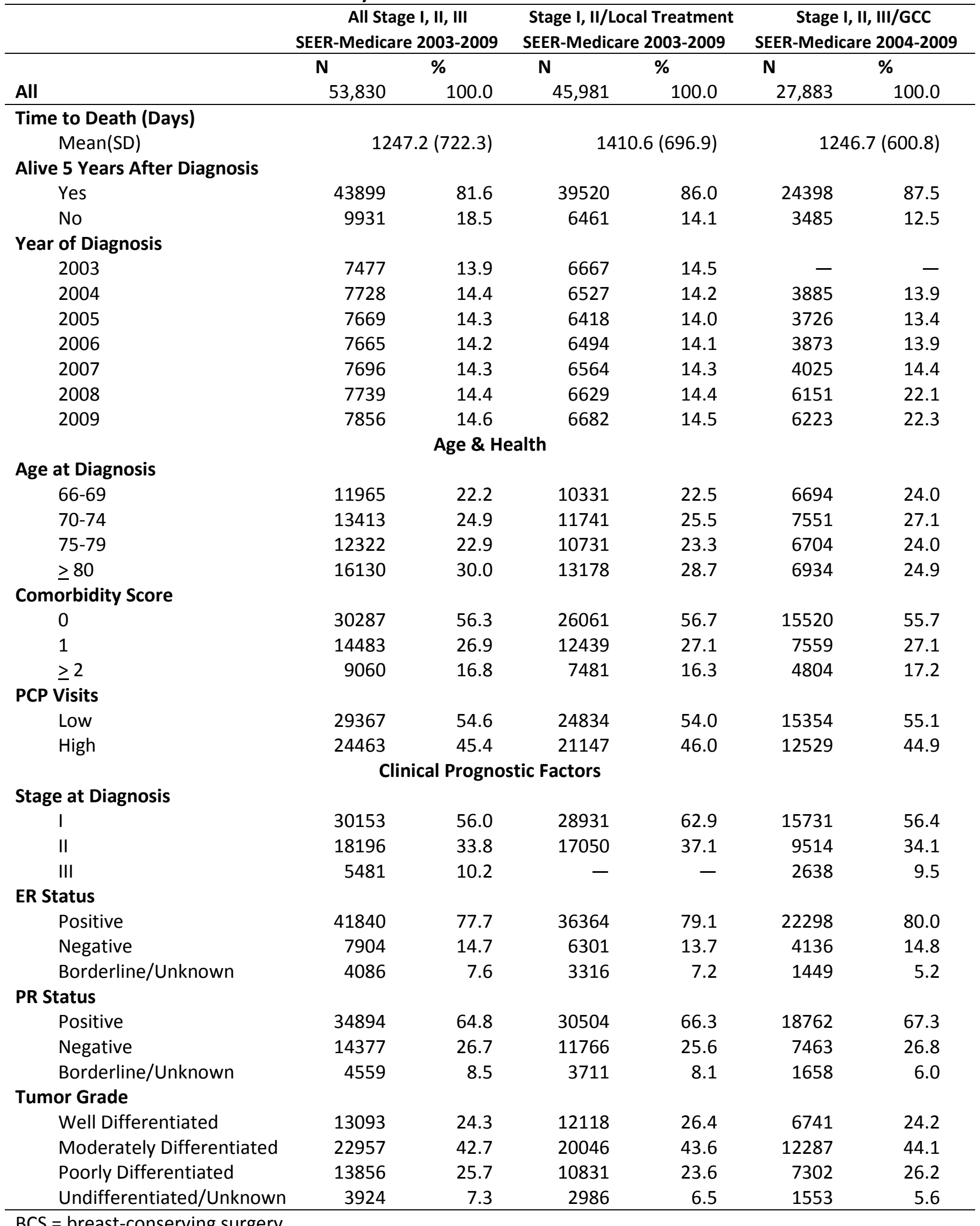


Table 1

Characteristics of Elderly Women with Invasive Non-Metastatic Breast Cancer

\begin{tabular}{|c|c|c|c|c|c|c|}
\hline & \multicolumn{2}{|c|}{$\begin{array}{c}\text { All Stage I, II, III } \\
\text { SEER-Medicare 2003-2009 }\end{array}$} & \multicolumn{2}{|c|}{$\begin{array}{l}\text { Stage I, II/Local Treatment } \\
\text { SEER-Medicare 2003-2009 }\end{array}$} & \multicolumn{2}{|c|}{$\begin{array}{c}\text { Stage I, II, III/GCC } \\
\text { SEER-Medicare 2004-2009 }\end{array}$} \\
\hline & $\mathbf{N}$ & $\%$ & $\mathbf{N}$ & $\%$ & $\mathbf{N}$ & $\%$ \\
\hline All & 53,830 & 100.0 & 45,981 & 100.0 & 27,883 & 100.0 \\
\hline
\end{tabular}

Tumor Size

$<1 \mathrm{~cm}$

$<2 \mathrm{~cm}$

$2-5 \mathrm{~cm}$

$>5 \mathrm{~cm}$

Lymph Nodes

Positive

Negative

Mammography Screening Centers

Low

High

Oncology Treatment Centers

Low

High

Type of Surgeon Seen

General Only

Oncology Only

Both

Race

White

Other

Education

$<15 \%$ college degree

$\geq 15 \%$ college degree

Annual Income

$\leq \$ 35,000$

$>\$ 35,000$

Metro Status

Non-metro

Metro

Loco-Regional Treatment

BCS+Radiation

Mastectomy

BCS Only

Guideline-Concordant Care

Yes

No

ER Status Tested

Yes

No

$\begin{array}{rrrrrr}- & - & - & - & 8201 & 29.4 \\ - & - & - & - & 10701 & 38.4 \\ - & - & - & - & 7834 & 28.1 \\ - & - & - & - & 1147 & 4.1 \\ & & & & & \\ - & - & - & - & 7505 & 26.9 \\ - & - & - & - & 20378 & 73.1\end{array}$

27783

26047

30146

23684

9613

3235

40001

Socio-Demographic Characteristics

47698

6132

16232

37598

13057

40773

8965

44865

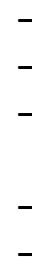

-

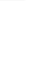

$-$

$-$

51.6

48.4

23789

22192

56.0

44.0

25761

20220

18.2

6.1

8555

2644

34782

75.7

88.6

11.4

41090

4891

30.2

69.9

13557

32424

24.3

75.7

35128

16.7

83.4

7686

38295

Tests \& Treatments
10853

\begin{tabular}{|c|c|c|c|c|}
\hline- & 25067 & 54.5 & - & - \\
\hline- & 10768 & 23.4 & - & - \\
\hline- & 10146 & 22.1 & - & - \\
\hline- & - & - & 9336 & 33.5 \\
\hline- & - & - & 18547 & 66.5 \\
\hline- & - & - & 26482 & 95.0 \\
\hline- & - & - & 1401 & 5.02 \\
\hline
\end{tabular}

56.2

43.8

45.0

55.0

14.5

5.9

79.6

22200

24594

88.2

11.8

30.5

69.5

24.4

75.6

16.7

83.3

23223
5.02 
Table 1

Characteristics of Elderly Women with Invasive Non-Metastatic Breast Cancer

\begin{tabular}{|c|c|c|c|c|c|c|}
\hline & \multicolumn{2}{|c|}{$\begin{array}{c}\text { All Stage I, II, III } \\
\text { SEER-Medicare 2003-2009 }\end{array}$} & \multicolumn{2}{|c|}{$\begin{array}{l}\text { Stage I, II/Local Treatment } \\
\text { SEER-Medicare 2003-2009 }\end{array}$} & \multicolumn{2}{|c|}{$\begin{array}{c}\text { Stage I, II, III/GCC } \\
\text { SEER-Medicare 2004-2009 }\end{array}$} \\
\hline & $\mathbf{N}$ & $\%$ & $\mathbf{N}$ & $\%$ & $\mathbf{N}$ & $\%$ \\
\hline All & 53,830 & 100.0 & 45,981 & 100.0 & 27,883 & 100.0 \\
\hline \multicolumn{7}{|l|}{ PR Status Tested } \\
\hline Yes & - & - & - & - & 26435 & 94.8 \\
\hline No & - & - & - & - & 1448 & 5.19 \\
\hline \multicolumn{7}{|c|}{ BCS = Breast-Conserving Surgery } \\
\hline \multicolumn{7}{|l|}{ Lymph Nodes Tested } \\
\hline Yes & - & - & 一 & - & 25685 & 92.1 \\
\hline No & - & - & - & - & 2198 & 7.9 \\
\hline \multicolumn{7}{|l|}{ Had any Surgery } \\
\hline Yes & - & - & - & - & 27754 & 99.5 \\
\hline No & - & - & - & - & 129 & 0.5 \\
\hline \multicolumn{7}{|l|}{ Had BCS } \\
\hline Yes & - & - & 一 & - & 20865 & 74.8 \\
\hline No & - & - & - & - & 7018 & 25.2 \\
\hline \multicolumn{7}{|l|}{ Had Mastectomy } \\
\hline Yes & - & - & 一 & - & 11636 & 41.7 \\
\hline No & - & - & - & - & 16247 & 58.3 \\
\hline \multicolumn{7}{|l|}{ Had Radiation } \\
\hline Yes & - & - & 一 & - & 17023 & 61.1 \\
\hline No & - & - & - & - & 10860 & 39.0 \\
\hline \multicolumn{7}{|l|}{ Had Chemotherapy } \\
\hline Yes & - & - & - & - & 6992 & 25.1 \\
\hline No & - & - & - & - & 20891 & 74.9 \\
\hline \multicolumn{7}{|l|}{ Time to Chemotherapy } \\
\hline Appropriate & - & - & - & - & 5796 & 20.8 \\
\hline Not Appropriate & - & - & - & - & 1196 & 4.3 \\
\hline No Chemotherapy & - & - & - & - & 20891 & 74.9 \\
\hline
\end{tabular}

$\mathrm{BCS}=$ breast-conserving surgery 
Table 2

Comparison of Characteristics Between Elderly Women Alive and Dead 5 Years After Invasive Non-Metastatic Breast Cancer Diagnosis

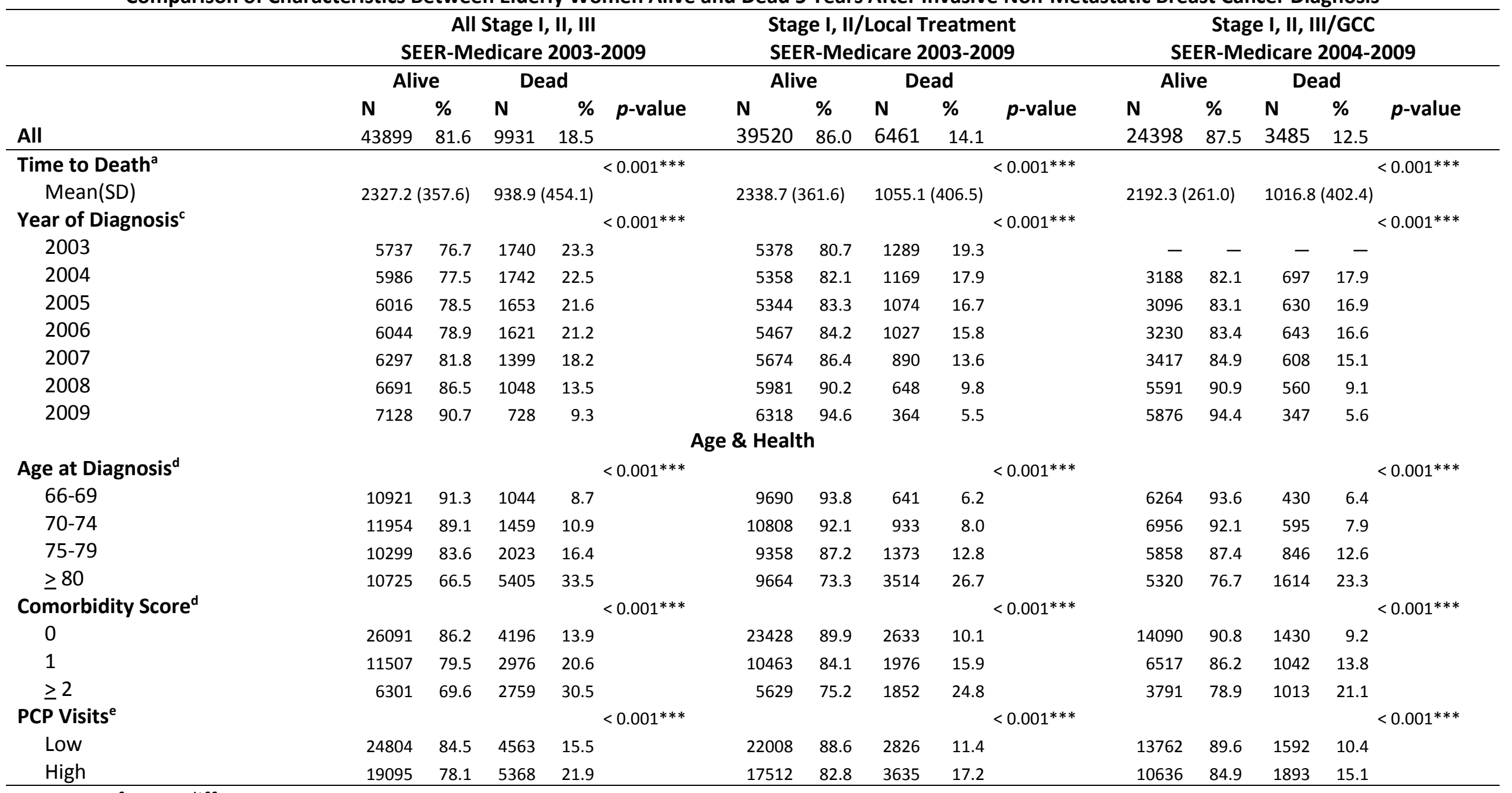

$\mathrm{a}=\mathrm{t}$-test of mean differences

$\mathrm{b}=\mathrm{cmh}$ chi square test of general association

$\mathrm{c}=\mathrm{cmh}$ chi square test of location shift using table scores

$\mathrm{d}=\mathrm{cmh}$ chi square test of location shift using modified ridit scores

$\mathrm{e}=$ Pearson's chi square test of association

$* \mathrm{p}<0.05 ; * * \mathrm{p}<0.01 ; * * * \mathrm{p}<0.001$

$\mathrm{BCS}=$ Breast-Conserving Surgery 
Table 2

Comparison of Characteristics Between Elderly Women Alive and Dead 5 Years After Invasive Non-Metastatic Breast Cancer Diagnosis

\begin{tabular}{|c|c|c|c|c|c|c|c|c|c|c|c|c|c|c|c|}
\hline & \multicolumn{5}{|c|}{$\begin{array}{c}\text { All Stage I, II, III } \\
\text { SEER-Medicare 2003-2009 }\end{array}$} & \multicolumn{5}{|c|}{$\begin{array}{l}\text { Stage I, II/Local Treatment } \\
\text { SEER-Medicare 2003-2009 }\end{array}$} & \multicolumn{5}{|c|}{$\begin{array}{c}\text { Stage I, II, III/GCC } \\
\text { SEER-Medicare 2004-2009 }\end{array}$} \\
\hline & \multicolumn{2}{|c|}{ Alive } & \multicolumn{2}{|c|}{ Dead } & \multirow[b]{2}{*}{$p$-value } & \multicolumn{2}{|c|}{ Alive } & \multicolumn{2}{|c|}{ Dead } & \multirow[b]{2}{*}{$p$-value } & \multicolumn{2}{|c|}{ Alive } & \multicolumn{2}{|c|}{ Dead } & \multirow[b]{2}{*}{$p$-value } \\
\hline & $\mathbf{N}$ & $\%$ & $\mathbf{N}$ & $\%$ & & $\mathbf{N}$ & $\%$ & $\mathbf{N}$ & $\%$ & & $\mathbf{N}$ & $\%$ & $\mathbf{N}$ & $\%$ & \\
\hline All & 43899 & 81.6 & 9931 & 18.5 & & 39520 & 86.0 & 6461 & 14.1 & & 24398 & 87.5 & 3485 & 12.5 & \\
\hline \multicolumn{16}{|c|}{ Clinical Prognostic Factors } \\
\hline Stage at Diagnosis ${ }^{d}$ & & & & & $<0.001 * * *$ & & & & & $<0.001 * * *$ & & & & & $<0.001 * * *$ \\
\hline II & 14085 & 77.4 & 4111 & 22.6 & & 13623 & 79.9 & 3427 & 20.1 & & 8038 & 84.5 & 1476 & 15.5 & \\
\hline III & 3182 & 58.1 & 2299 & 41.9 & & - & - & - & - & & 1787 & 67.7 & 851 & 32.3 & \\
\hline ER Status $^{\text {b }}$ & & & & & $<0.001 * * *$ & & & & & $<0.001 * * *$ & & & & & $<0.001 * * *$ \\
\hline Positive & 35197 & 84.1 & 6643 & 15.9 & & 31842 & 87.6 & 4522 & 12.4 & & 19897 & 89.2 & 2401 & 10.8 & \\
\hline Negative & 5687 & 72.0 & 2217 & 28.1 & & 4982 & 79.1 & 1319 & 20.9 & & 3282 & 79.4 & 854 & 20.7 & \\
\hline Borderline/Unknown & 3015 & 73.8 & 1071 & 26.2 & & 2696 & 81.3 & 620 & 18.7 & & 1219 & 84.1 & 230 & 15.9 & \\
\hline PR Status $^{\text {b }}$ & & & & & $<0.001^{* * *}$ & & & & & $<0.001 * * *$ & & & & & $<0.001 * * *$ \\
\hline Negative & 10894 & 75.8 & 3483 & 24.2 & & 9633 & 81.9 & 2133 & 18.1 & & 6174 & 82.7 & 1289 & 17.3 & \\
\hline Borderline/Unknown & 3388 & 74.3 & 1171 & 25.7 & & 3027 & 81.6 & 684 & 18.4 & & 1401 & 84.5 & 257 & 15.5 & \\
\hline Tumor Grade & & & & & $<0.001^{* * *}$ & & & & & $<0.001^{* * *}$ & & & & & $<0.001 * * *$ \\
\hline Well Differentiated & 11537 & 88.1 & 1556 & 11.9 & & 10891 & 89.9 & 1227 & 10.1 & & 6228 & 92.4 & 513 & 7.6 & \\
\hline Moderately Differentiated & 19231 & 83.8 & 3726 & 16.2 & & 17409 & 86.9 & 2637 & 13.2 & & 10945 & 89.1 & 1342 & 10.9 & \\
\hline Poorly Differentiated & 10152 & 73.3 & 3704 & 26.7 & & 8681 & 80.2 & 2150 & 19.9 & & 5882 & 80.6 & 1420 & 19.5 & \\
\hline Undifferentiated/Unknown & 2979 & 75.9 & 945 & 24.1 & & 2539 & 85.0 & 447 & 15.0 & & 1343 & 86.5 & 210 & 13.5 & \\
\hline Tumor Size $^{d}$ & & & & & & & & & & & & & & & $<0.001 * * *$ \\
\hline$<1 \mathrm{~cm}$ & - & - & - & - & & - & - & - & - & & 7720 & 94.1 & 481 & 5.9 & \\
\hline$<2 \mathrm{~cm}$ & - & - & - & - & & - & - & - & - & & 9610 & 89.8 & 1091 & 10.2 & \\
\hline $2-5 \mathrm{~cm}$ & - & - & - & - & & - & - & - & - & & 6287 & 80.3 & 1547 & 5.6 & \\
\hline$>5 \mathrm{~cm}$ & - & - & - & - & & - & - & - & - & & 781 & 68.1 & 366 & 31.9 & \\
\hline
\end{tabular}

$\mathrm{a}=\mathrm{t}$-test of mean differences

$\mathrm{b}=\mathrm{cmh}$ chi square test of general association

$\mathrm{c}=\mathrm{cmh}$ chi square test of location shift using table scores

$\mathrm{d}=\mathrm{cmh}$ chi square test of location shift using modified ridit scores

$\mathrm{e}=$ Pearson's chi square test of association

$* \mathrm{p}<0.05 ; * * \mathrm{p}<0.01 ; * * * \mathrm{p}<$

0.001

BCS = Breast-Conserving Surgery 
Table 2

Comparison of Characteristics Between Elderly Women Alive and Dead 5 Years After Invasive Non-Metastatic Breast Cancer Diagnosis

\begin{tabular}{|c|c|c|c|c|c|c|c|c|c|c|c|c|c|c|c|}
\hline & \multicolumn{5}{|c|}{$\begin{array}{c}\text { All Stage I, II, III } \\
\text { SEER-Medicare 2003-2009 }\end{array}$} & \multicolumn{5}{|c|}{$\begin{array}{l}\text { Stage I, II/Local Treatment } \\
\text { SEER-Medicare 2003-2009 }\end{array}$} & \multicolumn{5}{|c|}{$\begin{array}{c}\text { Stage I, II, III/GCC } \\
\text { SEER-Medicare 2004-2009 }\end{array}$} \\
\hline & \multicolumn{2}{|c|}{ Alive } & \multicolumn{2}{|c|}{ Dead } & \multirow[b]{2}{*}{$p$-value } & \multicolumn{2}{|c|}{ Alive } & \multicolumn{2}{|c|}{ Dead } & \multirow{3}{*}{$p$-value } & \multicolumn{2}{|c|}{ Alive } & \multicolumn{2}{|c|}{ Dead } & \multirow{3}{*}{$p$-value } \\
\hline & $\mathbf{N}$ & $\%$ & $\mathbf{N}$ & $\%$ & & $\mathbf{N}$ & $\%$ & $\mathbf{N}$ & $\%$ & & $\mathbf{N}$ & $\%$ & $\mathbf{N}$ & $\%$ & \\
\hline All & 43899 & 81.6 & 9931 & 18.5 & & 39520 & 86.0 & 6461 & 14.1 & & 24398 & 87.5 & 3485 & 12.5 & \\
\hline Lymph Nodes $^{\mathrm{e}}$ & & & & & & & & & & & & & & & $<0.001 * * *$ \\
\hline Positive & - & - & - & - & & - & - & - & - & & 5897 & 78.6 & 1877 & 9.2 & \\
\hline Negative & - & - & - & - & & - & - & - & - & & 18501 & 90.8 & 1608 & 21.4 & \\
\hline \multicolumn{16}{|c|}{ Oncology Care Resources } \\
\hline Mammography Screening Centers ${ }^{\mathrm{e}}$ & & & & & $0.001 * * *$ & & & & & $<0.001 * * *$ & & & & & $<0.001 * * *$ \\
\hline Low & 22514 & 81.0 & 5269 & 19.0 & & 20317 & 85.4 & 3472 & 14.6 & & 13588 & 86.7 & 2077 & 13.3 & \\
\hline High & 21385 & 82.1 & 4662 & 17.9 & & 19203 & 86.5 & 2989 & 13.5 & & 10810 & 88.5 & 1408 & 11.5 & \\
\hline Oncology Treatment Centers ${ }^{e}$ & & & & & $<0.001 * * *$ & & & & & $<0.001 * * *$ & & & & & $0.002 * *$ \\
\hline Low & 24394 & 80.9 & 5752 & 19.1 & & 21995 & 85.4 & 3766 & 14.6 & & 10890 & 86.8 & 1651 & 13.2 & \\
\hline High & 19505 & 82.4 & 4179 & 17.6 & & 17525 & 86.7 & 2695 & 13.3 & & 13508 & 88.1 & 1834 & 12.0 & \\
\hline General Only & 7062 & 73.5 & 2551 & 26.5 & & 6675 & 78.0 & 1880 & 22.0 & & 3344 & 82.7 & 701 & 17.3 & \\
\hline Oncology Only & 2664 & 82.4 & 571 & 17.7 & & 2307 & 87.3 & 337 & 12.8 & & 1469 & 89.7 & 169 & 10.3 & \\
\hline Both & 33444 & 83.6 & 6557 & 16.4 & & 30538 & 87.8 & 4244 & 12.2 & & 19585 & 88.2 & 2615 & 11.8 & \\
\hline \multicolumn{16}{|c|}{ Socio-Demographic Characteristics } \\
\hline Race $^{e}$ & & & & & $<0.001 * * *$ & & & & & 0.092 & & & & & $<0.001 * * *$ \\
\hline White & 39028 & 81.8 & 8670 & 18.2 & & 35355 & 86.0 & 5735 & 14.0 & & 21609 & 87.9 & 2985 & 12.1 & \\
\hline Other & 4871 & 79.4 & 1261 & 20.6 & & 4165 & 85.2 & 726 & 14.8 & & 2789 & 84.8 & 500 & 15.2 & \\
\hline Education $^{\mathrm{e}}$ & & & & & $<0.001 * * *$ & & & & & $<0.001 * * *$ & & & & & $<0.001 * * *$ \\
\hline$<15 \%$ college degree & 12752 & 78.6 & 3480 & 21.4 & & 11315 & 83.5 & 2242 & 16.5 & & 7211 & 84.9 & 1282 & 15.1 & \\
\hline$\geq 15 \%$ college degree & 31147 & 82.8 & 6451 & 17.2 & & 28205 & 87.0 & 4219 & 13.0 & & 17187 & 88.6 & 2203 & 11.4 & \\
\hline Annual Income & & & & & $<0.001 * * *$ & & & & & $<0.001^{* * *}$ & & & & & $<0.001^{* * *}$ \\
\hline$\leq \$ 35,000$ & 10085 & 77.2 & 2972 & 22.8 & & 8962 & 82.6 & 1891 & 17.4 & & 5734 & 84.3 & 1072 & 15.8 & \\
\hline$>\$ 35,000$ & 33814 & 82.9 & 6959 & 17.1 & & 30558 & 87.0 & 4570 & 13.01 & & 18664 & 88.6 & 2413 & 11.5 & \\
\hline
\end{tabular}

$\mathrm{a}=\mathrm{t}$-test of mean differences

$\mathrm{b}=\mathrm{cmh}$ chi square test of general association

$\mathrm{c}=\mathrm{cmh}$ chi square test of location shift using table scores

$\mathrm{d}=\mathrm{cmh}$ chi square test of location shift using modified ridit scores

$\mathrm{e}=$ Pearson's chi square test of association

$* \mathrm{p}<0.05 ; * * \mathrm{p}<0.01 ; * * * \mathrm{p}<0.001$

$\mathrm{BCS}=$ Breast-Conserving Surgery 
Table 2

Comparison of Characteristics Between Elderly Women Alive and Dead 5 Years After Invasive Non-Metastatic Breast Cancer Diagnosis

\begin{tabular}{|c|c|c|c|c|c|c|c|c|c|c|c|c|c|c|c|}
\hline & \multicolumn{5}{|c|}{$\begin{array}{c}\text { All Stage I, II, III } \\
\text { SEER-Medicare 2003-2009 }\end{array}$} & \multicolumn{5}{|c|}{$\begin{array}{l}\text { Stage I, II/Local Treatment } \\
\text { SEER-Medicare 2003-2009 }\end{array}$} & \multicolumn{5}{|c|}{$\begin{array}{c}\text { Stage I, II, III/GCC } \\
\text { SEER-Medicare 2004-2009 }\end{array}$} \\
\hline & \multicolumn{2}{|c|}{ Alive } & \multicolumn{2}{|c|}{ Dead } & \multirow[b]{2}{*}{$p$-value } & \multicolumn{2}{|c|}{ Alive } & \multicolumn{2}{|c|}{ Dead } & \multirow[b]{2}{*}{$p$-value } & \multicolumn{2}{|c|}{ Alive } & \multicolumn{2}{|c|}{ Dead } & \multirow{3}{*}{$p$-value } \\
\hline & $\mathbf{N}$ & $\%$ & $\mathbf{N}$ & $\%$ & & $\mathbf{N}$ & $\%$ & $\mathbf{N}$ & $\%$ & & $\mathbf{N}$ & $\%$ & $\mathbf{N}$ & $\%$ & \\
\hline All & 43899 & 81.6 & 9931 & 18.5 & & 39520 & 86.0 & 6461 & 14.1 & & 24398 & 87.5 & 3485 & 12.5 & \\
\hline Metro Status $^{\mathrm{e}}$ & & & & & $<0.001^{* * *}$ & & & & & $<0.001^{* * *}$ & & & & & $<0.001^{* * *}$ \\
\hline Non-metro & 7134 & 79.6 & 1831 & 20.4 & & 6477 & 84.3 & 1209 & 15.7 & & 3975 & 85.3 & 685 & 14.7 & \\
\hline Metro & 36765 & 82.0 & 8100 & 18.1 & & 33043 & 86.3 & 5252 & 13.7 & & 20423 & 87.9 & 2800 & 12.1 & \\
\hline \multicolumn{16}{|c|}{ Tests \& Treatments } \\
\hline Loco-Regional Treatment $^{\mathrm{b}}$ & & & & & & & & & & $<0.001^{* * *}$ & & & & & \\
\hline BCS+Radiation & - & - & - & - & & 23043 & 91.9 & 2024 & 8.1 & & - & - & - & - & \\
\hline Mastectomy & - & - & - & - & & 8541 & 79.3 & 2227 & 20.7 & & - & - & - & - & \\
\hline BCS Only & - & - & - & - & & 7936 & 78.2 & 2210 & 21.8 & & - & - & - & - & \\
\hline Guideline-Concordant Care & & & & & & & & & & & & & & & $<0.001^{* * *}$ \\
\hline Yes & - & - & - & - & & - & - & - & - & & 8659 & 92.8 & 677 & 7.3 & \\
\hline No & - & - & - & - & & - & - & - & - & & 15739 & 84.9 & 2808 & 15.1 & \\
\hline ER Status Tested & & & & & & & & & & & & & & & $<0.001 * * *$ \\
\hline Yes & - & - & - & - & & - & - & - & - & & 23222 & 87.7 & 3260 & 12.3 & \\
\hline No & - & - & - & - & & - & - & - & - & & 1176 & 83.9 & 225 & 16.1 & \\
\hline PR Status Tested & & & & & & & & & & & & & & & $<0.001^{* * *}$ \\
\hline Yes & - & - & - & - & & - & - & - & - & & 23182 & 87.7 & 3253 & 12.3 & \\
\hline No & - & - & - & - & & - & - & - & - & & 1216 & 84.0 & 232 & 16.0 & \\
\hline Lymph Nodes Tested & & & & & & & & & & & & & & & $<0.001^{* * *}$ \\
\hline Yes & - & - & - & - & & - & - & - & - & & 22629 & 88.1 & 3056 & 11.9 & \\
\hline No & - & - & - & - & & - & - & - & - & & 1769 & 80.5 & 429 & 19.5 & \\
\hline Had any Surgery & & & & & & & & & & & & & & & 0.172 \\
\hline Yes & - & - & - & - & & - & - & - & - & & 24280 & 87.5 & 3474 & 12.5 & \\
\hline No & - & - & - & - & & - & - & - & - & & 118 & 91.5 & 11 & 8.5 & \\
\hline
\end{tabular}

$\mathrm{a}=\mathrm{t}$-test of mean differences

$\mathrm{b}=\mathrm{cmh}$ chi square test of general association

$\mathrm{c}=\mathrm{cmh}$ chi square test of location shift using table scores

$\mathrm{d}=\mathrm{cmh}$ chi square test of location shift using modified ridit scores

$\mathrm{e}=$ Pearson's chi square test of association

${ }^{*} \mathrm{p}<0.05$; $^{* *} \mathrm{p}<0.01 ;{ }^{* * *} \mathrm{p}<0.001$

$\mathrm{BCS}=$ Breast-Conserving Surgery 
Table 2

Comparison of Characteristics Between Elderly Women Alive and Dead 5 Years After Invasive Non-Metastatic Breast Cancer Diagnosis

\begin{tabular}{|c|c|c|c|c|c|c|c|c|c|c|c|c|c|c|c|}
\hline & \multicolumn{5}{|c|}{$\begin{array}{c}\text { All Stage I, II, III } \\
\text { SEER-Medicare 2003-2009 }\end{array}$} & \multicolumn{5}{|c|}{$\begin{array}{l}\text { Stage I, II/Local Treatment } \\
\text { SEER-Medicare 2003-2009 }\end{array}$} & \multicolumn{5}{|c|}{$\begin{array}{c}\text { Stage I, II, III/GCC } \\
\text { SEER-Medicare 2004-2009 }\end{array}$} \\
\hline & \multicolumn{2}{|c|}{ Alive } & \multicolumn{2}{|c|}{ Dead } & \multirow[b]{2}{*}{$p$-value } & \multicolumn{2}{|c|}{ Alive } & \multicolumn{2}{|c|}{ Dead } & \multirow{3}{*}{$p$-value } & \multicolumn{2}{|c|}{ Alive } & \multicolumn{2}{|c|}{ Dead } & \multirow{3}{*}{$p$-value } \\
\hline & $\mathbf{N}$ & $\%$ & $\mathbf{N}$ & $\%$ & & $\mathbf{N}$ & $\%$ & $\mathbf{N}$ & $\%$ & & $\mathbf{N}$ & $\%$ & $\mathbf{N}$ & $\%$ & \\
\hline All & 43899 & 81.6 & 9931 & 18.5 & & 39520 & 86.0 & 6461 & 14.1 & & 24398 & 87.5 & 3485 & 12.5 & \\
\hline $\mathrm{Had} \mathrm{BCS}^{\mathrm{e}}$ & & & & & & & & & & & & & & & $<0.001^{* * *}$ \\
\hline Yes & - & - & - & - & & - & - & - & - & & 18830 & 90.3 & 2035 & 9.8 & \\
\hline No & - & - & - & - & & - & - & - & - & & 5568 & 79.3 & 1450 & 20.7 & \\
\hline Had Mastectomy & & & & & & & & & & & & & & & $<0.001^{* * *}$ \\
\hline Yes & - & - & - & - & & - & - & - & - & & 9446 & 81.2 & 2190 & 18.8 & \\
\hline No & - & - & - & - & & - & - & - & - & & 14952 & 92.0 & 1295 & 8.0 & \\
\hline Had Radiation ${ }^{\mathrm{e}}$ & & & & & & & & & & & & & & & $<0.001^{* * *}$ \\
\hline Yes & - & - & - & - & & - & - & - & - & & 15514 & 91.1 & 1509 & 8.9 & \\
\hline No & - & - & - & - & & - & - & - & - & & 8884 & 81.8 & 1976 & 18.2 & \\
\hline Had Chemotherapy ${ }^{\mathrm{e}}$ & & & & & & & & & & & & & & & $0.036 *$ \\
\hline Yes & - & - & - & - & & - & - & - & - & & 6068 & 86.8 & 924 & 13.2 & \\
\hline No & - & - & - & - & & - & - & - & - & & 18330 & 87.7 & 2561 & 12.3 & \\
\hline Time to Chemotherapy ${ }^{b}$ & & & & & & & & & & & & & & & $<0.001 * * *$ \\
\hline Appropriate & - & - & - & - & & - & - & - & - & & 5070 & 87.5 & 726 & 12.5 & \\
\hline Not Appropriate & - & - & - & - & & - & - & - & - & & 998 & 83.4 & 198 & 16.6 & \\
\hline No Chemotherapy & - & - & - & - & & - & - & - & - & & 18330 & 87.7 & 2561 & 12.3 & \\
\hline
\end{tabular}

$\mathrm{a}=\mathrm{t}$-test of mean differences

$\mathrm{b}=\mathrm{cmh}$ chi square test of general association

$\mathrm{c}=\mathrm{cmh}$ chi square test of location shift using table scores

$\mathrm{d}=\mathrm{cmh}$ chi square test of location shift using modified ridit scores

e $=$ Pearson's chi square test of association

${ }^{*} p<0.05 ;{ }^{* *} p<0.01 ;{ }^{* * *} p<0.001$

$\mathrm{BCS}=$ Breast-Conserving Surgery 
Table 3

Hazard of Death 5 Years After Diagnosis of Invasive Non-Metastatic Breast Cancer in Elderly Women

All Stage I, II, II

Stage I, II/Local Treatment

Stage I, II, III/GCC

SEER-Medicare 2003-2009

SEER-Medicare 2003-2009

SEER-Medicare 2004-2009

Dead vs. Alive

Dead vs. Alive

Dead vs. Alive

All

2003

2004

2005

AOR $\quad 95 \% \mathrm{Cl}$ Sig.

AOR $\quad 95 \% \mathrm{Cl}$ Sig. AOR

$95 \% \mathrm{Cl}$

Sig.

2006

1.00

0.94

$-$

1.00

0.88

$[0.88,1.01]$

0.95

$[0.82,0.94]$

$* * *$

0.88

$[0.88,1.03]$

$[0.81,0.95]$

0.82

$[0.75,0.89]$

$*$

0.77

$[0.71,0.84]$

$0.81 \quad[0.75,0.87]$

0.78

$[0.71,0.86]$

0.77

$[0.74,0.86]$

0.69

$[0.62,0.78]$

Age \& Health

Age at Diagnosis

$66-69$
$70-74$
$75-79$
$\geq 80$

1.00

\subsection{4}

1.86

4.01

$[0.71,0.85]$

Age \& Health

Comorbidity Score

0
1
$\geq 2$
PCP Visits
Low
High
Stage at Diagnosis
I
II
III

\subsection{0}

1.44

2.17

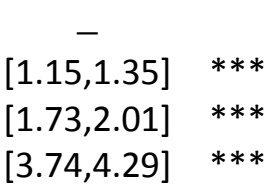

1.00

\subsection{1}

1.90

3.76

$[1.10,1.34]$

$[1.73,2.09]$

$[3.45,4.10]$

1.00

0.90

$[0.81,1.01]$

$* * *$

$0.92[0.83,1.03]$

$0.92[0.83,1.03]$

0.87

$[0.77,0.98]$

0.82

$[0.71,0.95]$

\subsection{0}

1.14

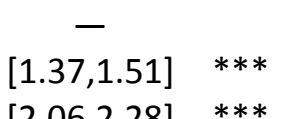

1.00

\subsection{9}

$-$

2.39

$[1.41,1.58]$

$[2.25,2.55]$

\subsection{0}

1.15

$[1.09,1.21]$

$* * *$

1.00

1.17

1.73

$[1.04,1.33] *$

$[1.54,1.95]$

$2.85 \quad[2.54,3.20]$

Clinical Prognostic Factors

\section{ER Status}

Positive

Negative

Borderline/Unknown

PR Status

Positive

Negative

Borderline/Unknown

Tumor Grade

Well Differentiated

Moderately Differentiated

Poorly Differentiated

Undifferentiated/Unknown
1.00

1.82

3.95

$-$

[1.74,1.91]

$[3.74,4.18]$

\begin{abstract}
1.00
\end{abstract}
1.66

$[1.57,1.74]$

$* * *$

1.00

1.00

$1.00 \quad[0.86,1.16]$

$1.81 \quad[1.48,2.21]$

\subsection{0}

1.36

1.22

$-$

$[1.27,1.45]$

1.00

\subsection{3}

1.08

$[1.23,1.45]$

1.00

1.48

0.72

$[1.32,1.65]$

$[0.87,1.35]$

\subsection{0}

1.00

1.19

1.09

-

$* * *$

1.17

1.04

$[1.09,1.26]$

$[0.84,1.29]$

1.00

1.13

0.88

$[1.03,1.25]$

$[0.59,1.31]$

\begin{tabular}{lcccccccc}
1.00 & - & & 1.00 & - & & 1.00 & - & \\
1.14 & {$[1.07,1.21]$} & $* * *$ & 1.15 & {$[1.08,1.24]$} & $* * *$ & 1.11 & {$[1.00,1.23]$} & \\
1.54 & {$[1.44,1.65]$} & $* * *$ & 1.49 & {$[1.38,1.61]$} & $* * *$ & 1.49 & {$[1.33,1.67]$} & $* * *$ \\
1.38 & {$[1.27,1.50]$} & $* * *$ & 1.09 & {$[0.98,1.22]$} & & 1.15 & {$[0.98,1.36]$} & \\
\hline
\end{tabular}

${ }^{*} \mathrm{p}<0.05 ;{ }^{* *} \mathrm{p}<0.01 ;{ }^{* * *} \mathrm{p}<0.001$

$\mathrm{BCS}=$ Breast-Conserving Surgery 
Table 3

Hazard of Death 5 Years After Diagnosis of Invasive Non-Metastatic Breast Cancer in Elderly Women

All Stage I, II, II

Stage I, II/Local Treatment

Stage I, II, III/GCC

SEER-Medicare 2003-2009

SEER-Medicare 2003-2009

SEER-Medicare 2004-2009

Dead vs. Alive

Dead vs. Alive

Dead vs. Alive

All AOR $\quad 95 \% \mathrm{Cl}$ Sig.

AOR $\quad 95 \% \mathrm{Cl}$ Sig. AOR

$95 \% \mathrm{Cl}$

Sig.

\section{Tumor Size \\ $<1 \mathrm{~cm}$ \\ $<2 \mathrm{~cm}$ \\ $2-5 \mathrm{~cm}$ \\ $>5 \mathrm{~cm}$}

Lymph Nodes

Positive

Negative

Mammography Screening Centers

Low

High

Oncology Treatment Centers

Low

High

Type of Surgeon Seen

General Only

Oncology Only

Both

Race

White

Other

Education

$<15 \%$ college degree

$\geq 15 \%$ college degree

Annual Income

$\leq \$ 35,000$

$>\$ 35,000$

Metro Status

Non-metro

Metro

Loco-Regional Treatment

BCS+Radiation

Mastectomy

BCS Only

Guideline-Concordant Care

Yes

No

Lymph Nodes Tested

\begin{tabular}{l} 
Yes \\
No \\
\hline p $<0.05 ;{ }^{* *} p<0.01 ;{ }^{* * *} p<0.001$
\end{tabular}

$\mathrm{BCS}=$ Breast-Conserving Surgery

$-$

$-$
1.00

$1.04 \quad[0.95,1.13]$

1.00

$0.96[0.88,1.04]$

$$
-
$$

$-$

$-$

$-$

1.00

1.36

1.90

$[1.20,1.54]$

$[1.62,2.23]$

$-$

$-$

$-$

2.30

$[1.89,2.80]$

$\begin{array}{ll}- & 1.00 \\ - & 0.68\end{array}$

$-$

Oncology Care Resources

\subsection{0}

0.78

0.68

\subsection{0}

$1.03[0.93,1.15]$

$[0.61,0.76]$

1.00

$1.05[0.93,1.17]$

1.00

$1.00 \quad[0.89,1.12]$

$0.93[0.84,1.04]$

1.00

1.00

$0.81 \quad[0.72,0.91]$

$0.72[0.68,0.76]$

0.74

0.78

$[0.62,0.87]$

$[0.71,0.85]$

Socio-Demographic Characteristics

$1.00-$

$1.00 \quad[0.93,1.06]$

\subsection{0}

$0.92[0.85,1.00]$

1.00

$0.99[0.89,1.10]$

$1.00-$

$0.92[0.88,0.97]$

$$
1.00
$$

0.91

1.00

0.94

$-$

$[0.89,1.00]$

$*$

1.00

$0.94 \quad[0.88,1.01]$

1.00

0.92

$[0.85,1.00]$

$1.03[0.96,1.09]$

1.00

$0.99 \quad[0.91,1.07]$

1.00

Treatment

$\begin{array}{llll}- & - & - & -\end{array}$

- $\quad-\quad$ -

$-$

1.81

1.94

$[1.70,1.93]$

$[1.82,2.06]$

\section{$-$}

$-$

$-$

$-\quad-$

$-$

$\begin{array}{lll}- & - & 1.00\end{array}$
$1.12[1.00,1.25]$

1.00

$-$

$-$

$1.09 \quad[0.98,1.21]$

\section{$* * *$ \\ $* * *$}

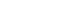

\section{$* * *$}

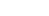


Table 3

Hazard of Death 5 Years After Diagnosis of Invasive Non-Metastatic Breast Cancer in Elderly Women

All Stage I, II, II

SEER-Medicare 2003-2009

Dead vs. Alive
Stage I, II/Local Treatment

SEER-Medicare 2003-2009

Dead vs. Alive

\begin{tabular}{|c|c|c|c|c|c|c|c|c|c|}
\hline All & AOR & $95 \% \mathrm{Cl}$ & Sig. & AOR & $95 \% \mathrm{Cl}$ & Sig. & AOR & $95 \% \mathrm{Cl}$ & Sig. \\
\hline \multicolumn{10}{|l|}{ ER Status Tested } \\
\hline Yes & - & - & - & - & - & - & 1.00 & - & \\
\hline No & - & - & - & - & - & - & 1.29 & {$[0.43,3.87]$} & \\
\hline \multicolumn{10}{|l|}{ PR Status Testede } \\
\hline Yes & - & - & - & - & - & - & 1.00 & - & \\
\hline No & - & - & - & - & - & - & 1.34 & {$[0.62,2.87]$} & \\
\hline \multicolumn{10}{|l|}{ Had any Surgery } \\
\hline Yes & - & - & - & - & - & - & 1.00 & - & \\
\hline No & - & - & - & - & - & - & 0.73 & {$[0.40,1.34]$} & \\
\hline \multicolumn{10}{|l|}{ Had BCS } \\
\hline Yes & - & - & - & - & - & - & 1.00 & - & \\
\hline No & - & - & - & - & - & - & 1.20 & {$[1.10,1.32]$} & $* * *$ \\
\hline \multicolumn{10}{|l|}{ Had Mastectomy } \\
\hline Yes & - & - & - & - & - & - & 1.00 & - & \\
\hline No & - & - & - & - & - & - & 1.10 & {$[0.99,1.23]$} & \\
\hline \multicolumn{10}{|l|}{ Had Radiation } \\
\hline Yes & - & - & - & - & - & - & 1.00 & - & \\
\hline No & - & - & - & - & - & - & 1.53 & {$[1.39,1.68]$} & $* * *$ \\
\hline \multicolumn{10}{|l|}{ Had Chemotherapy } \\
\hline Yes & - & - & - & - & - & - & 1.00 & - & \\
\hline No & - & - & - & - & - & - & 1.16 & {$[1.03,1.32]$} & $*$ \\
\hline \multicolumn{10}{|l|}{ Time to Chemotherapy } \\
\hline Appropriate & - & - & - & - & - & - & 1.00 & - & \\
\hline Not Appropriate & - & - & - & - & - & - & 1.22 & {$[1.02,1.46]$} & $*$ \\
\hline No Chemotherapy & - & - & - & - & - & - & - & - & \\
\hline
\end{tabular}

Stage I, II, III/GCC

SEER-Medicare 2004-2009

$* p<0.05 ; * * p<0.01 ; * * * p<0.001$

$\mathrm{BCS}=$ Breast-Conserving Surgery 

Appendix 1. Claims Codes Used for Identifying Types of Treatment

\begin{tabular}{|c|c|c|c|c|}
\hline Type of Treatment & ICD-9 Diagnostic & ICD-9 Procedure & HCPCS/CPT & Revenue Center \\
\hline Lymph Node Surgery & & $\begin{array}{l}40.3,40.41-40.42,40.50- \\
40.54,40.59,85.43 \\
85.48\end{array}$ & $\begin{array}{l}38500,38510,38520, \\
38525,38530,39542, \\
38720,38740,38745, \\
38792,78195,78800- \\
78801\end{array}$ & \\
\hline Lumpectomy & & $85.20-85.29$ & $\begin{array}{l}19120,19125-19126, \\
19160,19162,19301- \\
19302\end{array}$ & \\
\hline Mastectomy & & $85.33-85.36,85.40-85.48$ & $\begin{array}{l}19140,19180,19182, \\
19300,19303-19307, \\
19200,19220,19240, \\
19260,19271-19272\end{array}$ & \\
\hline Radiation Therapy & V58.0, V66.1, V67.1 & $92.20-92.39$ & $\begin{array}{l}\text { 77261-77799, G0256, } \\
\text { G0261, G0173-G0174, } \\
\text { G0243, G0251, G0338- } \\
\text { G03340 }\end{array}$ & 0330,0333 \\
\hline Chemotherapy & V58.1, V66.2, V67.2, & $99.25,99.28$ & $\begin{array}{l}\text { 96400-96599, C8953- } \\
\text { C8955, G0355-G0363, } \\
\text { G902-G9032, J0640, } \\
\text { J8510, J8520-J8521, } \\
\text { J8530-J8999, J9000- } \\
\text { J9999, Q0083-Q0085, } \\
\text { S9329-S9331, }\end{array}$ & $0331,0332,0335$ \\
\hline
\end{tabular}



Appendix 1. Guideline-Concordant Treatment Options by Tumor Size and Lymph Node Status

\begin{tabular}{|c|c|c|c|c|}
\hline Stage & \multicolumn{2}{|c|}{ Treatment Option A } & \multicolumn{2}{|c|}{ Treatment Option B } \\
\hline $\begin{array}{l}\text { Stage I: } \\
\qquad \mathrm{T} \leq 1 \mathrm{~cm}, \mathrm{~N}-\end{array}$ & $\begin{array}{ll}\text { - } & \text { BCS } \\
\text { - } & \text { RT } \\
\text { - } & \text { LNT } \\
\end{array}$ & - $\quad$ ERST \& PRST & $\begin{array}{ll}\text { - } & \text { Mastectomy } \\
\text { - } & \text { LNT } \\
\text { - } & \text { ERST \& PF }\end{array}$ & \\
\hline $\begin{array}{l}\text { Stage I: } \\
\qquad 1 \mathrm{~cm}<\mathrm{T} \leq 2 \mathrm{~cm}, \mathrm{~N}-\end{array}$ & $\begin{array}{ll}\text { - } & \text { BCS } \\
\text { - } & \text { RT } \\
\text { - } & \text { LNT }\end{array}$ & $\begin{array}{ll}- & \mathrm{CT} \\
- & \mathrm{CT}<120 \text { days } \\
\text { - } & \text { ERST \& PRST }\end{array}$ & $\begin{array}{ll}\text { - } & \text { Mastectomy } \\
\text { - } & \text { LNT } \\
\text { - } & \text { CT }\end{array}$ & $\begin{array}{ll}- & \mathrm{CT}<120 \text { days } \\
- & \text { ERST \& PRST }\end{array}$ \\
\hline $\begin{array}{l}\text { Stage IIA: } \\
\qquad 0 \mathrm{~cm} \leq \mathrm{T} \leq 2 \mathrm{~cm}, \mathrm{~N}+\end{array}$ & $\begin{array}{ll}- & \text { BCS } \\
\text { - } & \text { RT } \\
\text { - } & \text { LNT } \\
\end{array}$ & $\begin{array}{ll}- & \mathrm{CT} \\
- & \mathrm{CT}<120 \text { days } \\
- & \text { ERST \& PRST } \\
\end{array}$ & $\begin{array}{ll}- & \text { Mastectomy } \\
\text { - } & \text { LNT } \\
\text { - } & \text { CT } \\
\end{array}$ & $\begin{array}{ll}- & \mathrm{CT}<120 \text { days } \\
\text { - } & \text { ERST \& PRST }\end{array}$ \\
\hline $\begin{array}{l}\text { Stage IIA: } \\
\qquad 2 \mathrm{~cm}<\mathrm{T} \leq 5 \mathrm{~cm}, \mathrm{~N}-\end{array}$ & $\begin{array}{ll}\text { - } & \text { BCS } \\
\text { - } & \text { RT } \\
\text { - } & \text { LNT }\end{array}$ & $\begin{array}{ll}\text { - } & \mathrm{CT} \\
- & \mathrm{CT}<120 \text { days } \\
\text { - } & \text { ERST \& PRST }\end{array}$ & $\begin{array}{ll}\text { - } & \text { Mastectomy } \\
\text { - } & \text { LNT } \\
\text { - } & \text { CT }\end{array}$ & $\begin{array}{ll}\text { - } & \mathrm{CT}<120 \text { days } \\
\text { - } & \text { ERST \& PRST }\end{array}$ \\
\hline $\begin{array}{l}\text { Stage IIB: } \\
\qquad 2 \mathrm{~cm}<\mathrm{T} \leq 5 \mathrm{~cm}, \mathrm{~N}+\end{array}$ & $\begin{array}{ll}- & \text { BCS } \\
\text { - } & \text { RT } \\
\text { - } & \text { LNT } \\
\end{array}$ & $\begin{array}{ll}- & \mathrm{CT} \\
- & \mathrm{CT}<120 \text { days } \\
- & \text { ERST } \\
\end{array}$ & $\begin{array}{ll}\text { - } & \text { Mastectomy } \\
\text { - } & \text { LNT } \\
\text { - } & \text { CT } \\
\end{array}$ & $\begin{array}{ll}\text { - } & \mathrm{CT}<120 \text { days } \\
\text { - } & \text { ERST \& PRST }\end{array}$ \\
\hline $\begin{array}{l}\text { Stage IIB: } \\
\qquad \mathrm{T}>5 \mathrm{~cm}, \mathrm{~N}-\end{array}$ & $\begin{array}{ll}\text { - } & \text { Mastectomy } \\
\text { - } & \text { LNT } \\
\text { - } & \text { CT } \\
\end{array}$ & $\begin{array}{ll}\text { - } & \mathrm{CT}<120 \text { days } \\
\text { - } & \text { ERST \& PRST }\end{array}$ & & \\
\hline $\begin{array}{l}\text { Stage IIIA: } \\
\qquad 0 \mathrm{~cm} \leq \mathrm{T} \leq 5 \mathrm{~cm}, \mathrm{~N}+\end{array}$ & $\begin{array}{ll}\text { - } & \text { BCS } \\
\text { - } & \text { RT } \\
\text { - } & \text { LNT } \\
\end{array}$ & $\begin{array}{ll}\text { - } & \mathrm{CT} \\
\text { - } & \mathrm{CT}<120 \text { days } \\
\text { - } & \text { ERST } \\
\end{array}$ & $\begin{array}{ll}\text { - } & \text { Mastectomy } \\
\text { - } & \text { LNT } \\
\text { - } & \text { CT } \\
\end{array}$ & $\begin{array}{ll}\text { - } & \text { CT }<\text { 120days } \\
\text { - } & \text { ERST \& PRST } \\
\end{array}$ \\
\hline $\begin{array}{l}\text { Stage IIIA: } \\
\quad \mathrm{T}>5 \mathrm{~cm}, \mathrm{~N}+\end{array}$ & $\begin{array}{ll}\text { - } & \text { Mastectomy } \\
\text { - } & \text { LNT } \\
\text { - } & \mathrm{CT} \\
\end{array}$ & $\begin{array}{ll}- & \mathrm{CT}<120 \text { days } \\
- & \text { ERST \& PRST }\end{array}$ & & \\
\hline Stage IIIB \& IIIC & $\begin{array}{ll}\text { - } & \text { Mastectomy } \\
\text { - } & \text { LNT } \\
\text { - } & \text { CT } \\
\end{array}$ & $\begin{array}{ll} & \mathrm{CT}<120 \text { days } \\
- & \text { ERST \& PRST }\end{array}$ & & \\
\hline
\end{tabular}

$\mathrm{T}=$ tumor size; $\mathrm{N}=$ nodal status; $\mathrm{BCS}=$ breast-conserving surgery; $\mathrm{RT}=$ radiation therapy; $\mathrm{LNT}=$ lymph node testing; ERST = estrogen receptor status testing; PRST = progesterone receptor status testing; $\mathrm{CT}=$ chemotherapy; 
Figure 1. Kaplan Meier 5-Year Survival Curve of Elderly Women Diagnosed with Invasive NonMetastatic Breast Cancer, SEER-Medicare 2003-2009

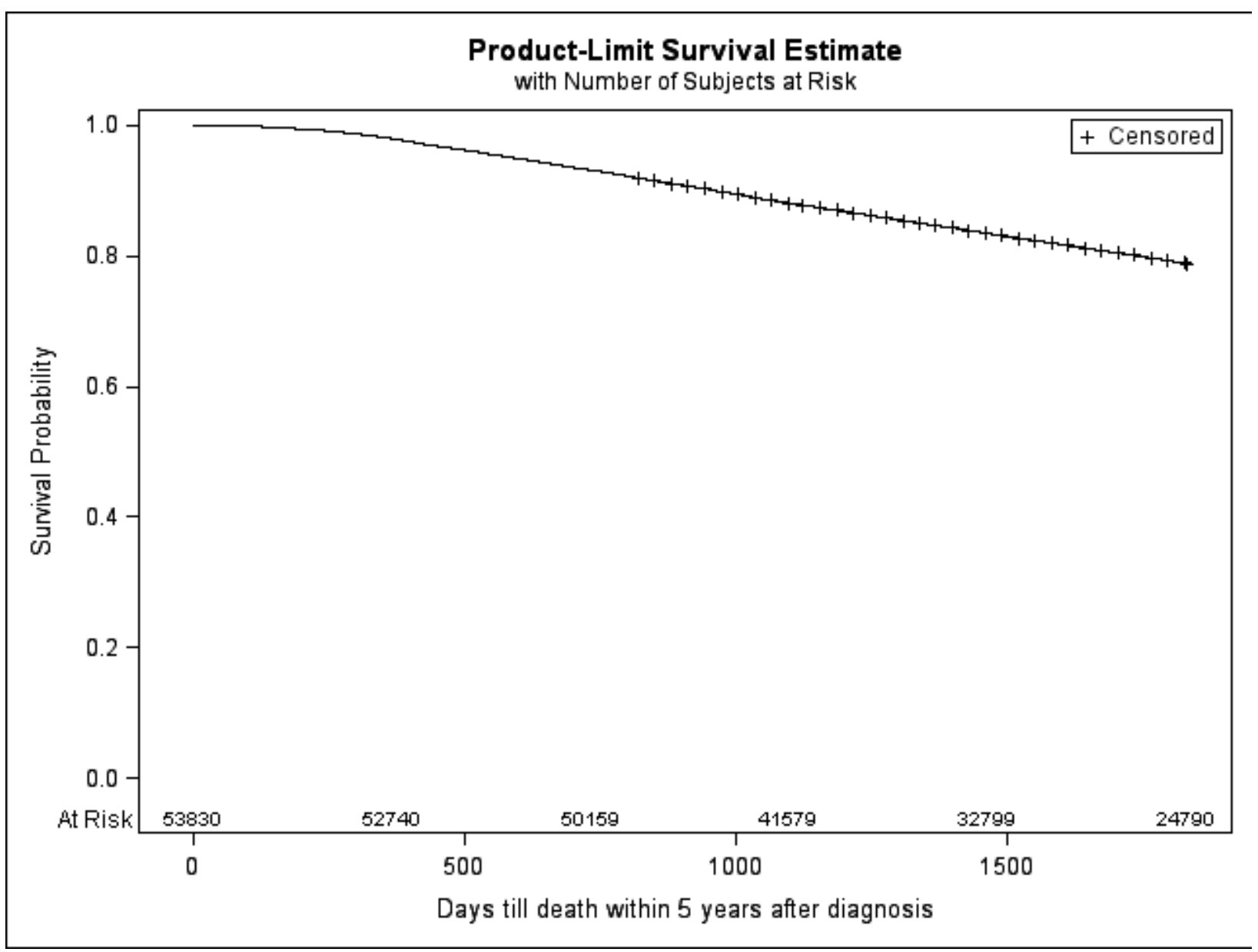


Figure 2. Kaplan Meier 5-Year Survival Curve of Elderly Women Diagnosed with Invasive NonMetastatic Breast Cancer By Stage, SEER-Medicare 2003-2009

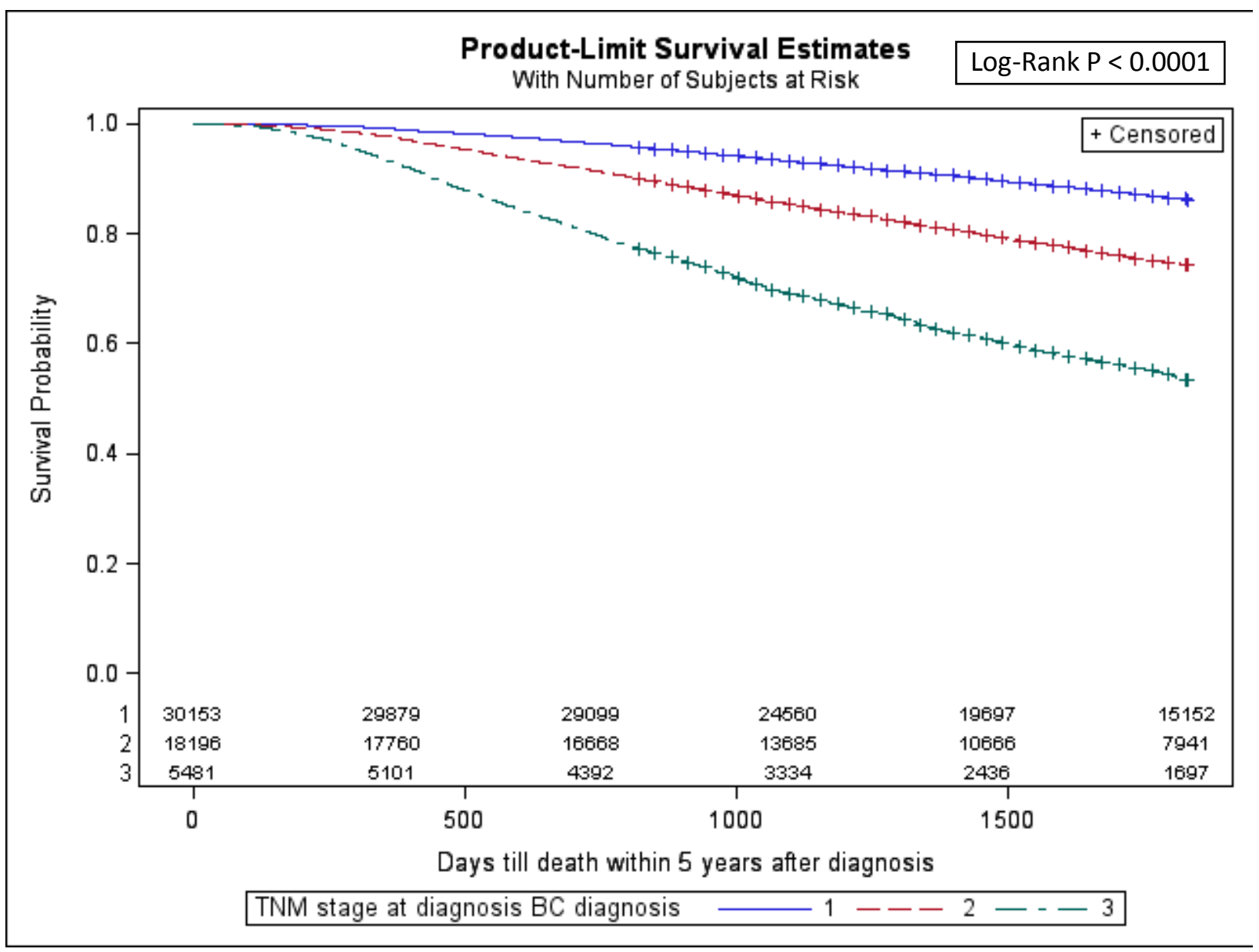


Figure 3. Kaplan Meier 5-Year Survival Curve of Elderly Women Diagnosed with Invasive EarlyStage Breast Cancer, SEER-Medicare 2003-2009

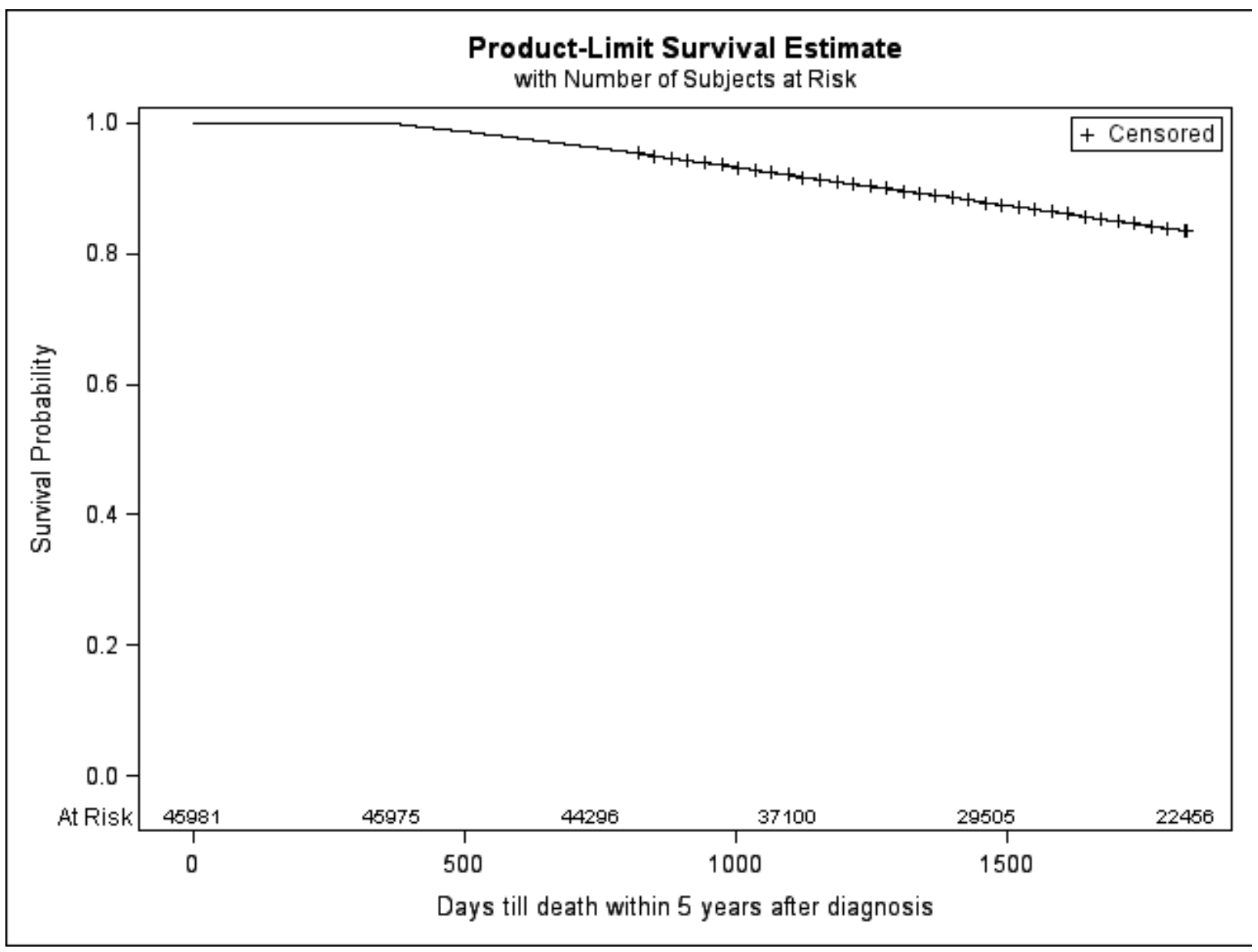


Figure 4. Kaplan Meier 5-Year Survival Curve of Elderly Women Diagnosed with Invasive EarlyStage Breast Cancer By Local Treatment, SEER-Medicare 2003-2009

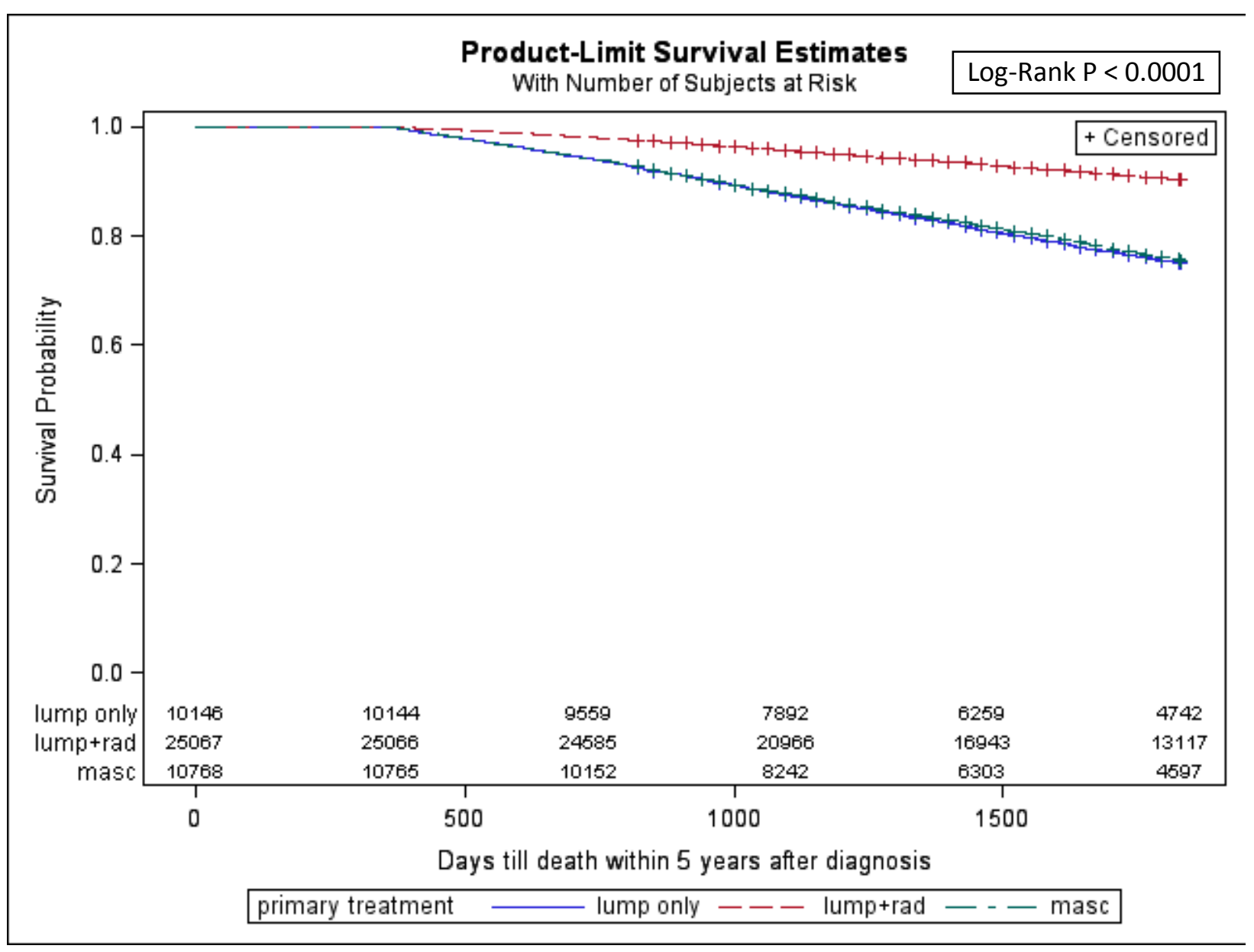


Figure 5. Kaplan Meier 5-Year Survival Curve of Elderly Women Diagnosed with Invasive NonMetastatic Breast Cancer, SEER-Medicare 2004-2009

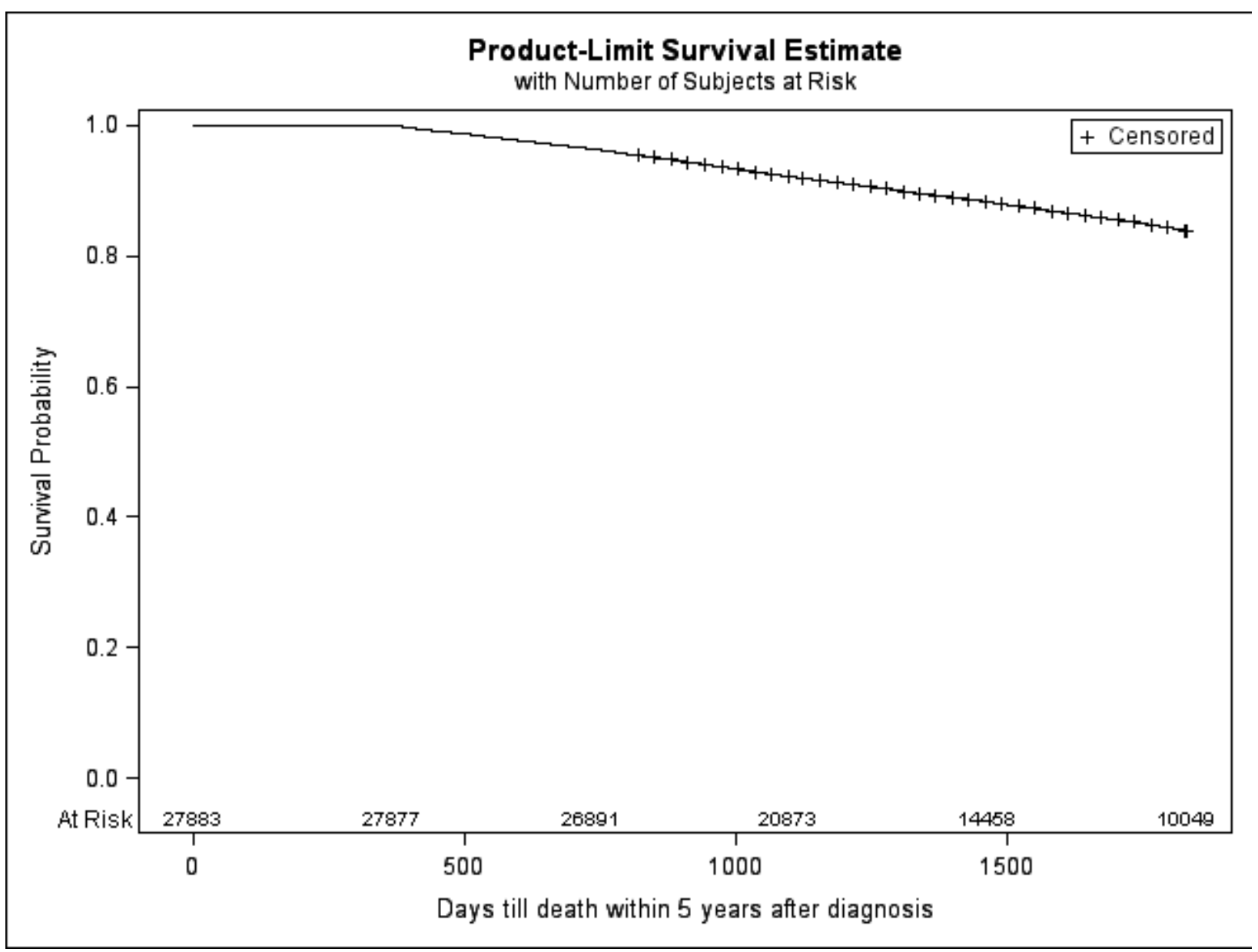


Figure 3. Kaplan Meier 5-Year Survival Curve of Elderly Women Diagnosed with Invasive NonMetastatic Breast Cancer By Guideline-Concordant Care, SEER-Medicare 2004-2009

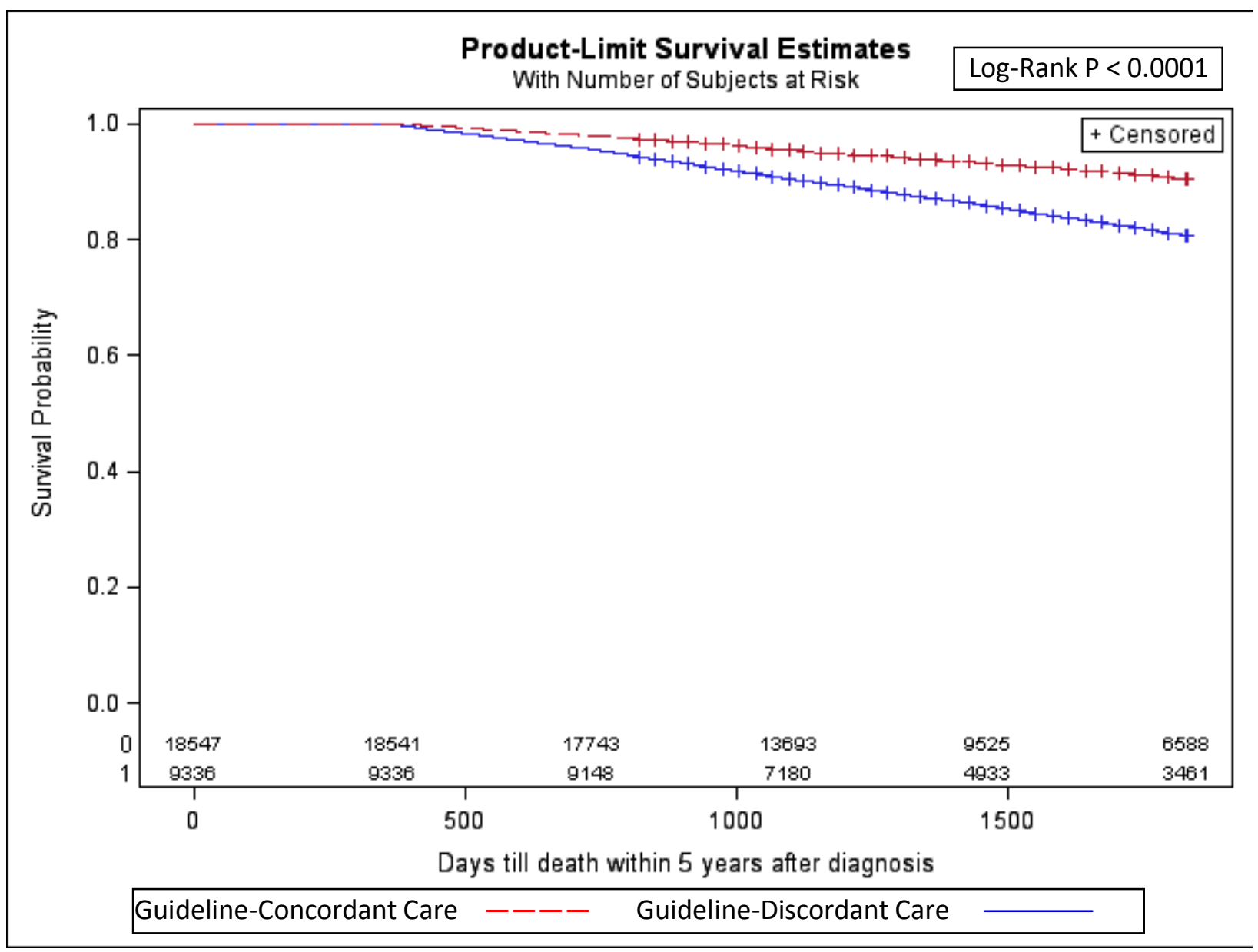


CHAPTER 5 


\section{CHAPTER 5}

\section{Study Review}

Epidemiology

Breast cancer is the second most commonly diagnosed type of cancer in the United States (US) and the first among women. ${ }^{1}$ It is estimated that in $2014,232,670$ incident diagnoses and 40,000 deaths will be attributed to breast cancer. The average age at diagnosis is 61 years. About $21 \%$ of breast cancer cases occur in women age $<50$ years, $22 \%$ among women age $50-59$ years, and $57 \%$ among women age $\geq 60$ years. ${ }^{2}$ While breast cancer is the most frequently diagnosed type of cancer among women in the US, it also has among the highest survival rates, relative to other types of cancer. Relative to women who have never been diagnosed with breast cancer, breast cancer survivors have $89 \%$ 5-year and $83 \%$ 10-year survival rates. ${ }^{3,4}$ Moreover, earlier stage diagnosis is associated with greater survival, such that women diagnosed at Stage I, II, and III have $100 \%, 93 \%$, and $72 \%$ 5-year survival rates, respectively. ${ }^{1}$ Decreasing mortality and increasing survival rates throughout the years are attributed to improvements in understanding of the disease and its treatment, alongside earlier detection due to increased routine breast cancer screening. 5

\section{Disparities in Treatment \& Survival}

Yet, despite these advancements, disparities in treatment patterns and survival outcomes persist. The most common disparities in treatment are the omission of recommended treatments and services or receiving non-preferential treatments. The most commonly omitted treatments are radiation therapy following breast-conserving surgery or mastectomy when indicated, chemotherapy, adjuvant hormone therapy, and lymph node sampling. While the course of breast cancer treatment is determined for each women by her tumor size, lymph node invasion, and 
hormone receptor status, women diagnosed at an early-stage often times have the option for breast-conserving surgery (i.e. lumpectomy) plus radiation therapy or mastectomy. Breastconserving surgery plus radiation therapy has been found to be an equally effective treatment as mastectomy for long-term survival of invasive early-stage breast cancer and is usually the preferred treatment choice due to breast preservation and functional performance. ${ }^{6-10}$ While about $60 \%$ or more women have BCS, one in four women who have BCS will undergo additional surgery, and about one in ten will eventually undergo mastectomy after treatment failure with BCS. ${ }^{11-15}$ Whether it is the initial choice of surgery or not, epidemiological studies have reported an increase in the use of mastectomy in recent years. ${ }^{12,13,16}$ Women who elect for mastectomy when BCS plus RT is an option tend to be younger, have a desire to avoid RT, fear recurrence, are diagnosed with later stage and more aggressive cancers, have cancers with clinical characteristics associated with poorer survival, are of lower income, insured by Medicaid or uninsured, reside in rural locations, have increased travel distances for RT, have a surgeon that is male surgeon, not trained in the U.S., and less recent training. ${ }^{12,13,16-21}$

In actuality, treatment disparities span across the entire course of breast cancer care, and lead to disparities in survival. Numerous factors have been associated with treatment and survival disparities, but several are particularly common among elderly women. Age and comorbidity are two inter-related factors that are strongly associated with treatment and survival outcomes. Due to an intolerance to toxic effects of radiation therapy and chemotherapy observed among some populations of older women, the National Comprehensive Cancer Network (NCCN) do not make recommendations for or against chemotherapy in women age $>70$ years, but suggest chemotherapy be given on a case-by-case basis, depending on the patient's health. ${ }^{22}$ Additionally, guidelines specify that radiation therapy following BCS may be omitted among 
women meeting the following requirements: age $\geq 70$ years, tumors $\leq 2 \mathrm{~cm}$, node negative, estrogen-receptor positive, and receiving adjuvant hormone therapy. ${ }^{23}$ Despite these recommendations, the decision to omit adjuvant therapies due to old age remains a topic of debate. What is more, study findings of treatment patterns among elderly women with breast cancer suggest that more women are not receiving radiation therapy and or chemotherapy than those who meet exemption criteria or may be too poor of health to undergo treatment. ${ }^{24-29}$ In turn, multiple studies have demonstrated poorer survival among elderly women who do not receive RT following BCS or chemotherapy when indicated. ${ }^{30-36}$

Additional factors associated with treatment and survival outcomes are sociodemographic characteristics, access to specialty oncology services, and clinical prognostic characteristics. Vulnerable socio-demographic characteristics such as being of non-white race, publicly insured or uninsured, being or residing in areas of low income, low education, residing in rural locations, and having greater driving distances to treatment centers are associated with breast cancer treatment disparities. ${ }^{37-42}$ Race is also directly associated with survival outcomes. Black women have markedly worse survival outcomes, as compared to white women, such that 5-year survival for white and black women is $90 \%$ and $79 \%$, respectively. ${ }^{3,43,44}$ Clinical characteristics associated with greater survival are earlier stage at diagnosis, smaller tumor size, less or no lymph node invasion, well differentiated tumor grade, and estrogen and progesterone positive tumors. ${ }^{1,45-47}$

\section{$\underline{\text { Statement of the Problem }}$}

To address gaps in the literature and build upon existing knowledge this series of studies focused upon the treatment patterns and survival of elderly women with breast cancer, a population with the highest incidence of the disease, but for whom many are undertreated due to 
age and declining health. Specifically, these studies sought to determine how age, health, clinical prognostic factors, oncology care resources, and socio-demographic characteristics are associated with these outcomes. The first study investigated the initial type of loco-regional treatment received for early-stage breast cancer. Most studies reporting on treatment patterns for early-stage breast cancer present rates that reflects the end result of multiple surgeries, such that a woman who first had BCS with or without RT, but eventually had a mastectomy, was classified as having a mastectomy and not BCS. This study captured, the initial treatment, with the intention of describing treatment preference and recommendation patterns among elderly women with early-stage breast cancer, as many women with this diagnosis have a choice of treatment. The second study sought to determine to what degree women with stage I, II, and III breast cancer receive GCC according to NCCN and ASCO treatment guidelines, by comprehensively comparing clinical characteristics and the care received by each women, including individual diagnostic tests and treatment, using a large, nationally representative dataset. In contrast, previous studies that have examined GCC in piecemeal fashion by studied narrowly defined groups of women, focusing on loco-regional or adjuvant therapies, or using smaller and older dataset. The third study will examine overall 5-year survival among the two previously described study populations and how each of those study outcomes (initial loco-regional treatment and receipt of individual tests, treatments, and GCC) and independent variables are associated with survival. Additionally, overall 5-year survival will be examined among a third, more broadly inclusive sample of women with stage I, II, III breast cancer, to gain a baseline understanding of overall survival of elderly women with invasive non-metastatic breast cancer.

In view of these findings, the goal of this dissertation was to 1) examine initial locoregional treatment patterns among elderly women with stage I and II breast cancer, 2) receipt of 
individual tests, treatments, and guideline-concordant care among elderly women with pathologically stage I, II, and III breast cancer, and 3) overall 5-year survival among a broadly inclusive group of elderly women with stage I, II, and III breast cancer and among the first two study samples using the linked SEER-Medicare dataset.

\section{Summary of Findings}

The first study sample consisted of 45,981elderly women diagnosed with stage I and II breast cancer in 2003 thru 2009. Of these women, about 55\% received breast-conserving surgery plus radiation therapy as their primary treatment, while $23 \%$ were treated with mastectomy and $22 \%$ had breast-conserving surgery only. About $63 \%$ were diagnosed at stage I, $23 \%, 26 \%, 23 \%$, and $29 \%$ were diagnosed at age $66-69,70-74,75-59$, and $\geq 80$ years, and $57 \%$ had a comorbidity score of zero. Receipt of mastectomy or breast-conserving surgery only, compared to breastconserving surgery plus radiation therapy, shared many of the same predictors with associations in the same direction. Compared to women who received breast-conserving surgery plus radiation therapy, those who were diagnosed at older age vs. age 66-69 years, had greater comorbidity vs. no comorbidity, diagnosed at stage II vs. stage I, of non-white race vs. white, lived in non-metro areas vs. metro, lived in areas of lower income vs. higher, lived in areas of lower education vs. higher, were treated by a general surgeon only vs. oncology or both specialties, and were more likely to receive mastectomy or breast-conserving surgery only. The second study sample was comprised of 27,883 women diagnosed with pathologically staged I, II, and III breast cancer in 2004 thru 2009. Among this sample, fewer than $34 \%$ of elderly women received guideline-concordant care as per NCCN and ASCO treatment guidelines. About 56\% of women were diagnosed at stage I, $34 \%$ at stage II, and $10 \%$ at stage III. Age at diagnosis was fairly evenly distributed, ranging from $24 \%$ - 27\% for each age group and $56 \%$ had a 
comorbidity score of zero. Ninety-five percent of women had their tumor estrogen-receptor, progesterone-receptor status, and $92 \%$ had their lymph nodes tested, but only $61 \%$ had radiation therapy and $25 \%$ had chemotherapy. About $75 \%$ of women had breast-conserving surgery and $42 \%$ of women initially or eventually had a mastectomy. Perhaps not coincidently, most of the same predictors of treatment for early-stage breast cancer that were not breast-conserving surgery plus radiation therapy, were also similarly associated with receipt of guidelineconcordant care among women with stage I, II, and III breast cancer. Older age, greater comorbidity, later stage at diagnosis, non-white race, residing in lower income areas, residing in lower education areas, and being treated by a general surgeon only were associated with a decreased likelihood of receiving guideline-concordant care. In fact, women age $\geq 70$ years old, were less than half as likely to receive guideline-concordant care, than women age 66-69 years at diagnosis. Women 70 years of age and older were $24 \%, 37 \%$, and $70 \%$ less likely to receive breast-conserving surgery, radiation therapy, or chemotherapy, respectively. The third study examined overall 5-year survival among the previously two described study samples and a broader sample ( $\mathrm{N}=53,830)$ of women diagnosed in 2003 thru 2009 with stage I, II, and III breast cancer. Across the three samples, 5-year survival rates ranged from $82 \%$ to $88 \%$, rates that are similar to those previously reported $89 \%$ 5-year survival rate for all women diagnosed with breast cancer in the US. Women who were diagnosed at age 66-69 years, stage I, had a comorbidity score $=0$, had positive estrogen and progesterone tumor receptors, and well differentiated tumors had the highest overall 5-year survival rates. Again, many of the same factors that were associated with less than optimal treatment in the first two studies, were associated with poorer survival. The risk of death within 5 years of diagnosis increased with each age category, such that women age $\geq 80$ years at diagnosis, were 2.8 to 4 times more likely 
to die. Similarly, women with a comorbidity score $\geq 2$ were more than twice as likely to die within 5 years of diagnosis. Additionally, the hazard of death within 5 years of diagnosis also increased with increasing stage at diagnosis, increasing tumor size, decreasing tumor grade, positive lymph node status, and negative estrogen or progesterone receptors statuses. The hazard of death was also increased for those who were treated by a general surgeon, mastectomy, breastconserving surgery only, did not have radiation therapy or chemotherapy, and did not have GCC.

\section{Discussion \& Conclusions}

Several common themes can be drawn from these study findings. First of all, many of the same reasons for health disparities that are prevalent among all women with breast cancer, or even the population at large, can also be observed among elderly women with breast cancer, who should in theory have uniform health insurance coverage. Whether it relates to receipt of preferential or guideline-concordant care, women who are characterized by vulnerable sociodemographic characteristics, such as non-white race, low income, low education, residing in rural areas, or areas with fewer oncology screening and treatment centers are more likely to experience health disparities in treatment. Elderly women with early-stage breast cancer of these characteristics more likely to be treated with breast-conserving surgery without radiation therapy or mastectomy when the option of breast-conserving surgery plus radiation therapy was an available option. What is more, vulnerable demographics of elderly women with all stages of invasive non-metastatic breast cancer were less likely to receive guideline-concordant care. Consequently, and perhaps for additional reasons, poorer overall 5-year survival rates were also observed among these same at risk populations. Taken together, these findings suggests that some women are better equipped to access care, regardless of the oncology service centers in their area. One cannot be certain why disparities persist among a population of women in the 
presence of equivalent health care coverage. Then again, women of white race, higher education, higher income, residing in metro areas or closer to oncology services may have more trust in health care providers, may be more comfortable navigating the health care system, may be more knowledgeable about breast cancer risk, treatment options, and prognosis, and may generally be more empowered to pursue and overcome treatment obstacles. These attributes that may lend themselves to increased mammography screening, thus earlier stage at diagnosis, and more aggressive and informed health care seeking behaviors. However, it is worth noting that vulnerable socio-demographic characteristics have additional consequences for overall breast cancer survival. Race, particularly black race, has been linked to poorer breast cancer survival due to aggressive types of breast cancer that are more frequently diagnosed among this group of women for biological reasons. Aside from biology, women who have lived a lifetime with limited access to health care or with riskier environmental and behavioral exposures may have poorer health and resiliency in old age that result in an increased risk of breast cancer mortality.

Another common theme that can be discerned from these study findings, is that elderly women experience unique breast cancer disparities because of their age. This finding is somewhat alarming because this is the demographic for which incidence of breast cancer is the greatest, but on the other hand, the under treatment and poor survival experienced by elderly women is somewhat intuitive given the health and functional declines associated with increasing age. The reason these findings can be both alarming and intuitive is because it is to be expected for women who are in too poor of health or functional status to be omitted from taxing treatments such as radiation therapy or chemotherapy, especially when their life-expectancy is relatively short. In fact, this is why treatment guidelines make such allowances or advise discretion in the use of such treatments for women older than 70 years of age. However, with 
that said, when only $34 \%$ of women over the age of 65 years receive guideline-concordant care or a little over half are treated with breast-conserving surgery plus radiation therapy, but almost a quarter receive breast-conserving surgery without radiation therapy, it seems probable that a greater number of women are not receiving the standard of care than there are of those who are actually in too poor of health to endure it. This seems to be a likely conclusion when one observes that more than two-thirds of the women with no comorbidity did not receive guidelineconcordant care. It is not clear why some women would not receive recommended treatments when they are healthy enough to do so. Possible reasons for under treatment may be that older women are more likely to refuse extensive treatments than younger women, it is too burdensome to arrange travel for repeated treatments, their families or caregivers do not believe it is in their best interest to undergo some treatments, or their oncologist may believe the benefits of such treatments do not outweigh their associated harms. Regardless of the reason for under treatment, the survival of women who could have benefited from aggressive treatment will be compromised. In addition to patterns of under treatment, the increasing obesity epidemic in the US and its associated chronic comorbid conditions further compromise the overall survival of elderly women with breast cancer.

\section{Future Research \& Direction}

Even though breast cancer treatment and survival have improved markedly over the past twenty-some years and increased mammography screening has increased rates of early stage diagnosis, disparities in treatment and survival still exist, with unique disparities affecting the

population with the highest incidence of breast cancer. While there is no logical reason for stage at diagnosis disparities to exist in the age of routine screening among a group of women with little out-of-pocket costs for the service, and yet they do exist and have negative impact on survival. Whether a later stage at diagnosis is attributed to declines in screening that are 
commonly observed among elderly women or for other reasons, health care providers and public awareness campaigns should emphasize mammography screening persistence among elderly women. Current screening guidelines put forth by the United States Preventive Services Task Force do not make recommendations for or against mammography screening after the age of 75 years. However, this should not be taken as an excuse to discontinue screening among older women. Similarly, treatment guidelines make exceptions for the omission of radiation therapy and chemotherapy among women older than 70 years, but it appears that more women than those who would meet this exemption do not receive these and other treatments when they would otherwise be indicated. Retrospective and secondary-database studies cannot definitively determine why these disparities exist. Prospective studies that can capture physician and patient perspective, in additional to clinical measures, are necessary to better understand the nature of this disparity in order to address it. Patient education and navigation programs available at the time of diagnosis could be helpful to further address treatment disparities among various vulnerable socio-demographic populations. These programs have shown to be beneficial for patients who are less knowledgeable about the disease and its treatment options, as well as, continuity of care and treatment completion. Beyond patient age and access to care, the other major factor associated with breast cancer disparities among the elderly is high rates of comorbidity. Yet, this pervasive problem that effects much of the US population may be harder to address. While the elderly may experience a wide range of health problems, many can be linked to obesity and unhealthy lifestyles. This health crisis has been the focus of many researchers, health care providers, government agencies, and other special interest groups. Although it is common knowledge that the way to preventing this problem is by being physically active and eating healthy foods, a mechanism for increasing these behaviors has yet to be 
identified. There will be no quick or easy solution to this problem, but efforts should continue to raise awareness and motive change to improve health, longevity, and resiliency for the population as a whole, and for elderly women with breast cancer.

Aside from the issues addressed by the current study, future studies of breast cancer outcomes among elderly women could investigate whether the improvement in targeted and lowdose radiation therapies increase radiation therapy rates among elderly women, the uptake of sentinel lymph node biopsy vs. axillary lymph node dissection across different populations of women, factors associated with repeated lumpectomy and treatment failure resulting in mastectomy, and factors associated with being treated by a general surgeon vs. an oncology surgeon. 


\section{References}

1. Howlader N, Noone A, Krapcho M, Garshell J, Miller D, Altekruse S, et al. (eds). SEER Cancer Statistics Review, 1975-2011, National Cancer Institute. Bethesda, MD, http://seer.cancer.gov/csr/1975_2011/, based on November 2013 SEER data submission, posted to the SEER web site, April 2014. Last accessed Mar 24, 2015.

2. DeSantis C, Lin C, Mariotto A, et al. Cancer treatment and survivorship statistics, 2014. CA Cancer J Clin. 2014; 64(4):252-271.

3. American Cancer Society. Cancer Facts \& Figures 2015. Atlanta: American Cancer Society; 2015. Available at: http://www.cancer.org/acs/groups/content/@editorial/documents/document/acspc044552.pdf. Last accessed on Jan 19, 2015.

4. American Cancer Society. Breast Cancer Facts \& Figures 2013-2014. Atlanta: American Cancer Society, Inc. 2013. Available at: http://www.cancer.org/acs/groups/content/@ research/documents/document/acspc042725.pdf. Last accessed on Jan 19, 2015.

5. Berry D, Cronin K, Plevritis S, Fryback D, Clarke L, Zelen M, et al. Effect of screening and adjuvant therapy on mortality from breast cancer. N Engl J Med. 2005; 353(17): 17841792.

6. Fisher B, Redmond C, Poisson R, Margolese R, Wolmark N, Wickerham L., et al. Eight-year results of a randomized clinical trial comparing total mastectomy and lumpectomy with or without irradiation in the treatment of breast cancer. N Engl J Med. 1989; 320(13): $822-828$.

7. Fisher B, Anderson S, Bryant J, Margolese R, Deutsch M, Fisher E, et al. Twenty-year follow-up trial comparing total mastectomy, lumpectomy, and lumpectomy plus irradiation for the treatment of invasive breast cancer. N Engl J Med. 2002; 347(16): $1233-241$.

8. Veronesi U, Cascinelli N, Mariani L, Greco M, Saccozzi R, Luini A, et al. Twenty-year follow-up of a randomize study comparing breast-conserving surgery with radical mastectomy for early breast cancer. N Engl J Med. 2002; 347(16): 1227-1232.

9. Litière S, Werutsky G, Fentiman I, Rutgers E, Christiaens M, Limbergen E, et al. Breast conserving therapy versus mastectomy for stage I-II breast cancer: 20 year follow-up of the EORTC 10801 phase 3 randomised trial. Lancet Oncol. 2012; 13(4): 412-19.

10. Simone N, Dan T, Shih J, Smith S, Sciuto L, Lita E, et al. Twenty-five year results of the national cancer institute randomized breast conservation trial. Breast Cancer Res Treat. 2012; 132(1): 197-203. 
11. American Cancer Society. Cancer Treatment and Survivorship Facts \& Figures 2014-2015. Atlanta: American Cancer Society; 2014.

12. Dragun A, Huang B, Tucker T, Spanos W. Increasing mastectomy rates among all age groups for early stage breast cancer: a 10-year study of surgical choice. Breast J. 2012; 18(4): 318-325.

13. Mahmood U, Hanlon A, Koshy M, Buras R, Chumsri S, Tkaczuk K, et al. Increasing national mastectomy rates for the treatment of early stage breast cancer. Ann Surg Oncol. 2013; 20(5): 1436-1443.

14. Wilke L, Czechura T, Wang C, et al. Repeat surgery after breast conservation for the treatment of stage 0 to II breast carcinoma: a report from the National Cancer Data Base, 2004-2010. JAMA Surg. 2014; 149(12): 1296-1305.

15. McCahill L, Single R, Aiello Bowles E, et al. Variability in reexcision following breast conservation surgery. JAMA. 2012; 307(5): 467-475.

16. McGuire K, Santillan A, Kaur P, Meade T, Parbhoo J, Mathias M, et al. Are mastectomies on the rise? A 13-year trend analysis of the selection of mastectomy versus breast conservation therapy in 5865 patients. Ann Surg Oncol. 2009; 16(10): 2682-2690.

17. Boscoe F, Johnson C, Henry K, Goldberg D, Shahabi K, Elkin E, et al. Geographic proximity to treatment for early stage breast cancer and likelihood of mastectomy. Breast. 2011; 20(4): 324-328.

18. Goyal S, Chandwani S, Haffty B, Demissie K. Effect of travel distance and time to radiotherapy on likelihood of receiving mastectomy. Ann Surg Oncol. 2014; Sept 23: Epub ahead of print.

19. Bradley C, Given C, Baser O, Gardiner J. Influence of surgical and treatment choices on the cost of breast cancer care. Eur J Health Econ. 2003; 4(2): 96-101.

20. Jacobs L, Kelley K, Rosson G, Detrani M, Chang D. Disparities in urban and rural mastectomy populations : the effects of patient- and county-level factors on likelihood of receipt of mastectomy. Ann Surg Oncol. 2008; 15(10): 2644-2652.

21. Schroen A, Brenin D, Kelly M, Knaus W, Slingluff C. Impact of patient distance to radiation therapy on mastectomy use in early-stage breast cancer patients. J Clin Oncol. 2005; 23(28): 7074-7080.

22. Carlson R, Allred D, Anderson B, Burstein H, Carter W, Edge S, et al. Breast cancer. Clinical practice guidelines in oncology. J Natl Compr Canc Netw. 2009; 7(2):122-192. 
23. Hughes K, Schnaper L, Berry D, Cirrincione C, McCormick B, Shank B, et al. Lumpectomy plus tamoxifen with or without irradiation in women 70 years of age or older with early breast cancer. N Engl J Med. 2004; 351(10): 971-977.

24. Tuttle T, Jarosek S, Habermann E, Yee D, Yuan J, Virnig B. Omission of radiation therapy after breast-conserving surgery in the United States. A Population-Based Analysis of Clinicopathologic Factors. Cancer. 2012; 118(8): 2004-2013.

25. Luu C, Goldstein L, Goldner B, Schoellhammer HF, Chen SL. Trends in radiotherapy after breast-conserving surgery in elderly patients with early-stage breast cancer. Ann Surg Oncol. 2013; 20(10): 3266-3273.

26. Nagar H, Yan W, Christos P, Chao KS, Nori D, Ravi A. Older patients with early-stage breast cancer: adjuvant radiation therapy and predictive factors for cancer-related death. Am J Clin Oncol. 2014; Oct. 20 Epub ahead of print.

27. McCormick B, Ottesen R, Hughes M, Javid S, Khan S, Mortimer J, et al. Impact of Guideline Changes on Use or Omission of Radiation in the Elderly with Early Breast Cancer: Practice Patterns at National Comprehensive Cancer Network Institutions. J Am Coll Surg. 2014; 219(4):796-802.

28. Mackey R, Chandru Kowdley G. Treatment practices and outcomes of elderly women with breast cancer in a community hospital. Am Surg. 2014; 80(7): 714-719.

29. Wheeler S, Carpenter W, Peppercorn J, Schenck A, Weinberger M, Biddle A. Predictors of timing of adjuvant chemotherapy in older women with hormone receptor-negative, stages II-III breast cancer. Breast Cancer Res Treat. 2012; 131(1): 207-216.

30. Shen X, Anne P, Keith S, Wojcieszynski A, Mishra M, Bar-Ad V, et al. Radiation therapy use and outcomes among older women with ER-positive and ER-negative stage I breast cancer. Am J Clin Oncol. 2014; 37(3): 241-247.

31. Smith B, Haffty B, Buchholz T, Smith G, Galusha D, Bekelman J, et al. Effectiveness of Radiation Therapy in Older Women With Ductal Carcinoma In Situ. J Natl Cancer Inst. 2006; 98(18): 1302-1310.

32. Truong P, Bernstein V, Lesperance M, Speers C, Olivotto I. Radiotherapy omission after breast-conserving surgery is associated with reduced breast cancer-specific survival in elderly women with breast cancer. Am J Surg. 2006; 191(6): 749-755.

33. Sun S, Hollenbeak C, Leung A. Deviation from the Standard of Care for Early Breast Cancer in the Elderly: What are the Consequences? Ann Surg Oncol. 2014; Dec 17: DOI 10.1245/s10434-014-4290-5 [Epub ahead of print]. 
34. Muss H, Berry D, Cirrincione C, Theodoulou M, Mauer A, Kornblith A, et al. Adjuvant Chemotherapy in Older Women with Early-Stage Breast Cancer. N Engl J Med 2009; 360(20): 2055-2065.

35. Giordano S, Duan Z, Kuo Y, Hortobagyi G, Goodwin J. Use and Outcomes of Adjuvant Chemotherapy in Older Women With Breast Cancer. J Clin Oncol. 2006; 24(18): 27502756.

36. Elkin E, Hurria A, Mitra N, Schrag D, Panageas K. Adjuvant Chemotherapy and Survival in Older Women With Hormone Receptor-Negative Breast Cancer: Assessing Outcome in a Population-Based, Observational Cohort. J Clin Oncol. 2006; 24(18): 2757-2764.

37. Anderson R, Morris C, Kimmick G, Trentham-Dietz A, Camacho F, Wu X, et al. Patterns of Locoregional Treatment for Nonmetastatic Breast Cancer by Patient and Health System Factors. Cancer. 2014; Nov 4. doi: 10.1002/cncr.29092. [Epub ahead of print].

38. Bhargava A \& Du X. Racial and socioeconomic disparities in adjuvant chemotherapy for older women with lymph node-positive, operable breast cancer. Cancer. 2009; 115(13): 2999-3008.

39. Smith G, Shih Y, Xu Y, Giordano S, Smith B, Perkins G, et al. Racial Disparities in the Use of Radiotherapy After Breast-Conserving Surgery. Cancer. 2010; 116(3): 734-741.

40. Jagsi R, Abrahamse P, Morrow M, Hawley S, Griggs J, Graff J, et al. Patterns and correlates of adjuvant radiotherapy receipt after lumpectomy and after mastectomy for breast cancer. J Clin Oncol. 2010; 28(14): 2396-2403.

41. Halpern M, Chen A, Marlow N, Ward E. Disparities in Receipt of Lymph Node Biopsy Among Early-Stage Female Breast Cancer Patients. Ann Surg Oncol. 2009; 16(3):562570 .

42. Dragun A, Huang B, Tucker T, Spanos W. Disparities in the application of adjuvant radiotherapy after breast-conserving surgery for early stage breast cancer: impact on overall survival. Cancer. 2011; 117(12): 2590-2598.

43. Iqbal J, Ginsburg O, Rochon P, Sun $\mathrm{P}^{1}$, Narod S. Differences in breast cancer stage at diagnosis and cancer-specific survival by race and ethnicity in the United States. JAMA. 2015; 313(2): 165-173.

44. Harper S, Lynch J, Meersman S, Breen N, Davis W, Reichman M. Trends in areasocioeconomic and race-ethnic disparities in breast cancer incidence, stage at diagnosis, screening, mortality, and survival among women ages 50 years and over (1987-2005). Cancer Epidemiol Biomarkers Prev. 2009; 18(1): 121-131. 
45. Rosenberg J, Chia Y, Plevritis S. The effect of age, race, tumor size, tumor grade, and disease stage on invasive ductal breast cancer survival in the U.S. SEER database. Breast Cancer Res Treat. 2005; 89(1): 47-54.

46. Fisher B, Redmond C, Fisher E, Caplan R. Relative worth of estrogen or progesterone receptor and pathologic characteristics of differentiation as indicators of prognosis in node negative breast cancer patients: findings from National Surgical Adjuvant Breast and Bowel Project Protocol B-06. J Clin Oncol. 1988; 6(7):1076-1087.

47. Hwang E, Lichtensztajn D, Gomez S, Fowble B, Clarke C. Survival after lumpectomy and mastectomy for early-stage invasive breast cancer: the effect of age and hormone receptor status. Cancer. 2013; 119(7): 1402-1411. 